\title{
CRPL-F 62
}

Vational Bureau of Standards

Library, N. W. Bidg

$\ln -264909$
Reterence book not to be taken from the Library.

\section{IONOSPHERIC DATA}

ISSUED

OCTOBER 1949 



\section{IONOSPHERIC DATA}

\section{CONTENTS}

Page

Symbols and Terminology; Conventions for

Determining Median Values ........... 2

Monthly Average and Median Values of Forld-Wide

Ionospheric Data

Ionospheric Data for Every Day and Hour at

Washington, D. C.

Ionosphere Disturbances ............ 7

American and Zürich Provisional Relative Sunspot

Numbers .................... 8

Solar Coronal Intensities Observed at Climax, Colorado . 9

Special Announcement (radio propagation notices broad-

cast on WWV )................. 9

Tables of Ionospheric Data ............ 10

Graphs of Ionospheric Data ........... 42

Index of Tables and Graphs of Ionospheric Data in CRPL-F62 


\section{SYMBOLS AND TERMINOLOGY; CONVENTIONS \\ FOR DETERMINING MEDIAN VALUES}

Beginning with data reported for January 1949, the symbols, terminology, and conventions for the determination of median values used in this report (CRPL-F series) conform as far as practicable to those adopted at the Fifth Meeting of the Internarional Radio Consultative Committee (C.C.I.R.) in Stockholm, 1948, and given in detail on pages 2 to 10 of the report CRPL 553 , "Ionospheric Data," issued January 1949.

For symbols and terminology used mith data prior to January 1949, see report IRPL-C61, "Report of International Radio Propagation Conference, "Tashington, 17 April to 5 May, 1944," previous issues of the F series, in particular, IRPL-F5, CRPL-F24, F33, F50, and report CRPL-7-1, "Preliminary Instructions for Obtaining and Reducing Manual Ionospheric Records."

Following the recommendations of the Nashington (1944) and Stockholm (1948) conferences, beginning ith data for January 1945, ruedian valuos are published wherever possible. Where averages are reported, they aro, at any hour, the average for all the days during the month for which numerical data exist.

In addition to the conventions for the determination of medians given in Appendix 5 of Document No. 293 E of the Stockholm conference, which are listed on pages 9 and 10 of CRPL-F53, the folloning conventions are used in determining the medians for hours when no measured values are given because of equipment limitations and ionospheric irregularities. Symbols used are those given on pages 2-9 of CRPL-P53 (Appendixes 1-4 of Document No. 293 E referred to above).

a. For all ionospheric characteristics:

Values missing because of $A, B, C, F, L, M, N, Q, R$, $S$, or $T$ (see terminology referred to abovo) are omitted from the median count.

b. For critical frequencies and virtual heights:

Values of foF2 (and foE near sunrise and sunset) missing because of $E$ are counted as equal to or less than the lower limit of the recorder. Values of $h^{1} F 2$ (and $h^{8} E$ near sunrise and sanset) missing for this reason are counted as equal to or greater than the median. Other characteristics missing because of $\mathrm{E}$ are omitted from the median count. See CRPL-F38, page 9.

Values missing because of D are counted as equal to or greater than the upper limit of the recorder. 
Values missing because of $G$ are counted:

1. For foF', as equal to or less than foFl.

2. For $1^{\prime} \mathrm{F} 2$, as equal to or greater than the median.

Values missing because of 7 are counted:

1. For foF2, as equal to or less than the median when it is apparent that $h^{\prime} F^{\prime} 2$ is unusually high; otherwise, values missing because of ir are omitted from the median count.

2. For $h^{1} F 2$, as equal to or greater than the median.

Values missing for any other reason are omitted from the median count.

c. For MUF factor (M-factors):

Values missing because of $G$ or $W$ are counted as equal to or less than the median.

Values missing for any other reason are omitted from the median count.

d. For sporadic E (Es):

Values of fEs missing because of $G$ (no Es reflections observed, the equipment functioning normally otherwise) are counted as equal to or less than the median foE, or equal to or less than the lower frequency count of the recorder.

Values of fEs missing for any other reason, and values of h'Es missing for any reason at all are omitted from the median count.

Beginning with data for November 1945, doubtful monthly median values for Lonospheric observations at Tashington, D. C., are indicated by parentheses, in accordance ith the practice already in use for doubtful hourly values. The following are the conventions used to determine whether or not a median value is doubtful:

1. If only four values or less are available, the data are considered insufficient and no median value is computed.

2. For the F2 layer, if only five to nine values are avallable, the median is considered doubtful. The $E$ and $F l$ layers are so regular in their characteristics that, as long as there are at least five values, the median is not considered doubtful.

3. For all layers, if more than half of the values used to compate the median are doubtful (either doubtful or interpolated), the median is considered doubtful.

The same conventions are used by the CRPL in computing the medians from tabulations of daily and hourly data for stations other than washington, beginning with the tables in IRPL-FI\&. 


\section{MONTHLY AVERAGE AND MEDIAN VALUES OF \\ WORLD - WIDE IONOSPHERIC DATA}

The ionospheric data given here in tables 1 to 51 and figures $I$ to 102. were assembled by the Central Radio Propagation Laboratory for analysis and correlation, incidental to CRPL prediction of radio propagation conditions. The data are median values unless otherwise indicated. The following are the sources of the data in this issue:

Austrelian Department of Supply and Shipping,

Bureau of Mineral Resources, Geology and Geophysics:

Watheroo, 田. Australia

British Department of Scientific and Industrial Research,

Radio Research Board:

Iindau/Harz, Germany

Radio Thave Research Leboratory, Central Broadcasting Administration:

Chungking, China

Lanchow, China

All India Radio (Government of India), New Delhi, India:

Bombay, India

Delhi, India

Madras, India

Tiruchirapaliz, India

Indian Council of Scientific and Industrial Research,

Radio Research Committee:

Calcutta, India

Electrical Communications Laboratory, Ministry of Communications:

Fukaura, Japan

Shibata, Japan

Tokyo, Japan

Wakkanai, Japan

Yamakswa, Japan

Ner Zealand Department of Scientific and Industrial Research: Christchurch, New Zealand

Norwegian Defense Research Establishment, Kjeller per Lillestrom, Norway: Oslo, Norway

South African Council for Scientiflc and Industrial Research:

Capetown, Union of $S$. APrica

Johannesburg, Union of $S$. Africa 
National Bureau of Standards (Central Radio Propagation Laboratory):

Baton Rouge, Louisiana (Louisiana State University)

Boston, Massachusetts (Harvard University)

Guam I.

Huancayo, Feru (Instituto Geofisico de Huancayo)

Maui, Hawaii

Palmyra $I$.

San Francisco, California (Stanford University)

San Juan, Puerto Rico (University of Puerto Rico)

Trinidad, Eritish Test Indies

Washington, D. C.

White Sands, New Mexico

The tables and graphs of ionospheric data are correct for the values reported to the CRPL, but, because of variations in practice in the interpretation of records and scaling and menner of reporting of values, may at times give an erroneous conception of typical ionospheric characteristics at the station. Some of the errors are due to:

a. Differences in scaling records when spread echoes are present.

b. Omission of values when foF 2 is less than or equal to foFl, leading to erroneously high values of monthly averages or median values.

c. Omission of values when critical frequencies are less than the lower frequency limit of the recorder, also leading to erroneously high values of monthly average or median values.

These effects were discussed on pages 6 and 7 of the previous F-series report IRPL-F5.

Ordinarily a blank space in the fEs column of a table is the result of the fact that a majority of the readings for the month are below the lower limit of the recorder or less than the corresponding values of $f \circ E$. Blank spaces at the beginning and end of columns of $h^{\prime} \mathrm{Fl}$, fOFl, $h^{\prime} E$, and foE are usually the result of diurnal variation in these characteristics. Complete absence of medians of h'Fl and foFl is usually the result of seasonal effects. 
The dashed-Iine prediction curves of the graphs of ionospheric data are obtained from the predicted zero-muf contour charts of the CRPL-D series publications. The following points are worthy of note:

a. Predictions for indiviaual stations used to construct the charts may be more accurate than the values read from the charts since some smoothing of the contours is necessary to allow for the loneitude effect within a zone. Thus, inasmuch as the predicted contours are for the center of each zone, part of the discrepancy between the predicted and observed values as given in the $F$ series may be caused by the fact that the station is not centrally located within the zone.

b. The final presentation of the predictions is dependent upon the latest available ionospheric and radio propagation data, as well as upon predicted sunspot number.

c. There is no indication on the graphs of the relative reliability of the data; it is necessary to consult the tables for such information.

The following predicted smoothed 12-month running-average zürich sunspot numbers were used in constructing the contour charts:

\begin{tabular}{lccccc} 
Month & \multicolumn{5}{c}{ Predicted Sunspot No. } \\
\cline { 2 - 5 } & 1949 & 1948 & 1947 & 1946 & 1945 \\
\hline December & & 114 & 126 & 85 & 38 \\
November & & 115 & 124 & 83 & 36 \\
October & & 116 & 119 & 81 & 23 \\
September & 115 & 117 & 121 & 79 & 22 \\
August & 111 & 123 & 122 & 77 & 20 \\
July & 108 & 125 & 116 & 73 & \\
June & 108 & 129 & 112 & 67 & \\
llay & 108 & 130 & 109 & 67 & \\
April & 109 & 133 & 107 & 62 & \\
llarch & 111 & 133 & 105 & 51 & \\
February & 113 & 133 & 90 & 46 & \\
January & 112 & 130 & 88 & 42 & \\
\hline
\end{tabular}

\section{IONOSPHERIC DATA FOR EVERY DAY AND HOUR} AT WASHINGTON, D. C.

The data given in tables 52 to 63 follow the scaling practices given in the report IRPL-C6I, "Report of International Radio Propagation Conference," pages 36 to 39, and the median values are determined by the conventions given above under "Symbols and Terminology; Conventions for Determining Median Values." Beginning with September 1949, the data are taken at a new location, Ft. Belvoir, Virginia. 
Table 64 presents ionosphere character figures for Washington, D. C., during September 1949, as determined by the criteria presented in the report IRPL-R5, "Criteria for Ionospheric Storminess," together with Cheltenham, Maryland, geomagnetic K-figures, which are usually covariant with them.

Table 65 lists for the stations whose locations are given the sudden ionosphere disturbances observed on the continuous field intensity recordings made at the Sterling Radio Propagation Laboratory during September 1949.

Table 66 Iists for the stations whose locations are given the sudden ionosphere disturbances observed at the Brentwood and Somerton, England, receiving stations of Cable and Wireless, Ltd., for various days in August and September 19,49 .

Table 67 lists for the stations whose locations are given the sudden ionosphere disturbances observed at the Platanos, Argentina, receiving station of the International Telephone and Telegraph Corporation for August 3, 1949.

Table 68 lists for the stations whose locations are given the sudden ionosphere disturbances observed at the Point Reyes, California, receiving station of RCA Communications, Inc., for several days in september and for October 2, 1949.

Table 69 lists for the stations whose locations are given the sudden ionosphere djsturbances observed at the Riverhead, New York, receiving station of RCA Cormunications, Inc., for various days in September and October 1949.

Table 70 gives provisional radio propagation quality figures for the North Atlantic and North Pacific areas, for 01 to 12 and 13 to $24 \mathrm{GCT}$, August 1949, compared with the CRPL daily radio disturbance warnings, which are primarily for the North Atlantic paths, the CRPL weekly radio propagation forecasts of probable disturbed periods, and the half-day Cheltenham, Maryland, geomagnetic K-figures.

The radio propagation quality figures are prepared from radio traffic and ionospheric data reported to the CRPL, in a manner basically the same as that described in IRPL-R3I, "North Atlantic Radio Propagation Disturbances, October 1943 through October 1945," issued February 1, 1946. The scale conversions for each report are revised for use with the data beginning January 19,48, and statistical welghting replaces what was, in effect, subjective weighting. Separate master distribution curves of the type described in IRPL-R31 were derived for the part of 1946 covered by each report; data received only since 1946 are compared with the master curve for the period of the available data. A report whose distribution is the same as the master is thereby converted linearly to the Q-figure scale. Each report is given a statistical weight which is the reciprocal of the departure from Iinearity. The half-dally radio propagation quality figure, beginning January 1948, is the weighted mean of the reports received for that period. 
These radio propagation quality figures give a consensus of opinion of actual radio propagation conditions as reported by the half day over the two general areas. It should be borne in mind, however, that though the quality may be disturbed according to the CRPL scale, the cause of the disturbance is not necessarily known. There are many variables that must be considered. In addition to ionospheric storminess itself as the cause, conditions may be reported as disturbed because of seasonal characteristics such as are particularly evident in the pronounced day and night contrast over North Pacific paths during the winter months, or because of improper frequency usage for the path and time of day in question. Insofar as possible, frequency usage is included in rating the reports. Where the actual frequency is not shown in the report to the CRPL, it has been assumed that the report is made on the use of optimum working frequencies for the path and time of day in question. Since there is a possibility that all the disturbance shown by the quality figures is not due to ionospheric storminess alone, care should be taken in using the quality figures in research correlations with solar, auroral, geomagnetic, or other data. Nevertheless, these quality figures do reflect a consensus of opinion of actual radio propagation conditions as found on any one half day in either of the two general areas.

\section{AMERICAN AND ZÜRICH PROVISIONAL RELATIVE SUNSPOT NUMBERS}

Table 71 presents the daily American relative sunspot number, $R_{\mathbb{A}}$, computed from observations communicated to CRPL by observers in America and abroad. Beginning with the observations for January 1948, a new method of reduction of observations is employed such that each observer is assigned a scale-determining "observatory coefficient," ultimately referred to zthich observations in a standard period, December 1944 to September 1945, and a statistical weight, the reciprocal of the variance of the observatory coefficient. The daily numbers listed in the table are the weighted means of all observations received for each day. Details of the procedure are given in the Publication of the Astronomical Society of the Pacific, issued February 1949, in an article entitled "Reduction of Sunspot-Number Observations." The American relative sunspot number computed in this way is designated $R_{A}$. It is noted that a number of observatories abroad, including the idureh. observatory, are included in $R_{A}$. The scale of $R_{A}$ was referred specifically to that of the Züich relative sunspot numbers in the standard comparison period; since that time, $R_{A}$ is influenced by the zurich observations only in that $\mathrm{Zl}$ rich proves to be a consistent observer and receives a high statistical weight. In addition, this table lists the daily provisional Zurich sunspot numbers, $R_{Z}$. 


\section{SOLAR CORONAL·INTENSITIES OBSERVED}

\section{AT CLIMAX, COLORADO}

In tables $72 \mathrm{a}$ and $72 \mathrm{~b}$ are listed the intensities of the green (5303A) line of the emission spectrum of the solar corona as observed during September 1949 by the High Altitude Observatory of Harvard University and the University of Colorado at Climax. Colorado, for east and west limbs, respectively, at 5-degree intervals of position angle north and south of the solar equator at the limb. Beginning January 11, 1949, the actual measurements are on solar rotation coordinates rather than astronomical coordinates; thus values of the correction $P$ given in previous coronal tables are omitted. The time of observation is given to the nearest tenth of a day, GCT. The tables of coronal observations in CRPL-F29 to F4I listed the data on astronomical coordinates; the present format on solar rotation coordinates is in conformity with the tables of CRPL-1-4, "Observations of the Solar Corona at CIimax, 1944-46."

Tables $73 \mathrm{a}$ and $73 \mathrm{~b}$ give similarly the intensities of the first red (6374A) coronal line; tables $74 \mathrm{a}$ and $74 \mathrm{~b}$ list the intensities of the second red (6704A) coronal line. The following symbols are used in tables 72,73 , and 74: a, observation of low weight; -, corona not visible; and $x$, position angle not included in plate estimates.

\section{SPECIAL ANNOUNCEMENT}

Effective November 1, 19,49, the detail of the radio propagation disturbance notices, broadcast on the National Bureau of Standards Radio Station WN, will be extended by the addition of a third category. Heretofore, two grades of propagation conditions have been recognized in the notices given at nineteen and forty-nine minutes past each hour. The letter "N" (in International Morse Code) repeated eight times has signified normal conditions, while the letter "W" has constituted a warning that disturbed conditions were present or expected within 12 hours. The third category, denoted by the letter "U", is being added to describe unstable conditions -- satisfactory performance for services employing high-power transmitting equipment operating on the recommended frequency, but poor reception on less well-equiped services. The propagation disturbance notices primarily refer to North Atlantic radio circuits. Details are included in NBS Letter Circular LC 886, available upon request. 


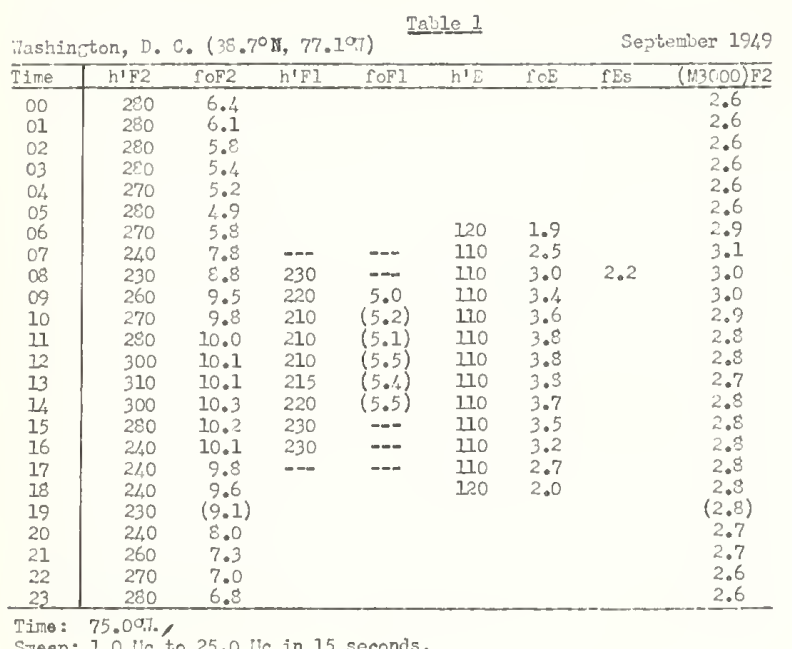

S7reep: 1.0 :lc to 25.0 lltc in 15 seconds.

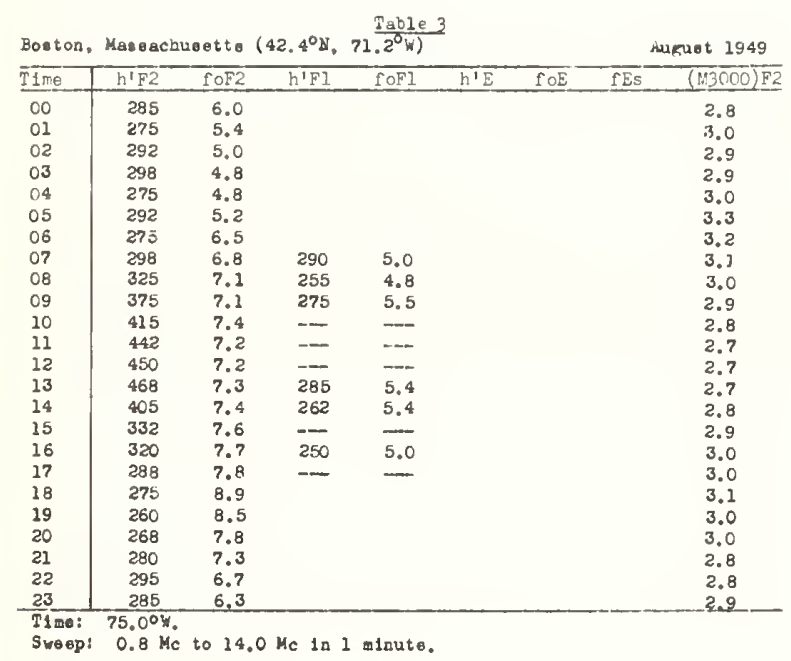

Tubla 5

\begin{tabular}{|c|c|c|c|c|c|c|c|c|}
\hline$\overline{1 m \theta}$ & $h^{1} F 2$ & foF2 & $h^{\prime} F I$ & foFl & $h^{\prime} E$ & foE & $f E S$ & $( \mathrm { M } 3 0 0 0 ) \longdiv { \mathrm { F } 2 }$ \\
\hline 00 & 300 & 5.5 & & & & & 2.4 & 2.5 \\
\hline 01 & 300 & 5.2 & & & & & 2.9 & 2.6 \\
\hline 02 & 290 & 5.2 & & & & & 2.9 & 2.6 \\
\hline 03 & 285 & 5.0 & & & & & 2.4 & 2.7 \\
\hline 04 & 280 & 4.8 & & & & & 2.6 & 2.6 \\
\hline 06 & 280 & 4.6 & & & & & 2.8 & 2.6 \\
\hline 06 & 260 & 5.8 & 260 & -- & 110 & $(2.0)$ & 3.9 & 2.8 \\
\hline 07 & 240 & 7.2 & 240 & 4.0 & 110 & 2.7 & 5.0 & 2.8 \\
\hline 08 & 295 & 8.0 & 230 & 4.5 & 110 & $(3.1)$ & 4,9 & 2.7 \\
\hline 09 & 336 & 8.4 & 220 & 5.0 & 110 & 3.4 & 5.0 & 2.6 \\
\hline 10 & 370 & 8.6 & 220 & 5.3 & 110 & 3.6 & 5.1 & 2.6 \\
\hline 11 & 370 & 9.5 & 220 & 5.3 & 110 & 3.8 & 4.7 & 2.6 \\
\hline 12 & 370 & 9.5 & 220 & 5.4 & 110 & 3.9 & 4.8 & 2.6 \\
\hline 13 & 360 & 9.4 & 220 & 5.4 & 110 & 3.9 & 4.6 & 2.6 \\
\hline 14 & 350 & 9.7 & 225 & 5.2 & 110 & 3.8 & 4.9 & 2.6 \\
\hline 15 & 340 & 9.3 & 230 & 5.1 & 110 & 3.6 & 4. 7 & 2.6 \\
\hline 16 & 320 & 9.3 & 240 & 4.9 & 110 & 3.4 & 4.5 & 2.7 \\
\hline 17 & 280 & 9.1 & 240 & 4.3 & 110 & 2.8 & 4.6 & 2.8 \\
\hline 18 & 260 & 8.6 & 245 & - & 110 & $(2.2)$ & 3.4 & 2.8 \\
\hline 19 & 250 & 7.8 & & & & & 2.8 & 2.9 \\
\hline 20 & 240 & 7.0 & & & & & 2.7 & 2.8 \\
\hline 21 & 260 & 6.4 & & & & & 2.6 & 2.7 \\
\hline 22 & 280 & 5,8 & & & & & 2.7 & 2.6 \\
\hline 23 & 310 & 5.4 & & & & & 2.3 & 2.5 \\
\hline
\end{tabular}

Treep: $0.8 \mathrm{Hc}$ to $14.0 \mathrm{Mc}$ in 2 minuter.

\begin{tabular}{|c|c|c|c|c|c|c|c|c|}
\hline \multicolumn{4}{|c|}{ Os10. No rway $\left(6 C .0^{\circ} \mathrm{N}, 11.0^{\circ} \mathrm{E}\right)$} & \multicolumn{3}{|c|}{ Trolo 2} & \multicolumn{2}{|c|}{ Auguet 1949} \\
\hline ime & $n 1 F 2$ & foF2 & $h^{\prime} F 1$ & fOFI & $h^{\prime} \bar{L}$ & $\mathrm{fOE}$ & fEs & $(13300 \overline{F 2}$ \\
\hline 00 & 290 & 4.8 & & & & & & \\
\hline 01 & 295 & 4.2 & & & & & 2.3 & \\
\hline 02 & 300 & 4.1 & & & & & 2.4 & \\
\hline 03 & 300 & 3.9 & & & & & 2.4 & \\
\hline 04 & 280 & 3.8 & -- & $\cdots$ & & & 2.7 & \\
\hline 05 & 250 & 4.7 & - & - & 140 & 1.8 & 2.4 & \\
\hline 06 & 262 & 5.4 & 240 & -- & 120 & 2.2 & 2.5 & \\
\hline 07 & 275 & 6.0 & 240 & 4.0 & 110 & 2.6 & 3.2 & \\
\hline 08 & 318 & 6.3 & 230 & 42 & 110 & 2.9 & 3.6 & \\
\hline 09 & 315 & 6.5 & 220 & 4.5 & 105 & 3.0 & 3.8 & \\
\hline 10 & 325 & 6.7 & 215 & 4.7 & 105 & 3.1 & 4.0 & \\
\hline 11 & 325 & 6.9 & 210 & 4.7 & 105 & 3.3 & 4.0 & \\
\hline 12 & 352 & 6.8 & 210 & 4.8 & 100 & 3.4 & 3.7 & \\
\hline 13 & 365 & 6.8 & 215 & 4.9 & 100 & 3.4 & 3.6 & \\
\hline 14 & 342 & 6.9 & 215 & 4.7 & 305 & 3.4 & 3.5 & \\
\hline 15 & 322 & 6.9 & 220 & 4.7 & 105 & 3.2 & & \\
\hline 16 & 310 & 7.0 & 230 & 4.5 & 105 & 3.1 & & \\
\hline 17 & 285 & 7.1 & 240 & - & 110 & 2.8 & 3.1 & \\
\hline 18 & 255 & 7.2 & 242 & - & 115 & 2.4 & 2.8 & \\
\hline 19 & 250 & 6.9 & 250 & $\rightarrow$ & 130 & 2.0 & 2.7 & \\
\hline 20 & 250 & 6.5 & & & - & $-\cdots$ & 2.0 & \\
\hline 21 & 250 & 6.5 & & & - - & -- & & \\
\hline 22 & 252 & 5.9 & & & & & & \\
\hline 23 & 270 & 5,3 & & & & & & \\
\hline
\end{tabular}

\begin{tabular}{|c|c|c|c|c|c|c|c|c|}
\hline \multicolumn{7}{|c|}{ San Francisco, Callfornis $\left(37.4^{\circ} \mathrm{N}, 122.2^{\circ} \mathrm{W}\right)$} & \multicolumn{2}{|c|}{ Augugt 1949} \\
\hline Time & $h^{\prime} F 2$ & fOF2 & $h^{\prime} F I$ & rof $]$ & $h^{\prime} E$ & foE & fES & $(\mathrm{M} 3000) \overline{\mathrm{F} 2}$ \\
\hline 00 & 310 & 5.2 & & & & & 2.8 & 2.7 \\
\hline 01 & 305 & 5.2 & & & & & 2.7 & 2.7 \\
\hline 02 & 300 & 5.1 & & & & & 2.2 & 2.7 \\
\hline 03 & 300 & 5.0 & & & & & 2.4 & 2.7 \\
\hline 04 & 300 & 4.8 & & & & & 2.4 & 2.7 \\
\hline 05 & 300 & 4.5 & & & & & 2.5 & 2.6 \\
\hline 06 & 270 & 5.4 & 255 & 2.5 & 120 & 2.2 & 2.8 & 2.9 \\
\hline $0 ?$ & 270 & 7.0 & 240 & 4.0 & 120 & 2.9 & 3.9 & 2.8 \\
\hline 08 & 310 & 7.5 & 230 & 4.6 & 120 & 3.4 & 3.3 & 2.8 \\
\hline 09 & 320 & 8.4 & 220 & 5.1 & 120 & $(3.7)$ & 4.2 & 2.7 \\
\hline 10 & 340 & 8.7 & 210 & 5.2 & 120 & $(3,8)$ & 3.6 & 2.7 \\
\hline 11 & 360 & 9.2 & 210 & 5.4 & 120 & 4.0 & & 2.7 \\
\hline 12 & 355 & 9.3 & 220 & 5.2 & 115 & $(4,0)$ & & 2.7 \\
\hline 13. & 350 & 9.4 & 230 & 5.3 & 110 & 4.0 & & 2.7 \\
\hline 14 & 340 & 9.2 & 235 & 5.2 & 110 & $(4,0)$ & & 2.8 \\
\hline 15 & 335 & 9.0 & 230 & 6.2 & 110 & 3.6 & 4.0 & 2.8 \\
\hline 16 & 315 & 8.8 & 240 & 4.8 & 110 & $(3.4)$ & 4.0 & 2.8 \\
\hline 17 & 290 & 8.4 & 260 & 4.4 & 120 & 3.2 & 3.8 & 2.9 \\
\hline 18 & 260 & 8.2 & $-\infty$ & $=$ & 120 & 2.4 & 3.6 & 2.9 \\
\hline 19 & 250 & 7.6 & & & & & 3.2 & 3.0 \\
\hline 20 & 250 & 7.0 & & & & & 2.8 & 2.9 \\
\hline 21 & 260 & 6.4 & & & & & 2.8 & 2.8 \\
\hline 22 & 280 & 5.6 & & & & & 2.8 & 2.8 \\
\hline 23 & 300 & 5.4 & & & & & 2.4 & 2.7 \\
\hline
\end{tabular}

Swoop: $1.3 \mathrm{Hc}$ tc $18.0 \mathrm{Mc}$ in 4 sinutoo.

Table 6

\begin{tabular}{|c|c|c|c|c|c|c|c|c|}
\hline$T 1 \pi \theta$ & $\bar{h} 1 F 2$ & foF2 & $h+F I$ & $f \circ F]$ & $h^{\prime} E$ & $f \circ E$ & $f E s$ & $(M 3000) \sqrt{F 2}$ \\
\hline 00 & 300 & 5.5 & & & & & & 2.9 \\
\hline 01 & 295 & 6.3 & & & & & & 2.8 \\
\hline 02 & 290 & 4.9 & & & & & & 2.9 \\
\hline 03 & 290 & 4.6 & & & & & & 3.0 \\
\hline 04 & 290 & 4.4 & & & & & & 2.9 \\
\hline 05 & 290 & 4.2 & & & & & & 3.0 \\
\hline 06 & 290 & 5.5 & 260 & $-\infty$ & 120 & 2.5 & & 3.1 \\
\hline 07 & 300 & 6.6 & 240 & 4.2 & 120 & 2.8 & & 3.1 \\
\hline 08 & 320 & 7.1 & 230 & 4.4 & 120 & 3.3 & & 2.9 \\
\hline 09 & 340 & 7.6 & 230 & 4.7 & 120 & 3.4 & & 2.9 \\
\hline 10 & 360 & 8.3 & 230 & $(4.9)$ & (110) & (3.6) & & 2.9 \\
\hline 11 & 376 & 8.6 & - & - & $(120)$ & $(3,6)$ & & 2.8 \\
\hline 12 & 375 & 9.1 & -- & - & (115) & $(3.6)$ & & 2.8 \\
\hline 13 & 370 & 9.2 & $\cdots$ & -- & - & $(3.6)$ & & 2.8 \\
\hline 14 & 360 & 9.3 & 230 & $(5.0)$ & $(120)$ & 3.6 & & 2.8 \\
\hline 15 & 350 & 9.3 & 240 & $(4.6)$ & 120 & 3.5 & & 2.9 \\
\hline 16 & 330 & 9.2 & 250 & 4.4 & 120 & 3.3 & & 2.9 \\
\hline 17 & 300 & 8.9 & 250 & 4.2 & 120 & 2.9 & & 2.9 \\
\hline 18 & 280 & 8.6 & 250 & - & 120 & 2.5 & & 3.1 \\
\hline 19 & 240 & 8.0 & & & & & & 3.0 \\
\hline 20 & 255 & 7.1 & & & & & & 3.1 \\
\hline 21 & 270 & 6.0 & & & & & & 2.9 \\
\hline 22 & 290 & 5.6 & & & & & & 2.9 \\
\hline 23 & 300 & 5.5 & & & & & & 2.9 \\
\hline
\end{tabular}

Sweep: $2.12 \dot{M c}_{c}$ to 15.3 Mc in 5 miautes, autonatic operation. 


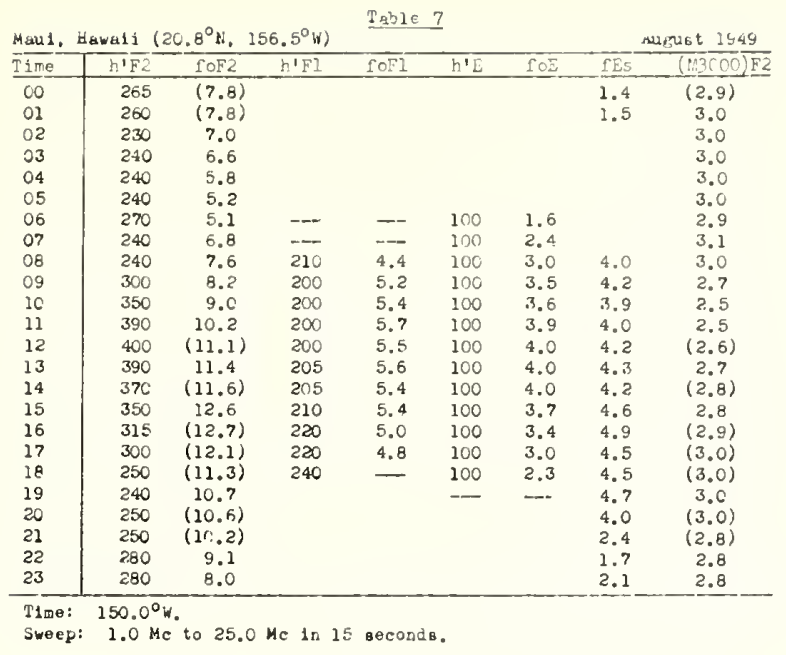

\begin{tabular}{|c|c|c|c|c|c|c|c|c|}
\hline \multicolumn{4}{|c|}{ Gram I. $\left(13.6^{\circ} \mathrm{N}, 144.9^{\circ} \mathrm{E}\right)$} & \multicolumn{3}{|c|}{ Table 9} & \multicolumn{2}{|c|}{ Augast 1949} \\
\hline Time & $h^{1} F^{2}$ & $\mathrm{fOF} 2$ & $h^{\prime} F l$ & roFl. & $n \cdot E$ & fOE & $T E S$ & $(M 3) \bar{C}$ \\
\hline 00 & 275 & $(9.2)$ & & & & & & $(3.0)$ \\
\hline 01 & 245 & 7.1 & & & & & & 3.0 \\
\hline $\mathrm{C} 2$ & 250 & 6.6 & & & & & & 2.8 \\
\hline 03 & 250 & 7.0 & & & & & & 3.0 \\
\hline C4 & 240 & 5.8 & & & & & 1.6 & 3.) \\
\hline 0.5 & 230 & 5.0 & & & & & & 3.2 \\
\hline 06 & 250 & 4.6 & -- & -- & & & 2.1 & 3.1 \\
\hline $0 ?$ & 250 & 7.2 & 220 & 2.7 & 120 & 2.5 & 3.0 & 3.1 \\
\hline 06 & 250 & 8.9 & 220 & -- & 110 & 3.2 & 3.9 & 3.0 \\
\hline 09 & 300 & 9.8 & 210 & -- & 100 & 3.6 & 5.0 & 2.8 \\
\hline 10 & 345 & 10.2 & 210 & 5.0 & 110 & 3.8 & 4.8 & 2.5 \\
\hline 11 & 375 & 10.6 & 200 & 5.0 & $11 C$ & 4.0 & & 2.5 \\
\hline 12 & 395 & $(10.2)$ & 200 & 5.2 & 105 & $(4.0)$ & & 2.3 \\
\hline 13 & 400 & $(10.4)$ & 200 & $(5.0)$ & $-\cdots$ & -- & & $(2,4)$ \\
\hline 14 & 380 & $(10.6)$ & 210 & 5.2 & 110 & 4.0 & & $(2.5)$ \\
\hline 15 & 360 & $(12.0)$ & 215 & 5.2 & 110 & 3.9 & & 2.6 \\
\hline 16 & 360 & $(11.8)$ & 220 & -- & 110 & 3.6 & 4.7 & $(2.7)$ \\
\hline 17 & 360 & (11.3) & 230 & -- & 110 & - & 5.0 & 2.8 \\
\hline 18 & 370 & (11.3) & 255 & - & $\cdots$ & - & 5.0 & $(2.7)$ \\
\hline 19 & 315 & $(11.0)$ & & & & & 2.2 & $(2.8)$ \\
\hline 20 & 350 & $(10.2)$ & & & & & & $(2.6)$ \\
\hline 21 & 300 & $(9.8)$ & & & & & & $\cdots$ \\
\hline 22 & 290 & $(9.7)$ & & & & & 1.6 & $(2.9)$ \\
\hline 23 & 300 & $(8.2)$ & & & & & 2.0 & - \\
\hline
\end{tabular}

Swenp: $1.0 \mathrm{Mc}$ to 25.0 He in 15 reconde.

\begin{tabular}{|c|c|c|c|c|c|c|c|c|}
\hline \multicolumn{4}{|c|}{ Fela ayra I. $\left(5.9^{\circ} \mathrm{H}, 1 \in 2.2^{\circ} \mathrm{H}\right)$} & Table & & & \multirow{2}{*}{\multicolumn{2}{|c|}{$\frac{\text { AMERBt }}{\Gamma E S}-\frac{1949}{(11300)}$}} \\
\hline Time & $h^{\prime} F 2$ & foF 2 & $h^{\prime} F I$ & foF 1 & $h^{\prime} E$ & IOE & & \\
\hline 00 & 250 & 10.7 & & & & & & 2.8 \\
\hline 01 & $\approx 50$ & 10.2 & & & & & & 2.8 \\
\hline 02 & 250 & 9.2 & & & & & & 2.9 \\
\hline 03 & 250 & 8.3 & & & & & 1.7 & 2.9 \\
\hline 04 & 240 & 6.6 & & & & & 2.0 & 3.0 \\
\hline 05 & 250 & 4.6 & & & & & 2.0 & 3.0 \\
\hline 06 & 275 & 4.4 & & & 150 & 1.3 & 2.6 & 2.8 \\
\hline 07 & 250 & 7.0 & & & 120 & 2.5 & 3.2 & 2.8 \\
\hline 08 & 250 & 8.6 & -- & $\cdots$ & 115 & 3.2 & & 2.6 \\
\hline 03 & 240 & 9.2 & 225 & --- & 120 & 3.6 & & 2.4 \\
\hline 10 & 370 & 9.6 & 220 & 5.0 & 120 & 3.8 & & 2.3 \\
\hline 11 & 390 & 10.0 & 220 & 5.1 & 120 & 3.8 & & 2.2 \\
\hline 12 & 390 & 10.4 & 210 & - & 120 & -- & & 2.2 \\
\hline 13 & 410 & 10.8 & 220 & 5.3 & 120 & - & & 2.2 \\
\hline 14 & 400 & 11.4 & 220 & 5.3 & 120 & 4.0 & & 2.2 \\
\hline 15 & 390 & 11.5 & 220 & 5.0 & 120 & 3.7 & & 2.2 \\
\hline 16 & 340 & 11.4 & 230 & - & 120 & 3.5 & & 2.3 \\
\hline 17 & 250 & 11.2 & -- & --- & 120 & 3.0 & 3.7 & 2.3 \\
\hline 18 & 280 & 11.1 & & & 120 & 2.1 & 3.8 & 2.3 \\
\hline 19 & 330 & 10.5 & & & & & 3.0 & 2.2 \\
\hline 20 & 350 & 9.7 & & & & & & 2.2 \\
\hline 21 & 310 & 10.0 & & & & & & 2.4 \\
\hline 22 & 275 & 10.0 & & & & & 2.2 & 2.6 \\
\hline 23 & 250 & 11.5 & & & & & 1.8 & 2.7 \\
\hline
\end{tabular}

Sweep: $1.0 \mathrm{Mc}$ to $13.0 \mathrm{Mc}$ in 1 minute 36 seconds, automatle operat1on: $13.0 \mathrm{Mc}$ to $18.0 \mathrm{Mc}$, mantal operation.

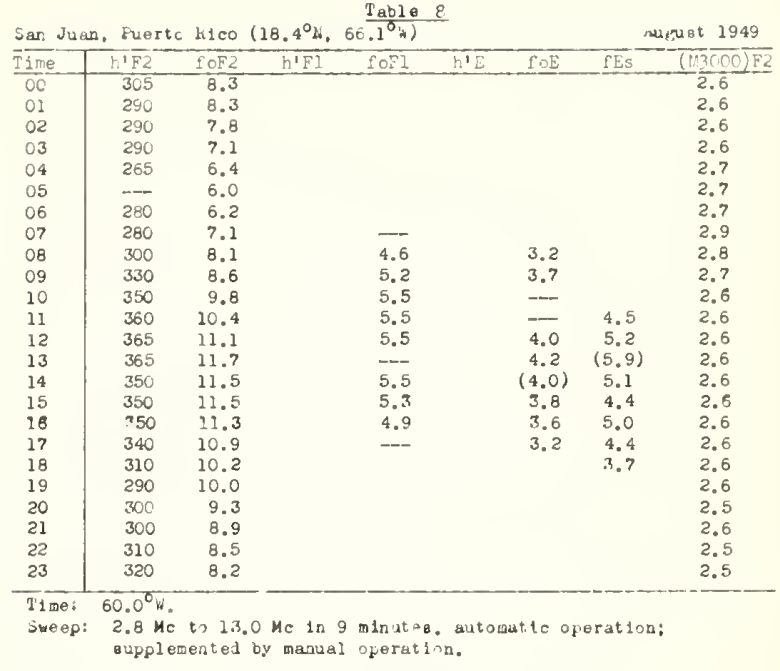

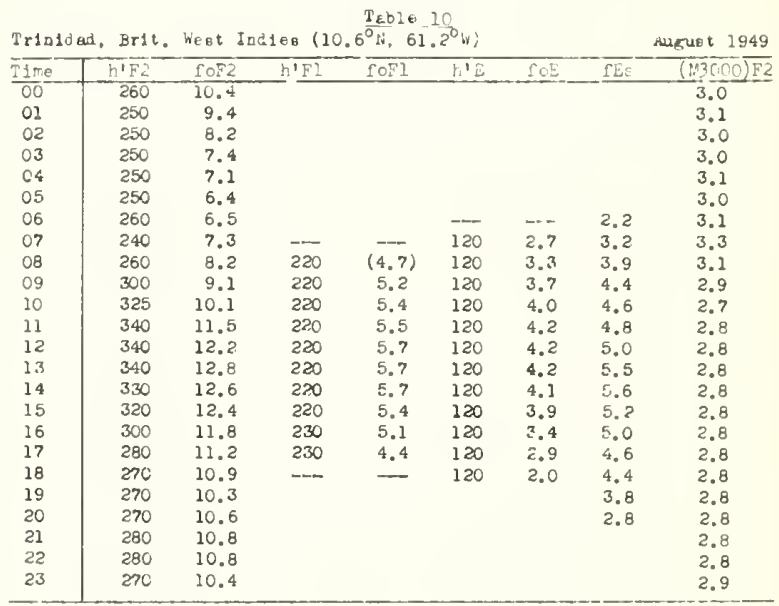

T1mo: $60.0^{\circ} \mathrm{h}$.

Sweep: $1.5 \mathrm{Mc}$ to $18.0 \mathrm{Mc}$, manual operstion.

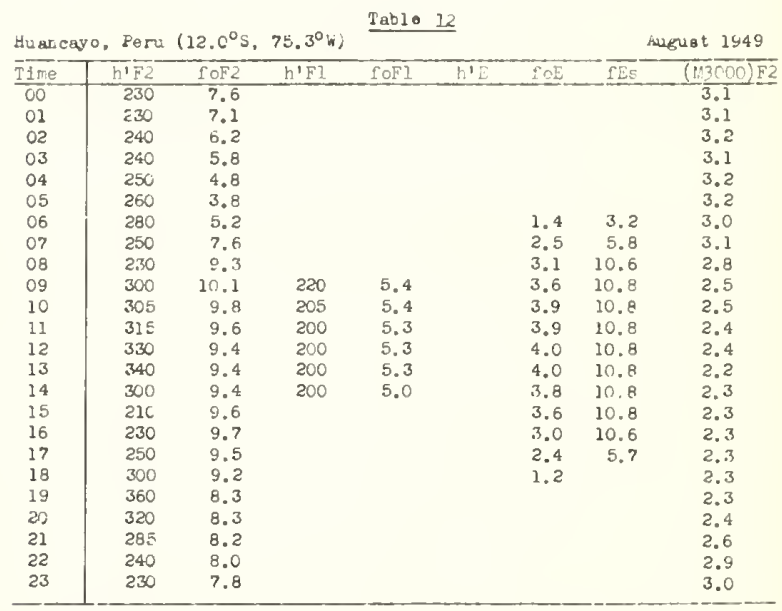

TIme: $75.0^{\circ} \mathrm{s}$.

Sweep: 0.5 Mc to $16.0 \mathrm{Mc}$ in 15 minutre, automatis opeation. 


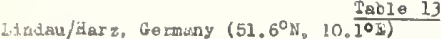

\begin{tabular}{|c|c|c|c|c|c|c|c|}
\hline ine & $h^{\prime} F 2$ & IOF2 & $h^{\prime} F 1$ & fOEI & $h^{\prime} E$ & foE & $1 E s \quad(13000) E^{2}$ \\
\hline 60 & 280 & 6.8 & & & & & 3.4 \\
\hline 01 & 280 & 6.4 & & & & & 3.4 \\
\hline $\mathrm{O} 2$ & 280 & 6.1 & & & & & 3.1 \\
\hline 03 & 290 & 5.8 & & & & & 3.3 \\
\hline 04 & 270 & 5.8 & & & 100 & 1.2 & 3.4 \\
\hline 06 & 270 & 6.4 & 240 & & 110 & 2.9 & 3.6 \\
\hline 06 & 300 & 6.8 & 230 & 4.1 & 100 & 2.4 & $3+9$ \\
\hline 07 & 300 & 7.5 & 230 & 4.6 & 100 & 2.8 & 4.4 \\
\hline 08 & 310 & 7.2 & 220 & 4.7 & 100 & 3.1 & \\
\hline 09 & 320 & 7.4 & 210 & 5.0 & 100 & 3.3 & \\
\hline 10 & 340 & 7.6 & 220 & 5.1 & 100 & 3.4 & 5.6 \\
\hline 11 & 340 & 7.6 & 210 & 5.2 & 100 & 3.5 & 5.6 \\
\hline 12 & 340 & 7.6 & 220 & 5.2 & 100 & 3.6 & \\
\hline 33 & 350 & 7.4 & 220 & 5.4 & 100 & 3.6 & \\
\hline 14 & 350 & 7.3 & 220 & 5.2 & 100 & 3.6 & \\
\hline 15 & 350 & 7.1 & 210 & 5.0 & 100 & 3.4 & \\
\hline 36 & 340 & 7.1 & 230 & 4.9 & 300 & 3.3 & \\
\hline 17 & 300 & 7.2 & 210 & 4.6 & 100 & 3.0 & \\
\hline 18 & 300 & 7.1 & 220 & 4.3 & 100 & 2.8 & 4.3 \\
\hline 19 & 270 & 7.6 & 240 & & 110 & 2.3 & 4.0 \\
\hline 20 & 260 & 7.7 & & & 100 & 1.8 & 3.6 \\
\hline 21 & 260 & 7.6 & & & & & \pm .2 \\
\hline 22 & 260 & 7.5 & & & & & 3.5 \\
\hline 23 & 250 & 7.4 & & & & & 3.2 \\
\hline
\end{tabular}

Tine: $15.0^{\circ} \mathrm{E}$

Swop: $1.0 \mathrm{Mc}$ to $16.0 \mathrm{Mc}$ in 12 minuter.
Table 14

Jobangesburg. Union of 3. Africa $\left(26.2^{\circ} \mathrm{S}, 28,00 \mathrm{~F}\right)$

\begin{tabular}{|c|c|c|c|c|c|c|c|c|}
\hline$\frac{\text { Joban }}{1 \mathrm{n} e}$ & burg. & foF2 & $h^{\prime} F 1$ & SOEI & $h^{\prime} E$ & foE & IEs & $\frac{J 49}{(M 3000) E 2}$ \\
\hline 00 & $(260)$ & 2.8 & & & & & $\lambda .8$ & 2.8 \\
\hline 01 & $(280)$ & 2.9 & & & & & & 2.8 \\
\hline 02 & $(270)$ & 3.0 & & & & & 1.7 & 2.9 \\
\hline 03 & (250) & 2.9 & & & & & 3.4 & 3.0 \\
\hline 04 & $(240)$ & 2.8 & & & & & 3.7 & 3.0 \\
\hline$O B$ & $(250)$ & 2,6 & & & & & 3.4 & 2,9 \\
\hline 06 & $(250)$ & 2.7 & & & 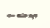 & if & 3.4 & 3.0 \\
\hline 07 & 830 & 5.1 & & & - & 2.8 & & 3.3 \\
\hline 08 & 230 & 7.4 & - & - & 120 & $\langle 2.6\rangle$ & & 3.3 \\
\hline $0 \%$ & 240 & 8.2 & 320 & -- & 110 & $(3.1)$ & & $(3.3)$ \\
\hline 10 & 250 & 9.0 & 220 & + & 110 & 3.4 & & 3.8 \\
\hline 11 & aso & 8.3 & 220 & 4.4 & 110 & $(3.6)$ & & 3.2 \\
\hline 12 & 260 & 9.1 & 210 & 4.8 & 110 & 3.6 & & 3.0 \\
\hline 13 & 260 & 9.3 & 210 & 4.6 & 120 & $(3.6)$ & 3,9 & 3.0 \\
\hline 14 & 270 & 9.3 & 225 & 5.0 & 110 & 3.5 & 3. 8 & 3.0 \\
\hline 25 & 260 & 9.0 & 220 & 4.4 & 110 & $(3,3)$ & 3.8 & 3.0 \\
\hline 16 & 250 & 8.9 & 230 & --- & 110 & 2.9 & 3.5 & 3.0 \\
\hline 17 & 230 & 9.2 & & & 210 & 3.3 & 3.1 & 3.2 \\
\hline 18 & 2) 0 & 7.4 & & & & & 2.5 & 3.3 \\
\hline 19 & 320 & 5.0 & & & & & 3.4 & 3.2 \\
\hline 20 & 230 & 3.9 & & & & & 2.0 & 3,2 \\
\hline 21 & $(240)$ & 3.1 & & & & & 2.0 & 3.1 \\
\hline 22 & 260 & 3.8 & & & & & 1.6 & 3.0 \\
\hline 23 & $(250)$ & 2.9 & & & & & 1.6 & 2.9 \\
\hline
\end{tabular}

Swoup: 1.0 Mo to 25.0 ko in ? exconile.

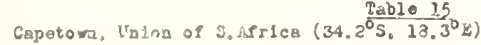

\begin{tabular}{|c|c|c|c|c|c|c|c|c|}
\hline Time & $h^{1} F 2$ & IOF2 & $\mathrm{h}^{\prime} \mathrm{Fl}$ & FOFI & $h^{\prime} E$ & fOE & fEs & $(M 3000) E 2$ \\
\hline 00 & - & 2.6 & & & & & & 2.9 \\
\hline 0.2 & $-\infty$ & 2.7 & & & & & & 2.8 \\
\hline 02 &.- & 2.8 & & & & & & 2.8 \\
\hline 03 & $(260)$ & 3.0 & & & & & & 2.9 \\
\hline 04 & 250 & 2.9 & & & & & & 3.0 \\
\hline 05 & (250) & 2.7 & & & & & & 3.0 \\
\hline 06 & $(240)$ & 2.7 & -- & - & & & & 2.9 \\
\hline 07 & $(250)$ & 2.7 & - & -- & - & E & & 2.9 \\
\hline 08 & 230 & 5.6 & --- & $\cdots$ & --- & (2.8) & & 3.2 \\
\hline OS & 230 & $(7.6)$ & 225 & $-\infty$ & 110 & 2.5 & & 3.3 \\
\hline 10 & 240 & $(8.0)$ & 220 & -- & 110 & $(3.0)$ & & 3.2 \\
\hline 11 & 255 & $(8.5)$ & 210 & --- & 110 & $(3.2)$ & & $(3.2)$ \\
\hline 12 & 260 & $(8,8)$ & 200 & - & 110 & $\cdots$ & & $(3,0)$ \\
\hline 13 & 270 & 9.6 & 210 & --- & 110 & $\cdots$ & & 3.0 \\
\hline 14 & 270 & 9.8 & 220 & $\ldots$ & 120 & -- & & 3.0 \\
\hline 15 & 260 & 9.4 & 230 & $-\cdots$ & 110 & $(3.0)$ & 3.3 & 3.0 \\
\hline 16 & 255 & 94 & 240 & $-\ldots$ & 110 & 2.8 & 3.2 & 3.0 \\
\hline 17 & 240 & 9.5 & - & --- & 110 & 2.5 & 2.6 & 3.2 \\
\hline 18 & 220 & 7.5 & & & $\cdots$ & -- & & $(3.2)$ \\
\hline 19 & 220 & $(5.7)$ & & & & & & $(3.2)$ \\
\hline 20 & 220 & 4.0 & & & & & & $(3.2)$ \\
\hline 21 & 220 & 2.8 & & & & & & 3.2 \\
\hline 22 & $(240)$ & 2.8 & & & & & & 3.0 \\
\hline 23 & $(260)$ & 2.4 & & & & & & 2.9 \\
\hline
\end{tabular}

Tis:00: $30.0^{\circ} \mathrm{z}$

Sweep: 1.0 Ho to $15.0 \mathrm{Hc}$ in 7 eecrinds.

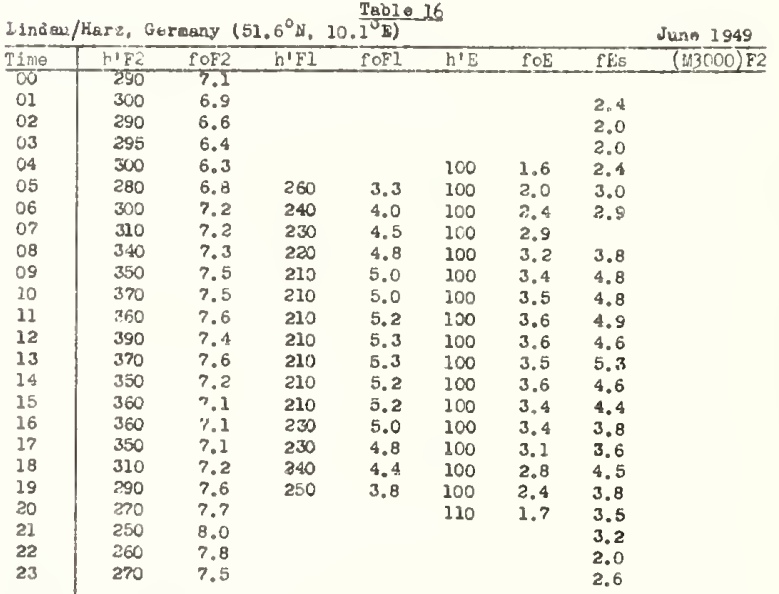

Time: $15.0^{\circ}$.

weep: 1.0 the to 16.0 Me la 12 oinutes.

\begin{tabular}{|c|c|c|c|c|c|c|c|c|}
\hline \multicolumn{3}{|c|}{ Welk kare1, Japan $\left(45.4^{\circ} \mathrm{l}\right.$} & \multicolumn{4}{|c|}{ Table 17} & \multicolumn{2}{|r|}{ June 1949} \\
\hline $1 \lim \theta$ & $h^{\prime} F 2$ & foF2 & $h^{\prime} F I$ & fOFI & $h^{\prime} E$ & foE & $\mathrm{fEs}$ & $(M 3000) E 2$ \\
\hline 00 & 300 & 7.3 & & & & & 2.9 & 2.6 \\
\hline 0.1 & 300 & 7.0 & & & & & 2.4 & 3.6 \\
\hline ca & 300 & 7.1 & & & & & 2.7 & 2.7 \\
\hline 03 & 300 & 6.7 & & & & & 1.9 & 2.6 \\
\hline 04 & 295 & 6.7 & -- & - & -- & 3.8 & 1.9 & 2.6 \\
\hline 05 & 300 & 7.2 & 260 & 3.6 & 100 & 2.3 & 3.1 & 2.7 \\
\hline$O G$ & 320 & 7.8 & 255 & 4.4 & 100 & 2.8 & 4.1 & 2.8 \\
\hline 07 & 335 & 3.1 & 260 & 4.5 & 100 & 3.2 & 5.2 & 2.8 \\
\hline 08 & 325 & 8.2 & 260 & 4.9 & 100 & 3.5 & 5.5 & 2.8 \\
\hline 09 & 3.40 & 7.9 & 220 & 5.0 & 100 & 3.6 & 6.0 & $(2.8)$ \\
\hline 10 & 380 & 7.7 & 215 & 5.0 & 100 & 3.7 & 5.8 & 2.7 \\
\hline 11 & 390 & 8.0 & 220 & 3.2 & 100 & $(3,6)$ & 5.8 & (2.7) \\
\hline 12 & 390 & 8.0 & 250 & 5.2 & 100 & 3.8 & 5.6 & 2.6 \\
\hline 13 & 390 & 7.8 & 260 & 5.2 & 100 & $(3.7)$ & 5.4 & 2.7 \\
\hline 14 & 390 & 7.6 & 240 & 5.0 & 100 & 3.7 & 5.5 & 2.6 \\
\hline 15 & 370 & 7.8 & 245 & 5,0 & 100 & 3.5 & 5.2 & 2.7 \\
\hline 16 & 340 & 7.9 & 250 & 4.7 & 100 & 3.3 & 4.9 & 2.8 \\
\hline 17 & 315 & 7.8 & 250 & $(4.5)$ & 100 & 2.9 & 5.1 & 2.9 \\
\hline 18 & 310 & 7,4 & 260 & -- & 105 & 2.4 & 5.4 & 2.8 \\
\hline 19 & 300 & 7.9 & 250 & $-\cdots$ & 110 & $(1,8)$ & 4.2 & 2.8 \\
\hline 20 & 300 & 7.5 & & & & & 3.8 & 2.8 \\
\hline 21 & 295 & $(7,5)$ & & & & & 3.7 & 2.7 \\
\hline 22 & 300 & 7.1 & & & & & 3.3 & 2.7 \\
\hline $2 z$ & 300 & 7.2 & & & & & 3.4 & 2.6 \\
\hline
\end{tabular}

Swe op: $2.0 \mathrm{kc}$ to $17.0 \mathrm{Mc}$ in 15 minutes, manurl operation.
Troole 18

\begin{tabular}{|c|c|c|c|c|c|c|c|c|}
\hline Pukaura, & \multicolumn{3}{|c|}{$J_{\text {apan }}\left(40.6^{\circ} \mathrm{N}, 139.9^{\circ} \mathrm{E}\right)$} & \multicolumn{4}{|c|}{$20010 \quad 18$} & June 1949 \\
\hline $\operatorname{Tin} \theta$ & h'F2 & foF 2 & $h^{\prime} F 1$ & fOFl & $h^{2} E$ & foE & fES & $(M 3 0 0 0 \longdiv { F 2 }$ \\
\hline 00 & 300 & 7.9 & & & & & 3.6 & 2.7 \\
\hline 01 & 300 & 8.0 & & & & & 3.0 & 2.7 \\
\hline 02 & 300 & 7.6 & & & & & 3.0 & 2.8 \\
\hline 03 & 300 & 7.2 & & & & & 3.0 & 2.7 \\
\hline 04 & 290 & 7,2 & & & $-\infty$ & --- & 3.1 & 2.7 \\
\hline 05 & 285 & 7.4 & 250 & -- & 110 & 2.3 & 3.2 & 2.8 \\
\hline 06 & 295 & 7.9 & 240 & - & 110 & 2.8 & 4.4 & 2.8 \\
\hline 07 & 310 & 8.2 & 245 & 4.9 & 110 & 3.2 & 5.6 & 2.8 \\
\hline 08 & 310 & 8.4 & 220 & $\longrightarrow$ & 110 & 3.4 & 6.2 & 3.8 \\
\hline 09 & 320 & 8.3 & -- & -- & 210 & 3.4 & 6.8 & 2.8 \\
\hline 10 & 375 & 8.0 & --- & 5.6 & 110 & $-\infty$ & 5.8 & 2.7 \\
\hline 11 & 370 & 8.4 & -- & 5.6 & 110 & - & 8.6 & 2.7 \\
\hline 12 & 330 & 8.4 & -- & $(5.6)$ & 210 & $\cdots$ & 7.0 & 2.7 \\
\hline 13 & 375 & 8.4 & $\cdots$ & 5.6 & 110 & 3.8 & 6.2 & 2.8 \\
\hline 14 & 370 & 3.6 & $\because$ & 5.6 & 110 & -- & 5.8 & 2. 8 \\
\hline 15 & 360 & 8.8 & 230 & 5.2 & 110 & --- & 5.2 & 2.8 \\
\hline 16 & 340 & 8.5 & 240 & 5.0 & 110 & 3.4 & 5.4 & 2.8 \\
\hline 17 & 315 & 8.6 & 240 & 4.5 & 110 & 3.0 & 4.9 & 2.8 \\
\hline 18 & $30 n$ & e. 4 & 250 & -- & 120 & 2.6 & 4.7 & 2.9 \\
\hline 19 & 280 & 8.5 & - & --- & -- & -- & 3.8 & 2.9 \\
\hline 20 & 285 & 8.4 & & & & & 5.8 & 2.8 \\
\hline 21 & 300 & 8.1 & & & & & 4.8 & 2.7 \\
\hline 22 & 315 & 8.3 & & & & & 4.6 & 2,7 \\
\hline 23 & 305 & 8.1 & & & & & 4.1 & 2.7 \\
\hline
\end{tabular}

Sweopi $1.0 \mathrm{Kc}$ to $17.0 \mathrm{Kc}$ in 16 minutav, manual operation. 
$\underline{T a b 10} 19$

\begin{tabular}{|c|c|c|c|c|c|c|c|c|}
\hline $5 h 1 b e$ & $J$ apan & $37.9^{\circ} \mathrm{N}$ & $139.3^{\circ}$ & & & & & Turse 1949 \\
\hline$\overline{\mathrm{T}} 1 \mathrm{me}$ & $h^{\prime} F 2$ & f०F2 & $h^{\prime} F I$ & foFl & $h^{\prime} E$ & $f \circ E$ & $\mathrm{fEs}$ & $(\mathrm{M} 3000) \mathrm{F2}$ \\
\hline 00 & 300 & 8.3 & & & & & 3.7 & 2.8 \\
\hline 01 & 280 & 8.4 & & & & & 3.6 & 2.8 \\
\hline 02 & 270 & 8.0 & & & & & 3.5 & 2.9 \\
\hline 03 & 260 & 7.8 & & & & & 3.6 & 2.8 \\
\hline 04 & 285 & 7.3 & & & & & 3.2 & 2.9 \\
\hline 05 & 270 & 7.6 & 225 & $-\infty$ & 115 & 2.0 & 3.7 & 3.0 \\
\hline 06 & 270 & 8.6 & 210 & --- & 100 & 2.7 & 4.8 & 2.9 \\
\hline 07 & 275 & 8.4 & 210 & 4.4 & 100 & 3.2 & 5.6 & 3.0 \\
\hline 08 & 280 & 8.2 & 220 & 5.0 & 100 & 3.4 & 6.9 & 3.0 \\
\hline 09 & 300 & 8.4 & 220 & $\ldots$ & 100 & 3.6 & 8.8 & 2.9 \\
\hline 10 & 330 & 8.4 & 230 & 5.6 & 100 & 3.8 & 7.6 & 2.8 \\
\hline 11 & 330 & 8.8 & 205 & 5.5 & 100 & 4.0 & 9.0 & 2.8 \\
\hline 12 & 340 & 9.0 & 210 & 5.4 & 100 & -- & 8.5 & 2.8 \\
\hline 13 & 330 & 8.8 & 205 & 5.4 & 100 & - & 7.4 & 2.8 \\
\hline 14 & 320 & 9.2 & 200 & 5.4 & 100 & 3.8 & 7.3 & 2.9 \\
\hline 15 & 310 & 9.3 & 205 & 5.2 & 100 & 3.6 & 6.0 & 2.9 \\
\hline 16 & 300 & 9.1 & 220 & 4.8 & 100 & 3.3 & 6.5 & 2.9 \\
\hline 17 & 295 & 9.3 & 215 & 4.4 & 100 & 3.1 & 6.0 & 3.0 \\
\hline 18 & 270 & 9.0 & 230 & -- & 100 & 2.4 & 5.1 & 3.0 \\
\hline 19 & 250 & 8.9 & -- & --- & -- & 1.9 & 3.9 & 3.0 \\
\hline 20 & 255 & 8.5 & & & & & 4.0 & 2.9 \\
\hline 21 & 270 & 8.5 & & & & & 4.2 & 2.7 \\
\hline 22 & 290 & 8.4 & & & & & 4.4 & 2.8 \\
\hline 23 & 290 & 8.4 & & & & & 4.4 & 2.8 \\
\hline
\end{tabular}

T1me: $135.0^{\circ} \mathrm{B}$

Sweop: $1.0 \mathrm{Mc}$ to $17.0 \mathrm{Mc}$ in 15 minutas, manual operation.

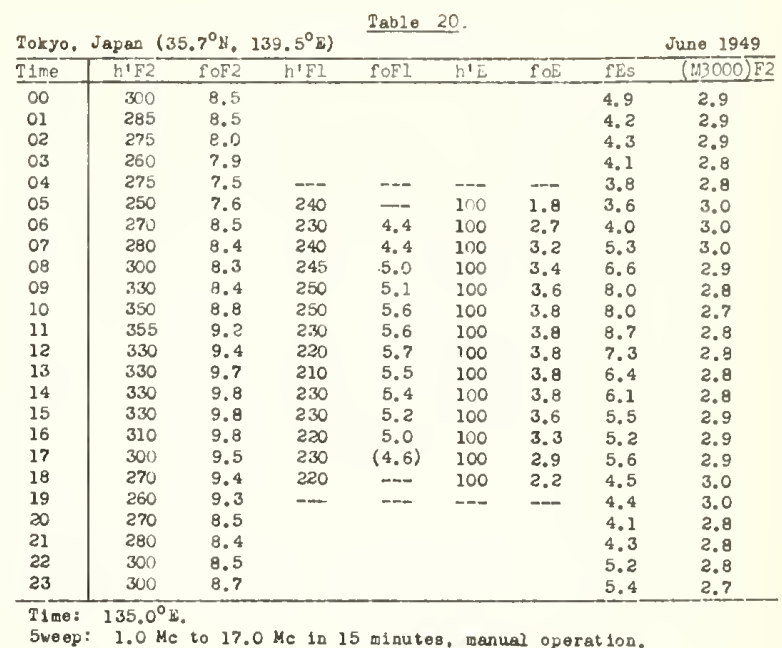

Table 21

\begin{tabular}{|c|c|c|c|c|c|c|c|c|}
\hline Yamak & - Japg & $\left(31,2^{\circ}\right.$ & $130.6^{6}$ & & & & & June 1949 \\
\hline $\mathrm{T} 1 \mathrm{~m} \theta$ & $h^{\prime} \overline{F 2}$ & foF 2 & $h^{\prime} F 1$ & roFI & $h^{\prime} E$ & $f \cong E$ & fES & $(M 3000) E 2$ \\
\hline$\infty 0$ & 310 & 9.2 & & & & & 4.2 & 2.7 \\
\hline 01 & 300 & 9.2 & & & & & 4.6 & 2.7 \\
\hline 02 & 295 & 9.3 & & & & & 4.6 & 2.8 \\
\hline 03 & 280 & 8.4 & & & & & 3.6 & 2.8 \\
\hline 04 & 280 & 7.6 & & & & & 3.8 & 2.8 \\
\hline 05 & 280 & 7.4 & & & & & 3.4 & 2.8 \\
\hline 06 & 260 & 7.4 & 250 & $\cdots$ & 105 & 2.1 & 3.6 & 3.0 \\
\hline 07 & 270 & 8.2 & 230 & - & 110 & 2.8 & 4.4 & 3.0 \\
\hline 08 & 280 & 8.3 & 235 & - & 100 & 3.3 & 5.2 & 2.9 \\
\hline 09 & 320 & 8.6 & 225 & - & 100 & 3.4 & 7.0 & 2.8 \\
\hline 10 & 340 & 9.0 & 210 & 5.3 & 105 & $(3,6)$ & 6.6 & 2.6 \\
\hline 11 & 400 & 9.6 & 210 & 5.5 & 100 & 3.7 & 7.2 & 2.6 \\
\hline 12 & 390 & 9.8 & $(210)$ & 5.6 & 100 & $(3.9)$ & 6.8 & 2.6 \\
\hline 13 & 380 & 10.3 & 215 & 5.6 & 110 & 4.0 & 8.0 & 2.6 \\
\hline 14 & 380 & 10.5 & 220 & 5.5 & 110 & 3.8 & 6.2 & 2.7 \\
\hline 15 & 350 & 10.7 & 230 & 5.4 & 110 & 3.7 & 5.6 & 2.7 \\
\hline 16 & 340 & 11.0 & 235 & 5.2 & 100 & 3.6 & 6.2 & 2.8 \\
\hline 17 & 320 & 10.9 & 250 & 4.9 & 100 & 3.2 & 4.8 & 2.8 \\
\hline 18 & 300 & 10.6 & 240 & $\ldots$ & 100 & 2.7 & 4.6 & 2.8 \\
\hline 19 & 290 & 10.2 & 260 & -- & $-\cdots$ & $(2.0)$ & 4.8 & 2.9 \\
\hline 20 & 270 & 8.8 & & & & & 4.5 & 2.8 \\
\hline 21 & 290 & 8.8 & & & & & 4.2 & 2.6 \\
\hline 22 & 310 & 8.9 & & & & & 4.2 & 2.7 \\
\hline 23 & 310 & 9.0 & & & & & 4.4 & 2.7 \\
\hline
\end{tabular}

5weep: $1.2 \mathrm{Mc}$ to $18.5 \mathrm{Mc}$ in 15 micutes, manual operation.

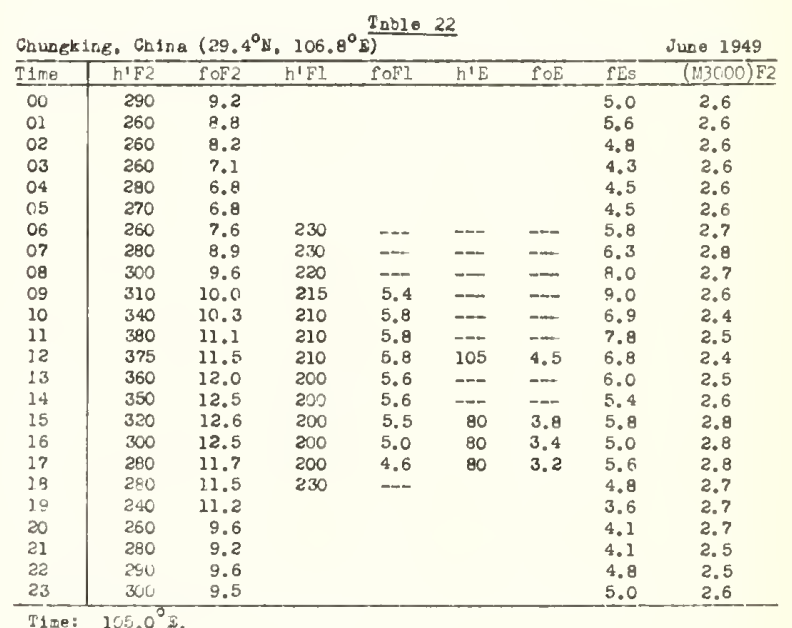

Sweef: $1.5 \mathrm{Mc}$ to $20.0 \mathrm{Mc}$ in 15 minutes, menual operation.

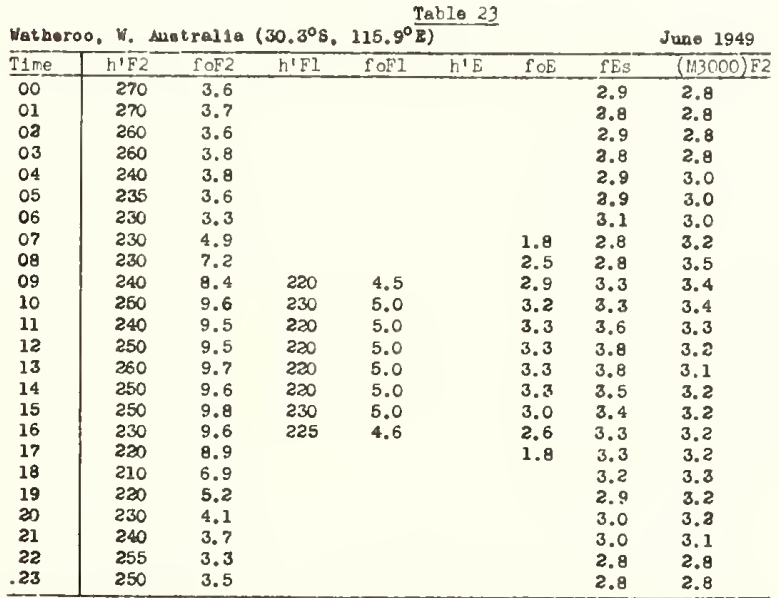

T1ma: $120.0^{\circ} \mathrm{m}$

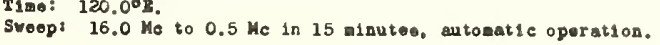

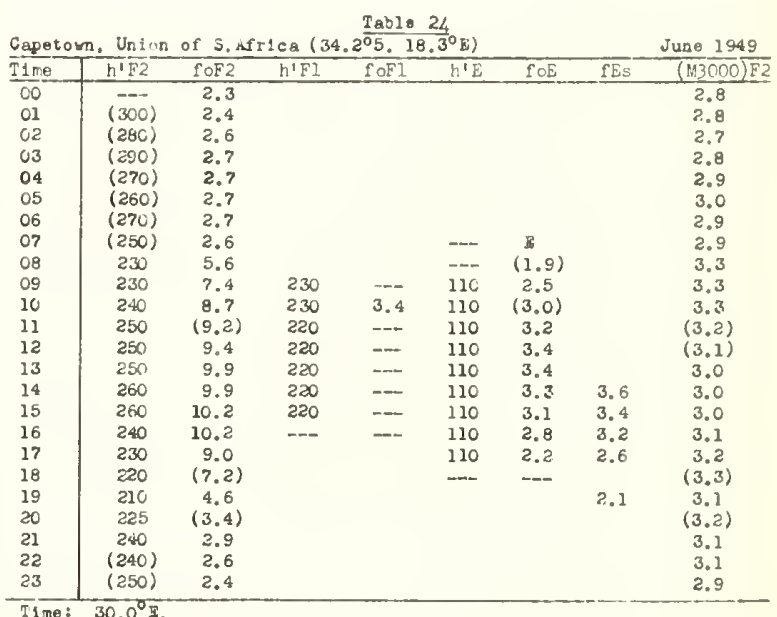

5weep: $1.0 \mathrm{Mc}$ to $15.0 \mathrm{Mc}$ in 7 seconds. 
Table 25

\begin{tabular}{|c|c|c|c|c|c|c|c|c|}
\hline \multicolumn{2}{|c|}{ ChPigkoharah. } & \multicolumn{5}{|c|}{ zouland $\left(43.5^{\circ} \mathrm{s}, \frac{\mathrm{Teblo} 25}{\left.17.7^{\circ} \mathrm{z}\right)}\right.$} & \multicolumn{2}{|r|}{ Jano 1949} \\
\hline Time & $h^{\prime} F^{2}$ & foF 2 & $h^{\prime} F 1$ & IOFI & $h^{\prime} E$ & fOE & fEs & $(113000) E 2$ \\
\hline no & 300 & 3.5 & & & & & 3.8 & 2.6 \\
\hline 01 & 300 & 3.6 & & & & & 3.6 & 2.7 \\
\hline 02 & 300 & 3.7 & & & & & 4.4 & 2.6 \\
\hline 03 & 300 & 3.7 & & & & & 4.4 & 2.6 \\
\hline 04 & 290 & 3.6 & & & & & 4. 2 & 2.7 \\
\hline 05 & 270 & 3.5 & & & & & 4.4 & 2.9 \\
\hline 06 & 260 & 3.2 & & & & & 4.4 & 3.8 \\
\hline $0 ?$ & 250 & 3.4 & & & & & 3.7 & 3.0 \\
\hline 08 & 240 & 6.4 & & & & 3.5 & 4.4 & 3.2 \\
\hline 09 & 240 & 8.0 & - & - & & 2.2 & 4.4 & 3.2 \\
\hline 20 & 350 & 8.8 & - & -- & & 2.7 & 5.0 & 3.2 \\
\hline 11 & 240 & 9.0 & 230 & 4.0 & & 2.9 & 4.6 & 3.2 \\
\hline 13 & 250 & $\theta . \theta$ & 240 & 8.8 & & 3.0 & 5.0 & 3.1 \\
\hline 18 & 250 & 9.4 & 250 & 4. 2 & & 3.0 & 4.9 & $\mathbf{3} .0$ \\
\hline 14 & 250 & 9.4 & 250 & 3.8 & & 2.7 & 6.1 & 3.2 \\
\hline 15 & 250 & 9.0 & -- & $\cdots$ & & 3.4 & B.4 & 3.1 \\
\hline 16 & 24 & 8.1 & - & -- & & 1.7 & 4.4 & 3. $)$. \\
\hline 37 & 240 & 6.8 & & & & $\cdots$ & 3.0 & 3.0 \\
\hline 18 & 250 & 5.6 & & & & & 1.4 & 2.8 \\
\hline 19 & 260 & 5.6 & & & & & 4.0 & 3.0 \\
\hline 20 & 270 & $\$ .0$ & & & & & 3.6 & 3.8 \\
\hline 21 & 280 & 4.4 & & & & & 3.5 & 2.7 \\
\hline 22 & 300 & 4,3 & & & & & 3.0 & 3.7 \\
\hline 23 & 290 & 3.8 & & & & & 3.0 & 2.6 \\
\hline
\end{tabular}

Swo og, 1.0 He to 13.0 He.

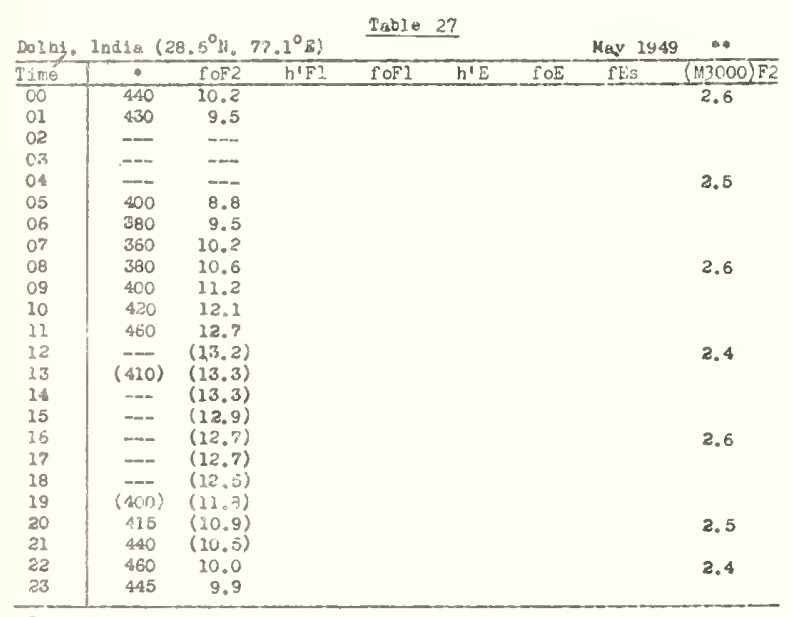

T1me: 2ockl.

Sweep: 1.8 Hc to 16.0 Mc in 5 minutes, namual operation.

lent at 0.83 fope.

- Average vaiues; other columa. medien values.

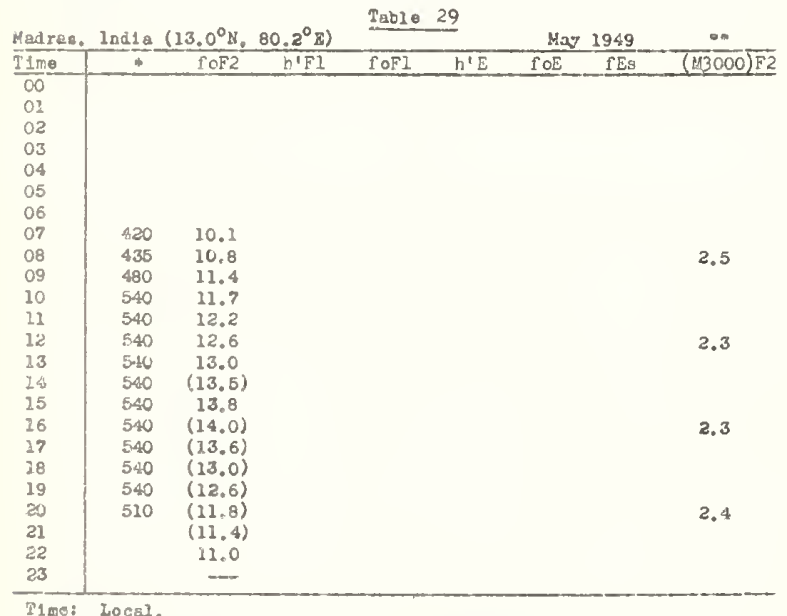

T1 ne: Local.

Syeep: 1.8 Hc to 16.0 Mc in 5 minuter, senusl operation.

- Hol glat at 0.83 fopra.

at Averege values: other colume. pedian values,
Table 26

\begin{tabular}{|c|c|c|c|c|c|c|c|c|}
\hline Lanet & China & $\left(36.1^{\circ} \mathrm{ll}\right.$ & $103.8^{\circ} \mathrm{E}$ & . & & & & May 1949 \\
\hline TIme & $h^{1} F 2$ & foF2 & $\mathrm{h}^{\prime} \mathrm{Fl}$ & SOFI & $h^{\prime} E$ & FoE & $f E E$ & $(113000) E 2$ \\
\hline$\infty$ & 340 & 8.2 & & & & & 3.6 & 2.5 \\
\hline 01 & 340 & 8.4 & & & & & 3.7 & 2.5 \\
\hline 02 & 340 & 8.8 & & & & & 3.8 & 2.5 \\
\hline 03 & 340 & 7.7 & & & & & 3.2 & 2.5 \\
\hline 04 & 340 & 7.2 & & & & & 3.2 & 2.4 \\
\hline $0 b$ & 340 & 7.3 & & & & & 2.6 & 2.4 \\
\hline 06 & 320 & 8.3 & & & & & 3.4 & 2.5 \\
\hline 07 & 320 & 10.5 & 300 & - & 140 & 2.9 & 4.4 & 2.5 \\
\hline 08 & 340 & 11.2 & 320 & - & 140 & 3.4 & 4.6 & 2.6 \\
\hline 09 & 360 & 11.5 & 320 & - & -- & -- & 5.0 & 2.5 \\
\hline 10 & 380 & 11.5 & 320 & - & -- & -- & 5.3 & 2.5 \\
\hline 11 & 380 & 12.5 & 355 & -- & - & -- & 5.2 & 2.3 \\
\hline 12 & 380 & 13.2 & 350 & --- & -- & --- & $(5.2)$ & 2.5 \\
\hline 13 & 380 & 13.5 & 330 & -- & $\cdots$ & $\cdots$ & 4.7 & 2.4 \\
\hline 14 & 380 & 13.2 & 330 & -- & -- & -- & 5.0 & 2.4 \\
\hline 15 & 380 & 13.5 & 340 & -- & $-\infty$ & --- & 4.5 & 2.4 \\
\hline 16 & 360 & 12.3 & 300 & --- & $\cdots$ & --- & 4.5 & 2. 4 \\
\hline 17 & 360 & 13.0 & 320 & -- & 340 & 3.5 & 4.8 & 2.5 \\
\hline 18 & 360 & 11.5 & 300 & -- & $-\cdots$ & -- & 4.8 & 2.5 \\
\hline 19 & 310 & 11.8 & & & & & 4.6 & 2.5 \\
\hline 20 & 290 & $(10.0)$ & & & & & $(4,5)$ & $\langle 3.5\rangle$ \\
\hline 21 & 290 & 8.9 & & & & & $(4.1)$ & 3.5 \\
\hline 22 & 320 & 9.4 & & & & & 3.9 & 2.4 \\
\hline 23 & 330 & 9.0 & & & & & 4.1 & 2.4 \\
\hline
\end{tabular}

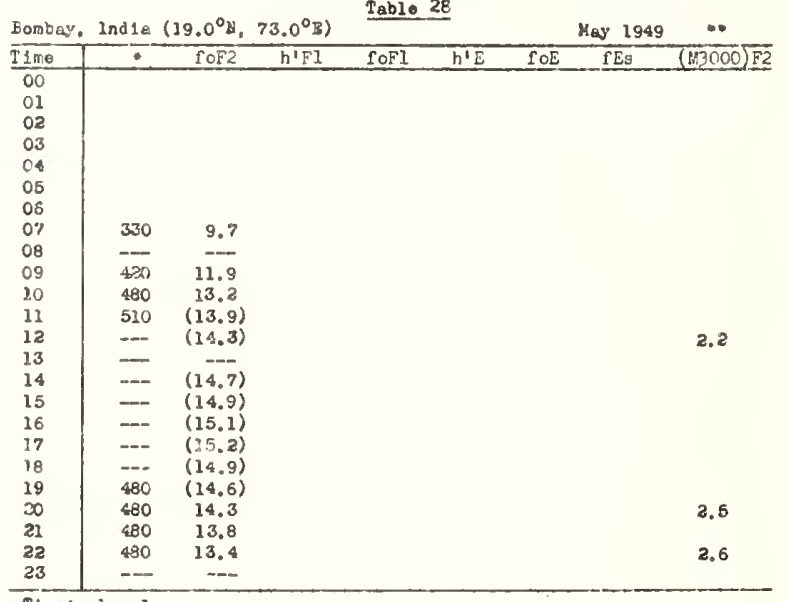

T1 me: Lacel.

Sreep: $1.8 \mathrm{Mc}$ to 16.0 Me in 5 minutes, manual operstion.

- Hel ght at $0.83 \mathrm{fola}$

- Aperape values: othor columa. mediar vajuer.

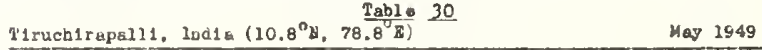

\begin{tabular}{|c|c|c|c|c|c|c|c|c|}
\hline IIme & $\infty$ & IOF2 & $h^{\prime} F I$ & foFl & $h^{\prime} E$ & $I O E$ & rES & $(43000) E 2$ \\
\hline$\infty$ & & & & & & & & \\
\hline 01 & & & & & & & & \\
\hline 02 & & & & & & & & \\
\hline 03 & & & & & & & & \\
\hline 04 & & & & & & & & \\
\hline 05 & & & & & & & & \\
\hline 06 & 360 & 9.0 & & & & & & \\
\hline 67 & .390 & 9.9 & & & & & & \\
\hline 08 & 120 & 31.5 & & & & & & \\
\hline 09 & 510 & 11.6 & & & & & & \\
\hline 10 & 540 & 11.3 & & & & & & \\
\hline 11 & 540 & 11.2 & & & & & & \\
\hline 12 & 540 & 11.5 & & & & & & \\
\hline 13 & 580 & 11.0 & & & & & & \\
\hline 14 & 600 & 11.0 & & & & & & \\
\hline 15 & 620 & 12.2 & & & & & & \\
\hline 16 & 550 & 13.2 & & & & & & \\
\hline 17 & 585 & 12.6 & & & & & & \\
\hline 10 & 540 & 13.8 & & & & & & \\
\hline 19 & 580 & 12.2 & & & & & & \\
\hline 20 & 540 & 11.8 & & & & & & \\
\hline 21 & 510 & 11.5 & & & & & & \\
\hline 22 & 500 & 31.8 & & & & & & \\
\hline 23 & & & & & & & & \\
\hline
\end{tabular}

Thated Local.

Swerp: 1.8 Kc to $16.0 \mathrm{Hc}$ is 5 minuter, menual operation.

"Holght at 0.83 1082. 
Tabla 31

\begin{tabular}{|c|c|c|c|c|c|c|c|c|}
\hline \multicolumn{2}{|c|}{ Caloutta. } & \multicolumn{2}{|c|}{$\left(22.60 \mathrm{~N}, 88.4^{\circ} \mathrm{E}\right)$} & & \multirow{2}{*}{$\frac{\bullet}{f \circ E}$} & \multicolumn{2}{|c|}{ Apr11 1949} \\
\hline Time & $h^{1} \overline{F 2}$ & $\mathrm{fOF} 2$ & h'FI & POFI & $h^{\prime} E$ & & $\mathrm{fEs}$ & $(\mathrm{M} 3000) \overline{F 2}$ \\
\hline 00 & $(240)$ & $(12.4)$ & & & & 1.2 & & $(2.8)$ \\
\hline 01 & & $(11,2)$ & & & & 1.2 & & \\
\hline 02 & & $(10.1)$ & & & & 1.3 & & \\
\hline 03 & -- & $(9.0)$ & & & & 1.1 & & - \\
\hline 04 & & -- & & & & - & & \\
\hline 05 & & -- & & & & -- & & \\
\hline 06 & $(240)$ & $(8.6)$ & & & & 2.0 & & $(2,9)$ \\
\hline 07 & & 10.6 & & & & 3.1 & & \\
\hline 08 & & 11.0 & & & & 3.6 & & \\
\hline 09 & 270 & 12.4 & & & & 3.8 & & 2.7 \\
\hline 10 & & 12.6 & & & & 4.0 & & \\
\hline 11 & & 12.6 & & & & 4.0 & & \\
\hline 12 & $(240)$ & 12.5 & & & & 3.9 & & 2.8 \\
\hline 13 & & 12.5 & & & & $\ldots$ & & \\
\hline 14 & & 12.5 & & & & - & & \\
\hline 15 & -- & 12.6 & & & & $\cdots$ & & - \\
\hline 16 & & 12.6 & & & & 3.8 & & \\
\hline 17 & & 12.5 & & & & 3.4 & & \\
\hline 18 & $(240)$ & 12.5 & & & & 3.2 & & $(2,8)$ \\
\hline 19 & & $(11.8)$ & & & & $-\cdots$ & & \\
\hline 20 & & $(12.4)$ & & & & 2.0 & & \\
\hline 21 & 240 & 12.0 & & & & 1.5 & & 2.8 \\
\hline 22 & & 12.2 & & & & 1.4 & & \\
\hline 23 & & 12.3 & & & & 1.2 & & \\
\hline
\end{tabular}

T1me: Local.

- Prodably 1acludeo $\mathrm{fE}$ obearvatiung.

\begin{tabular}{|c|c|c|c|c|c|c|c|c|}
\hline \multicolumn{3}{|c|}{ Caloutta, Ind1 a $\left(22.6^{\circ} \mathrm{d}\right.$} & $\left.88.4^{\circ} \mathrm{I}\right)$ & \multicolumn{2}{|c|}{ Table 33} & \multicolumn{3}{|r|}{ August 1948} \\
\hline Time & $h 1 F 2$ & foF2 & $h^{\prime} F l$ & foll & $h^{\prime} \mathrm{I}$ & fOE & $\mathrm{fEs}$ & $(\mathrm{M} 3 \mathrm{COO}) \mathrm{F} 2$ \\
\hline 00 & 330 & 10.2 & & & & 2.7 & & 2.7 \\
\hline 01 & & 9.6 & & & & 3.4 & & \\
\hline 02 & & 8.7 & & & & 2.3 & & \\
\hline 03 & 300 & $(8.2)$ & & & & 2.5 & & $(2.7)$ \\
\hline 04 & & 7.4 & & & & 2.2 & & \\
\hline 05 & & 7.6 & & & & 2.2 & & \\
\hline 06 & 360 & 8.2 & & & & 2.7 & & 2.7 \\
\hline 07 & & 8.9 & & & & 3.0 & & \\
\hline 08 & & 9.8 & & & & 3.6 & & \\
\hline 09 & 360 & 10.8 & & & & 4.2 & & 2.7 \\
\hline 10 & & 11.0 & & & & 4.2 & & \\
\hline 11 & & 21.2 & & & & 4.6 & & \\
\hline 12 & 390 & 11.1 & & & & 4.5 & & 2.4 \\
\hline 13 & & 11.2 & & & & - & & \\
\hline 14 & & 11.8 & & & & -- & & \\
\hline 15 & 390 & 11.2 & & & & - & & $(2,7)$ \\
\hline 18 & & 11.1 & & & & 4.5 & & \\
\hline 17 & & 11.0 & & & & 4.4 & & \\
\hline 18 & 360 & 11.3 & & & & 4.2 & & 2.7 \\
\hline 19 & & 11.1 & & & & 3.8 & & \\
\hline 20 & & 11.0 & & & & 3.0 & & \\
\hline $2 i$ & 360 & 11.0 & & & & 3.0 & & 2.7 \\
\hline 22 & & 10.9 & & & & 3.1 & & \\
\hline 23 & & 10.6 & & & & 2.9 & & \\
\hline
\end{tabular}

- Probably includer file odeervations.

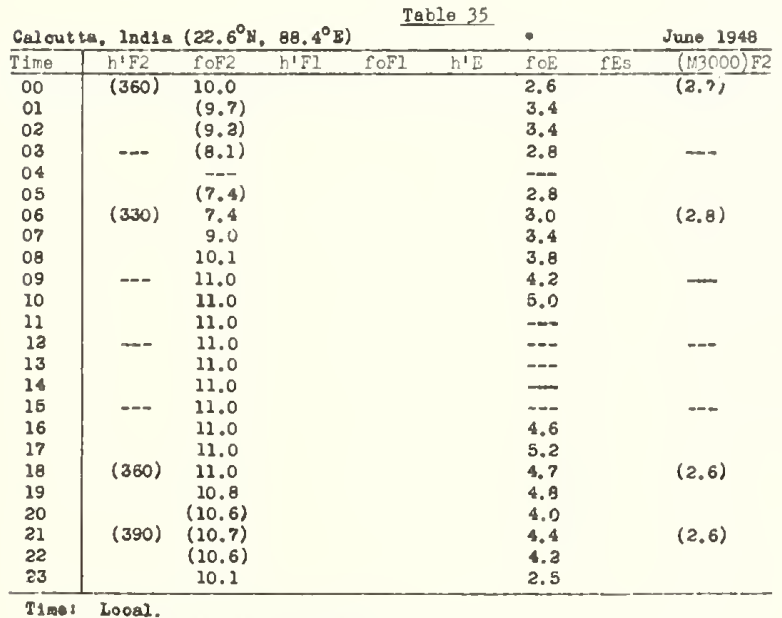

"Prohably 1noluder Ple obeorvation.
Tabla 32

\begin{tabular}{|c|c|c|c|c|c|c|c|c|}
\hline \multirow{2}{*}{ Calcut } & 2. Ind10 & \multicolumn{2}{|c|}{$\left(22.6^{\circ} \mathrm{H}, 88.4^{\circ} \mathrm{E}\right)$} & \multicolumn{2}{|c|}{ Taola 32} & \multirow{2}{*}{$\stackrel{-}{f \circ E}$} & \multicolumn{2}{|c|}{ March 1949} \\
\hline & & $\mathrm{fOF} 2$ & $h^{\prime} F 1$ & foFl & $h^{\prime} c$ & & fES & $(M 3000) \overline{F 2}$ \\
\hline 00 & 240 & 13.0 & & & & 1.3 & & 2.8 \\
\hline 01 & & 12.4 & & & & 1.1 & & \\
\hline 02 & & 11,3 & & & & 1.1 & & \\
\hline 03 & $(210)$ & 8.4 & & & & 1.1 & & $(3.1)$ \\
\hline 04 & & $\ldots$ & & & & -- & & \\
\hline 05 & & $(7.8)$ & & & & 1.1 & & \\
\hline 06 & -- & $(9.1)$ & & & & 2.1 & & -- \\
\hline 07 & & 11.0 & & & & 2.7 & & \\
\hline 08 & & 12.4 & & & & 3.2 & & \\
\hline 09 & 270 & 12.8 & & & & 4.3 & & 2.8 \\
\hline 10 & & 13.2 & & & & 4.0 & & \\
\hline 11 & & 13.2 & & & & 4.1 & & \\
\hline 12 &.- & 13.1 & & & & $\ldots$ & & $(2.6)$ \\
\hline 13 & & $(13.1)$ & & & & - & & \\
\hline 14 & & 13.3 & & & & $\ldots$ & & \\
\hline 15 & --- & 13.1 & & & & $-\infty$ & & --- \\
\hline 16 & & 13.0 & & & & 3.8 & & \\
\hline 17 & & 13.0 & & & & 3.4 & & \\
\hline 18 & 270 & 13.0 & & & & 3.0 & & 2.8 \\
\hline 19 & & 13.6 & & & & 2.9 & & \\
\hline 20 & & 12.4 & & & & 2.5 & & \\
\hline 21 & (210) & $(12.8)$ & & & & 1.5 & & $(2,8)$ \\
\hline 22 & & 13.0 & & & & 1.3 & & \\
\hline 23 & & 14.0 & & & & 1.4 & & \\
\hline
\end{tabular}

Table 34

\begin{tabular}{|c|c|c|c|c|c|c|c|c|}
\hline Calcut & \multicolumn{5}{|c|}{, Ind1a $\left(22,6^{\circ} \mathrm{H}, 88.4^{\circ} \mathrm{Z}\right)$} & \multicolumn{3}{|c|}{ July 1948} \\
\hline Time & $h^{\prime} \overline{E 2}$ & foF2 & $h^{1} F 1$ & roFl & $h^{\prime} E$ & foE & fEs & $(13000,12$ \\
\hline 00 & 345 & 9.9 & & & & 3.0 & & 2.8 \\
\hline 01 & & 9.8 & & & & 1.9 & & \\
\hline 02 & & $(8.9)$ & & & & 2.0 & & \\
\hline 03 & -- & $(7,6)$ & & & & 2.0 & & $m$ \\
\hline 04 & & $\ldots$ & & & & $\rightarrow$ & & \\
\hline 05 & & $(6.8)$ & & & & 2.0 & & \\
\hline 06 & 330 & 7.2 & & & & 2.6 & & 2.8 \\
\hline 07 & & 8.0 & & & & 3.2 & & \\
\hline 08 & & 9.2 & & & & 3.8 & & \\
\hline 09 & 360 & 20.4 & & & & 4.0 & & 2.7 \\
\hline 10 & & 11.0 & & & & 4.0 & & \\
\hline 11 & & 11.2 & & & & 4.2 & & \\
\hline 12 & $(390)$ & 11.0 & & & & - & & 2.6 \\
\hline 13 & & 11.0 & & & & - & & \\
\hline 14 & & 11.1 & & & & -.. & & \\
\hline 15 & 360 & 11.2 & & & & 4.0 & & 3.6 \\
\hline 16 & & 11.0 & & & & 4.0 & & \\
\hline 17 & & 11.0 & & & & 3.6 & & \\
\hline 18 & 360 & 11.0 & & & & 3.3 & & 2.7 \\
\hline 19 & & 10.8 & & & & 3.2 & & \\
\hline 20 & & 10.9 & & & & 3.2 & & \\
\hline 21 & $(360)$ & 10.9 & & & & 3.0 & & 2.9 \\
\hline 22 & & 10.5 & & & & 2.9 & & \\
\hline 23 & & 20.0 & & & & 3.0 & & \\
\hline
\end{tabular}

Time: Local.

- Prodably includea rise obcervationt.

\begin{tabular}{|c|c|c|c|c|c|c|c|c|}
\hline \multirow{2}{*}{ Oalout } & \multicolumn{2}{|c|}{ a, Ind1a $\left(22.8^{\circ} \mathrm{H}\right.$, } & \multicolumn{3}{|c|}{ Tabla 36} & 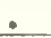 & \multicolumn{2}{|r|}{ May 1948} \\
\hline & $h^{\prime} F 2$ & fOF2 & $h^{\prime F I}$ & fOFI & $h^{\prime} E$ & fOE & $\overline{f E S}$ & $(113000) \overline{F 2}$ \\
\hline$\infty$ & 390 & 10.6 & & & & 3.7 & & 2.6 \\
\hline 01 & & 10.2 & & & & 3.8 & & \\
\hline 08 & & 9.3 & & & & 3.6 & & \\
\hline 03 & $(400)$ & 8.2 & & & & 3.2 & & $(2,4)$ \\
\hline 04 & & 7.6 & & & & 2.6 & & \\
\hline 05 & & 7.8 & & & & 2.8 & & \\
\hline 06 & (375) & 9.0 & & & & 3.0 & & $(2.5)$ \\
\hline 07 & & 0.7 & & & & 3.6 & & \\
\hline 08 & & 10.6 & & & & 3.9 & & \\
\hline 09 & -- & $10 . \mathrm{B}$ & & & & 4.4 & & - \\
\hline 10 & & 11.0 & & & & 4.2 & & \\
\hline 11 & & 11.0 & & & & 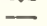 & & \\
\hline 12 & -- & 11.0 & & & & -- & & - \\
\hline 13 & & 11.0 & & & & $-\infty$ & & \\
\hline 14 & & (11.0) & & & & -.- & & \\
\hline 15 & --- & $(11.0)$ & & & & $-\cdots$ & & - \\
\hline 16 & & 11.0 & & & & --- & & \\
\hline 17 & & 11.0 & & & & 6.7 & & \\
\hline 18 & (390) & 11.0 & & & & 6.1 & & $(2,7)$ \\
\hline 19 & & 11.0 & & & & 4.7 & & \\
\hline 20 & & 11.0 & & & & 4.6 & & \\
\hline 21 & 375 & 10.9 & & & & 4.5 & & $(2.6)$ \\
\hline 22 & & 10.8 & & & & 4.4 & & \\
\hline 23 & & 10.8 & & & & 3.9 & & \\
\hline
\end{tabular}

- Probably inoluder rse obsarvation. 


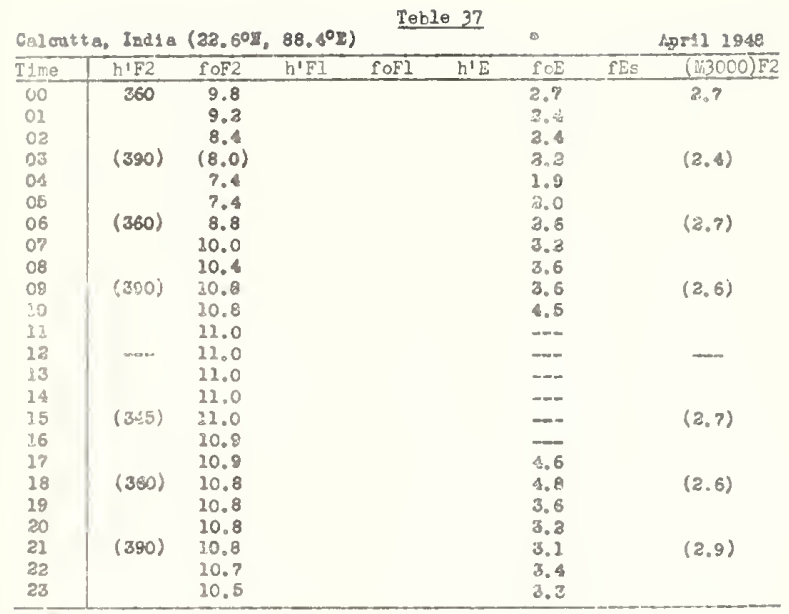

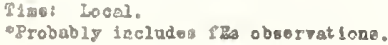

\begin{tabular}{|c|c|c|c|c|c|c|c|c|}
\hline \multicolumn{3}{|c|}{ Colestca, ladis $\left(22.6^{\circ} \mathrm{s}\right.$, } & $\left.88.4^{\circ} \mathrm{E}\right)$ & \multicolumn{2}{|c|}{ Teble 39} & \multirow{2}{*}{$\therefore$} & \multicolumn{2}{|c|}{ Tobruarg 1940} \\
\hline IIme & $\mathrm{h}^{\prime} \mathrm{F2}$ & foF2 & $h^{\prime} F l$ & foFl & $h^{\prime} E$ & & $f E_{9}$ & $( M 3 0 0 0 ) \longdiv { E 2 }$ \\
\hline 00 & 330 & 10.0 & & & & 2.2 & & 3.6 \\
\hline 02 & & 8.5 & & & & 2.2 & & \\
\hline 02 & & 7.8 & & & & 2.0 & & \\
\hline 03 & 300 & 6.3 & & & & 3.4 & & 3,6 \\
\hline 04 & & 5.8 & & & & 2.2 & & \\
\hline 05 & & $(6,0)$ & & & & 1.9 & & \\
\hline 08 & 330 & 7.2 & & & & 2.2 & & 2.7 \\
\hline 07 & & 7.9 & & & & 2.8 & & \\
\hline 08 & & 9.7 & & & & 3.0 & & \\
\hline 09 & 360 & 10.9 & & & & 3.9 & & 2.6 \\
\hline 20 & & 21.0 & & & & 4.0 & & \\
\hline 11 & & $(13,0)$ & & & & 4.2 & & \\
\hline 12 & 360 & 11.0 & & & & 4.2 & & a. 6 \\
\hline 23 & & 21.0 & & & & 4.2 & & \\
\hline 14 & & 11.0 & & & & 3.8 & & \\
\hline 25 & 330 & 11.0 & & & & 3.6 & & 3.5 \\
\hline 26 & & 21.0 & & & & 3.4 & & \\
\hline 17 & & $(12,0\rangle$ & & & & 3.4 & & \\
\hline 28 & 300 & $(10.9)$ & & & & 3.5 & & $(2,5)$ \\
\hline 29 & & $(11.0)$ & & & & 3.3 & & \\
\hline 20 & & $(11,0)$ & & & & 3.0 & & \\
\hline 21 & 300 & $(10.9)$ & & & & 2.0 & & $(3.5)$ \\
\hline 22 & & 20.6 & & & & 3.6 & & \\
\hline 23 & & $(20.6)$ & & & & 2.6 & & \\
\hline
\end{tabular}

- Probably ineludoe sEa cberrtationg.

\begin{tabular}{|c|c|c|c|c|c|c|c|c|}
\hline Cal cet & \multicolumn{2}{|c|}{ Indie $\left(22,6^{\circ} \mathrm{N}\right.$} & \multicolumn{2}{|c|}{$\left.88.4^{\circ} \mathrm{x}\right)$} & & \multirow{2}{*}{$\frac{\bullet}{f \circ E}$} & \multicolumn{2}{|c|}{ Docembar 1947} \\
\hline Time & $h^{+} \mathrm{F2}$ & foE2 & $h^{\prime} E I$ & EOFI & $h^{\prime} E$ & & $f E_{S}$ & $(M 3000) F_{2}$ \\
\hline$\infty$ & 270 & 9.5 & & & & 1.8 & & $2 . ?$ \\
\hline 01 & & 8.4 & & & & 2.7 & & \\
\hline 02 & & 8.2 & & & & 1.6 & & \\
\hline 03 & 270 & 7.4 & & & & 1.6 & & 2.8 \\
\hline 04 & & 6.4 & & & & 2.6 & & \\
\hline os & & 6.6 & & & & 1.8 & & \\
\hline 06 & 300 & 7.5 & & & & 3.0 & & 3.0 \\
\hline 07 & & 9.0 & & & & 2.6 & & \\
\hline$O \theta$ & & 11.8 & & & & 3.0 & & \\
\hline 09 & 330 & 11.2 & & & & 3.4 & & 2.8 \\
\hline 10 & & 12.6 & & & & 4.0 & & \\
\hline 11 & & $(11.6)$ & & & & 4. 2 & & \\
\hline 12 & 352 & 11.6 & & & & 4.2 & & 2.8 \\
\hline 13 & & 12.6 & & & & 4.2 & & \\
\hline 14 & & 11.6 & & & & 4.2 & & \\
\hline 25 & 240 & 11.6 & & & & 4.2 & & 2.6 \\
\hline 16 & & 11.7 & & & & 3.2 & & \\
\hline 27 & & 11.6 & & & & 2.9 & & \\
\hline 18 & 300 & 11.7 & & & & $3 . ?$ & & $\cdots$ \\
\hline 19 & & 11.8 & & & & 3.6 & & \\
\hline 20 & & 11.2 & & & & 2.4 & & \\
\hline 21 & 240 & 20.5 & & & & 3.2 & & 3.0 \\
\hline 22 & & 10.8 & & & & 3.1 & & \\
\hline 23 & & 10.0 & & & & 1.6 & & \\
\hline
\end{tabular}

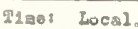

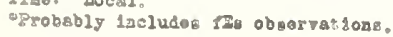

\begin{tabular}{|c|c|c|c|c|c|c|c|c|}
\hline \multicolumn{6}{|c|}{ Onjeurca, inala (22,eon, 88, $\left.0^{\circ} \mathrm{N}\right)$} & \multicolumn{3}{|c|}{ Viareb 2948} \\
\hline Ine & $h^{1} \bar{F} 2$ & fOF2 & $\left.h^{1} F\right]$ & foFl & $h^{1} E$ & $\mathrm{fOE}$ & fEs & $(1 \mathrm { M3 } 0 0 0 \longdiv { \mathrm { F } 2 }$ \\
\hline 00 & 300 & 9.7 & & & & 1.9 & & 2.6 \\
\hline 01 & & 8.7 & & & & 2.0 & & \\
\hline 02 & & 8.2 & & & & 2.0 & & \\
\hline 03 & 330 & 7.0 & & & & 1.9 & & 2.7 \\
\hline 04 & & 5.0 & & & & 3.0 & & \\
\hline 05 & & 6.3 & & & & 3.0 & & \\
\hline 06 & 300 & 7.2 & & & & 2.3 & & 2.6 \\
\hline 07 & & 8.8 & & & & 3.0 & & \\
\hline 08 & & 10.0 & & & & 3.2 & & \\
\hline 09 & 300 & 10.8 & & & & $3 . ?$ & & 2.6 \\
\hline 10 & & 11.0 & & & & 4.8 & & \\
\hline is & & 11.4 & & & & 4.6 & & \\
\hline 23 & 360 & 11.6 & & & & 5.0 & & 3.4 \\
\hline 13 & & 13.0 & & & & 4.3 & & \\
\hline 14 & & $(11.7)$ & & & & 4.0 & & \\
\hline 15 & 360 & 11.5 & & & & 3.6 & & 2.5 \\
\hline 16 & & 11.3 & & & & 3.6 & & \\
\hline 27 & & 11.2 & & & & 3.4 & & \\
\hline 28 & 360 & 11.0 & & & & 3.0 & & 2.6 \\
\hline 38 & & 10.8 & & & & 2.6 & & \\
\hline 20 & & 10.7 & & & & 3.1 & & \\
\hline ¿1 & 300 & 10.6 & & & & 2.0 & & 2.6 \\
\hline 22 & & 10.5 & & & & 3.0 & & \\
\hline 33 & & 10.1 & & & & 1.9 & & \\
\hline
\end{tabular}

Tian wocal.

ofrobably 1zelude. II obeorvatione.

Table 40

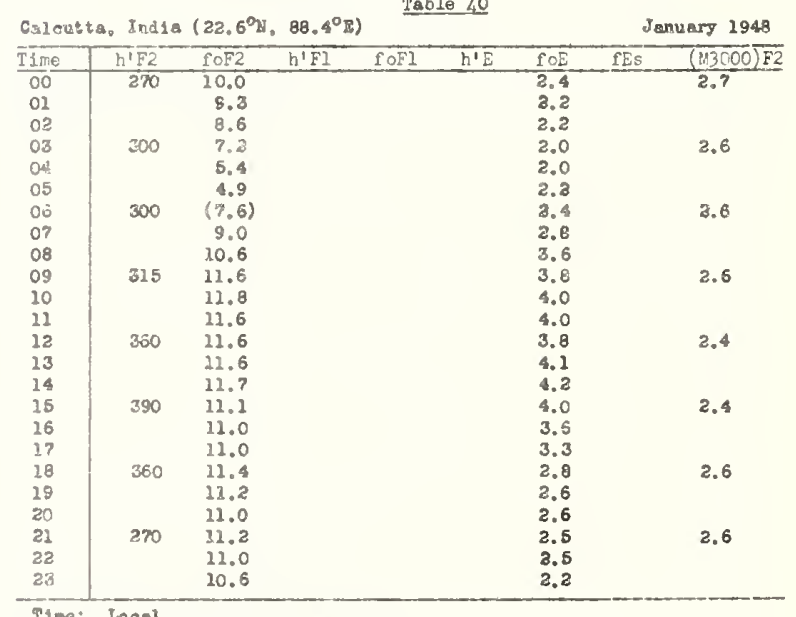

Drobably includes SFa observatione.

Table 42

\begin{tabular}{|c|c|c|c|c|c|c|c|c|}
\hline Caveutts & \multicolumn{5}{|c|}{ Indda $\left(22.6^{\circ} \mathrm{B}, 88.4^{\circ} \mathrm{E}\right)$} & 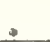 & \multicolumn{2}{|c|}{ Ho7 30006 1949} \\
\hline IIme & $h^{1} E 2$ & fOF2 & $h^{\prime} F I$ & foFl & $h^{1} E$ & foE & $\mathrm{fES}$ & $(113000) 5$ \\
\hline 00 & $(330)$ & 21.0 & & & & 2.2 & & $(3.6)$ \\
\hline 01 & & 21.5 & & & & 3.2 & & \\
\hline 02 & & 11.2 & & & & 2.0 & & \\
\hline 03 & 295 & 9.4 & & & & 2.0 & & 3.7 \\
\hline 04 & & 7.9 & & & & 2.2 & & \\
\hline 05 & & 8.4 & & & & 2.5 & & \\
\hline 06 & 285 & 9.2 & & & & 2.6 & & 2.8 \\
\hline 07 & & 20.8 & & & & 3.0 & & \\
\hline 08 & & 11.8 & & & & 8.3 & & \\
\hline 09 & - & 11.7 & & & & 3.6 & & $(2.6)$ \\
\hline 10 & & 12.8 & & & & 4.1 & & \\
\hline 11 & & 13.9 & & & & 4.0 & & \\
\hline 12 & $(320)$ & 11.9 & & & & 4.7 & & (3.8) \\
\hline 13 & & 12.0 & & & & 4.5 & & \\
\hline 14 & & 12.0 & & & & 4.0 & & \\
\hline 15 & 330 & 11.8 & & & & 3.9 & & 2.6 \\
\hline 16 & & 11.8 & & & & 3.6 & & \\
\hline 18 & & 12.0 & & & & 3.3 & & \\
\hline 18 & 850 & 11.8 & & & & 3.2 & & 2.7 \\
\hline 18 & & 27.8 & & & & 3.0 & & \\
\hline 20 & & 11.7 & & & & 2.6 & & \\
\hline 21 & 300 & 11.5 & & & & 2.5 & & 2.8 \\
\hline 22 & & 11.7 & & & & 3.1 & & \\
\hline 23 & & 11.6 & & & & 2.6 & & \\
\hline
\end{tabular}

Plaes toos].

- Probably 1neludeg 2Be obcervat1on. 


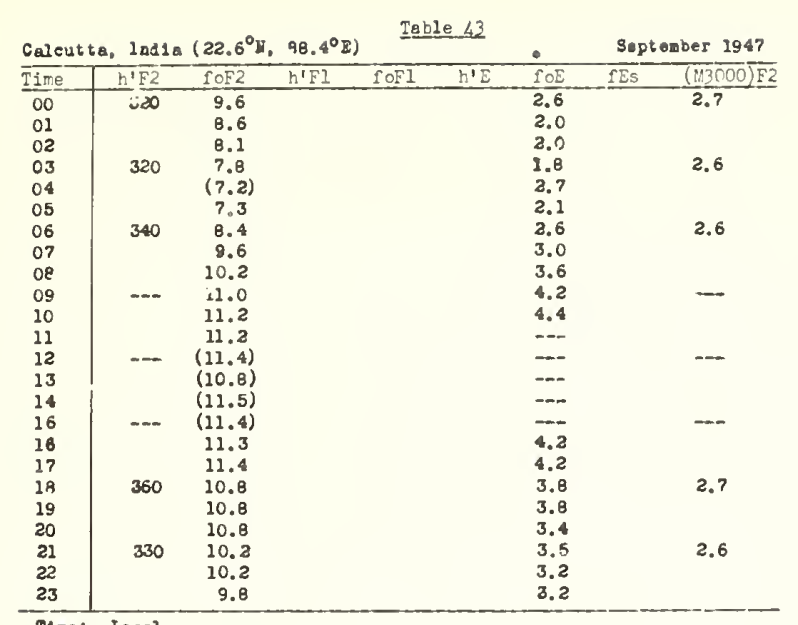

Time! Local.

- Probably includee fBe obeertatione.

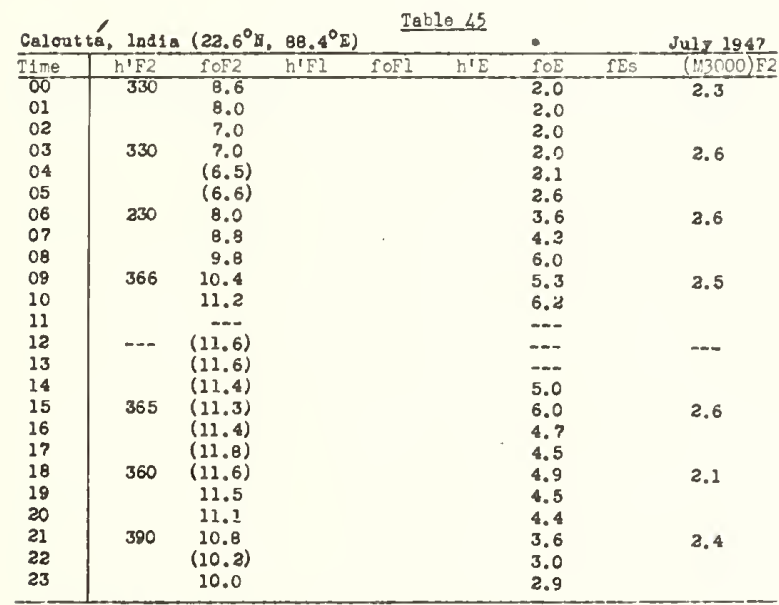

S1me: Looal.

- Probably 1acludes era obserrations.

\begin{tabular}{|c|c|c|c|c|c|c|c|c|}
\hline \multicolumn{2}{|c|}{ Galcutia, } & \multicolumn{4}{|c|}{ Table 47} & \multirow{2}{*}{$\stackrel{-}{\mathrm{fOE}}$} & \multicolumn{2}{|r|}{ May 1947} \\
\hline I1me & $n^{\prime} F 2$ & foF 2 & n'FI & roF 1 & $h^{\prime} E$ & & IES & $(13000) \mathrm{F} 2$ \\
\hline $\begin{array}{l}00 \\
01\end{array}$ & 300 & $\begin{array}{c}11.7 \\
(10.8)\end{array}$ & & & & $\begin{array}{l}1.6 \\
1.2\end{array}$ & & $(2.6)$ \\
\hline 02 & & $(9.8)$ & & & & 1.4 & & \\
\hline 02 & $(300)$ & 8.2 & & & & 1.2 & & $(2.5)$ \\
\hline 04 & & 6.9 & & & & 1.3 & & \\
\hline 05 & & (7.3) & & & & 3.5 & & \\
\hline 06 & $(300)$ & $(9.0)$ & & & & 3.2 & & $(2.6)$ \\
\hline 07 & & $(10,2)$ & & & & 3.6 & & \\
\hline 08 & & $(11.0)$ & & & & 4.0 & & \\
\hline 09 & (345) & $(11.8)$ & & & & 4.8 & & 2.2 \\
\hline 10 & & $(12.0)$ & & & & 6.2 & & \\
\hline 11 & & $(12.4)$ & & & & $\ldots$ & & \\
\hline 12 & 390 & (12.5) & & & & 5.4 & & 2.2 \\
\hline 13 & & $(12.6)$ & & & & $\ldots$ & & \\
\hline 14 & & $(12.0)$ & & & & 5.0 & & \\
\hline 15 & (390) & $(12.0)$ & & & & -- & & $(2,3)$ \\
\hline 16 & & (12.3) & & & & 4.3 & & \\
\hline 17 & & $(12.5)$ & & & & 4.0 & & \\
\hline 18 & $(360)$ & $(12,2)$ & & & & 4.0 & & $(2,3)$ \\
\hline 19 & & $(11.8)$ & & & & -- & & \\
\hline 20 & & (11.6) & & & & 3. 2 & & \\
\hline 21 & 260 & (11.4) & & & & 2.9 & & $(2,4)$ \\
\hline 22 & & $(11.8)$ & & & & 2.1 & & \\
\hline 23 & & (11.6) & & & & 2.0 & & \\
\hline
\end{tabular}

- Frobably iacludeo tEo obeerratioas.

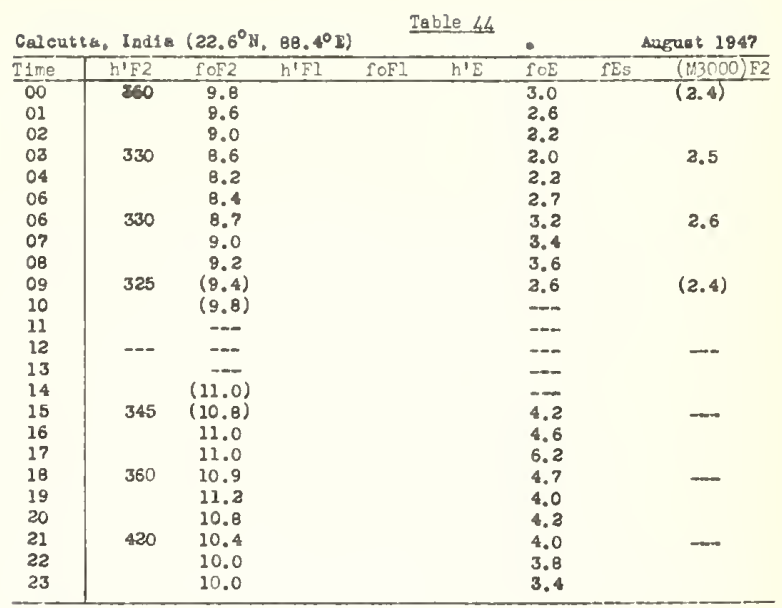

Time: Locar.

- Probubly 1acluden PS obeervationo.

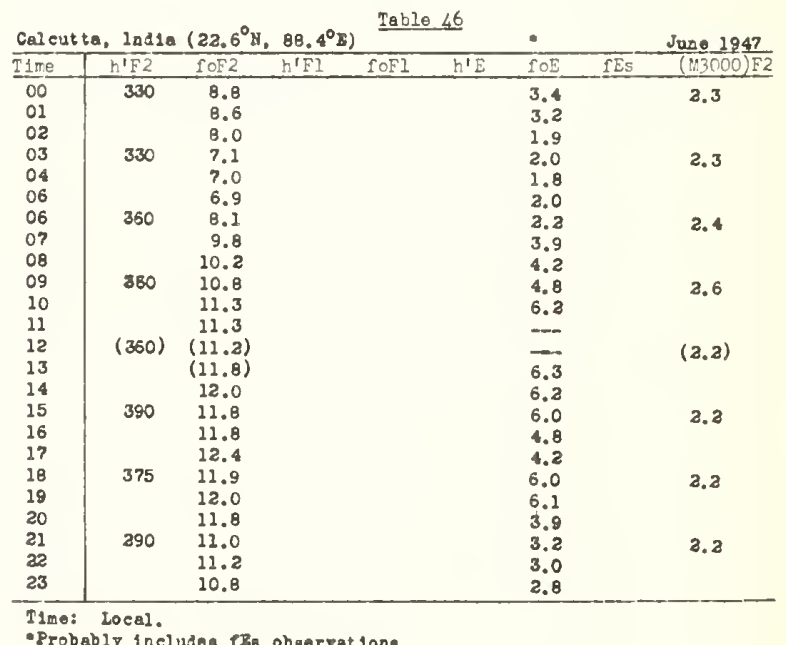

- Probably 1nelude RB obeervatione.

\begin{tabular}{|c|c|c|c|c|c|c|c|}
\hline \multirow{2}{*}{$\frac{\text { Calcut }}{\text { Tlme }}$} & \multicolumn{5}{|c|}{ India $\left(23.6^{\circ} \mathrm{N}\right.$} & & Apr11 1947 \\
\hline & $h^{\prime} F^{2}$ & foF2 & $h^{\prime} E l$ & foFl & $h^{\prime} E$ & $\hat{S O E}$ & $(13000) \overline{\mathrm{F} 2}$ \\
\hline 00 & $(330)$ & $(13.2)$ & & & & $\ldots$ & $(2.6)$ \\
\hline 01 & & $(12.0)$ & & & & 1.6 & \\
\hline 02 & & $(9.0)$ & & & & 1.6 & \\
\hline 03 & --- & $(8.2)$ & & & & $\cdots$ & - \\
\hline 04 & & $(7.6)$ & & & & -- & \\
\hline 05 & & $(7.4)$ & & & & 1.8 & \\
\hline 06 & $(270)$ & $(8.4)$ & & & & 2.6 & $(2.9)$ \\
\hline 07 & & $-\infty$ & & & & 3.0 & \\
\hline $0 \theta$ & & -- & & & & 4.0 & \\
\hline 09 & $(300)$ & $(14,0)$ & & & & 4.6 & $(2,2)$ \\
\hline 10 & & $-\infty-$ & & & & $\ldots$ & \\
\hline 11 & & $(13.2)$ & & & & -- & \\
\hline 12 & 390 & 13.0 & & & & 6.1 & 2.4 \\
\hline 13 & & 13.6 & & & & 6.1 & \\
\hline 14 & & 12,4 & & & & -- & \\
\hline 15 & 360 & $(14.0)$ & & & & 4.4 & $(2.4)$ \\
\hline 16 & & $(12.9)$ & & & & 4.0 & \\
\hline 17 & & $(13.9)$ & & & & - & \\
\hline 18 & $(330)$ & $(14.0)$ & & & & 3.0 & $(2.4)$ \\
\hline 19 & & $(14,3)$ & & & & 2.8 & \\
\hline 20 & & $(14,3)$ & & & & 2.6 & \\
\hline 21 & $(230)$ & $(14.1)$ & & & & 2.2 & $(3.4)$ \\
\hline 23 & & $(14.4)$ & & & & - & \\
\hline 23 & & $(14.2)$ & & & & $\cdots$ & \\
\hline
\end{tabular}

- Probably includer ren obsertatione. 
18

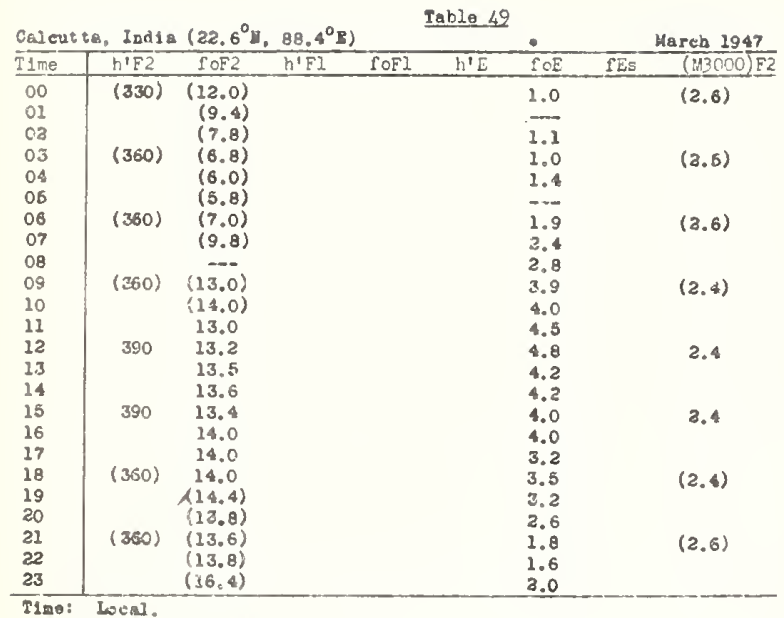

- Probably incladae eBe obeorvationa.

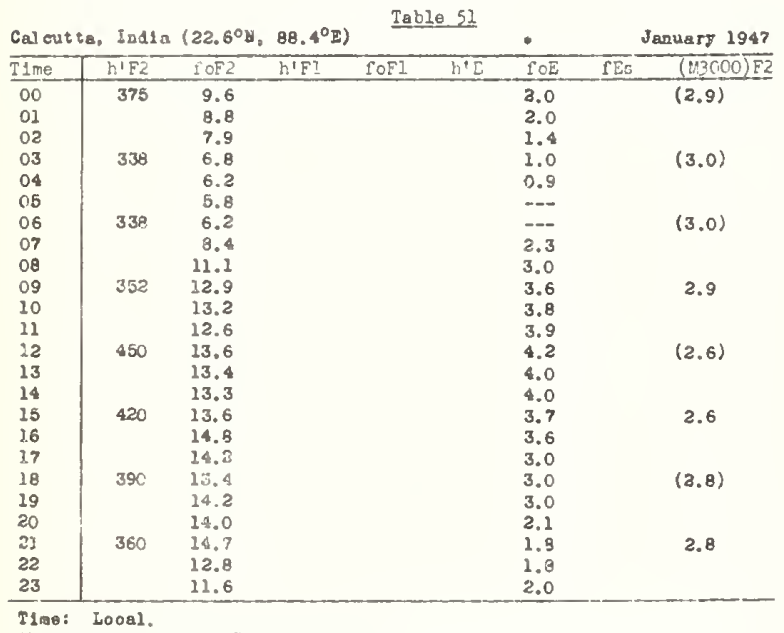

Probably contalos Ps oboarvations.

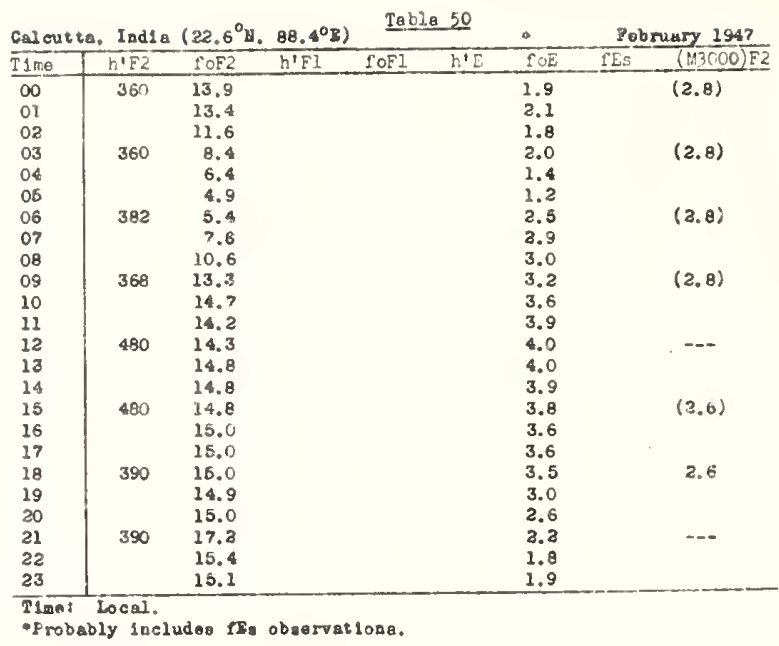




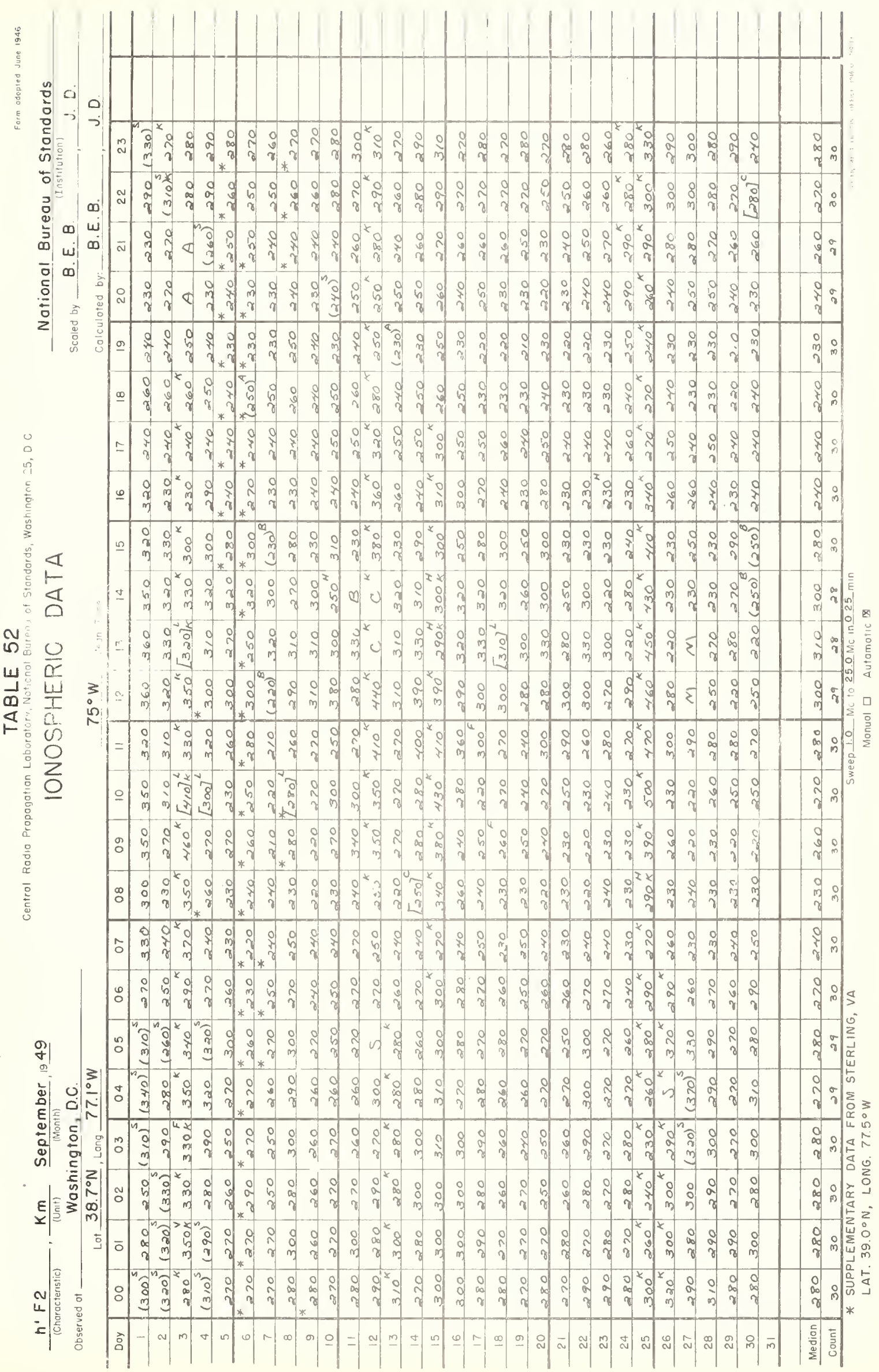




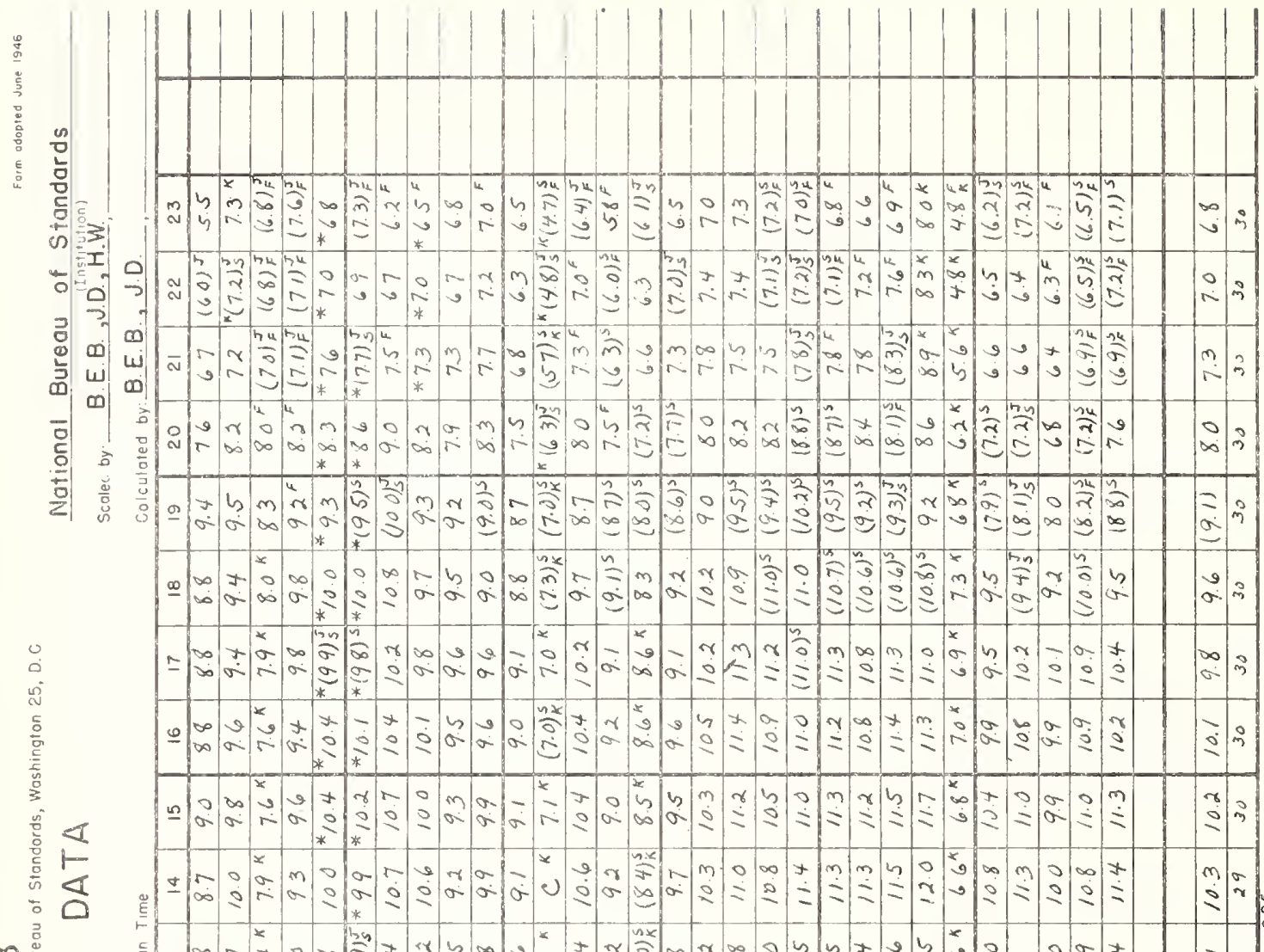

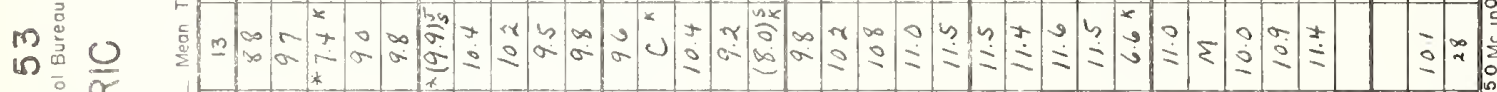

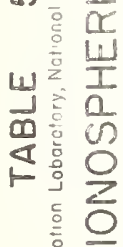

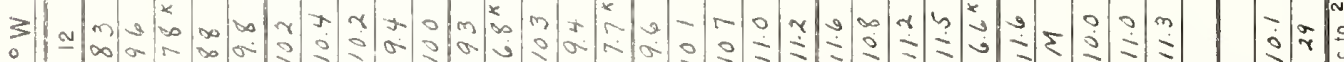

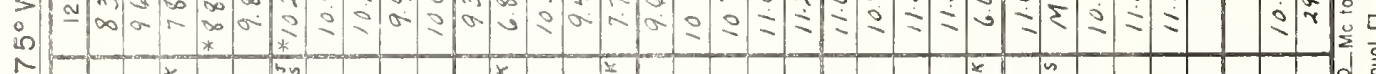

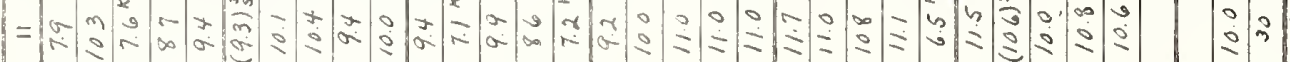

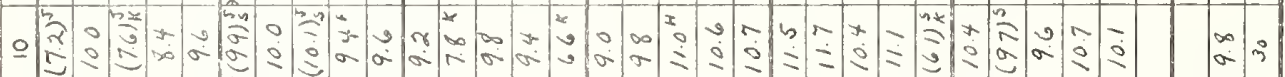

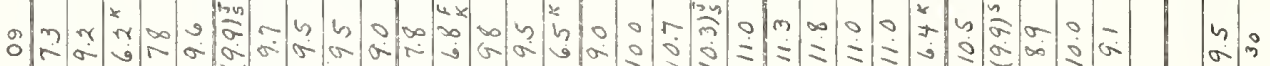
5

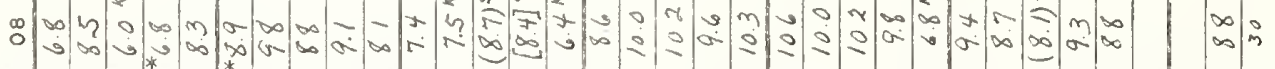

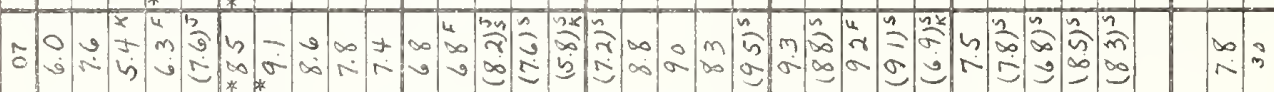

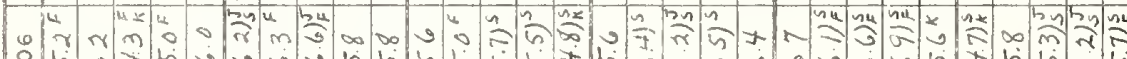

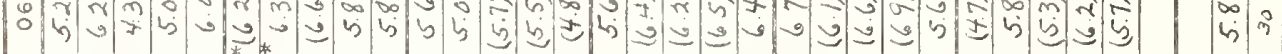

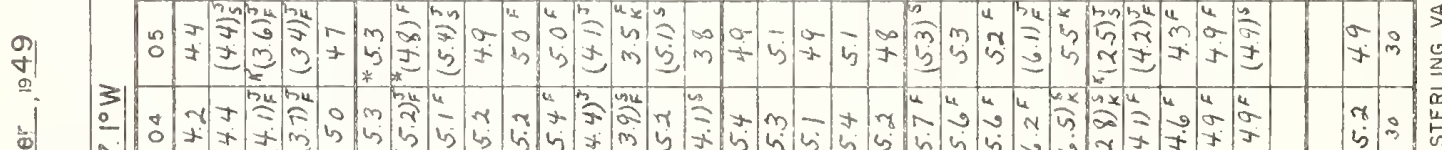

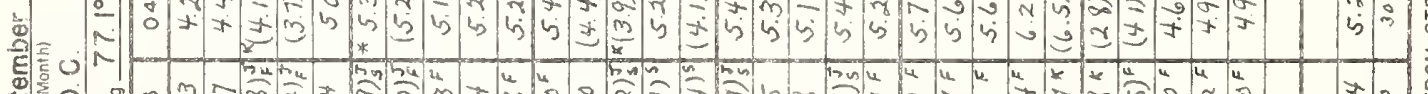

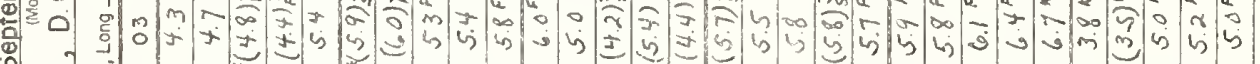

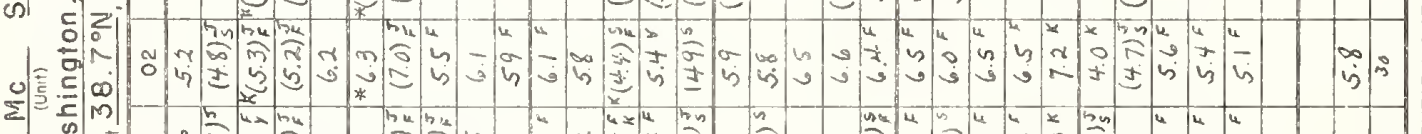

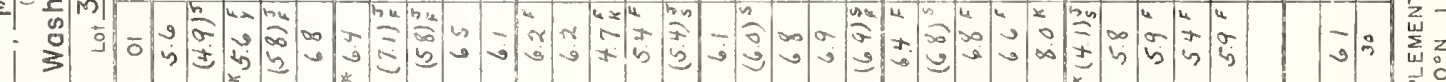

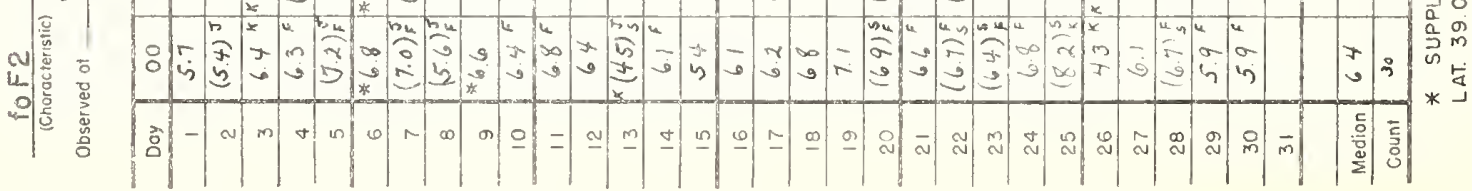




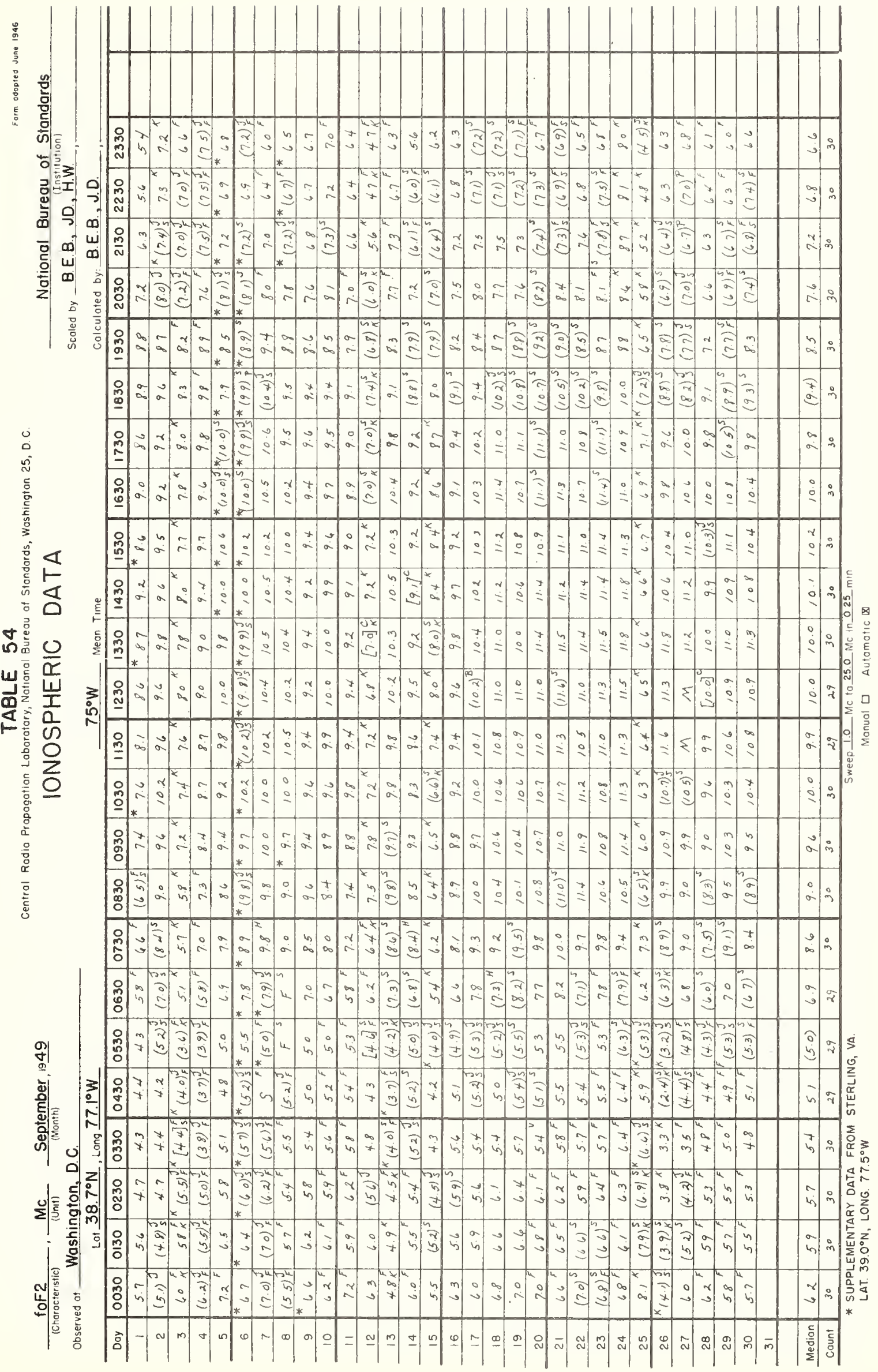


인

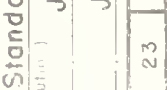

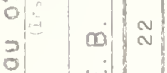

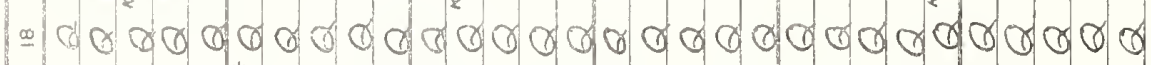

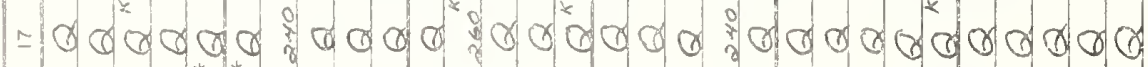

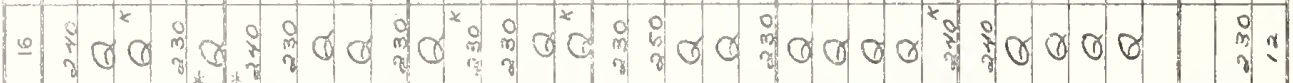

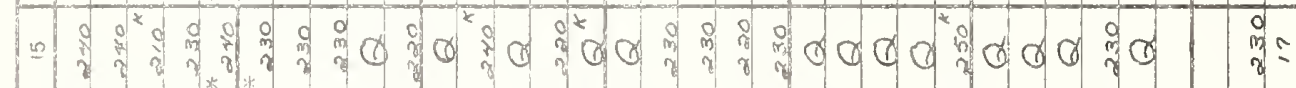

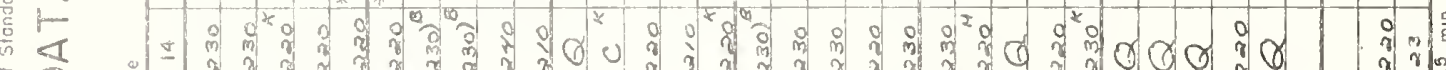

经 E $ว x$ म

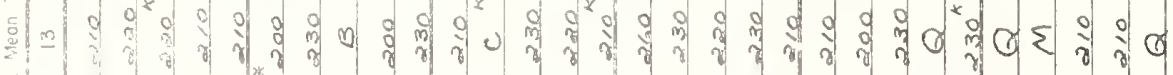
3 둔 in:

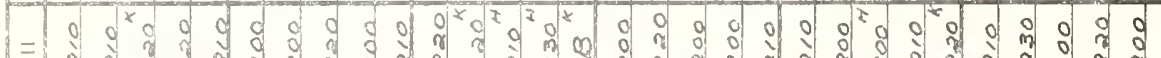

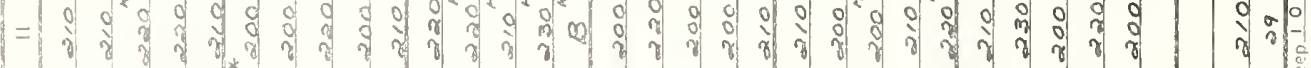

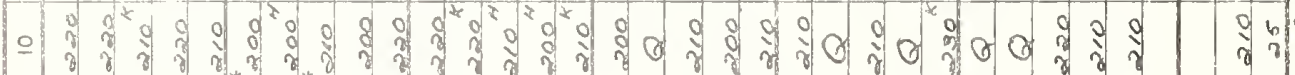

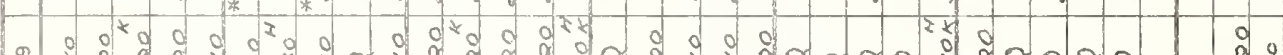

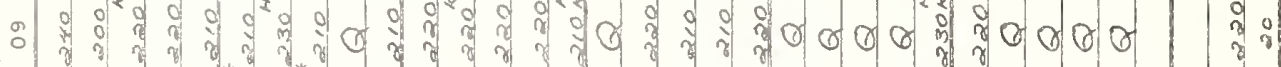

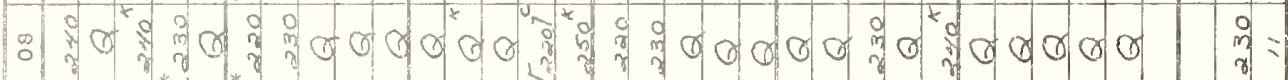

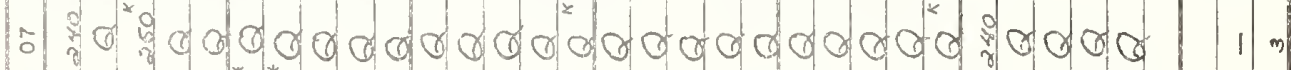

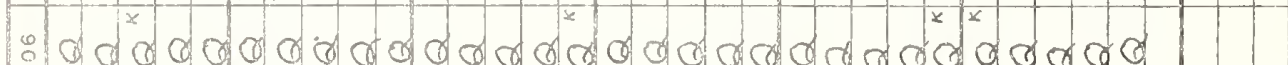
ragagerag

\&

잉

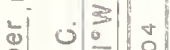

हो

है 


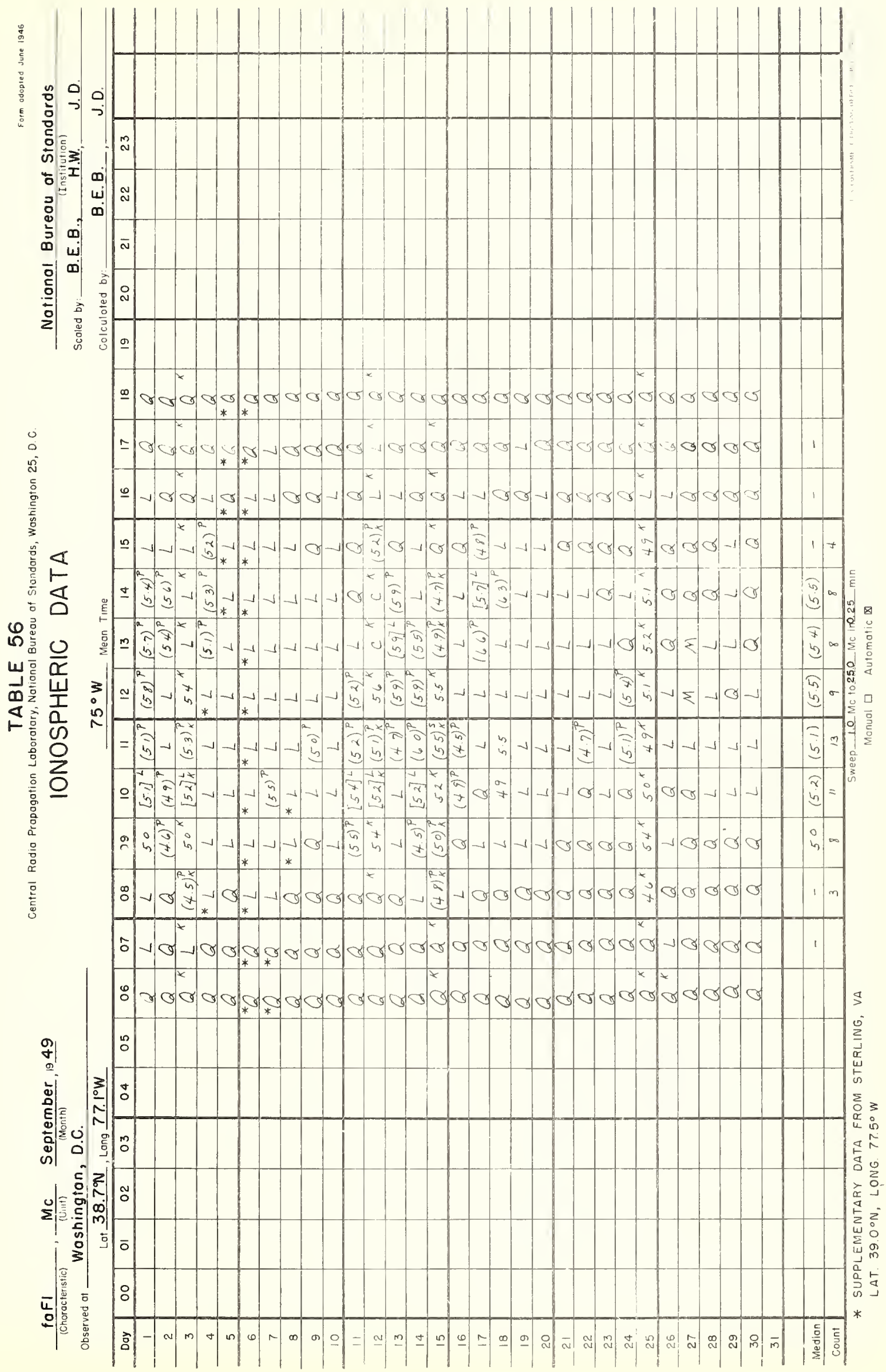




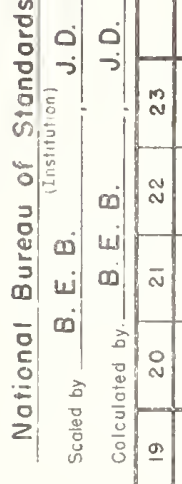

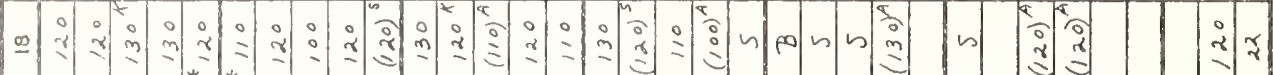

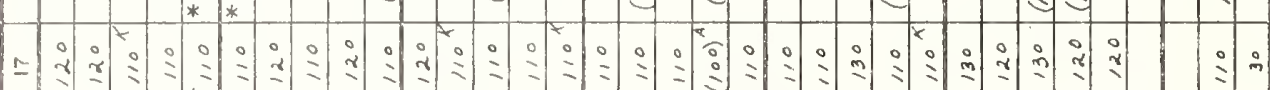

要

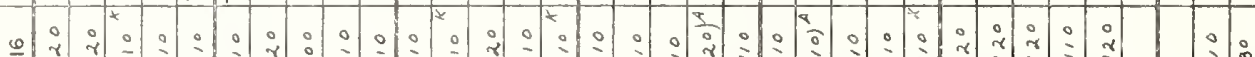

핳

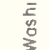

응

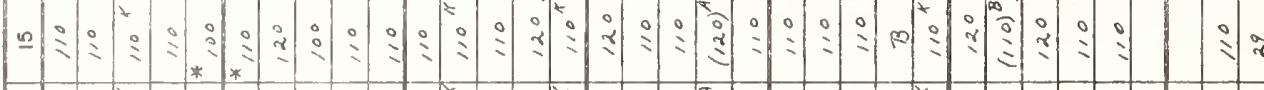

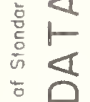

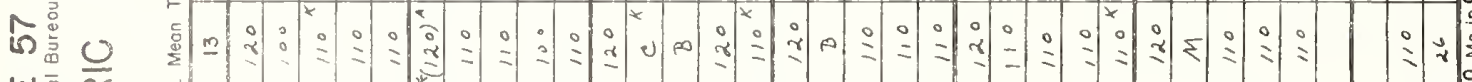

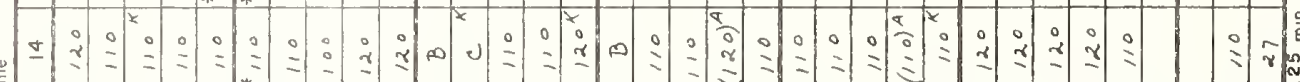

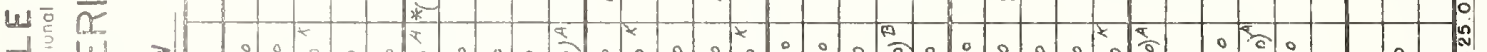

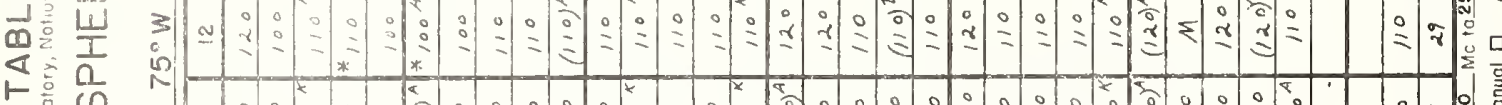

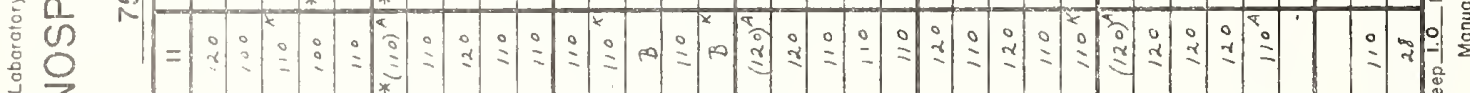

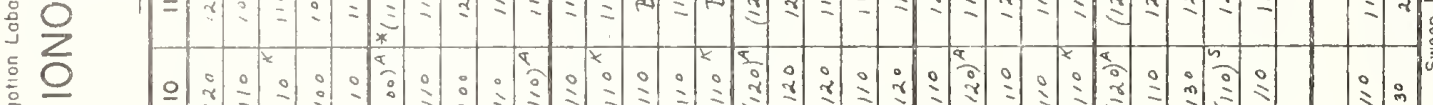

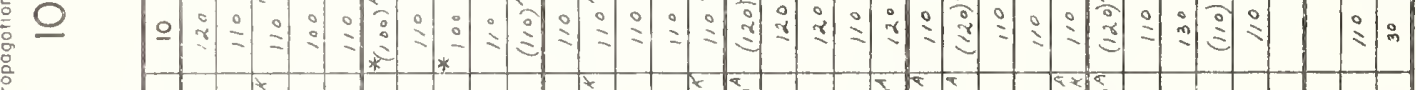

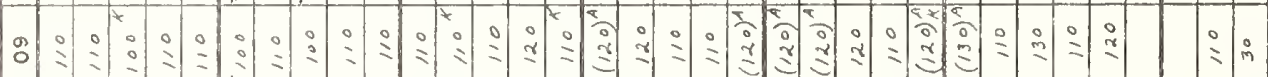

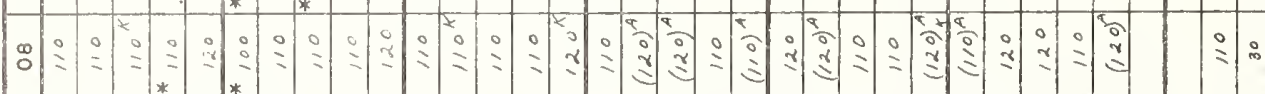

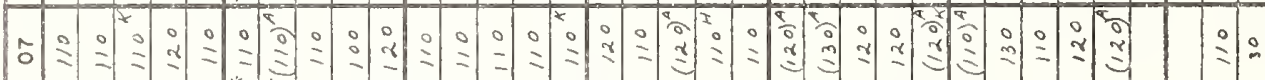

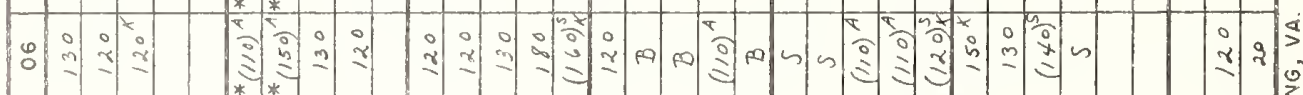

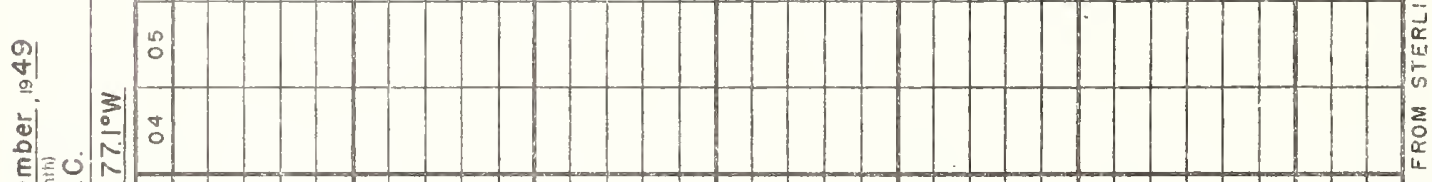

岁

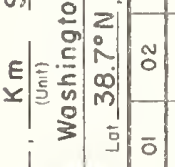

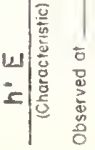

응

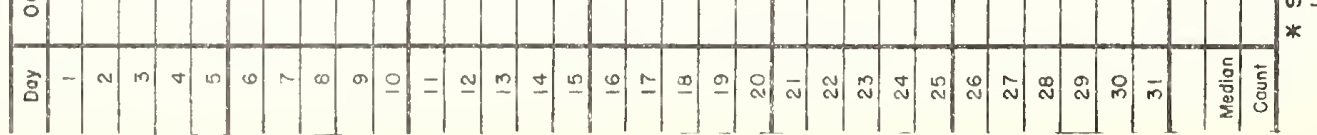




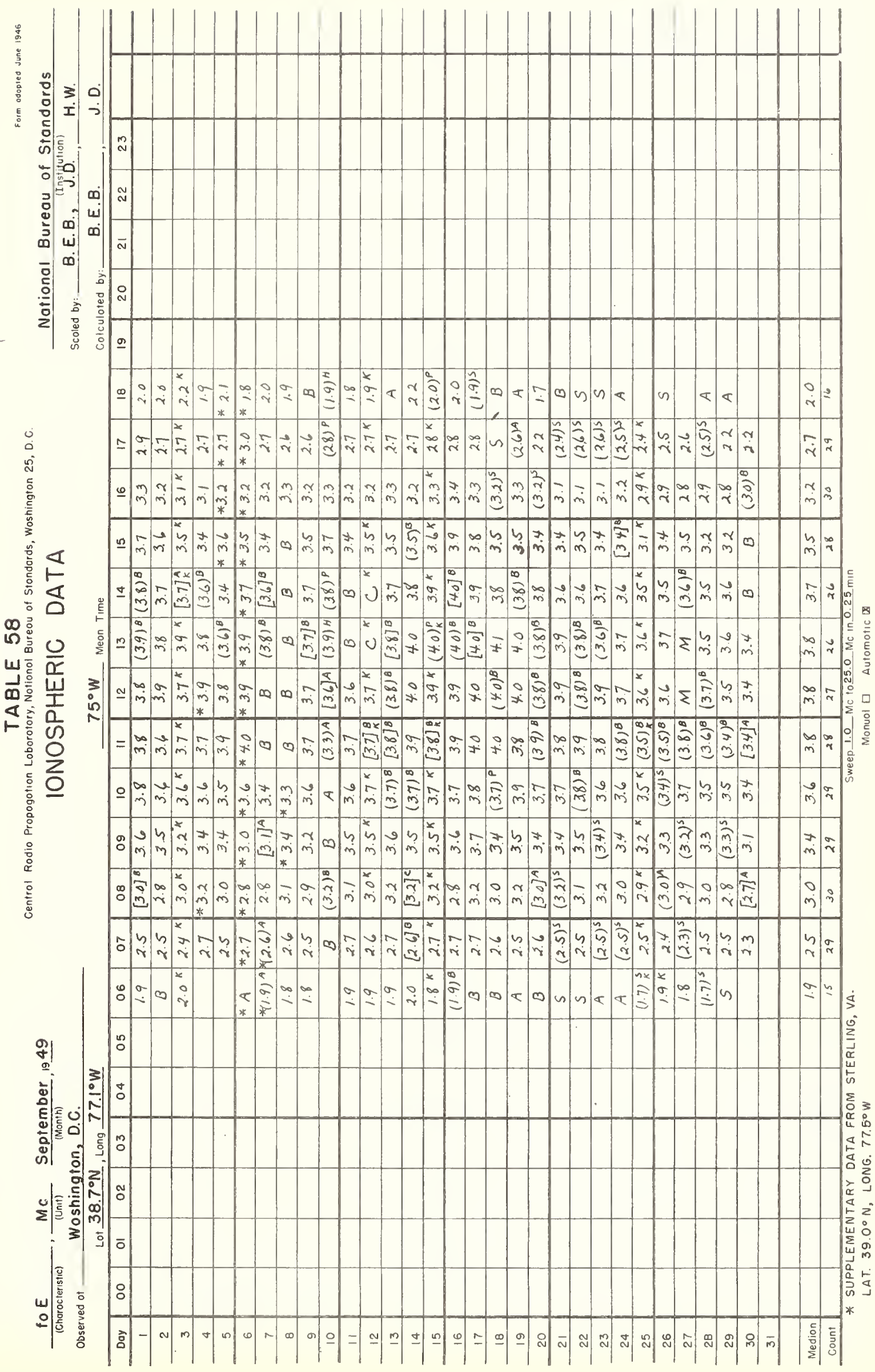




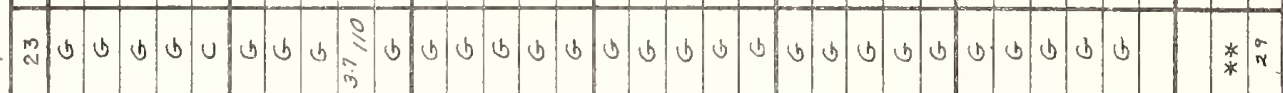

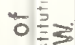

일

$\frac{\sqrt{3}}{4}$

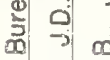

동 की

เ.

$\begin{array}{lllllll}0 & b & b & b & u\end{array}$

(1) 4.

ฟै.

$৬ ৬$

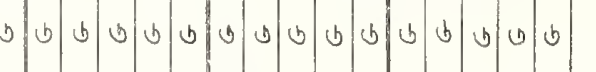

*

…

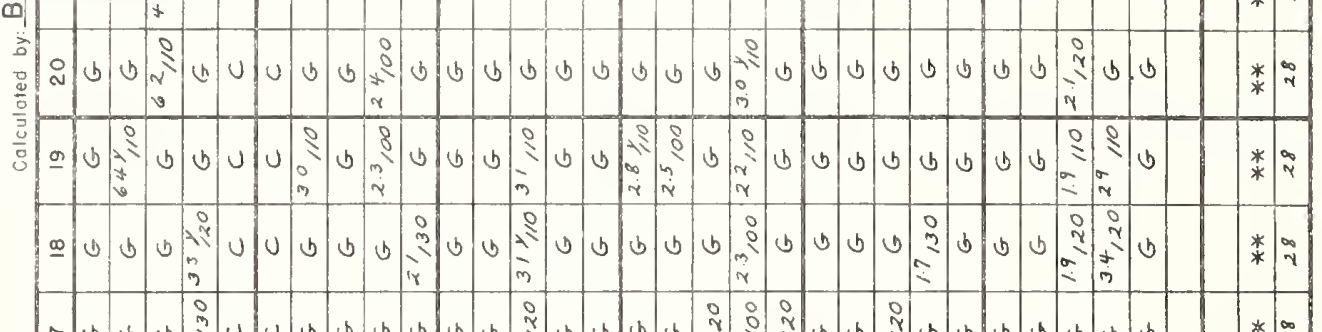

$\dot{0}$

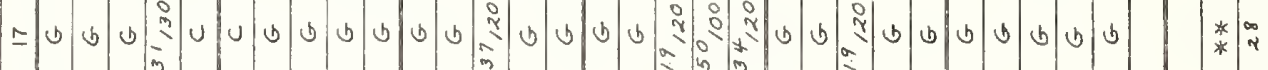

ลั

○

$\frac{g^{\prime}}{5}$

(5)

空

喜

i

"

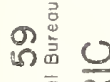

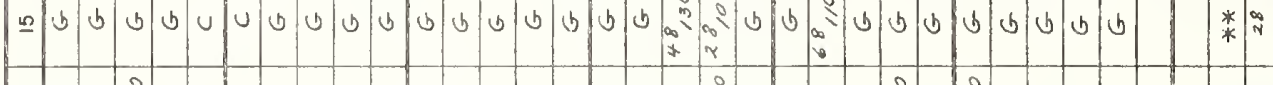

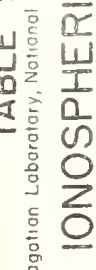

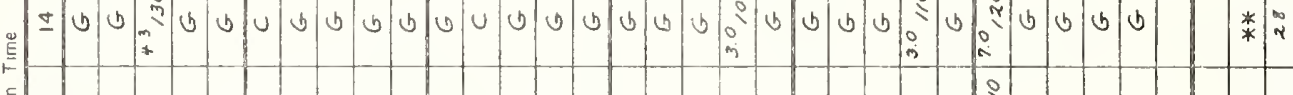

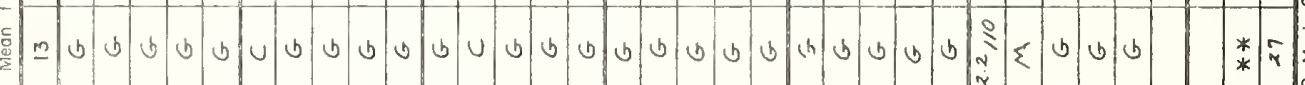

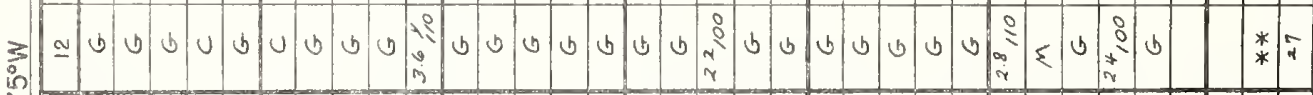

in

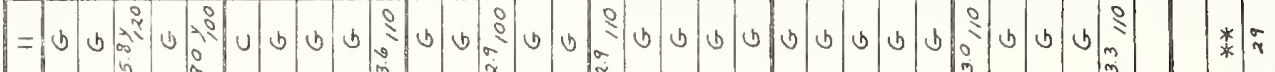

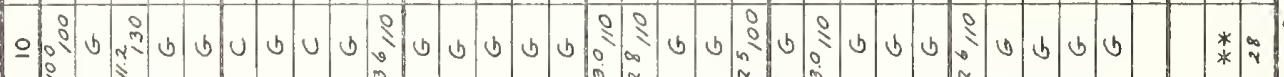

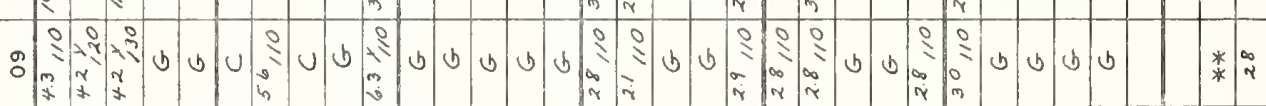

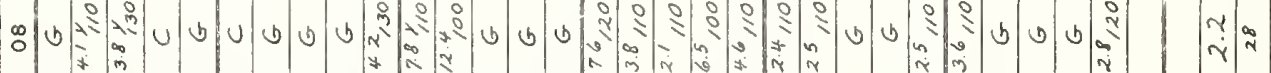

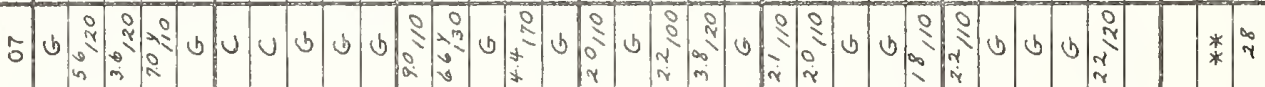

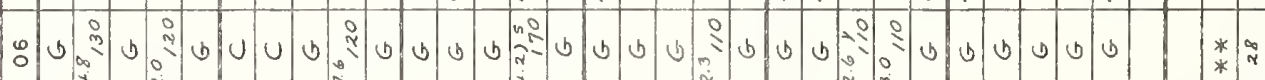

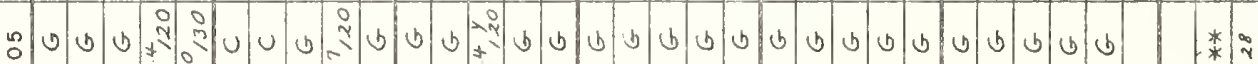

古

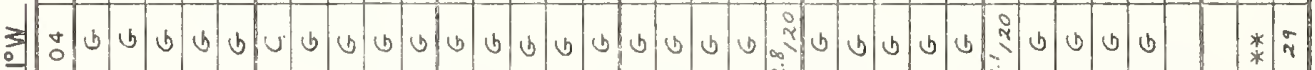

N.

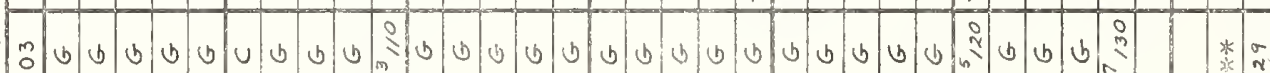

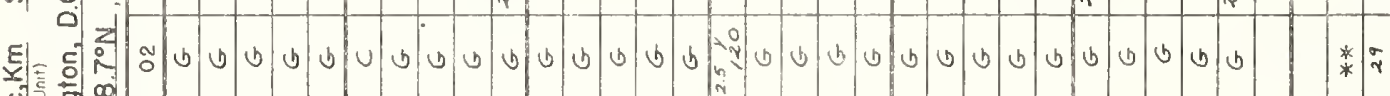

$\stackrel{0}{\Sigma}$

公

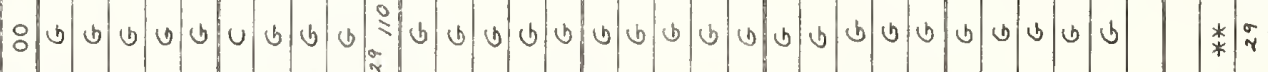

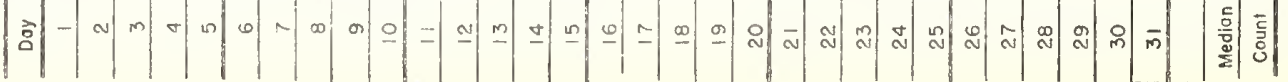




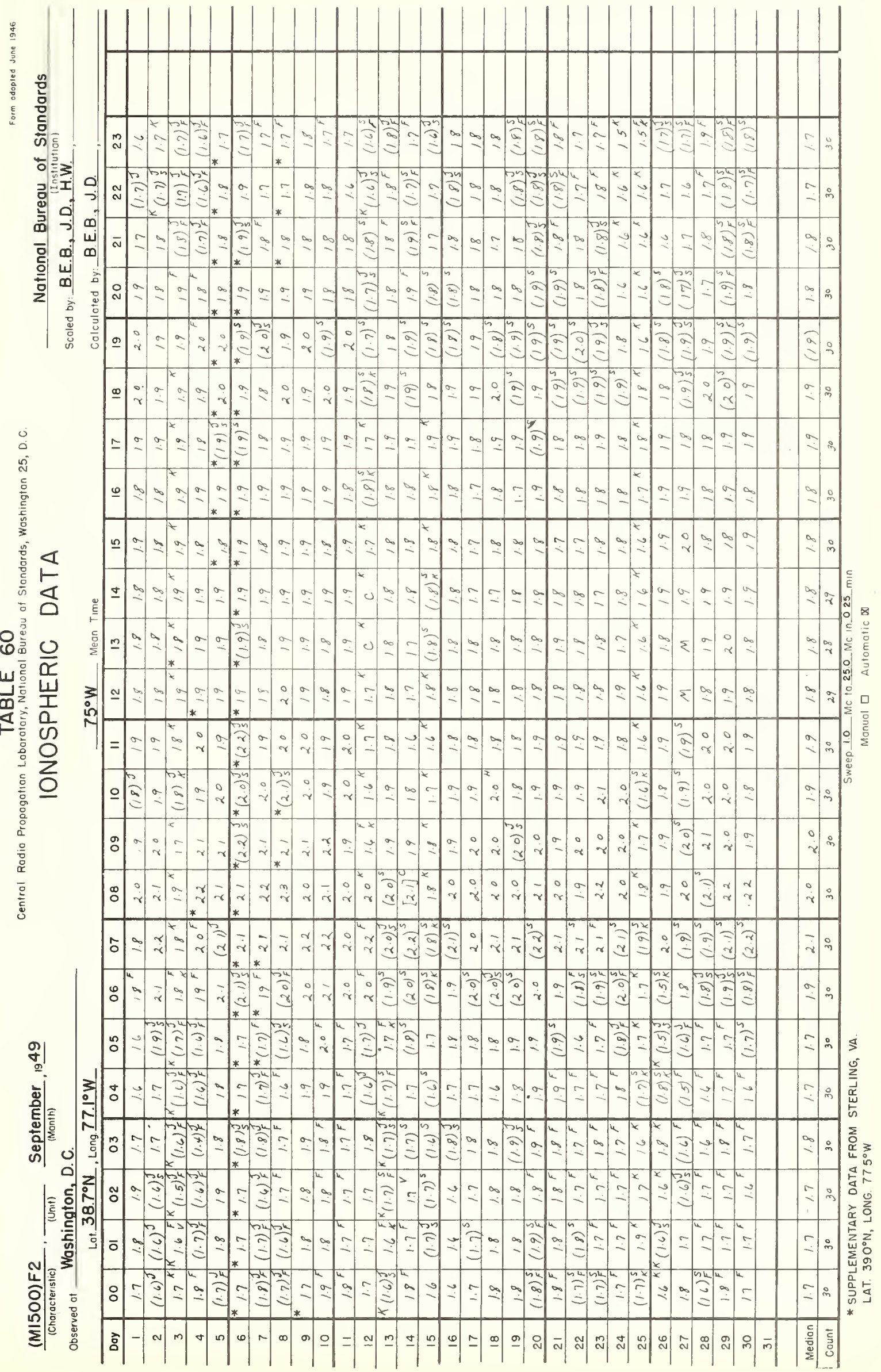




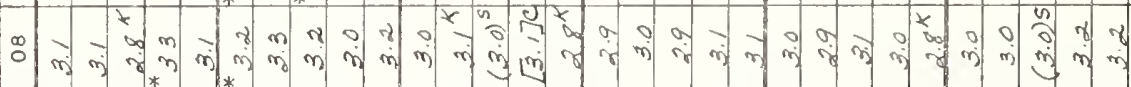

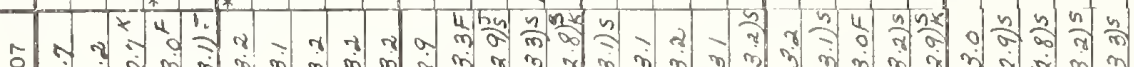

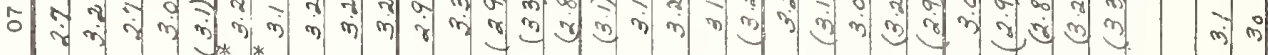

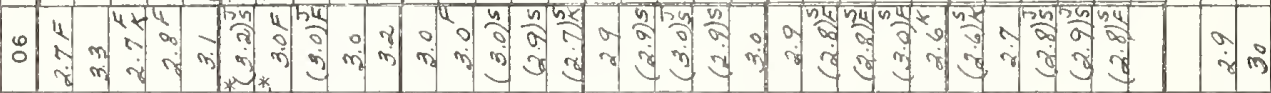

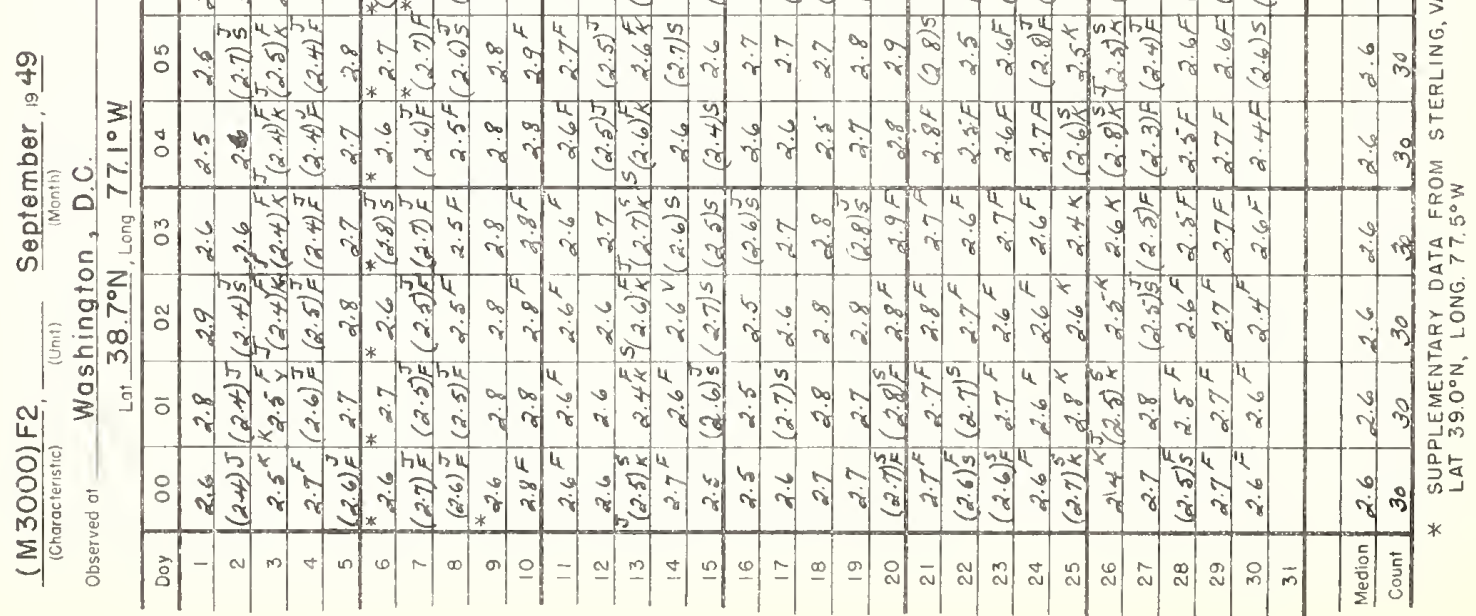




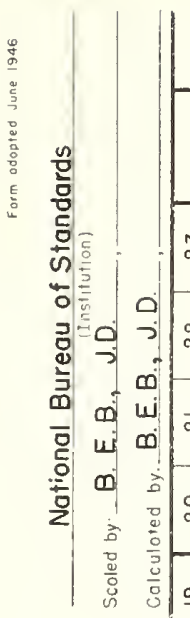

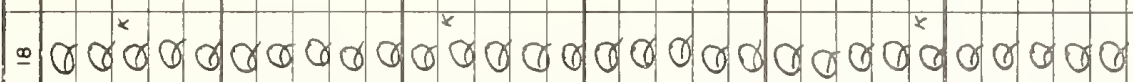
$=$ d d d

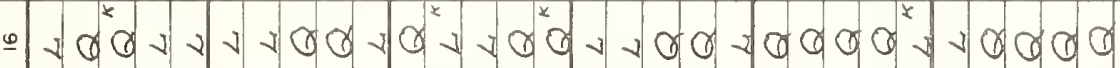

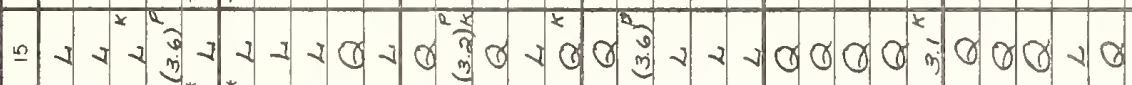

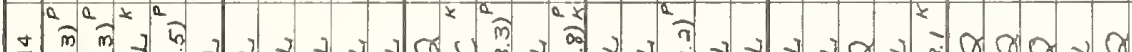

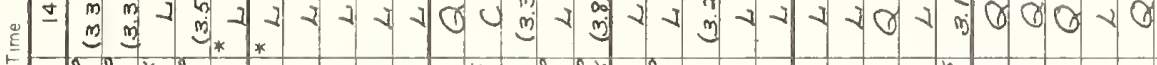
है।

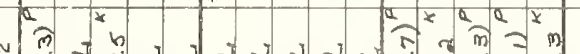

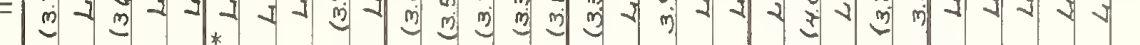

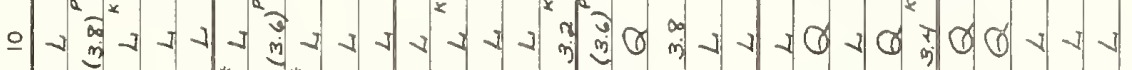

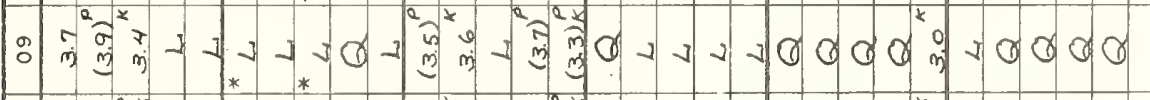

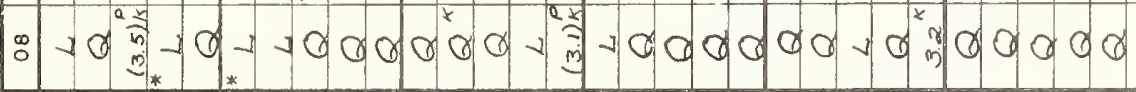
$\therefore \alpha^{2}$ -

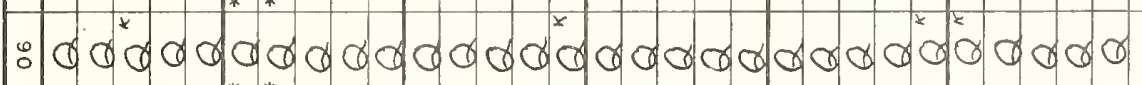

का ๑

కุo

हो

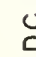
:

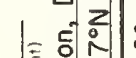


문

\section{ว}

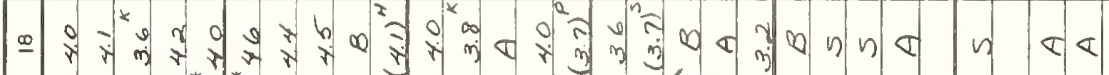

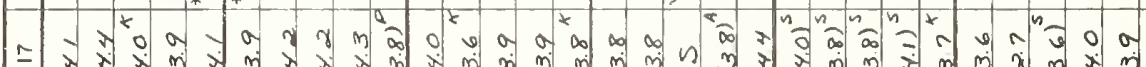
二

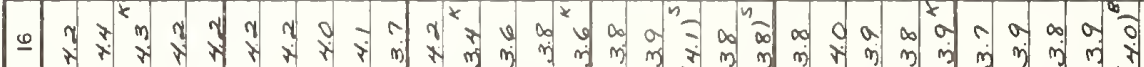

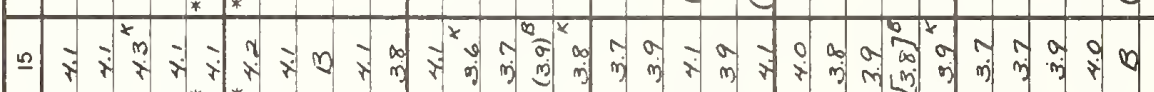

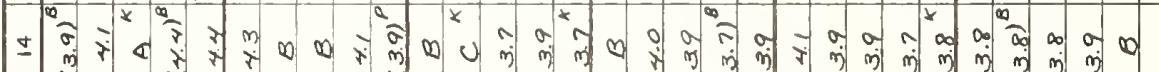

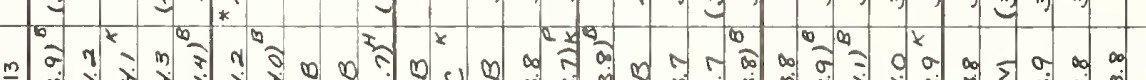

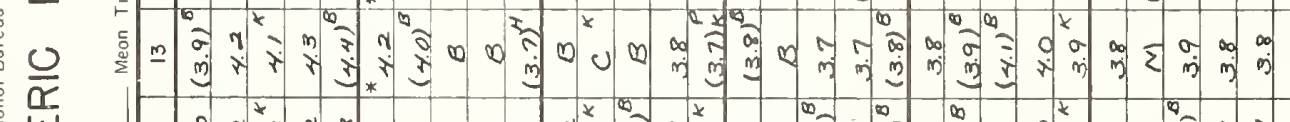

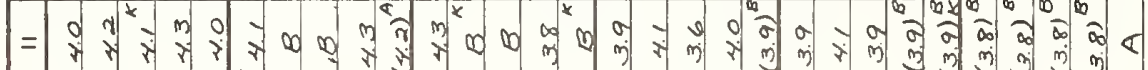

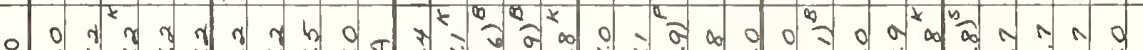

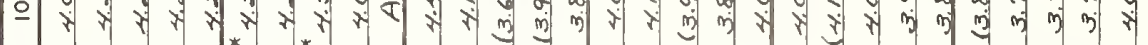

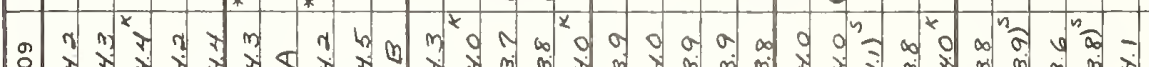

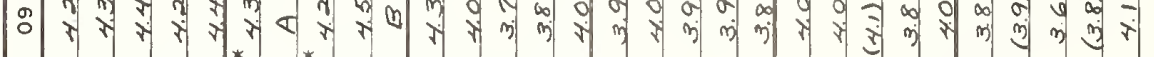

$+\infty$

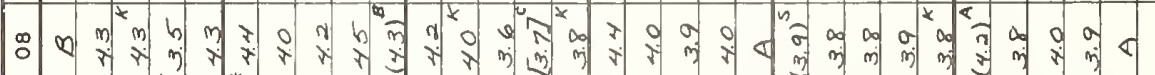

จํ.

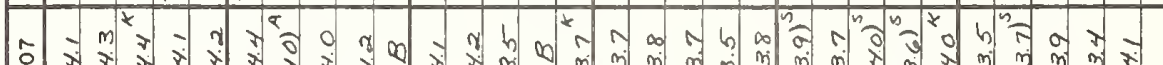

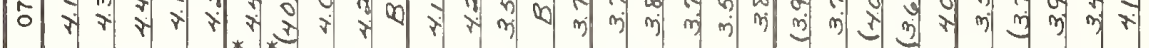

$\left.\therefore 00\right|^{x}=7$ बैंतें

शे| \%

象

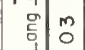

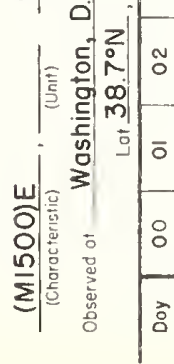

$\overline{0}$

: 
Table 64

Ionospheric Storminess at Jashington, D. C.

September 1942

\begin{tabular}{|c|c|c|c|c|c|c|}
\hline Day & $\begin{array}{l}\text { Ionospheric } \\
00-12 G C T\end{array}$ & $\begin{array}{l}\text { charactrer* } \\
12-2 d_{4} \text { GCI }\end{array}$ & $\begin{array}{l}\text { Principal } \\
\text { Beginning } \\
\text { GCT }\end{array}$ & $\begin{array}{l}\text { storms } \\
\text { Ind } \\
\text { GCT }\end{array}$ & $\begin{array}{l}\text { Geomagneti } \\
00-12 \mathrm{GCT}\end{array}$ & $\begin{array}{l}\text { character* } \\
12-24 \mathrm{GCI}\end{array}$ \\
\hline 1 & 2 & 3 & & & 2 & 3 \\
\hline 2 & 3 & 1 & 2200 & $\ldots$ & 3 & 3 \\
\hline 3 & 4 & 4 & $\cdots$ & 1900 & 5 & 3 \\
\hline 4 & 2 & 2 & & & 3 & 2 \\
\hline 5 & $I$ & 0 & & & 2 & 2 \\
\hline 6 & $I$ & 1 & & & 3 & $I$ \\
\hline 7 & $I$ & $I$ & & & 2 & 1 \\
\hline 8 & 2 & 0 & & & 2 & 3 \\
\hline 9 & 1 & 2 & & & 2 & 0 \\
\hline 10 & 1 & 2 & & & 0 & 2 \\
\hline 11 & 1 & 2 & & & 3 & 2 \\
\hline 12 & 2 & 6 & 0800 & $\cdots$ & 3 & 3 \\
\hline 13 & 4 & 2 & $\cdots$ & 0600 & 3 & 2 \\
\hline 14 & 3 & 3 & & & 2 & 3 \\
\hline 15 & 3 & 4 & 0600 & 1800 & 2 & 2 \\
\hline 16 & 3 & 3 & & & 2 & 2 \\
\hline 17 & 2 & 2 & & & 1 & 3 \\
\hline 18 & $I$ & 2 & & & $I$ & 0 \\
\hline 19 & 1 & 1 & & & 0 & 0 \\
\hline 20 & 1 & 2 & & & 0 & 0 \\
\hline 21 & 1 & 2 & & & 0 & $I$ \\
\hline 22 & 2 & $I$ & & & 2 & 1 \\
\hline 23 & $I$ & $I$ & & & $I$ & $I$ \\
\hline 24 & $I$ & 2 & 2100 & $-\infty-\infty$ & 2 & 3 \\
\hline 25 & 4 & 6 & $\cdots$ & $-\ldots$ & 4 & 3 \\
\hline 26 & 4 & 1 & $\cdots$ & 0700 & 3 & 2 \\
\hline 27 & 2 & $I$ & & & 4 & 3 \\
\hline 28 & 2 & 2 & & & 3 & 1 \\
\hline 29 & $I$ & 2 & & & 2 & 1 \\
\hline 30 & 2 & 1 & & & 3 & 2 \\
\hline
\end{tabular}

* Ionosphere character figure (I-figure) for ionospheric storminess at Tashington, D. C., during 12-hour period, on an arbitrary scale of 0 to 9,9 representing the greatest disturbance.

**Average for 12 hours of Cheltenham, haryland, geomagnetic K-figures on an arbitrary scale of 0 to 9,9 representing the greatest disturbance. -..-Dashes indicate continuing storm. 


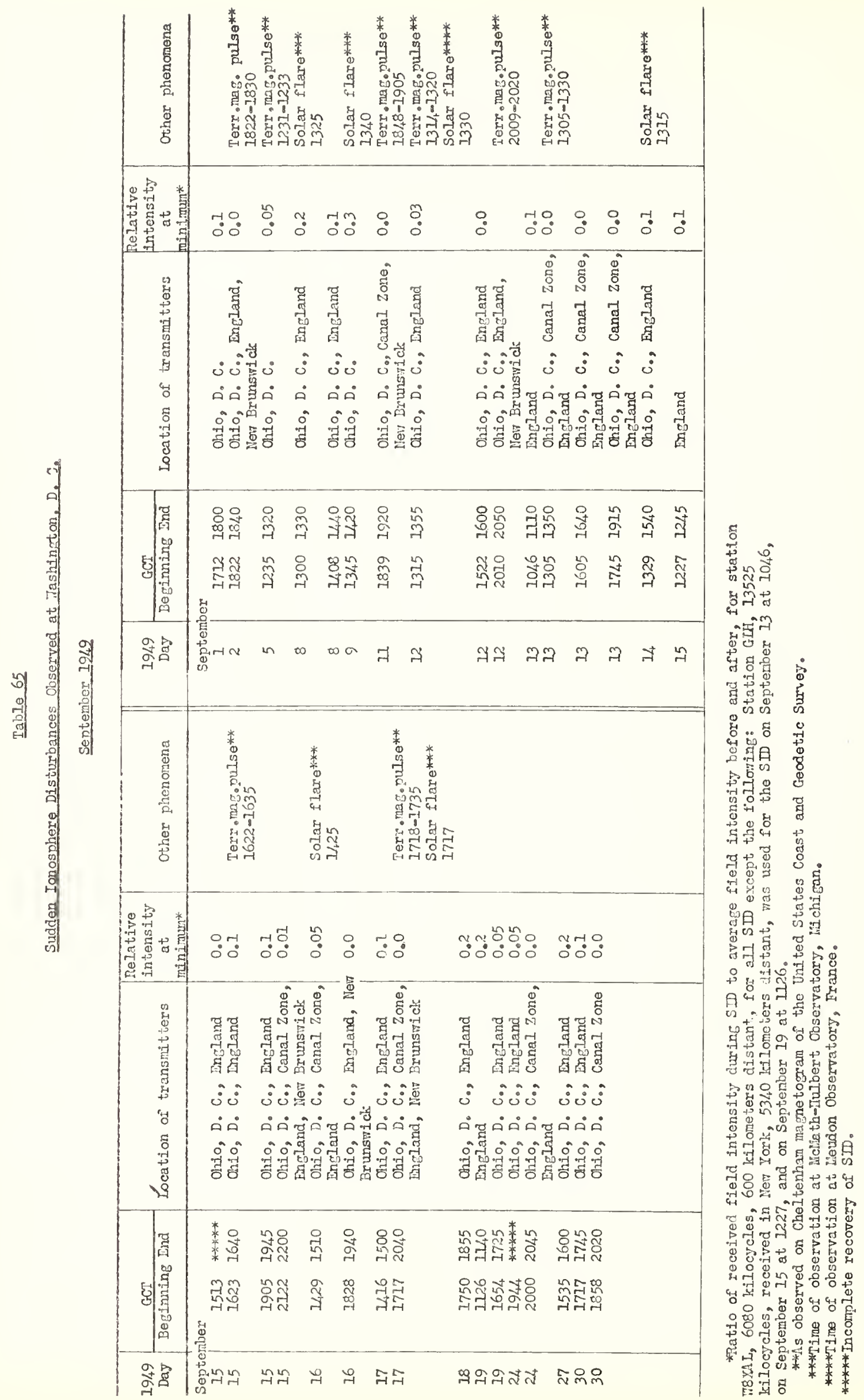


Table 66

Sudden Ionos phere Disturhances Renorted by Engineer-in-Chief,

Cable and Wireless, Ltd. as Observed in England

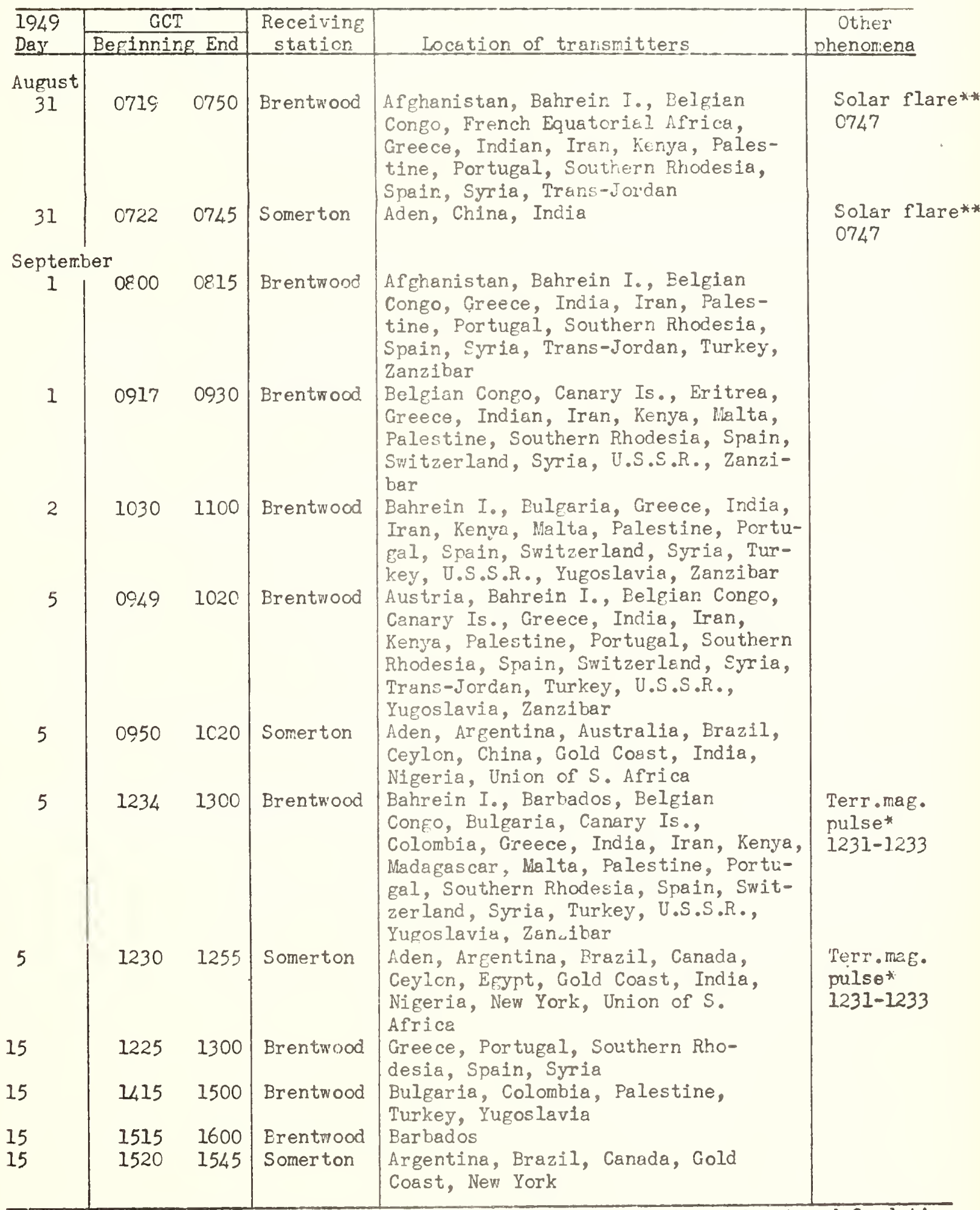

*As observed on Cheltenham magnetogram of the United States Coast and Geodetic 
Table 67

Sudden Ionosphere Disturbances Reported by International Telephone and Telegraph Corporation, as Observed at Platanos. Argentina

\begin{tabular}{|c|c|c|}
\hline $\begin{array}{l}1949 \\
\text { Day }\end{array}$ & $\frac{\text { GCT }}{\text { Beginning Find }}$ & Iocatjon of transmitters \\
\hline August & $1120 \quad u_{r} 00$ & Belgium, Cermany, Netherlands, New Yori, Venezuela \\
\hline
\end{tabular}

Table 68

Sudden Ionosphere Disturbances Reported by RCA Commuications, Inces as Observed at Point Reyes California

\begin{tabular}{|c|c|c|c|c|}
\hline $\begin{array}{l}1949 \\
\text { Day }\end{array}$ & \multicolumn{2}{|l|}{ GCT } & Location of transmitters & $\begin{array}{l}\text { Other } \\
\text { phonomene }\end{array}$ \\
\hline $\begin{array}{l}\text { September } \\
\text { II }\end{array}$ & 1850 & 1910 & Australia, Haraii, Japan, Philippine Is. & $\begin{array}{l}\text { Terr.mag. } \\
\text { pulse* } \\
1848=1905\end{array}$ \\
\hline 12 & 0630 & 0700 & China, Japan, Philinpine Is. & $\begin{array}{l}\text { Solar flare } \\
0620\end{array}$ \\
\hline 13 & 0353 & 0430 & $\begin{array}{l}\text { Australia, China, Japan, Java, Philippine } \\
\text { Is. }\end{array}$ & \\
\hline 15 & 2126 & $2 I 50$ & $\begin{array}{l}\text { Australia, China, Hawaí, Japan, Java, } \\
\text { New York, Philippine Is. }\end{array}$ & \\
\hline 17 & 1720 & 1900 & Australia, China, Harraii, Japar & $\begin{array}{l}\text { Terr.mag. } \\
\text { pulse* } \\
1718-1735 \\
\text { Solar flare*** } \\
1717\end{array}$ \\
\hline 18 & 0215 & 0230 & $\begin{array}{l}\text { Australia, China, Chosen, Hawail, Japan, } \\
\text { Java, Philippine Is. }\end{array}$ & \\
\hline $\begin{array}{l}\text { October } \\
\quad 2\end{array}$ & 0322 & 0400 & Australia, China, Japan, Philippine Is. & \\
\hline
\end{tabular}

* As observed on Cheltenham magnetogram of the United States Coast and Geodetic Survey。

* Iime of observation at Prague Observatory, Czechos lovakia. ***Ime of observation at licklath-liulbert Observatory, lif chigan. 
Table 69

Sudden Ionosphere Disturbances Reported by RCA Communications. Inces

as Cbserved at Riverhead, New York

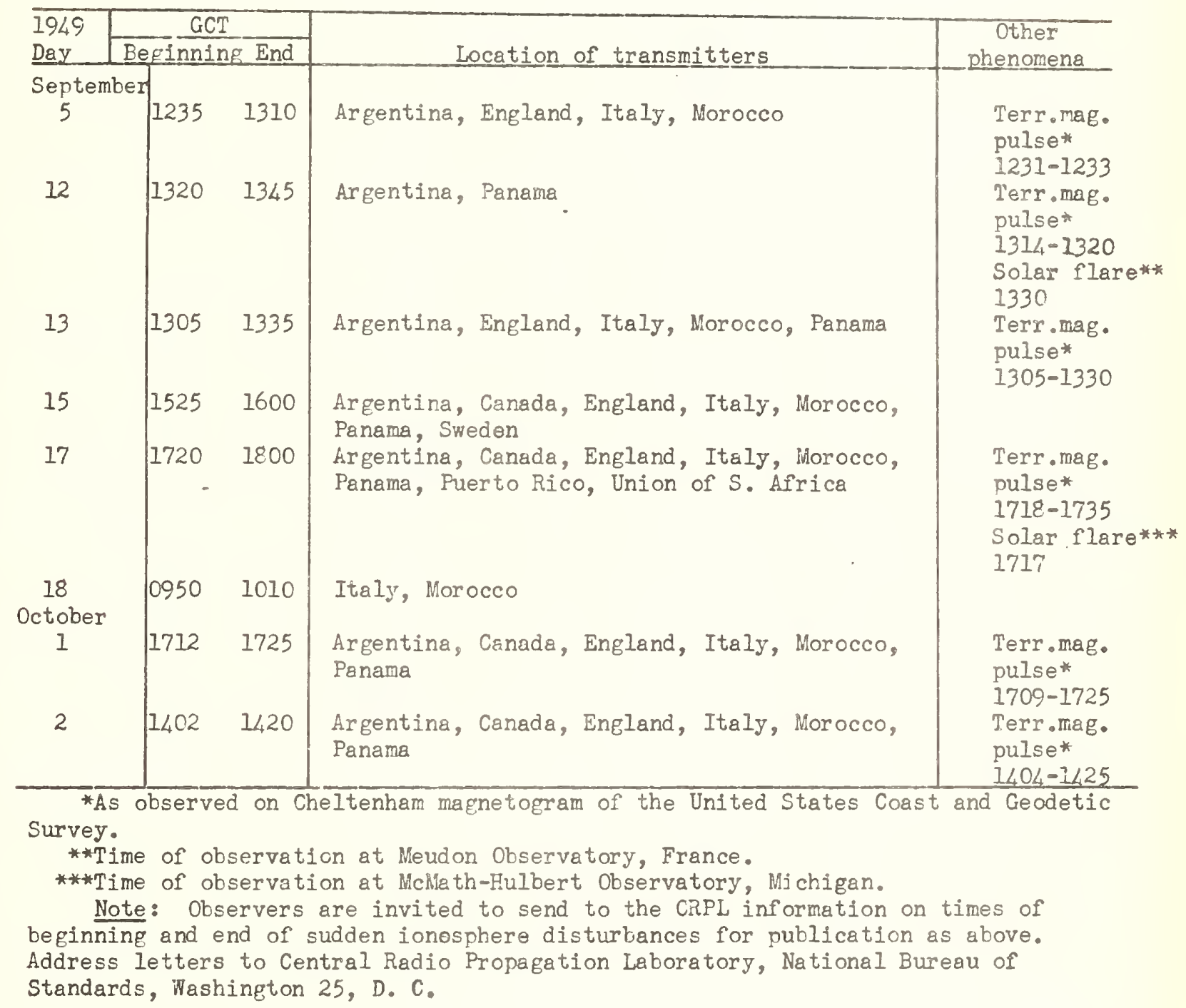


Frovisional Radio Propagation Mality Figure日

(Including Comparison with CRPL Waraings and CRPL Probable Disturbod PBriod Forecasts) 늘 1949

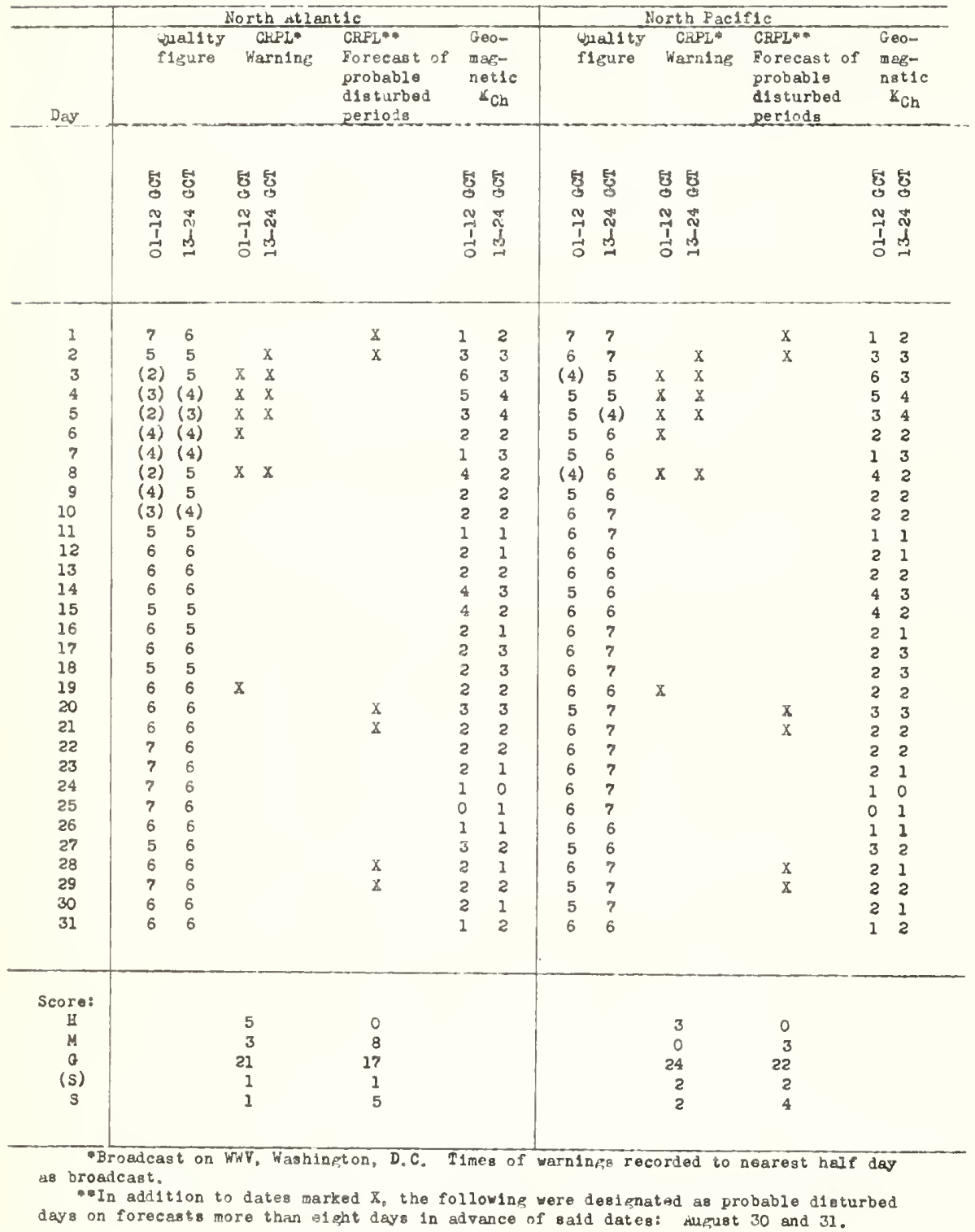

Sal1ty F1gure Scale: 1 - Useles

2 - Very poor 3 - Foor

4 - Poor to falr

5 - Falr

6 - Felr to good

7 - Good

8 - Very gooz

9 - Excellent
Symbols:

4 Warning given or probablo dlaturbed date

ㅂ wallty 4 or worss on day or half day of warning

$M$ unallty 4 or worse on day or half dey of no warning

Q Gality 5 or better on day of no warn$1 \mathrm{gB}$

(s) Dallty 5 on dey of varning

5 Oualty 6 or better on day of warning

( ) Jual 1ty 4 or worso (disturbed)

Goomagnetic $\mathrm{KCh}$ on the $s$ tandard acale of 0 to 9, 9 representing the greatest disturbance. 
Table 71

American and Zürich Provisional Relative Sunspot Numbers

September 1949

\begin{tabular}{|c|c|c|c|c|c|}
\hline Date & $\mathrm{R}_{\mathrm{A}^{*}}$ & $\mathbb{I}_{Z^{*}}$ & Date & $\mathrm{R}_{\mathrm{A}^{*}}$ & $\mathrm{~B}_{Z^{4}}$ \\
\hline 1 & 234 & 165 & 17 & 216 & 182 \\
\hline 2 & 225 & 165 & 18 & 226 & 210 \\
\hline 3 & 224 & 184 & 19 & 223 & 154 \\
\hline 4 & 229 & 184 & 20 & 221 & 170 \\
\hline 5 & 223 & 174 & 21 & 187 & 130 \\
\hline 6 & 222 & 188 & 22 & 199 & 148 \\
\hline 7 & 212 & 184 & 23 & 175 & 144 \\
\hline 8 & 212 & 170 & 24 & 167 & 127 \\
\hline 9 & 196 & 160 & 25 & 63 & 136 \\
\hline 10 & 220 & 164 & 26 & 138 & 108 \\
\hline 11 & 235 & 176 & 27 & 101 & 94 \\
\hline 12 & 213 & 173 & 28 & 92 & 84 \\
\hline 13 & 200 & 170 & 29 & 66 & 55 \\
\hline 14 & 183 & 168 & 30 & 65 & 51 \\
\hline 15 & 179 & 172 & & & \\
\hline 16 & 166 & 159 & Mean: & 183.7 & 151.6 \\
\hline
\end{tabular}

- Combination of reports from 49 observers: see page 8.

- Dependent on observations at Zurich Observatory and 1 ts stations at Locarno and Arosa. 
Coronal observations at Cl1max, Colorado (5303A), east limb

\begin{tabular}{|c|c|c|c|c|c|c|c|c|c|c|c|c|c|c|c|c|c|c|c|c|c|c|c|c|c|c|c|c|c|c|c|c|c|c|c|c|c|c|}
\hline $\begin{array}{r}\text { Date } \\
\mathrm{GCT} \\
\end{array}$ & \multicolumn{18}{|c|}{ Degrees north of the solar oquator } & & \multicolumn{19}{|c|}{ Degrees south of the solar equator } \\
\hline & & & & & & & & & & & & & & & & & & & & & & & & & & & & & & & & & & & & & & \\
\hline 1.6 & $\mathrm{x}$ & & & $\mathrm{x}$ & $x$ & 1 & 2 & 3 & 3 & 4 & 6 & & 10 & 14 & 22 & 20 & 18 & & 7 & 8 & 8 & & 910 & & 9 & 8 & 7 & $\mathrm{x}$ & $\mathrm{x}$ & $\mathrm{x}$ & $x$ & $\bar{x}$ & $\mathbf{I}$ & $\mathrm{x}$ & $\mathrm{x}$ & $\mathrm{x}$ & $\mathrm{x}$ & $\mathrm{x}$ \\
\hline 4.71 & $\mathrm{x}$ & $\mathrm{x}$ & $\mathrm{x}$ & $\mathrm{x}$ & $\mathrm{x}$ & $\mathrm{x}$ & - & - & - & - & - & 1 & 4 & 5 & 7 & 9 & 10 & 12 & II & 10 & 9 & & & 8 & 7 & 6 & 5 & 4 & 1 & - & - & - & - & - & - & - & - & - \\
\hline 5.7 & - & - & - & - & - & - & - & - & - & 1 & 3 & 7 & 8 & 10 & & 12 & 13 & 13 & 12 & 11 & 10 & & 85 & 5 & 4 & 3 & 3 & 2 & 2 & 1 & - & - & - & - & - & - & - & - \\
\hline 6.7 & - & - & - & - & - & - & - & - & 1 & 3 & 7 & 9 & 11 & 15 & 17 & 19 & 18 & 14 & 9 & 11 & 1? & & 4 & 3 & 2 & 2 & 3 & 3 & 3 & 2 & 1 & - & - & - & - & - & - & - \\
\hline 7.6 & - & - & - & - & - & - & - & - & 1 & 3 & 3 & 4 & & 14 & 20 & 24 & 22 & 14 & 10 & 11 & 12 & & & 2 & 2 & 3 & 3 & 2 & 1 & - & 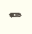 & 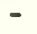 & - & - & - & - & - & - \\
\hline 8.6 & - & - & - & - & - & - & - & - & - & - & 1 & 3 & 4 & 9 & 15 & 18 & 20 & 16 & 9 & 8 & 8 & & & 6 & 5 & 5 & 4 & 3 & 1 & - & - & - & - & - & - & - & - & - \\
\hline 9.6 & - & - & - & - & - & - & - & 1 & 2 & 3 & 3 & 3 & 5 & 10 & 18 & 17 & 19 & 15 & 13 & 12 & 13 & 16 & 614 & 4 & 3 & 2 & 3 & 3 & 1 & - & 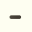 & - & - & - & - & - & - & - \\
\hline 10.8 & - & - & - & - & - & I & 3 & 3 & 4 & 4 & 8 & & 10 & 15 & $2 ?$ & 23 & 18 & 14 & 12 & 18 & 15 & 17 & 715 & 51 & 0 & 4 & 3 & 3 & 3 & $=$ & & - & - & - & - & - & - & - \\
\hline 11.7 & $\mathrm{x}$ & $\mathrm{x}$ & $\mathrm{X}$ & $\mathrm{X}$ & - & - & - & 1 & 2 & 3 & 3 & 3 & 9 & 13 & 18 & 30 & 20 & 14 & 13 & 12 & 12 & 13 & 313 & 31 & 21 & 10 & 9 & $\mathrm{x}$ & $x$ & $\bar{\pi}$ & $\mathrm{x}$ & $\mathrm{x}$ & $\mathrm{x}$ & $\mathrm{x}$ & $\bar{x}$ & $x$ & $\bar{x}$ & $x$ \\
\hline 12.9 & - & - & - & - & - & - & - & - & - & - & 1 & 3 & 6 & 15 & 20 & 23 & 20 & 13 & 12 & 10 & 9 & 9 & 910 & 01 & 10 & 7 & 3 & 1 & - & - & & 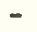 & - & - & - & - & - & - \\
\hline 14.7 & - & - & - & 1 & 2 & 2 & 2 & 2 & 2 & 2 & 3 & 10 & 17 & 28 & 30 & 35 & 30 & 30 & 17 & 15 & 33 & 34 & 425 & 51 & 31 & 11 & $1 ?$ & 12 & 9 & 8 & 6 & 5 & 2 & 1 & - & - & - & - \\
\hline 15.7 & - & - & - & - & 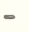 & - & - & - & - & 1 & 2 & 1 & 9 & 11 & 12 & 14 & 16 & 17 & 13 & 13 & 13 & 30 & 025 & 51 & 2 & 9 & 8 & 7 & 5 & 4 & 3 & 3 & 2 & 1 & - & - & - & - \\
\hline 16.7 & - & - & - & - & - & - & - & - & - & - & - & 1 & 6 & 16 & 16 & 15 & 15 & 15 & 14 & 16 & 16 & 32 & 230 & 01 & 181 & 14 & 12. & 6 & 3 & 1 & - & - & - & - & - & - & - & - \\
\hline 17.7 & - & - & - & - & - & - & - & $=$ & - & - & - & 1 & 5 & 10 & 14 & 13 & 13 & 12 & 11 & 1? & 14 & 14 & 413 & 31 & 121 & $1 ?$ & 10 & 9 & 8 & & - & - & - & - & - & - & - & - \\
\hline 18.7 & $\mathrm{x}$ & $\mathrm{x}$ & $\mathrm{x}$ & $\mathrm{x}$ & $\mathrm{x}$ & $\mathrm{x}$ & $\mathrm{x}$ & $\pi$ & X & 8 & $x$ & $x$ & $x$ & 18 & 20 & 24 & 23 & 17 & 12 & 15 & 25 & 24 & 424 & 42 & 201 & 11 & 8 & 1 & - & - & 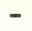 & - & - & - & - & - & - & - \\
\hline 19.6 & - & - & - & - & - & - & - & - & - & - & - & 1 & 3 & 10 & 18 & 22 & 24 & 19 & 19 & 21 & 25 & 24 & 420 & 01 & 191 & 15 & 5 & 2 & 1 & - & - & - & - & - & - & - & - & - \\
\hline 20.7 & $\mathrm{x}$ & $\mathrm{x}$ & $\mathrm{x}$ & $\bar{x}$ & $\mathrm{X}$ & $\mathrm{x}$ & $\mathrm{x}$ & $\mathrm{x}$ & $\mathrm{x}$ & $x$ & $\mathrm{x}$ & $\mathrm{x}$ & $\mathrm{X}$ & $\mathrm{x}$ & $\bar{x}$ & $x$ & $x$ & $\mathrm{x}$ & $\mathrm{x}$ & $\approx$ & $\mathrm{x}$ & & $\begin{array}{cc}x & x\end{array}$ & $\mathrm{x}$ & $\bar{x}$ & $\mathrm{x}$ & $\bar{x}$ & x & x & $x$ & & & $\mathrm{x}$ & X & $x$ & $x$ & $\mathrm{x}$ & $x$ \\
\hline $21 . \hat{k}$ & $\mathrm{x}$ & $\mathrm{x}$ & $\mathrm{x}$ & $x$ & $x$ & - & - & - & - & - & - & - & .. & 1 & 5 & 7 & 9 & 10 & 8 & 8 & 8 & & 87 & 7 & 7 & 6 & 5 & 1 & - & - & - & - & - & - & - & - & - & - \\
\hline 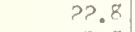 & - & - & - & - & - & - & 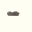 & 1 & 2 & 2 & 3 & 3 & 5 & d. & & 12 & 17 & 17 & 16 & 13 & in & 10 & ก 11 & 11 & 2 & 8 & 7 & 8 & 8 & 3 & & - & - & - & - & - & - & - \\
\hline 23.8 & - & - & - & - & - & - & - & - & 1 & 2 & 2 & 2 & 3 & 4 & & 10 & 13 & 12 & 12 & 13 & 10 & & 55 & 5 & 7 & 8 & 8 & 6 & 7 & 1 & - & - & - & - & - & - & - & - \\
\hline 24.8 & $\pi$ & $\mathrm{x}$ & - & - & - & - & - & - & - & - & - & - & - & 1 & & 8 & 8 & 9 & 11 & 13 & 1? & 12 & & 3 & 3 & 1 & - & - & $=$ & - & - & - & - & - & - & - & - & - \\
\hline 25.7 & - & - & - & - & - & - & _ & - & - & - & - & 1 & 2 & 4 & & 14 & 15 & 15 & 13 & 12 & 13 & 15 & 514 & 4 & & 1 & - & & & & & - & 1 & - & & & & \\
\hline 26.7 & $x$ & $\mathrm{x}$ & $\mathrm{x}$ & $\mathrm{x}$ & $\mathrm{X}$ & $\mathrm{X}$ & $\mathrm{x}$ & $\mathrm{x}$ & $\mathrm{x}$ & $\mathrm{x}$ & $\mathrm{x}$ & $\mathrm{x}$ & $\mathrm{X}$ & $x$ & $\mathrm{x}$ & $x$ & $\mathrm{x}$ & $\mathrm{x}$ & $\mathrm{x}$ & $\mathrm{x}$ & $x$ & & & $\mathrm{x}$ & $\bar{x}$ & $x$ & $x$ & x & X & $x$ & x & $x$ & $x$ & $x$ & $x$ & $x$ & $\mathrm{x}$ & X \\
\hline 27.7 & - & - & - & - & - & 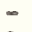 & 1 & 2 & 3 & 5 & 8 & 10 & 12 & 14 & 18 & 32 & 25 & 28 & 23 & 18 & 16 & 19 & 922 & 21 & 14 & 12 & 4 & 3 & 4 & 5 & 5 & & 3 & ๘ & 1 & 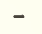 & - & - \\
\hline 0 & - & - & - & $=$ & - & 1 & 2 & 3 & 3 & 4 & 6 & & 12 & 15 & 18 & 21 & 19 & 19 & 19 & 15 & 17 & 27 & 720 & 01 & & 12 & 8 & 4 & 2 & & & 3 & 1 & - & - & $=$ & $=$ & \\
\hline 29.8 & $x$ & $\mathrm{x}$ & $x$ & $\mathrm{x}$ & $x$ & - & - & 1 & 2 & 5 & 8 & 12 & 17 & 19 & 20 & 21 & 17 & 18 & 18 & 18 & & & & & 10 & 8 & 3 & $?$ & 1 & & & & & - & - & $x$ & $\bar{x}$ & $\pi$ \\
\hline 30.7 & $x$ & - & - & 1 & 3 & 3 & & 2 & 1 & 2 & & & 15 & 18 & 19 & 20 & 20 & 12 & 19 & 18 & 24 & & 720 & 01 & & 12 & 8 & & & ? & c. & & & 5 & $x$ & $x$ & $x$ & $x$ \\
\hline
\end{tabular}

\section{Table 73a}

Coronal observations at Climax, Colorado (6374A), east limb

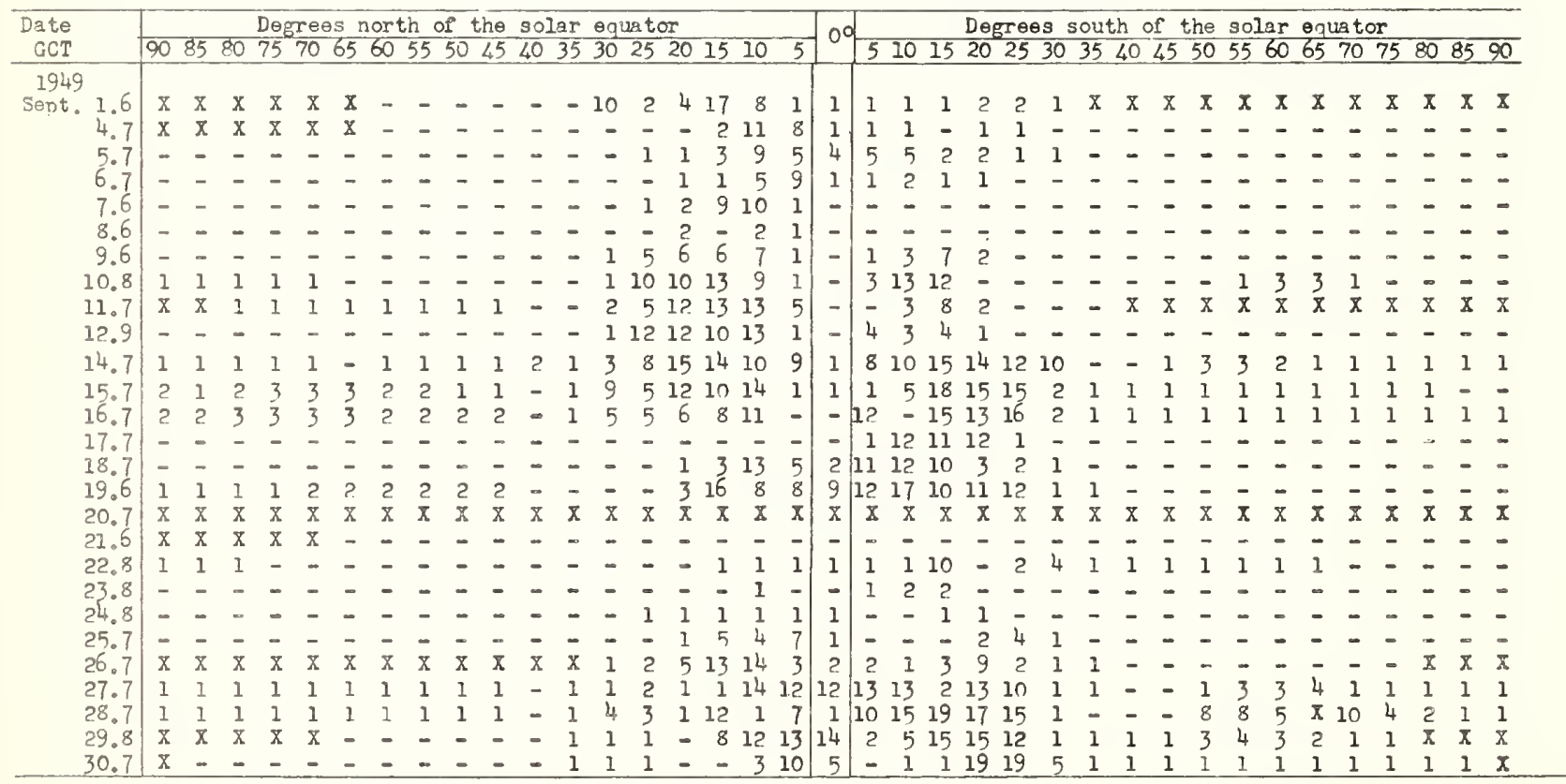




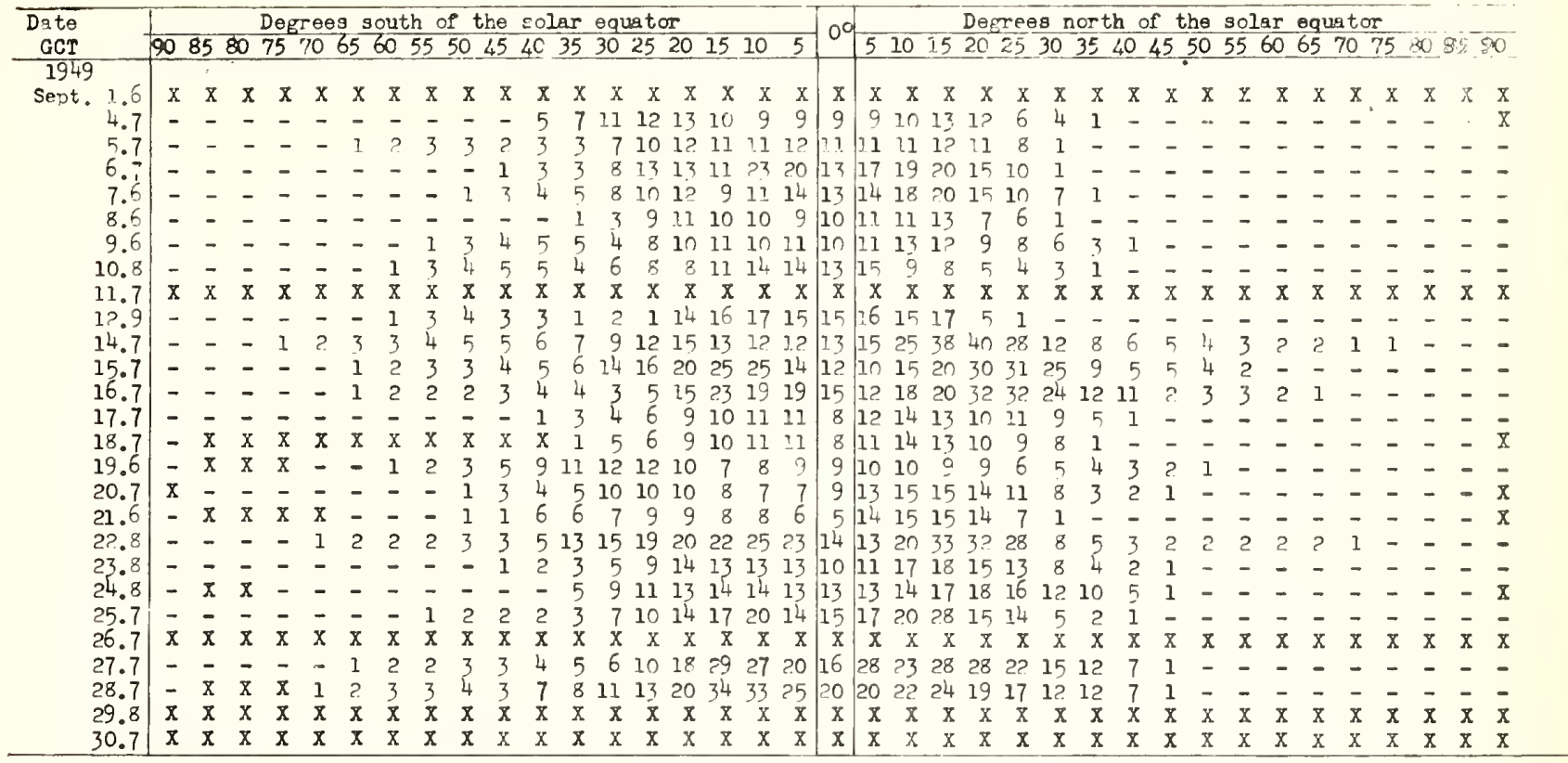

Table 73b

Coronal observations at Climax, Colorado (6374A), west limb

\begin{tabular}{|c|c|c|c|c|c|c|c|c|c|c|c|c|c|c|c|c|c|c|c|c|c|c|c|c|c|c|c|c|c|c|c|c|c|c|c|c|c|c|c|}
\hline $\begin{array}{r}\text { Dete } \\
\text { GCT }\end{array}$ & \multicolumn{19}{|c|}{ Degrees south of the solar equator } & 09 & \multicolumn{18}{|c|}{ Degrees north of the solar equator } & \\
\hline 1949 & & & & & & & & & & & & & & & & & & & & & & & & & & & & & & & & & & & & & & & \\
\hline 1.6 & $\mathrm{x}$ & & $\mathbf{z}$ & $\mathrm{x}$ & $\mathrm{x}$ & $x$ & $\mathrm{x}$ & $\mathrm{x}$ & $\mathrm{x}$ & $\mathrm{x}$ & $\mathrm{x}$ & $\mathrm{x}$ & $\mathrm{x}$ & $\mathrm{x}$ & $\mathrm{x}$ & & & $\mathrm{x}$ & $\mathrm{x}$ & $\mathrm{x}$ & $\mathrm{x}$ & & $\mathrm{x}$ & $\mathrm{x}$ & $\mathrm{x}$ & $\mathrm{x}$ & $\mathrm{x}$ & $\mathrm{x}$ & $\mathrm{x}$ & $\mathrm{x}$ & $\mathrm{x}$ & $\mathrm{x}$ & $\mathrm{x}$ & & $\mathrm{x}$ & $\mathrm{x}$ & $\mathrm{x}$ & $\mathrm{x}$ & \\
\hline & - & - & - & - & - & 1 & 1 & 1 & 1 & 2 & 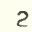 & 7 & 1 & 1 & 1 & & 2 & 1 & 1 & 2 & 1 & 2 & 1 & 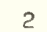 & 1 & $\bar{x}$ & $\mathrm{x}$ & $\mathrm{x}$ & $\mathrm{x}$ & $\mathrm{x}$ & $\mathrm{x}$ & $\mathrm{x}$ & 7 & & $\mathrm{x}$ & x & $\bar{x}$ & $x$ & \\
\hline 5.7 & - & - & - & - & - & 1 & 1 & 1 & 1 & 1 & 1 & - & - & 1 & - & & - & & 10 & 1 & 1 & 9 & 1 & 1 & - & - & - & - & - & - & - & - & - & & - & - & - & - & \\
\hline 6 & - & - & - & - & 1 & I & 1 & 2 & I & 1 & 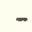 & - & - & 2 & 1 & & & 11 & 13 & 3 & I & 9 & 8 & 5 & 1 & - & - & - & - & - & - & - & - & & - & - & - & - & - \\
\hline 7. & - & - & - & - & - & 1 & 1 & 2 & 2 & 1 & - & 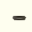 & 1 & 3 & 8 & & & & 14 & 2 & 3 & - & 3 & - & - & - & - & - & - & - & - & - & - & & - & - & & - & - \\
\hline & - & - & - & - & - & - & - & - & - & - & - & - & 1 & $i$ & 3 & & 4 & 4 & 3 & 4 & 4 & - & 7 & 1 & - & - & - & - & - & - & - & - & - & & - & - & - & - & - \\
\hline 9 & - & - & - & 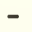 & - & - & - & - & - & - & 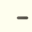 & - & - & - & 1 & & & 6 & - & & - & - & I & - &. & - & - & - & - & - & - & - & - & & - & - & - & - & - \\
\hline 10.8 & - & - & - & - & - & - & - & - & - & - & - & - & - & - & 2 & & & 10 & 3 & & 2 & 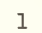 & 1 & - & - & - & - & - & - & - & - & - & - & & - & - & - & - & 1 \\
\hline .7 & $x$ & $x$ & $x$ & $x$ & $x$ & $x$ & 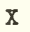 & $x$ & X & X & $\mathrm{x}$ & X & $x$ & $\mathrm{x}$ & X & & z & $x$ & $x$ & & $\bar{\chi}$ & $\bar{x}$ & $\bar{x}$ & $x$ & $x$ & $\pi$ & $x$ & $x$ & $x$ & $x$ & $x$ & $x$ & $\mathrm{x}$ & & $x$ & $\mathrm{x}$ & $\mathrm{x}$ & $x$ & $\bar{x}$ \\
\hline 12.9 & - & - & - & - & - & - & I & 1 & 1 & - & - & - & 1 & 2 & - & 1 & & 3 & 1 & & 7 & 8 & 5 & 1 & ? & - & - & - & & - & - & - & - & & - & - & - & - & \\
\hline 14.7 & 1 & I & 1 & - & 1 & 2 & ? & 3 & 3 & I & 1 & 1 & 1 & 9 & 8 & & 7 & 8 & 1 & 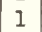 & 13 & & 14 & 12 & 8 & 2 & - & - & - & - & 1 & 1 & 1 & & 7 & 1 & 1 & 1 & 1 \\
\hline 5.7 & - & - & - & - & 2 & 2 & 3 & 4 & 9 & 5 & 1 & - & ] & 8 & 12 & 15 & & 16 & 8 & 4 & 3 & & 22 & 13 & 10 & 5 & ) & - & - & - & - & - & 3 & & 3 & 2 & 2 & 2 & \\
\hline 16.7 & 1 & 1 & 1 & 1 & 1 & 1 & 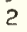 & 3 & 3 & 3 & I & g. & - & - & 10 & 19 & & 15 & 3 & 1 & 6 & 3 & 1 & 1 & 12 & 8 & 8 & 1 & - & - & - & 1 & 2 & & 3 & 3 & 3 & 3 & 2 \\
\hline 17.7 & - & - & - & - & - & - & - & - & 1 & 1 & 2 & I & 3 & 5 & 8 & & 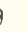 & 1 & 1 & - & in & 3 & 1 & 1 & 7 & 1 & - & - & - & - & - & - & - & & - & - & - & - & - \\
\hline 18. & - & - & - & - & - & - & 1 & 1 & 1 & 1 & 2 & 2 & 3 & 4 & 5 & 5 & & 6 & & 1 & 8 & 10 & 3 & ]. & $E$ & - & - & - & - & - & & - & - & & - & & & - & \\
\hline 19.6 & - & $x$ & $x$ & 1 & 1 & 1 & 1 & 1 & 2 & 2 & ? & 1 & 1 & 3 & 6 & & & 2 & ? & 1 & 1 & & 10 & 2 & - & - & - & ـ. & - & - & - & - & - & & - & & & - & 1 \\
\hline 20.7 & $\pi$ & - & - & - & - & - & - & - & - & - & - & - & - & - & - & - & - & - & - & & 1 & 9 & 4 & ? & 1 & - & - & - & - & - & - & - & - & & - & & - & - & \\
\hline 21.6 & - & $\bar{x}$ & 7 & $\pi$ & X & - & - & - & - & - & - & 1 & & 1 & 1 & & & , & & & 1 & 4 & 3 & & 7 & - & - & - & - & - & & - & - & & - & & & - & \\
\hline 22.8 & - & - & - & - & 1 & 1 & 1 & 1 & 2 & 2 & i & - & - & - & 5 & & 1 & & 14 & 5 & & 10 & 2 & 5 & 1 & - & - & - & - & - & - & - & - & & - & & - & 1 & 1 \\
\hline 23 & - & - & - & - & - & - & - & - & - & - & - & 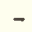 & - & - & - & & & 3 & 4 & & 1 & 3 & 2 & 1 & - & - & - & - & - & - & - & - & - & & & & & - & $=$ \\
\hline 24.8 & - & - & 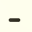 & - & 3 & 1 & 2 & 2 & 2 & & 3 & 2 & 1 & 1 & 4 & & & 7 & & & & & 2 & $=$ & . & - & - & . & - & & & - & & & & & & & \\
\hline 5 & - & - & - & - & - & - & 1 & 1 & 1 & 2 & 2 & 2 & 2 & - & 1 & & 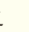 & 1 & 2 & 4 & 4 & 4 & 1 & 1 & 1 & 2 & $\bar{F}$ & 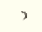 & 1 & - & - & - & - & & - & & & & - \\
\hline 26.7 & X & $x$ & $x$ & $x$ & $x$ & $x$ & 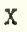 & $\bar{x}$ & $\bar{x}$ & X & $x$ & $x$ & $\mathrm{x}$ & $\mathrm{x}$ & $x$ & $y$ & & $\bar{X}$ & $\mathrm{x}$ & & & $x$ & $\bar{x}$ & X & $\mathrm{v}$ & $\bar{x}$ & $\mathrm{X}$ & 6 & $\pi$ & X & & $\mathrm{x}$ & $x$ & & $\mathrm{x}$ & X & X & $x$ & $x$ \\
\hline 27.7 & 1 & 1 & 1 & 1 & 1 & 1 & 1 & 1 & 1 & 2 & $I$ & 1 & 3 & 6 & 8 & 10 & & 11 & 13 & 8 & - & 1 & 3 & 4 & 4 & 1 & - & . & - & .- & - & 1 & I & & I & 1 & 1 & 1 & \\
\hline 28. & ? & 2 & - & 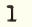 & 3 & 8 & 8 & 10 & 8 & 8 & 6 & & 13 & 14 & 14 & 19 & & & 12 & ? & & 14 & 12 & 8 & 8 & 4 & 1 & .. & & & & - & 1 & & & & & & \\
\hline 29.8 & $\bar{\lambda}$ & $\bar{x}$ & $\pi$ & $\bar{x}$ & $\bar{x}$ & $x$ & 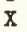 & $x$ & $\mathrm{x}$ & $\pi$ & $x$ & $x$ & $\pi$ & $x$ & $x$ & +1 & & $\mathrm{x}$ & $\mathrm{X}$ & $x$ & X & $x$ & $\mathrm{X}$ & $\mathrm{X}$ & $\mathrm{X}$ & $\bar{x}$ & $\mathrm{X}$ & $x$ & $x$ & $\mathrm{x}$ & . & $x$ & X & & $\mathrm{x}$ & x & $\pi$ & $\bar{X}$ & \\
\hline 0. & $x$ & $x$ & $x$ & $x$ & $x$ & $x$ & $x$ & $\bar{x}$ & $\bar{x}$ & X & 2. & $\boldsymbol{x}$ & $x$ & $X$ & X & & & $\mathrm{x}$ & $\mathrm{x}$ & $x$ & & $x$ & X & $x$ & X & $x$ & $x$ & & & $\mathrm{X}$ & & $x$ & & & & & & & \\
\hline
\end{tabular}


Coronal observations at Climi: $x$, Colorado (6704A), east limb

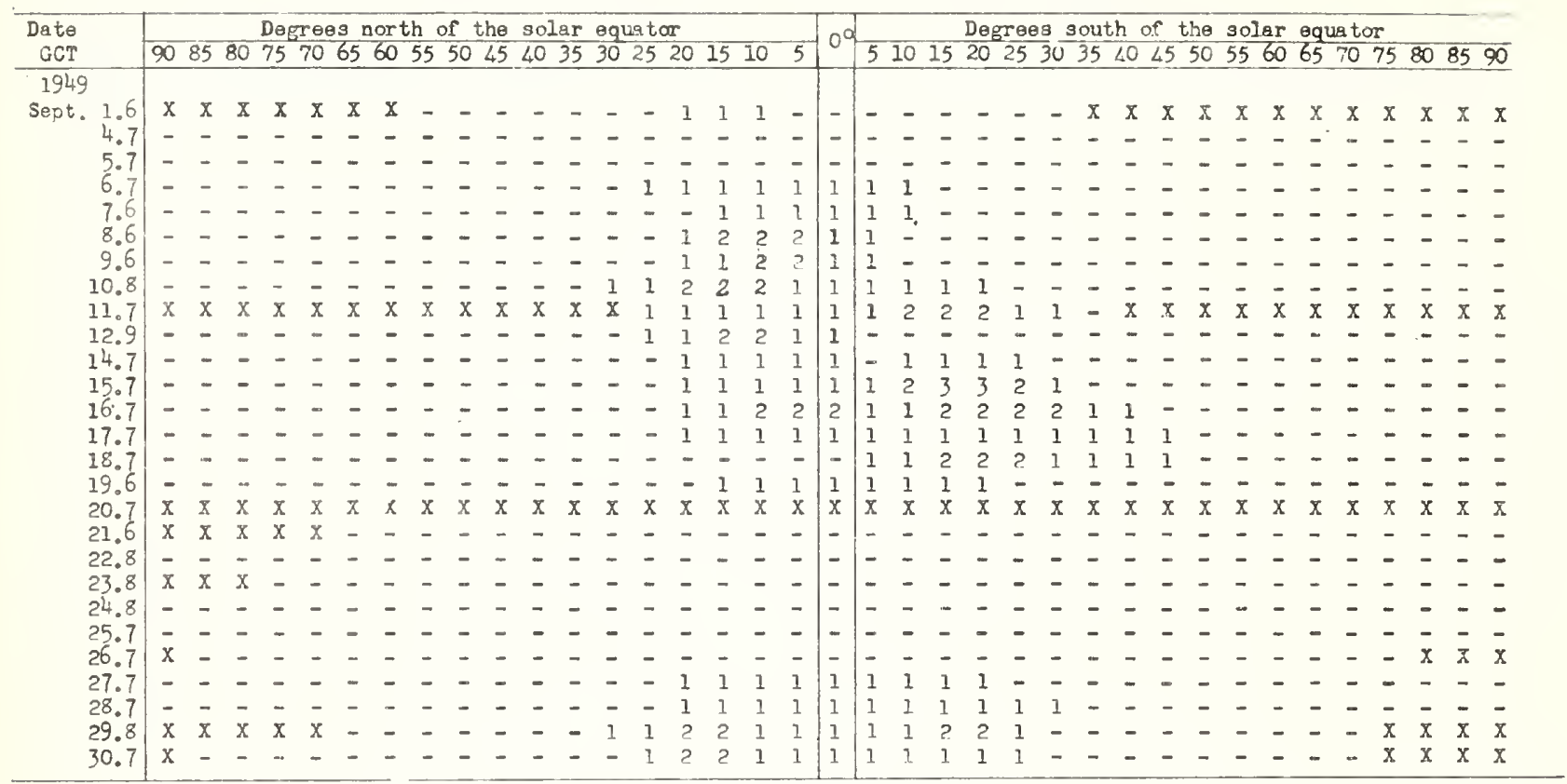


Coronal observations at Cl1max, Colorido (6704A), west 11mb

\begin{tabular}{|c|c|c|c|c|c|c|c|c|c|c|c|c|c|c|c|c|c|c|c|c|c|c|c|c|c|c|c|c|c|c|c|c|c|c|c|c|c|c|c|c|}
\hline \multirow{2}{*}{\multicolumn{2}{|c|}{$\begin{array}{r}\text { Date } \\
\text { GCT }\end{array}$}} & \multicolumn{18}{|c|}{ Degrees south of the solar enuator } & \multirow{2}{*}{09} & \multicolumn{19}{|c|}{ Degrees north of the solar equator } & \\
\hline & & & & & & & 65 & 60 & & 50 & 45 & 40 & 35 & 30 & 25 & & 15 & 10 & 5 & & & 10 & 15 & & & & & & & 50 & & & & & & & 80 & 85 & 90 & \\
\hline 1949 & & & & & & & & & & & & & & & & & & & & & & & & & & & & & & & & & & & & & & & & \\
\hline Sept. & 1.6 & $\mathrm{x}$ & $x$ & $\mathrm{x}$ & $\mathrm{x}$ & $x$ & $\mathrm{x}$ & $x$ & $\mathrm{x}$ & $\mathrm{x}$ & $x$ & $x$ & $\mathrm{x}$ & $\mathrm{x}$ & $\mathrm{X}$ & $\mathrm{x}$ & $\mathrm{X}$ & 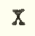 & $\mathrm{x}$ & $x$ & $x$ & $x$ & $\mathrm{X}$ & $x$ & $\mathrm{X}$ & $x$ & $x$ & $x$ & $x$ & $x$ & $x$ & $x$ & $x$ & X & & $\pi$ & $x$ & $x$ & & \\
\hline & 4.7 & - & - & - & - & - & - & - & - & - & - & - & - & - & - & - & - & - & - & - & - & - & - & - & - & - & - & - & - & - & - & - & - & - & & - & - & - & 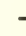 & \\
\hline & 5.7 & - & - & - & - & - & - & - & - & - & - & - & - & - & - & - & - & - & - & - & - & - & - & - & - & - & - & - & - & - & - & - & - & - & & - & - & - & - & \\
\hline & 6.7 & - & - & - & - & - & - & - & - & - & - & - & - & - & - & - & - & - & - & - & - & - & - & - & - & - & - & - & - & - & - & - & - & - & & - & - & - & - & \\
\hline & 7.6 & - & - & - & - & - & - & - & - & - & - & - & - & - & - & - & - & - & - & - & - & - & - & - & - & - & - & - & - & - & - & - & - & - & & - & - & - & - & \\
\hline & 8.6 & - & - & - & - & - & - & - & - & - & - & - & - & - & - & - & - & - & - & - & - & - & - & - & - & - & - & - & - & - & - & - & - & - & & - & - & - & - & \\
\hline & 9.6 & - & - & - & - & - & - & - & - & - & - & - & - & - & - & - & - & - & - & - & - & - & - & - & - & - & - & - & - & - & - & - & - & - & & - & - & - & - & \\
\hline & 10.8 & - & - & - & - & - & - & - & - & - & - & - & - & - & - & - & - & - & - & - & - & - & - & - & - & - & - & - & - & - & - & - & - & - & & - & - & - & - & \\
\hline & 11.7 & $x$ & $x$ & $x$ & $x$ & $x$ & $X$ & $x$ & $X$ & $x$ & $x$ & $\mathrm{X}$ & $x$ & $x$ & $x$ & $x$ & $x$ & $X$ & $\mathrm{X}$ & $\mathrm{X}$ & $\mathrm{X}$ & $\mathrm{X}$ & $\mathrm{X}$ & $\mathrm{X}$ & $\mathrm{X}$ & $x$ & $\mathrm{X}$ & $X$ & $\mathrm{X}$ & $x$ & $x$ & $\mathrm{X}$ & $x$ & $x$ & & X & $\mathrm{X}$ & $x$ & $y$ & \\
\hline & 12.9 & - & - & - & - & - & - & - & - & - & - & - & - & - & - & - & - & - & - & - & - & - & - & - & - & - & - & - & - & - & - & - & - & - & & - & - & - & - & \\
\hline & 14.7 & - & - & - & - & - & - & - & - & - & - & - & - & - & - & - & - & - & - & - & - & - & - & - & - & - & - & - & - & - & - & - & - & - & & - & - & - & - & \\
\hline & 15.7 & - & - & - & - & - & - & - & - & - & - & - & - & - & - & - & - & - & - & - & - & - & - & - & - & - & - & - & - & - & - & - & - & - & & - & - & - & 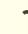 & \\
\hline & 16.7 & - & - & - & - & - & - & - & - & - & - & - & - & - & - & - & - & - & - & - & - & - & - & - & - & - & - & - & - & - & - & - & - & - & & - & - & - & - & \\
\hline & 17.7 & - & - & - & - & - & - & - & - & - & - & - & - & - & - & - & - & - & - & - & - & - & - & - & - & - & - & - & - & - & - & - & - & - & & - & - & - & 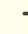 & \\
\hline & 18.7 & - & - & - & - & - & - & - & - & - & - & - & - & - & - & - & - & - & - & - & - & - & - & - & - & - & - & - & - & - & - & - & - & - & & - & - & - & 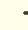 & \\
\hline & 19.6 & - & - & - & - & - & - & - & - & - & - & - & - & - & - & - & - & - & - & - & - & - & - & - & - & - & - & - & - & - & - & - & - & - & & - & - & - & 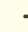 & \\
\hline & 20.7 & $x$ & $x$ & $\pi$ & $\pi$ & $x$ & $\mathrm{X}$ & $\mathrm{X}$ & $x$ & $x$ & $x$ & $x$ & $x$ & $x$ & $x$ & $x$ & $x$ & $x$ & $x$ & $x$ & $x$ & $x$ & $x$ & $x$ & $x$ & $x$ & $x$ & $x$ & $x$ & $x$ & $x$ & $x$ & $x$ & $x$ & & $x$ & $x$ & $x$ & & \\
\hline & 21.6 & - & - & - & - & - & - & - & - & - & - & - & - & - & - & - & - & - & - & - & - & - & - & - & - & - & - & - & - & - & - & - & - & - & & - & - & - & 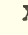 & \\
\hline & 22.8 & - & - & - & - & - & - & 1 & 1 & 1 & 1 & 1 & 1 & 1 & 2 & 2 & 2 & 3 & 3 & 3 & - & 2 & 2 & 2 & 2 & 1 & - & - & - & - & - & - & - & - & & - & - & - & & \\
\hline & 23.8 & - & - & - & - & - & - & - & - & - & - & - & - & - & 1 & 2 & 1 & 1 & 1 & 1 & 1 & 1 & 2 & 2 & 1 & 1 & - & - & - & - & - & - & - & - & & - & - & - & 8 & \\
\hline & 24.8 & - & - & - & - & - & - & - & - & - & - & - & - & - & - & - & - & - & - & - & - & 1 & 1 & 1 & 1 & 1 & $\overline{-}$ & - & - & - & - & - & - & - & & - & - & - & 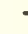 & \\
\hline & 25.7 & - & - & $=$ & - & - & - & $=$ & - & - & - & - & - & 1 & 1 & 1 & 1 & 1 & 1 & 1 & 2 & 2 & 2 & 2 & 1 & 1 & 1 & - & $=$ & $\overline{-}$ & $\overline{-}$ & - & $\overline{-}$ & - & & - & - & $\overline{-}$ & & \\
\hline & 26.7 & $x$ & $x$ & $X$ & $x$ & $x$ & $x$ & $x$ & $x$ & $x$ & $x$ & $x$ & $x$ & $x$ & $x$ & $x$ & $x$ & $x$ & $x$ & $X$ & $x$ & $X$ & $x$ & $x$ & $x$ & $x$ & $\bar{x}$ & $x$ & $x$ & $x$ & $\bar{x}$ & $\pi$ & $x$ & $\gamma$ & & $X$ & $x$ & $x$ & & \\
\hline & 27.7 & - & - & - & - & - & - & - & - & - & - & - & - & - & - & - & - & - & 1 & 1 & 2 & 1 & 1 & 1 & 1 & 1 & 1 & 1 & 1 & - & - & - & - & - & & - & - & - & . & \\
\hline & 28.7 & - & - & - & - & - & - & - & - & - & - & - & - & - & 1 & 1 & 2 & 2 & 1 & 1 & 1 & 1 & 1 & 1 & - & - & - & - & - & - & - & $\overline{-}$ & $\overline{-}$ & 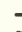 & & - & - & - & & \\
\hline & 29.8 & $x$ & $x$ & $X$ & $x$ & $x$ & $x$ & $X$ & $x$ & $x$ & $x$ & $x$ & $\mathrm{X}$ & $x$ & $x$ & $x$ & $x$ & $x$ & $x$ & $x$ & $\mathrm{X}$ & $x$ & $x$ & $\pi$ & $x$ & $x$ & $x$ & $X$ & $x$ & $\mathrm{x}$ & $x$ & $\%$ & $x$ & 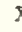 & & $x$ & $x$ & $x$ & & \\
\hline & 30.7 & $x$ & $x$ & $x$ & $\bar{x}$ & $x$ & $x$ & $x$ & $X$ & $x$ & $x$ & $x$ & $x$ & $x$ & $x$ & $x$ & $\mathrm{X}$ & $x$ & $x$ & $\mathrm{X}$ & $x$ & $X$ & $x$ & $x$ & $X$ & $x$ & $x$ & $x$ & $x$ & $x$ & $x$ & $x$ & $X$ & $y$ & & $\pi$ & $x$ & $\pi$ & & \\
\hline
\end{tabular}




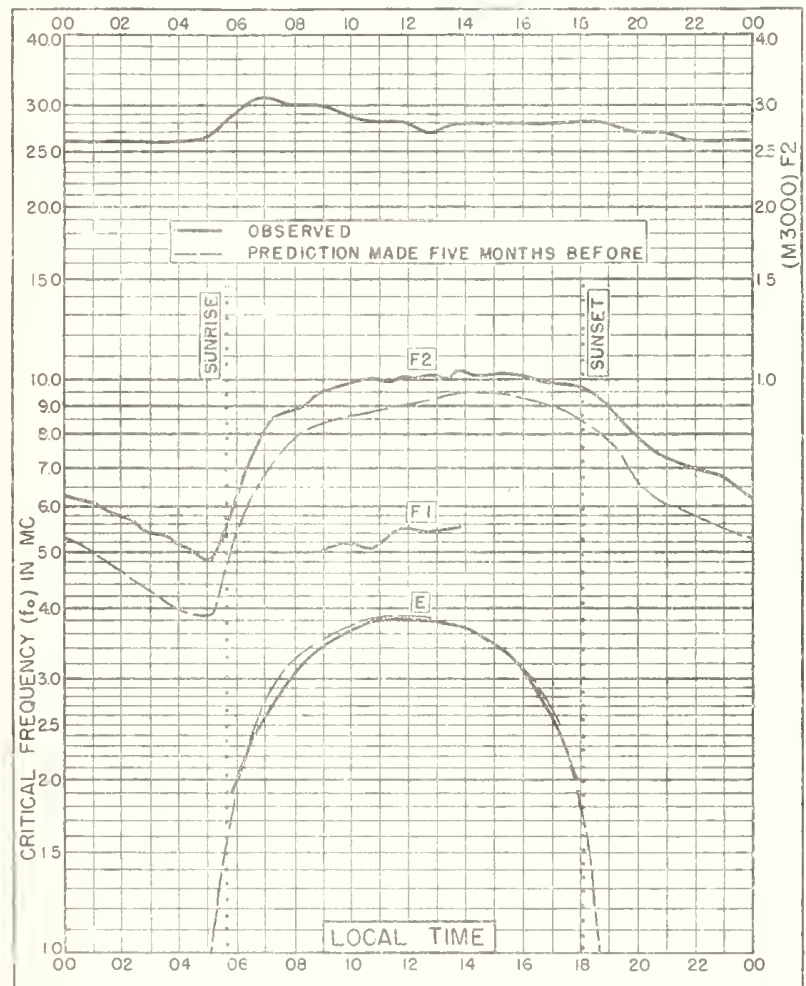

FIg. I. WASHING TOM, D. C

38. $7^{\circ} \mathrm{N}, 77.1^{\circ} \mathrm{W}$

SEPTEMBER 1949

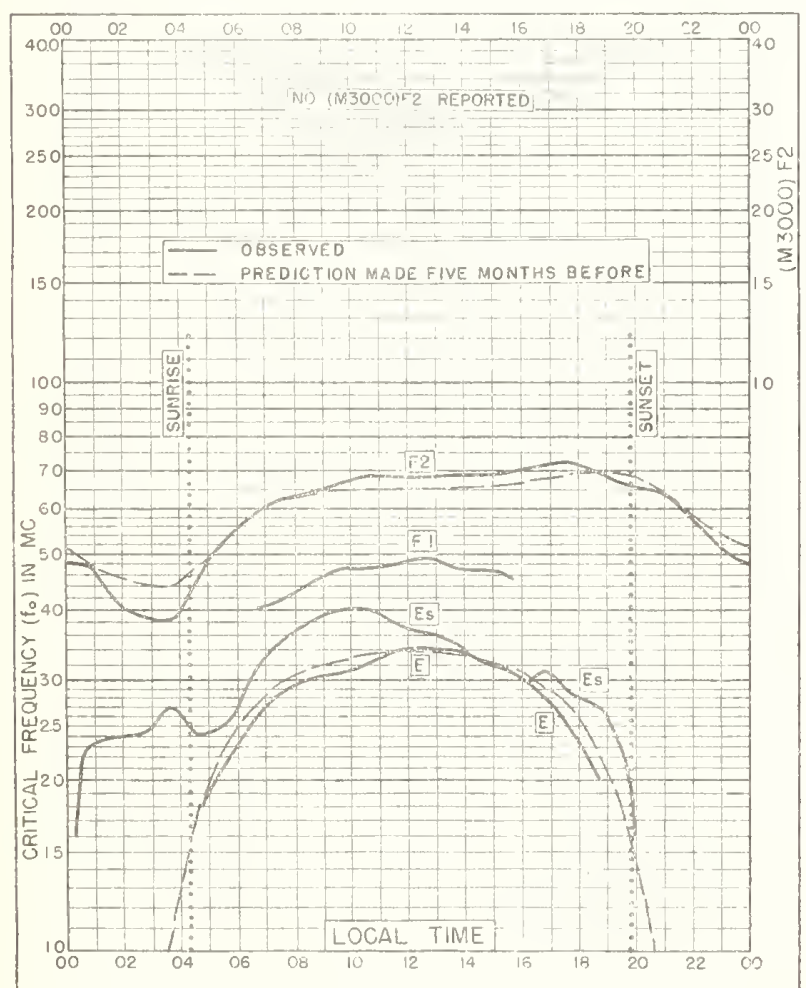

Fig. 3. OSLO, NORWAY

60. $0^{\circ} \mathrm{N}, 11.0^{\circ} \mathrm{E}$

AUGUST 1949
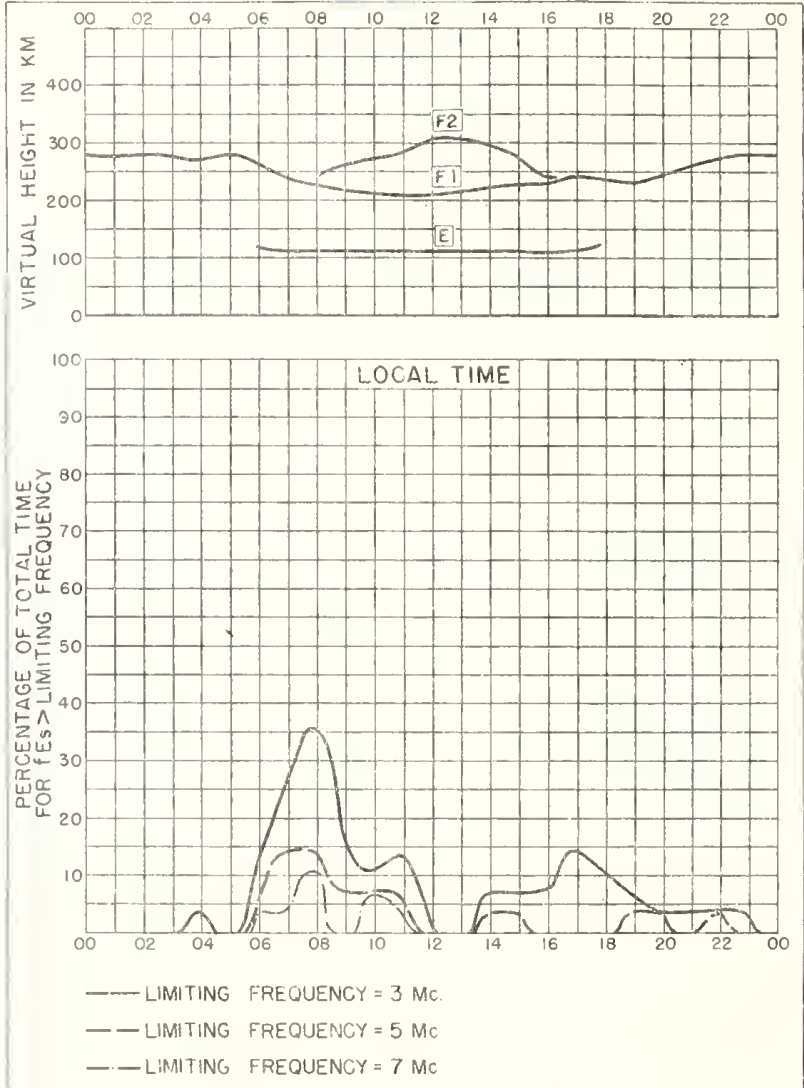

Fig. 2. WASHINGTON, D. C

SEPTEMBER 1949
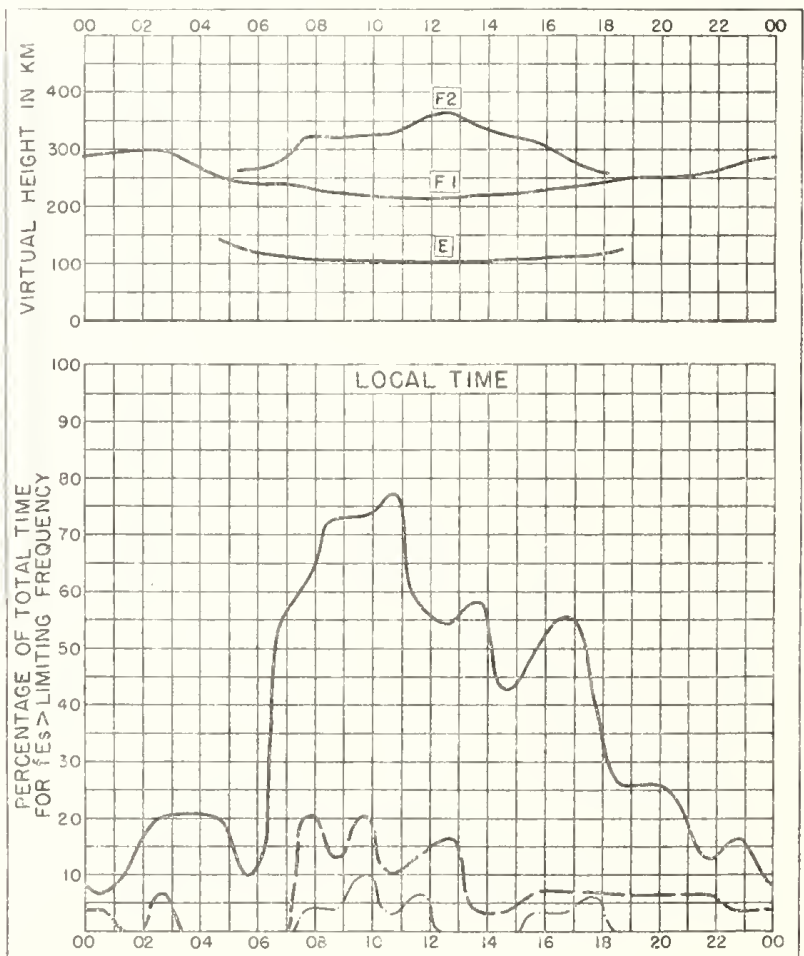

\section{- LIMITING FREQUENCY $=3 \mathrm{MC}$. \\ - LIMITING FREQUENCY $=5 \mathrm{MC}$. \\ - - LIMITING FREQUENCY $=7 \mathrm{MC}$}

Fig. 4. OSLO, NORWAY

AUGUST 1949 


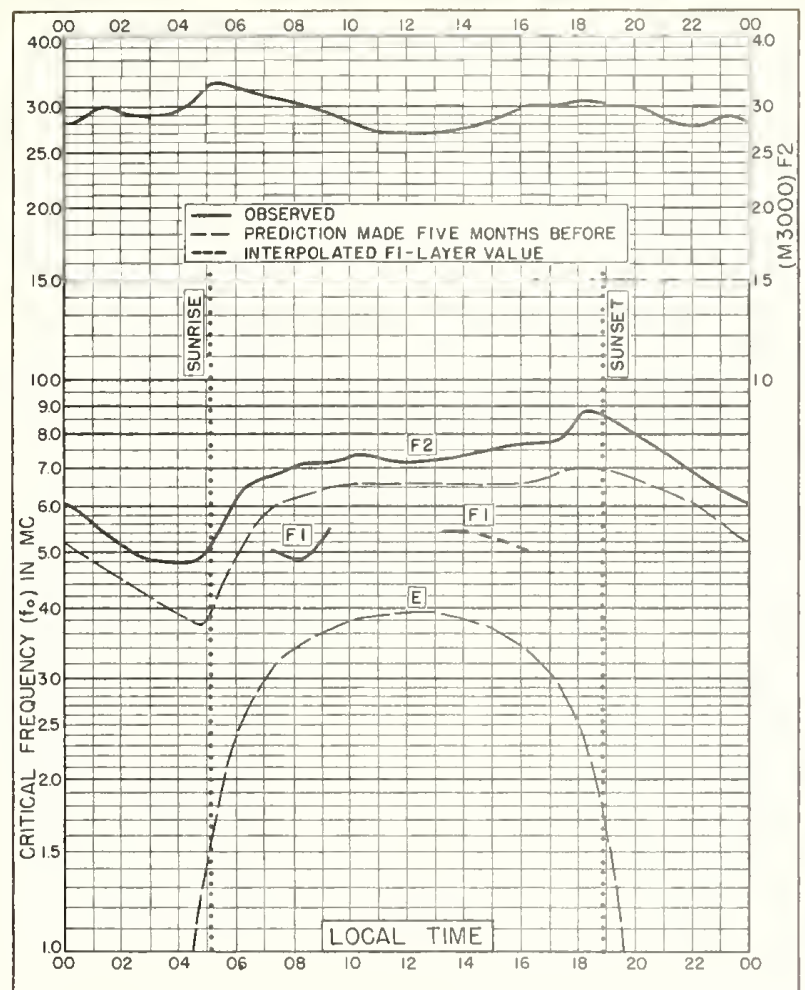

Fig. 5 BOSTON, MASSACHUSETTS

$42.4^{\circ} \mathrm{N}, 71.2^{\circ} \mathrm{W}$

AUGUST 1949

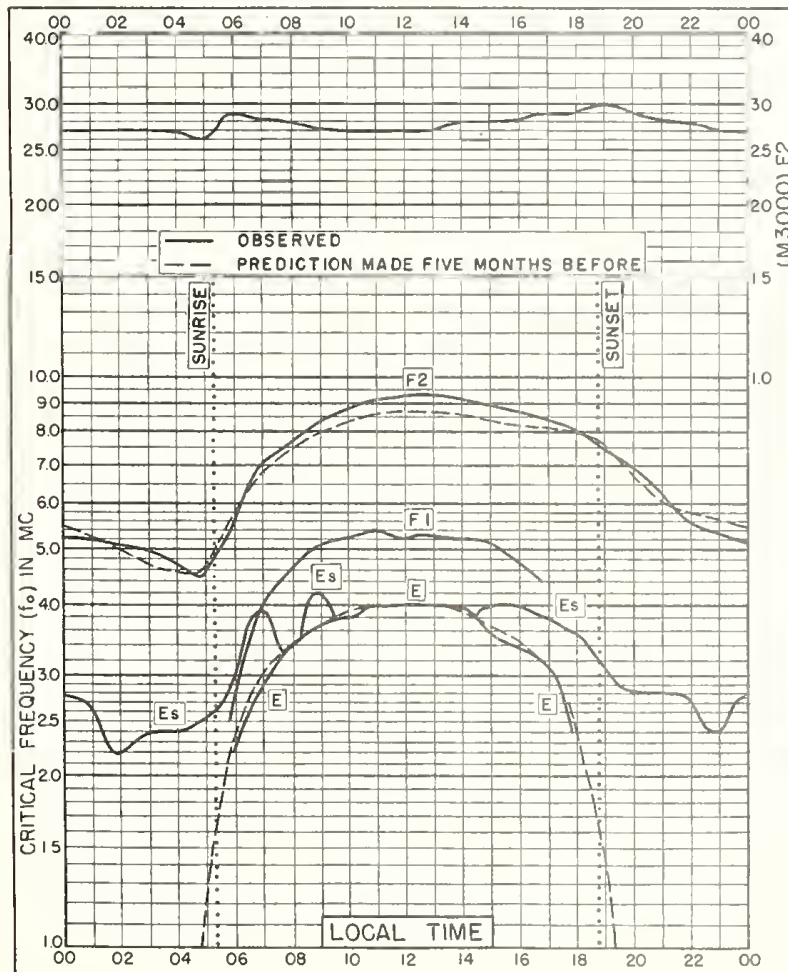

Fig. 7. SAN FRANCISCO, CALIFORNIA $37.4^{\circ} \mathrm{N}, 122.2^{\circ} \mathrm{W}$

AUGUST 1949
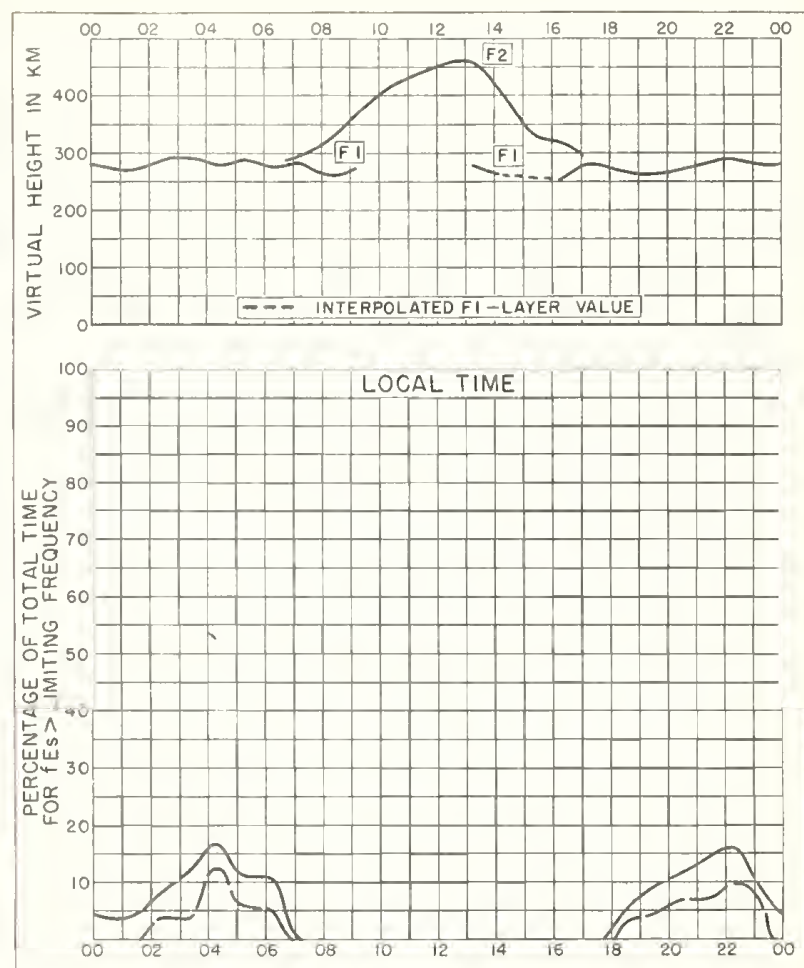

- LIMITING FREQUENCY $=3 \mathrm{MC}$.
- LIMITING FREQUENCY $=5 \mathrm{Mc}$.
- LIMITING FREQUENCY $=7 \mathrm{MC}$.

Fig.6. BOSTON, MASSACHUSETTS AUGUST 1949

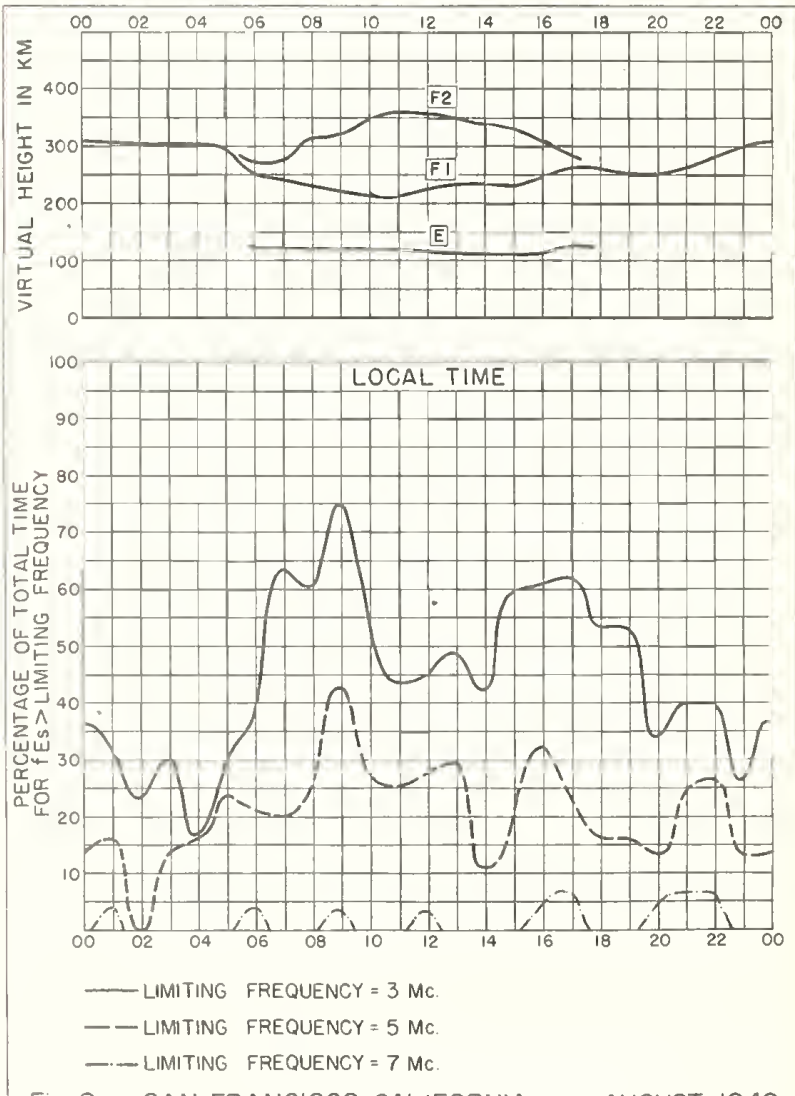

Fig 8. SAN FRANCISCO, CALIFORNIA AUGUST 1949 


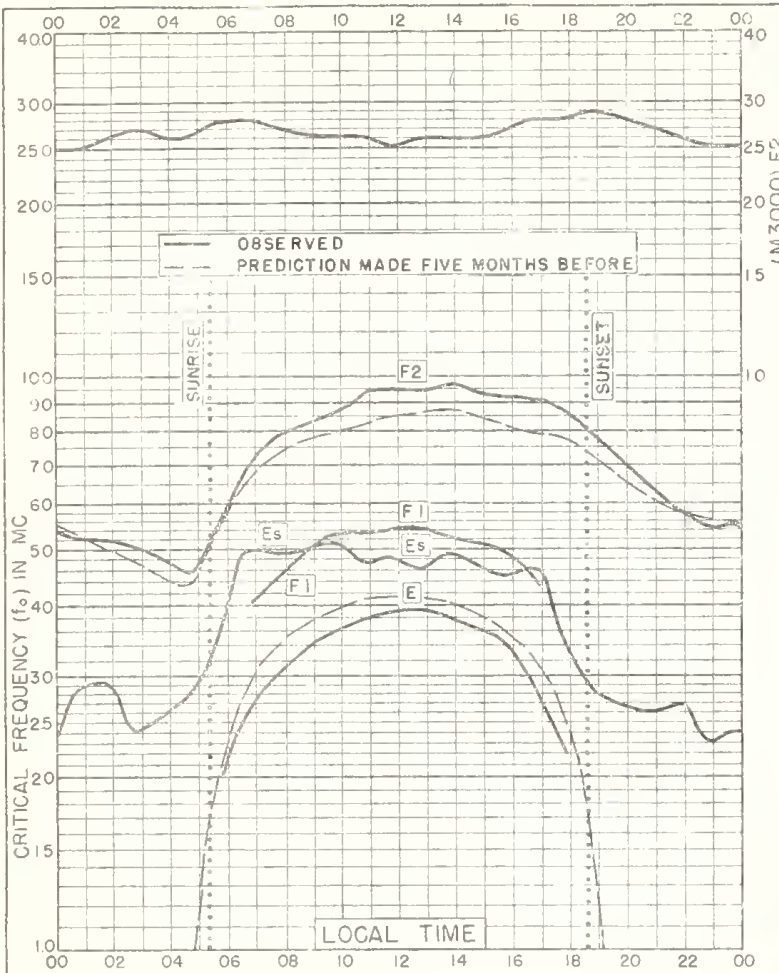

Fig. 9. WHITE SANDS, NEW MEXICO 32. $3^{\circ} \mathrm{N}, 106.5^{\circ} \mathrm{W}$

AUGUST 1949

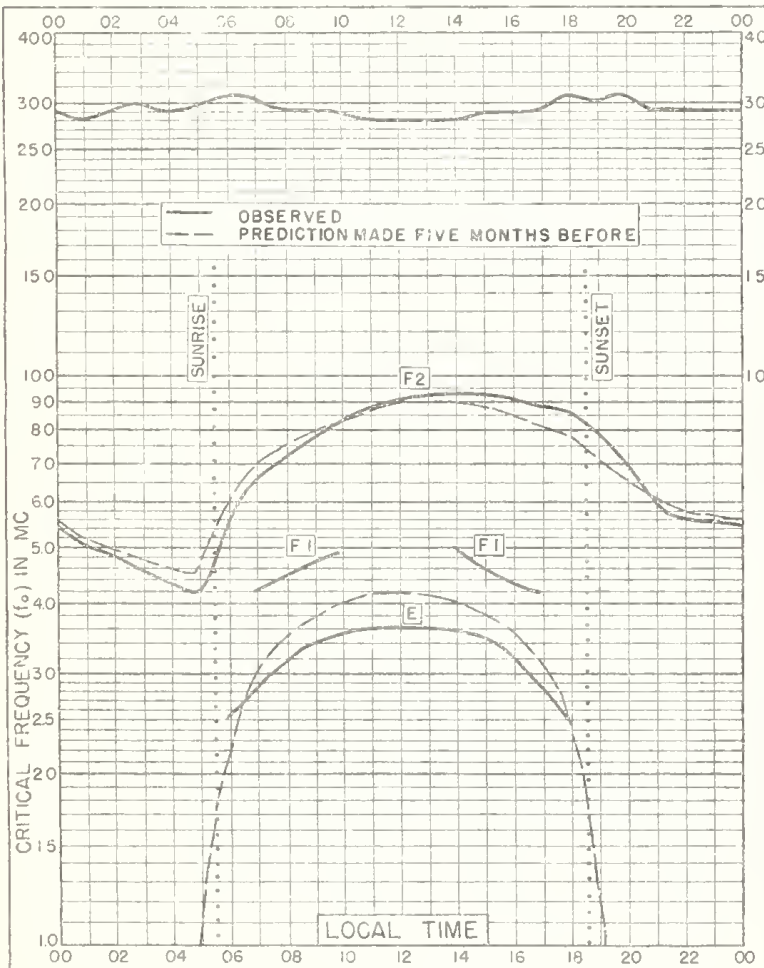

Fig. II. BATON ROUGE, LOUISIANA.

$30.5^{\circ} \mathrm{N}, 91.2^{\circ} \mathrm{W}$
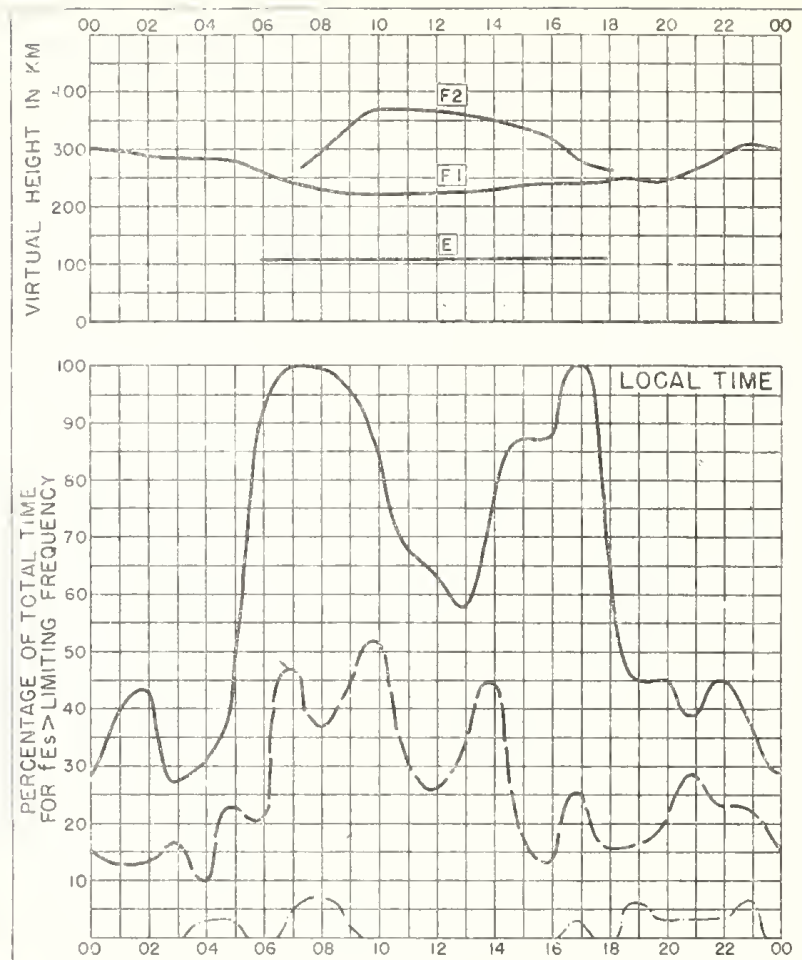

LIMITING FREQUENCY $=3$ MC.

- LIMITING FREOUENCY $=5 \mathrm{MC}$

- LIMITING FREQUENCY $=7 \mathrm{MC}$.

Fig. 10. WHITE SANDS, NEW MEXICO AUGUST 1949
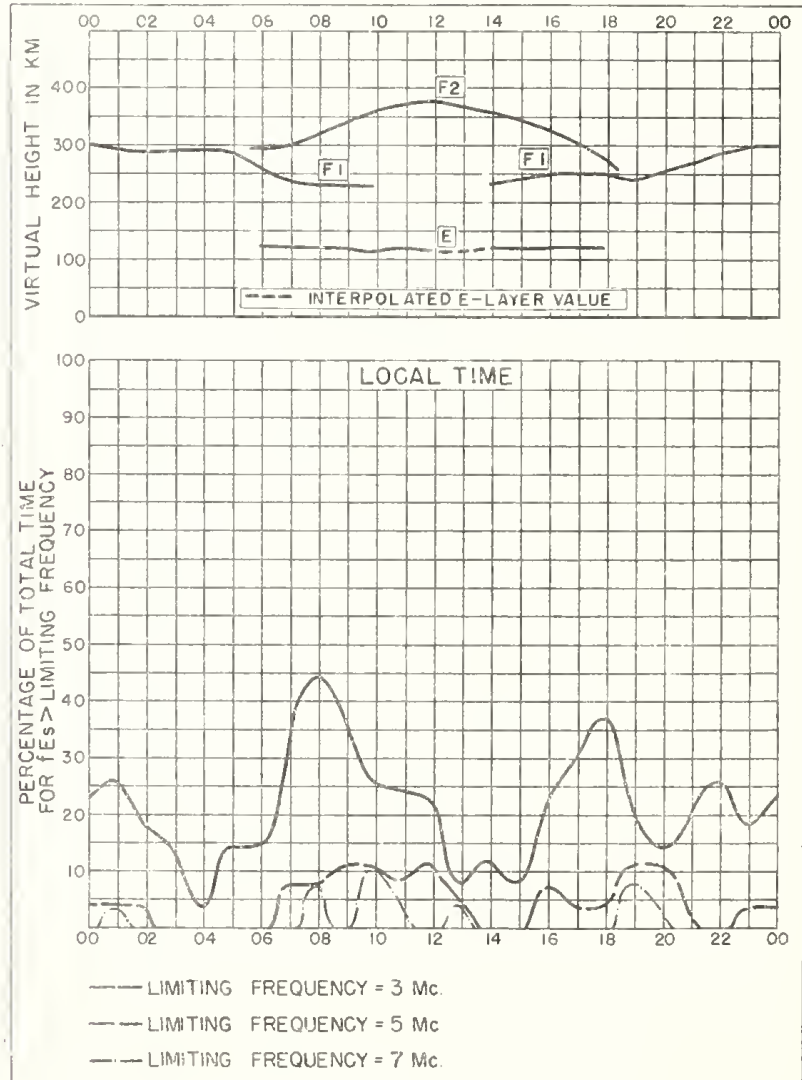

Fig 12. BATON ROUGE, LOUISIANA

AUGUST 1949 


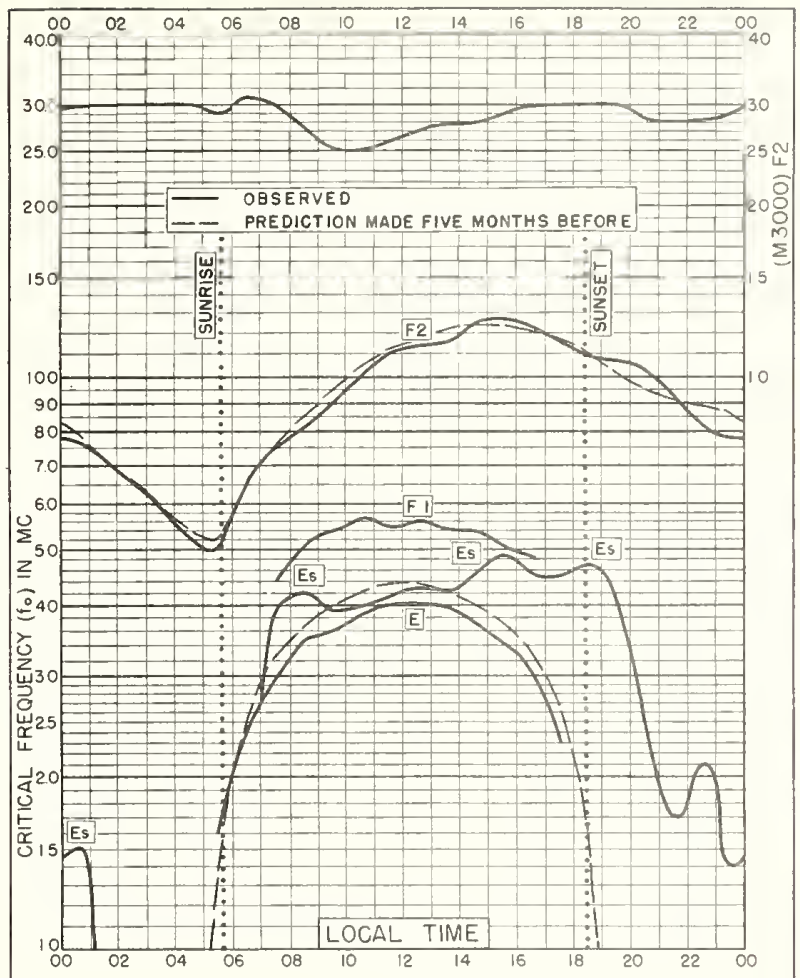

Fig. 13. MAUI, HAWAII

$20.8^{\circ} \mathrm{N}, 156.5^{\circ} \mathrm{W}$

AUGUST 1949

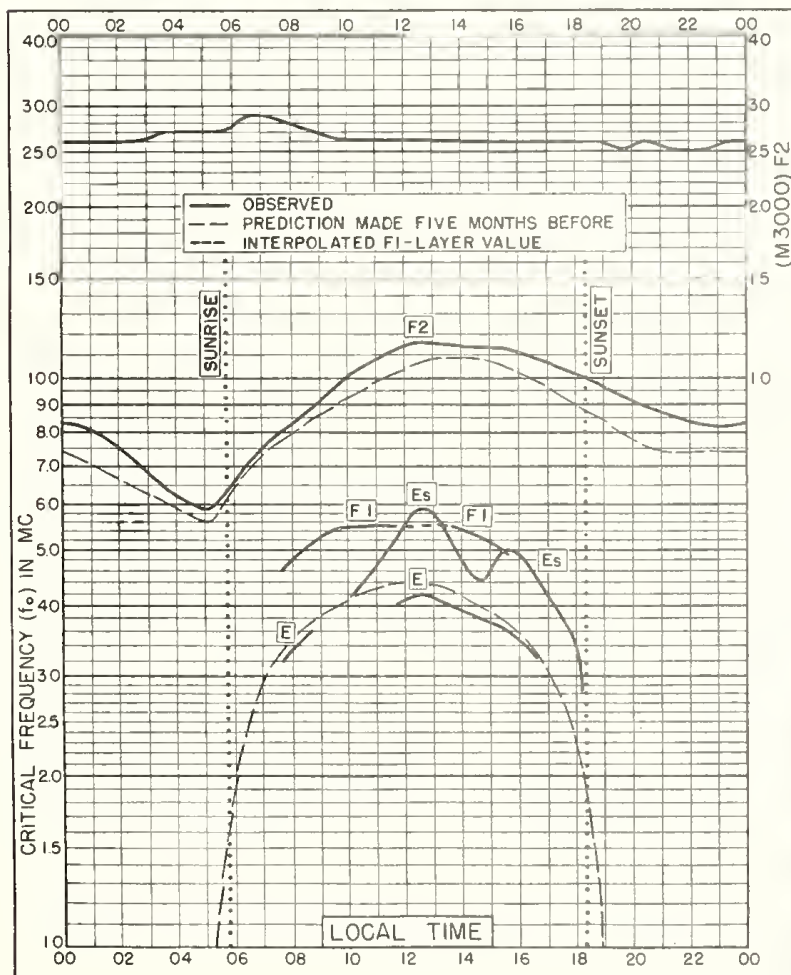

Fig. 15. SAN JUAN, PUERTO RICO

$18.4^{\circ} \mathrm{N}, 66.1^{\circ} \mathrm{W}$

AUGUST 1949
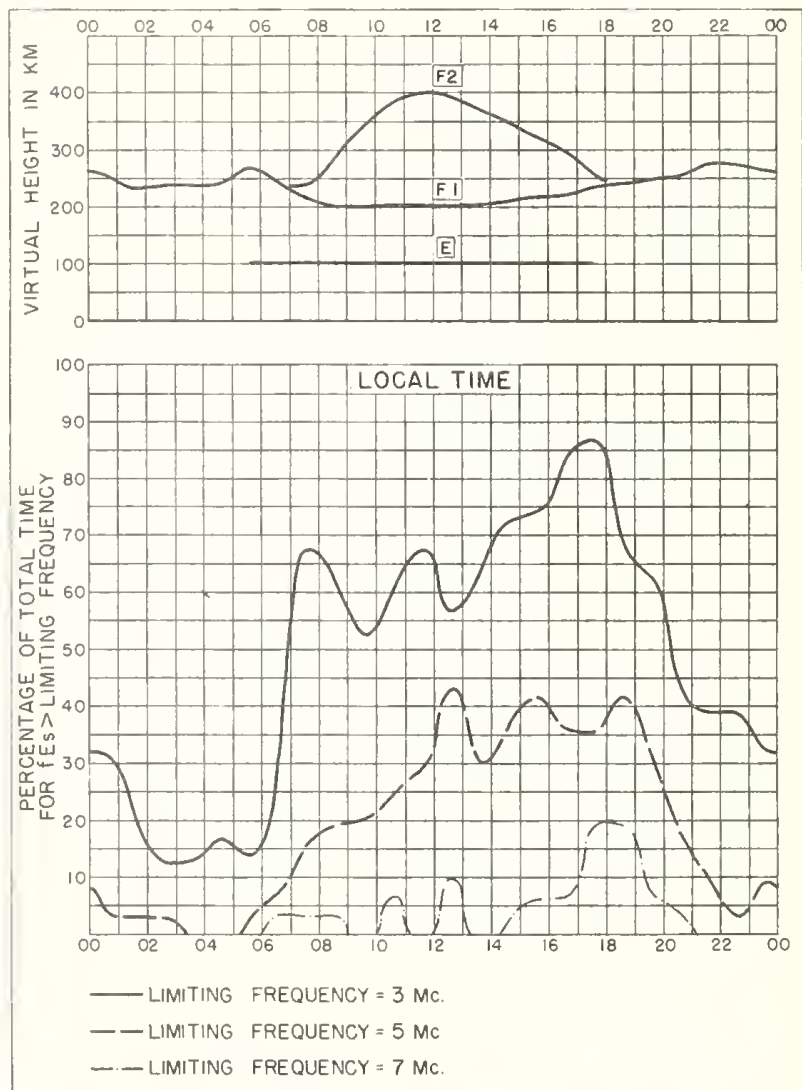

Fig. 14. MAUI, HAWAII

AUGUST 1949

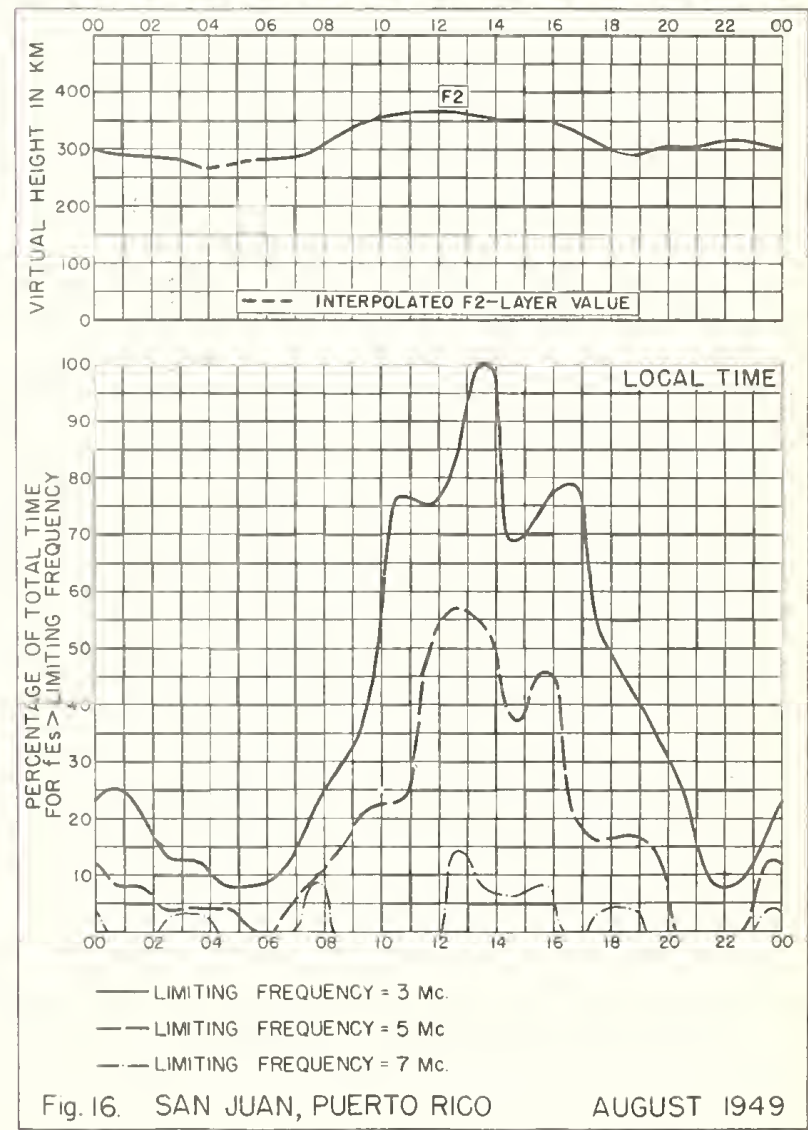




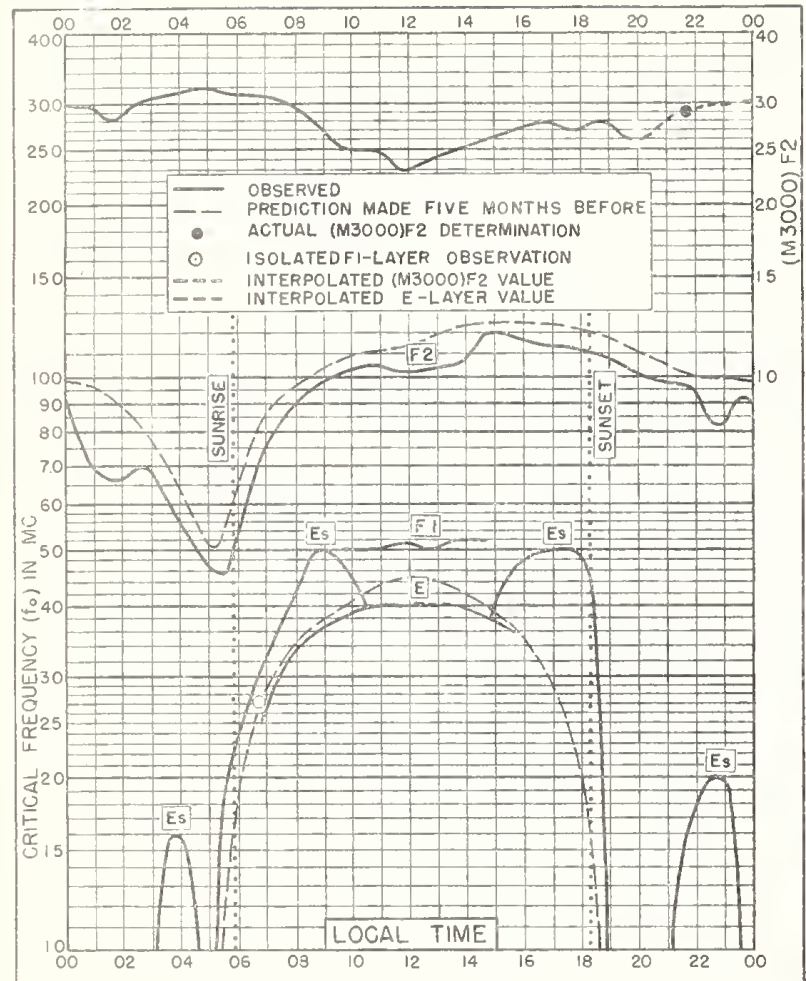

Fig. 17 GUAM I.

$3.6^{\circ} \mathrm{N}, 144.9^{\circ} \mathrm{E} \quad$ AUGUST 1949

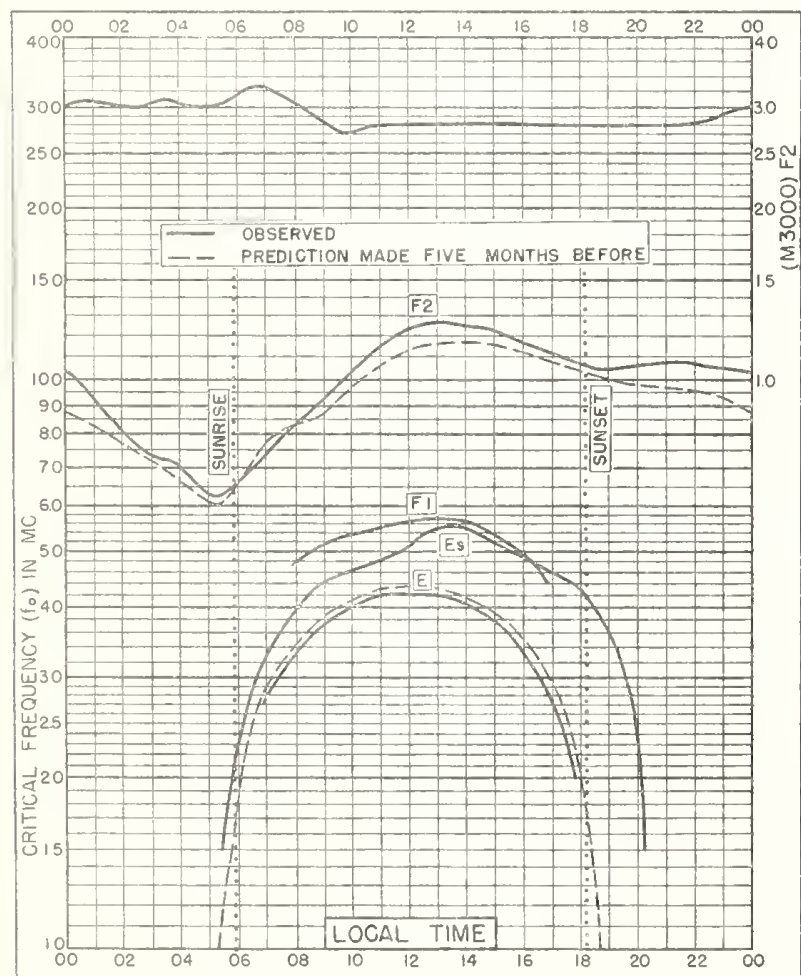

FIC. 19 TRINIDAD, BRIT. WEST INDIES

$10.6^{\circ} \mathrm{N}, 612^{\circ} \mathrm{W}$

AUGUST 1949
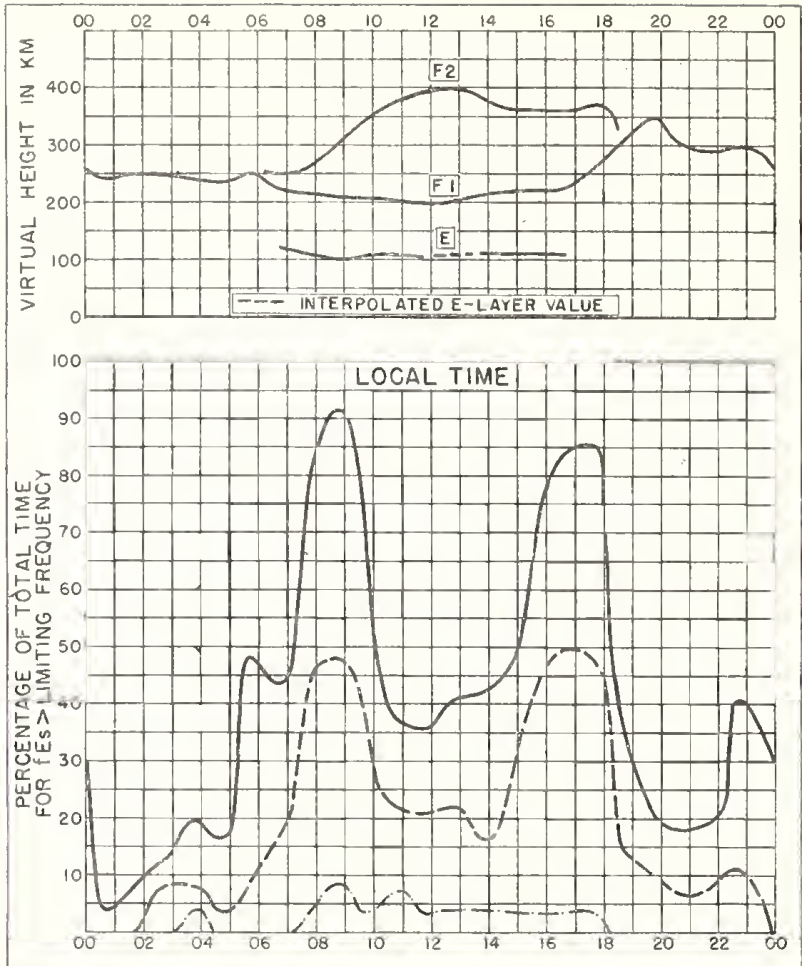

- LIMITING FREQUENCY $=3 \mathrm{MC}$.
- LIMITING FREQUENCY $=5 \mathrm{MC}$.
- LIMITING FREQUENCY $=7 \mathrm{MC}$.

Fig 18. GUAM I.

AUGUST 1949
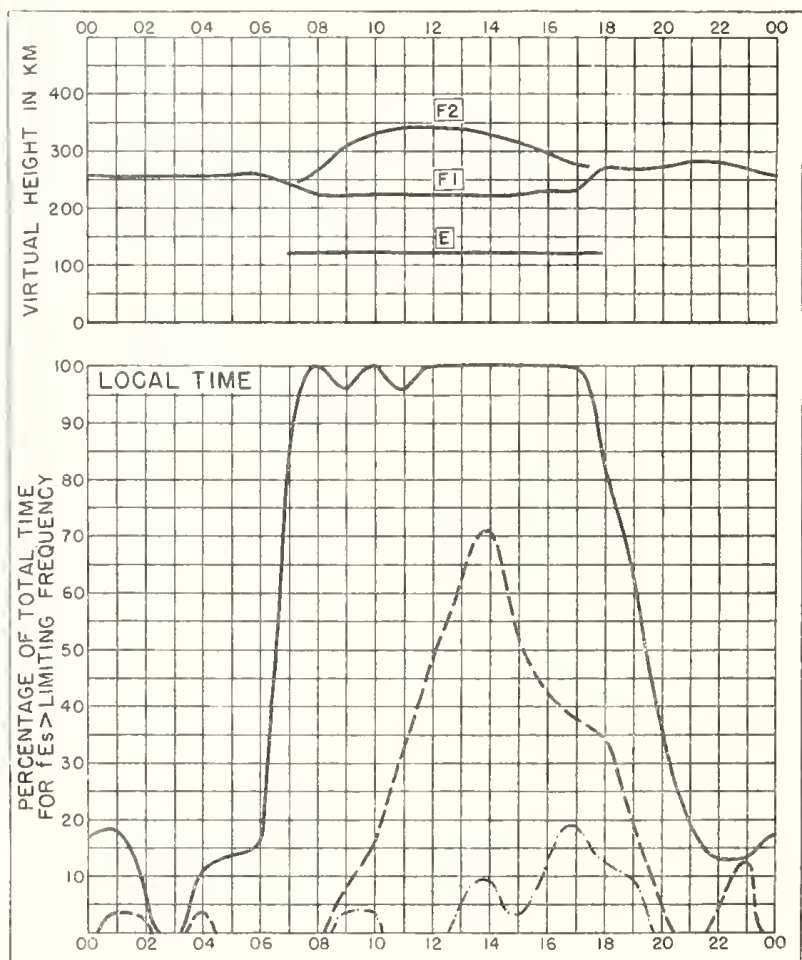

\section{- LIMITING FREQUENCY $=3 \mathrm{MC}$. \\ - LIMITING FREQUENCY $=5 \mathrm{MC}$ \\ - LIMITING FREQUENCY $=7 \mathrm{MC}$.}

Fig 2O. TRINIDAO, BRIT WEST INDIES AUGUST 1949 


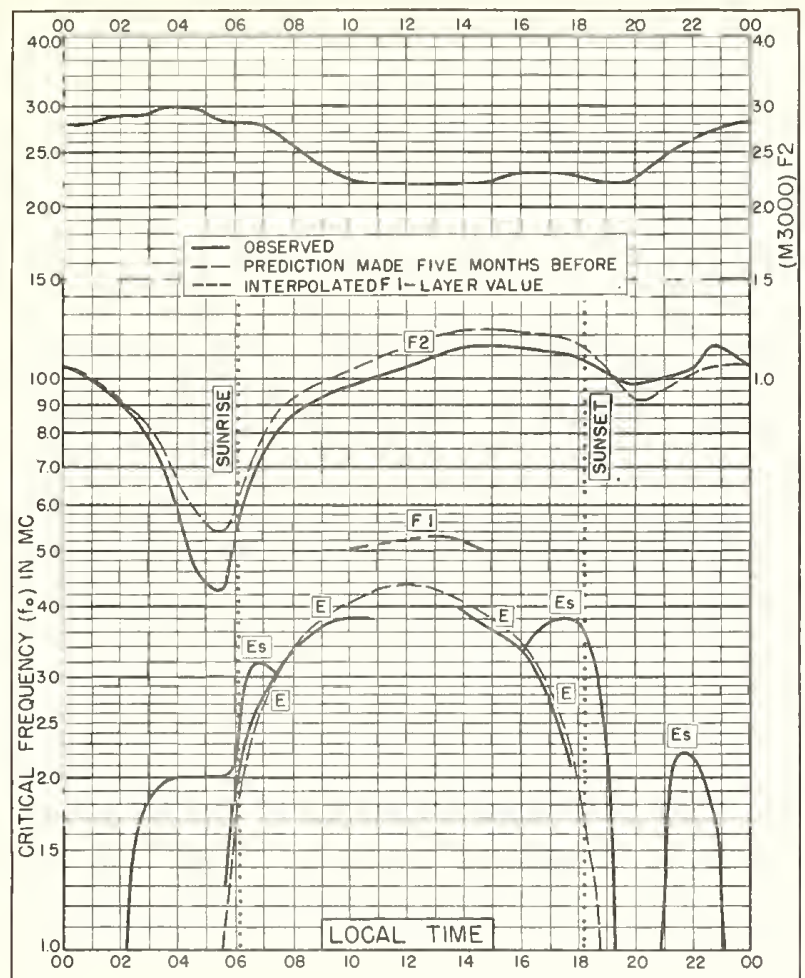

Fig. 21 PALMYRA I

$5.9^{\circ} \mathrm{N}, 162.1^{\circ} \mathrm{W}$

AUGUST 1949

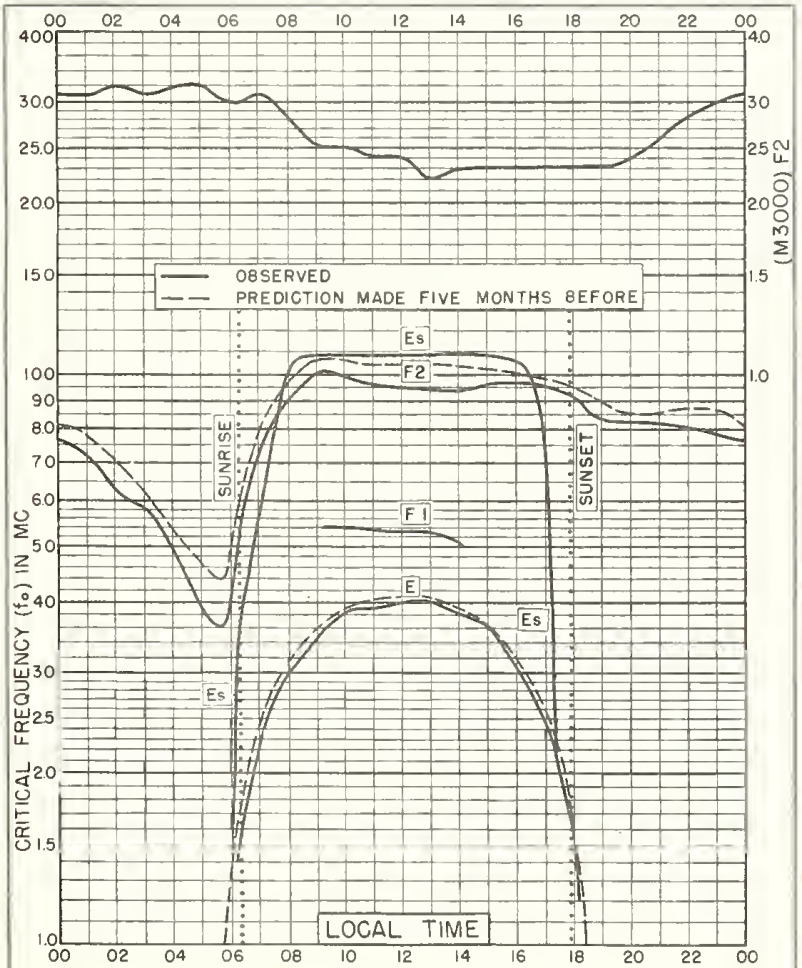

Fig 23. HUANCAYO, PERU

$12.0^{\circ} \mathrm{S}, 75.3^{\circ} \mathrm{W}$

AUGUST 1949
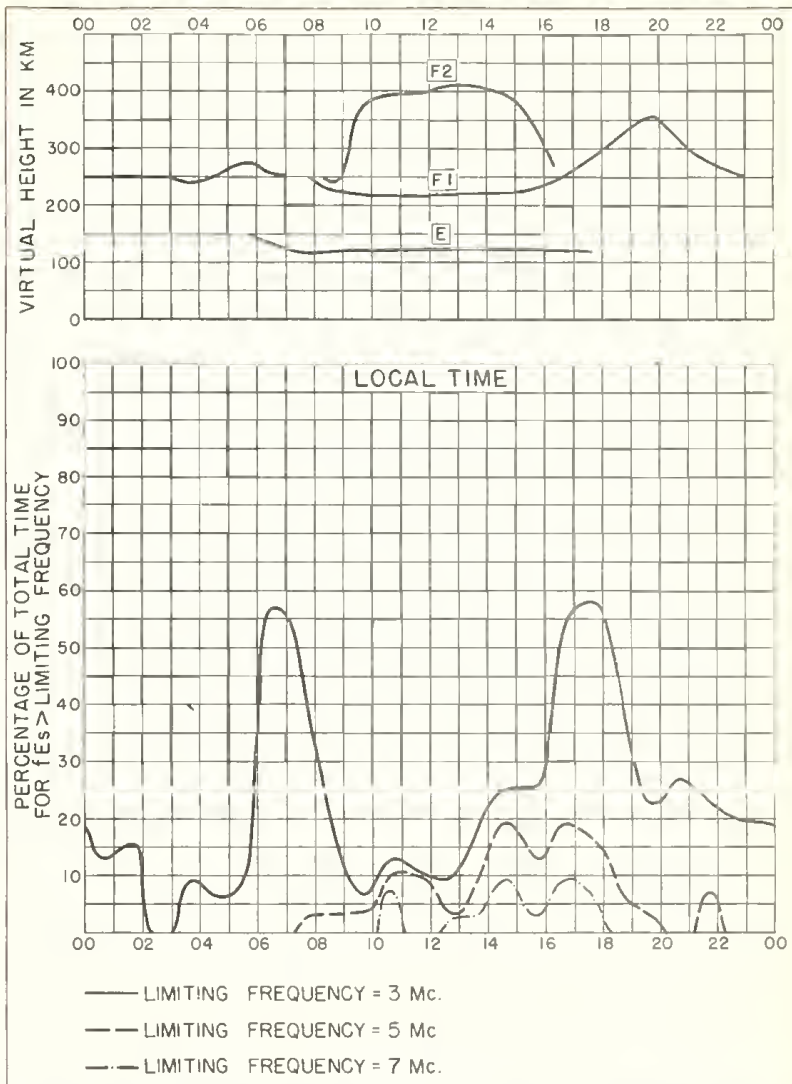

Fig 22. PALMYRA I

AUGUST 1949
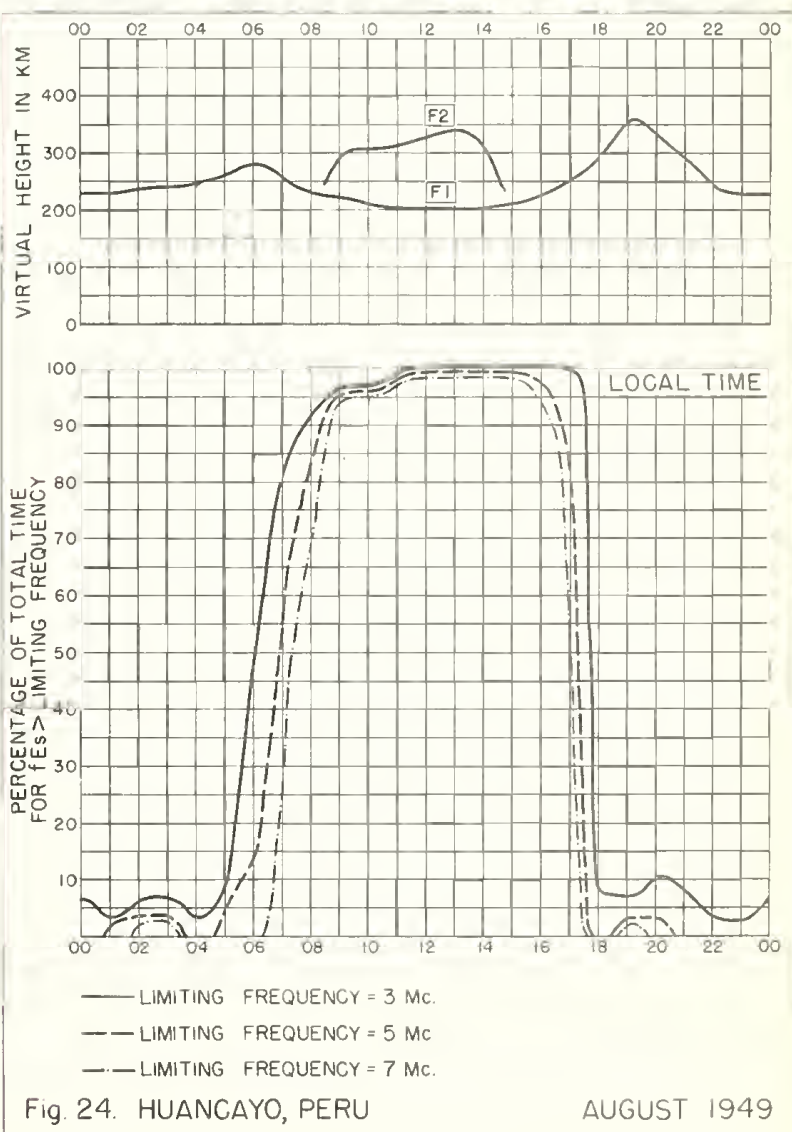


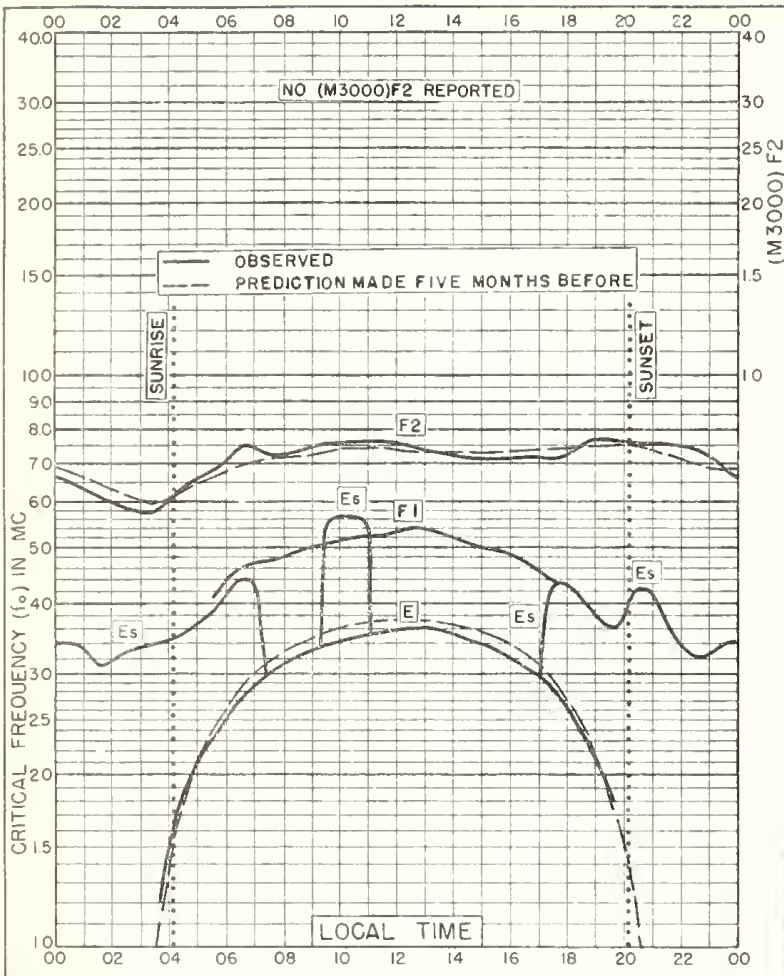

Fig. 25. LINDAU/HARZ, GERMANY $51.6^{\circ} \mathrm{N}, 10.1^{\circ} \mathrm{E}$

JULY 1949

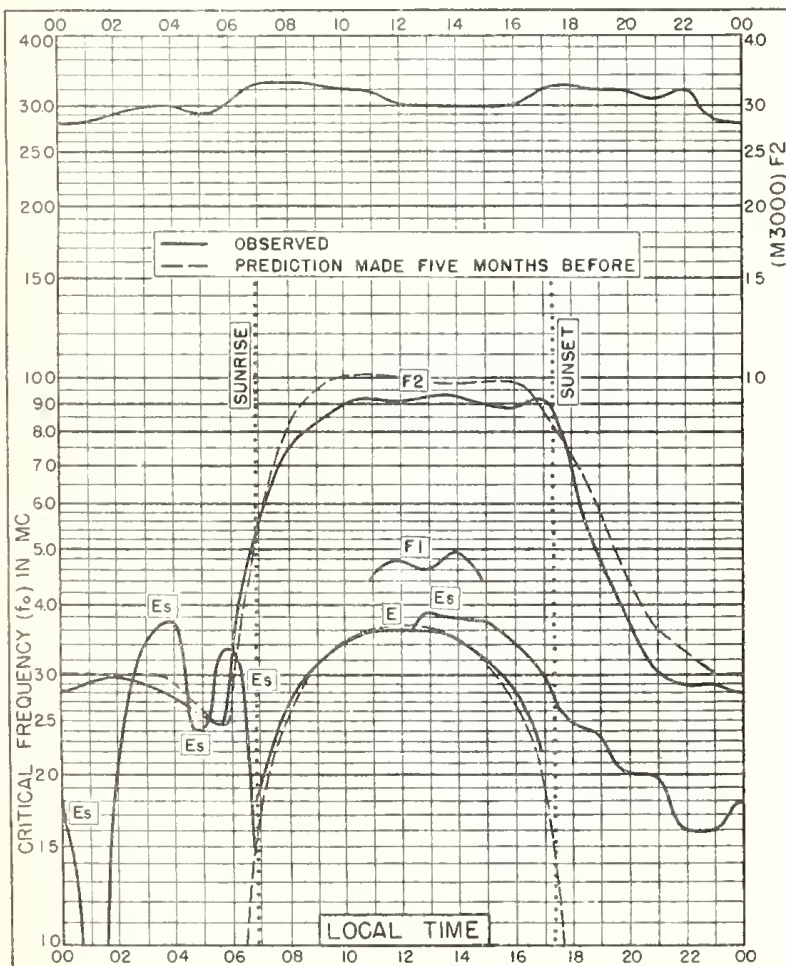

Fig 27 JOHANNESBURG, U. OF S AFRICA $262^{\circ} \mathrm{S}, 280^{\circ} \mathrm{E}$

JULY 1949
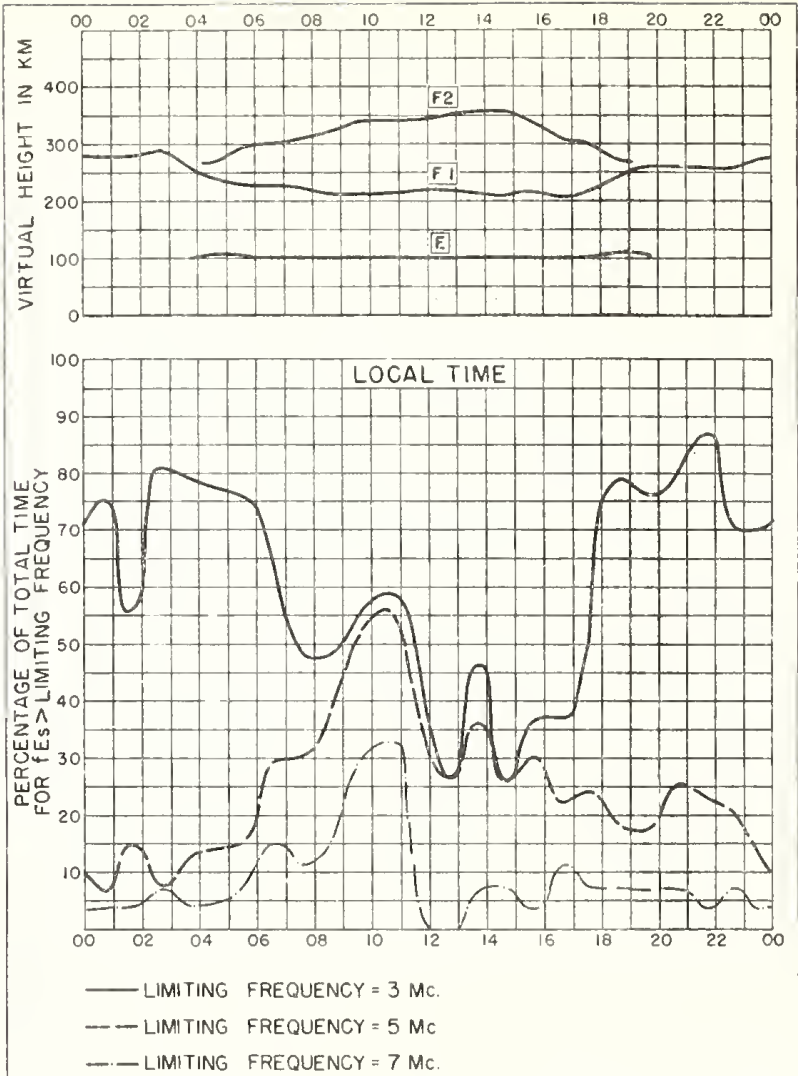

Fig. 26. LINDAU/HARZ, GERMANY

JULY 1949
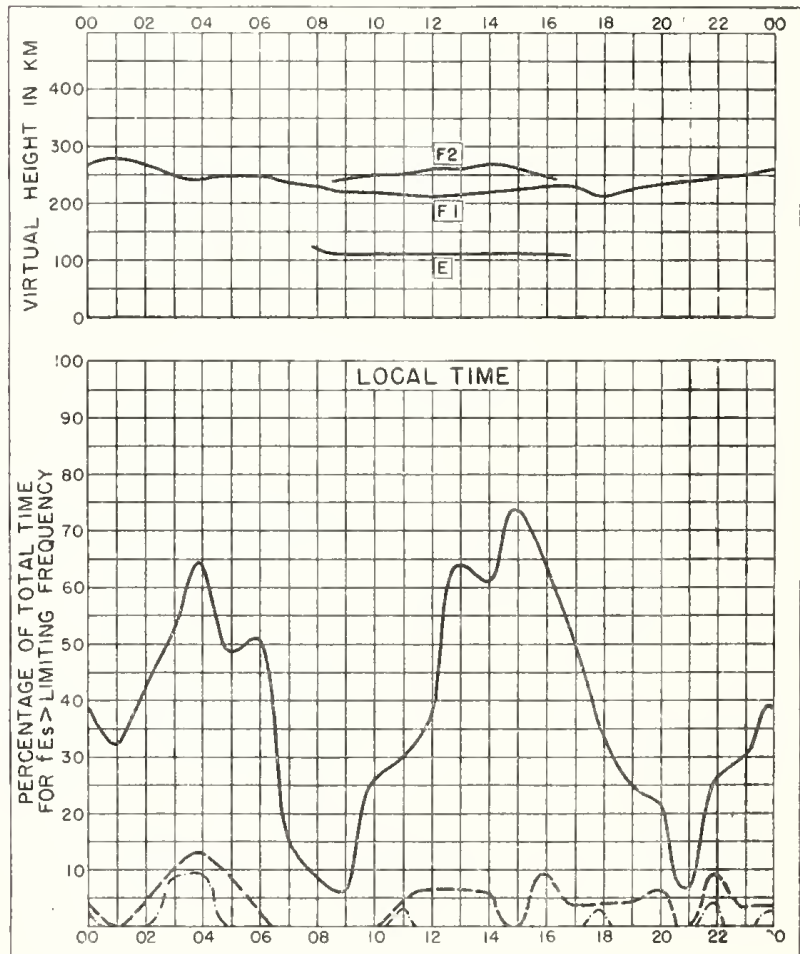

- LIMITING FREQUENCY $=3 \mathrm{MC}$.

- LIMITING FREQUENCY $=5 \mathrm{MC}$

- LIMITING FREQUENCY $=7 \mathrm{Mc}$.

Fig 28 JOHANNESBURG, U. OF S. AFRICA JULY 1949 


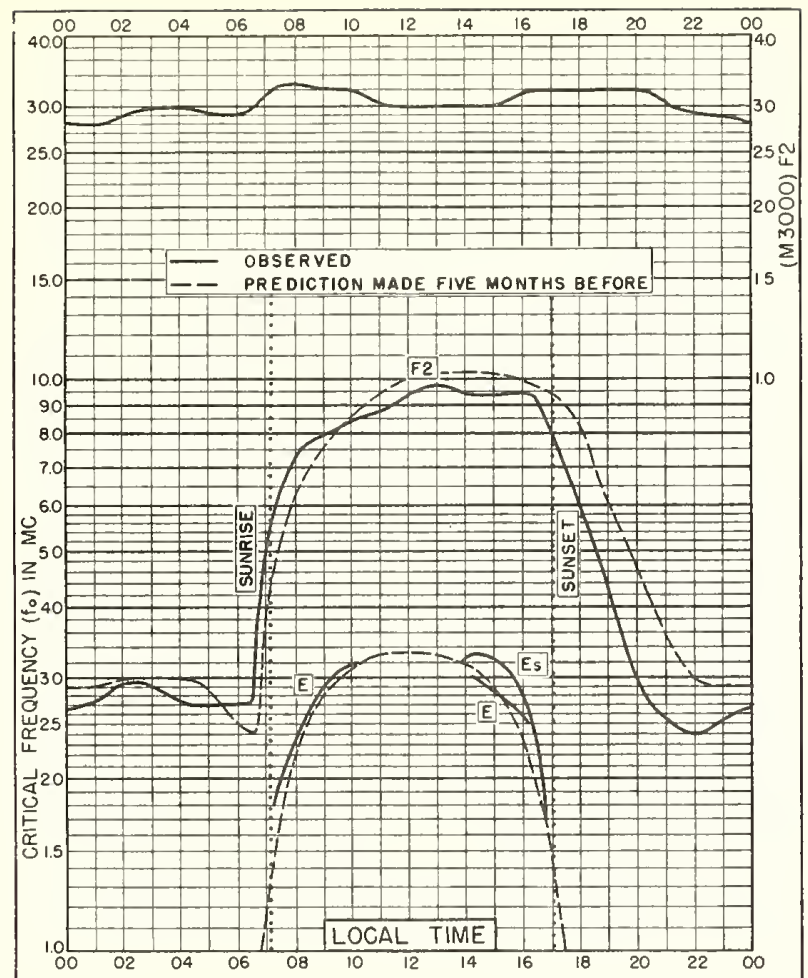

Fig 29. CAPETOWN, U. OF S. AFRICA $34.2^{\circ} \mathrm{S}, 18.3^{\circ} \mathrm{E}$

JULY 1949

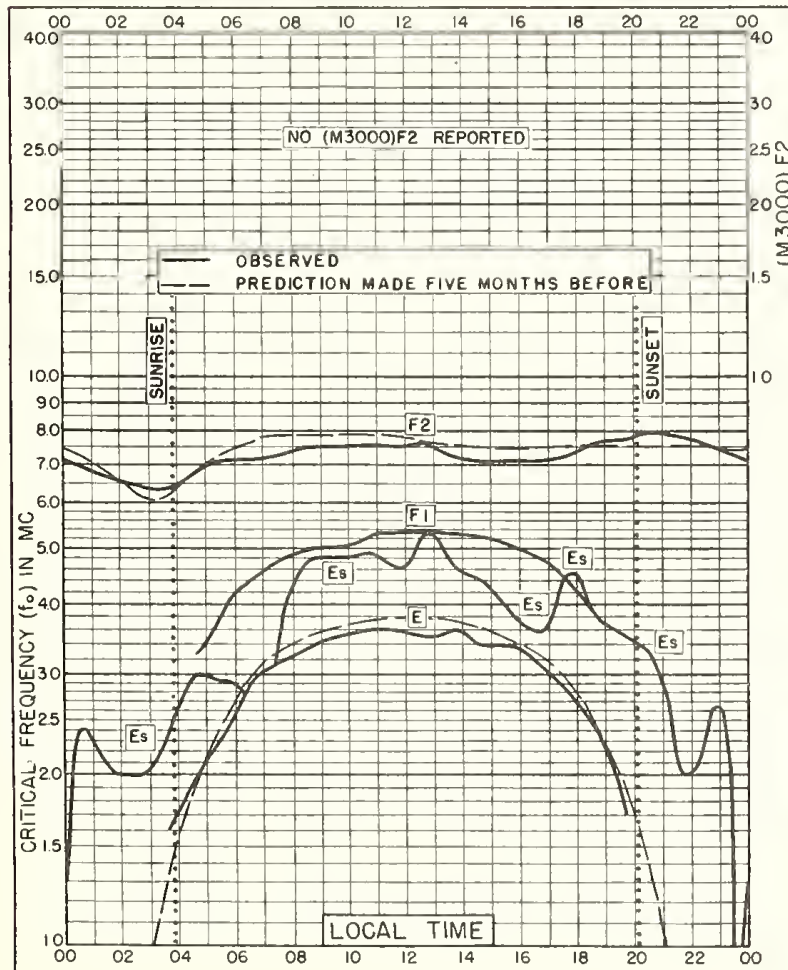

Fig. 31. LINDAU/HARZ, GERMANY 51. $6^{\circ} \mathrm{N}, 10.1^{\circ} \mathrm{E}$
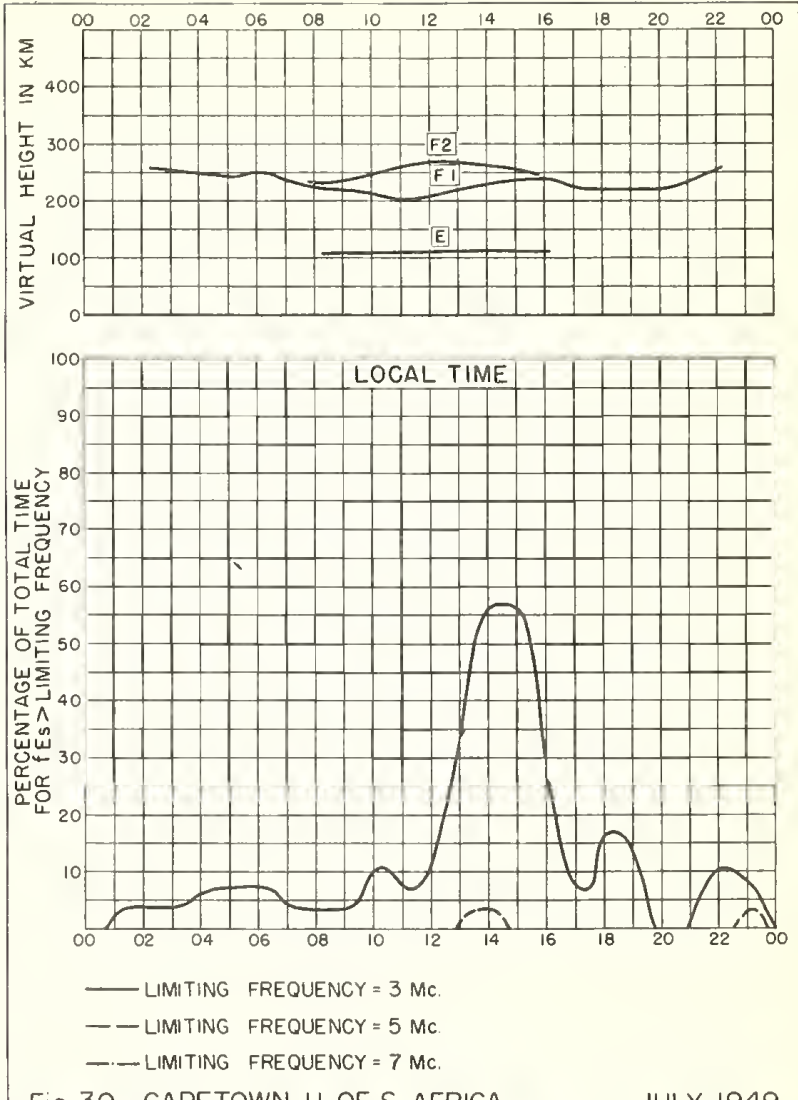

Fig. 30 CAPETOWN, U. OF S. AFRICA

JULY 1949

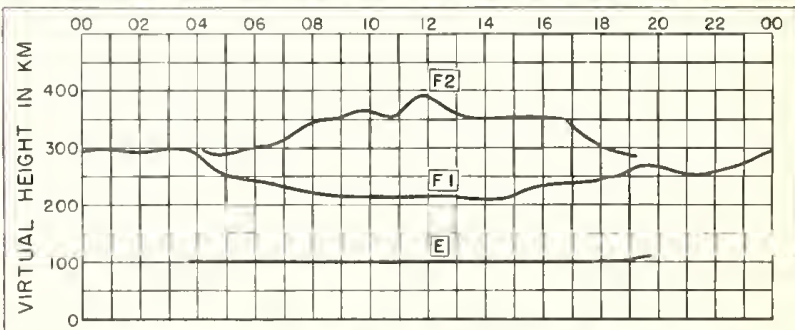

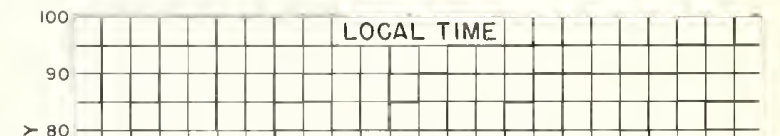

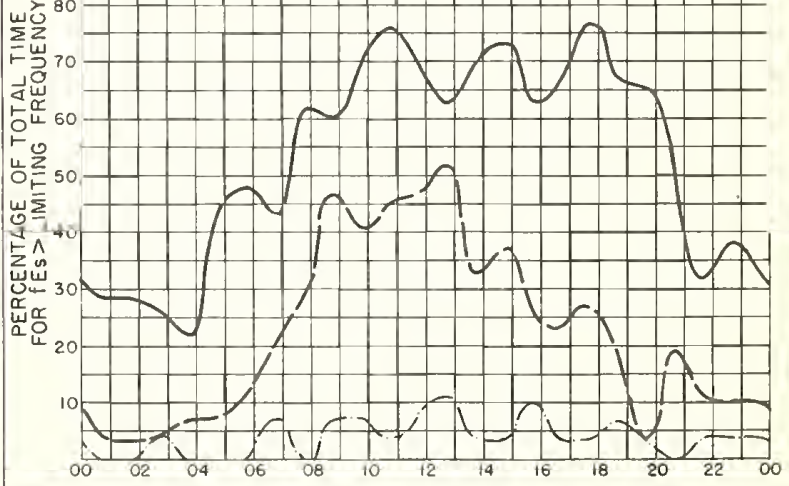

- LIMITING FREQUENCY $=3 \mathrm{MC}$.
- - LIMITING FREQUENCY $=5 \mathrm{MC}$
- LIMITING FREQUENCY $=7 \mathrm{MC}$.

Fig. 32. LINDAU/HARZ, GERMANY JUNE 1949 
50

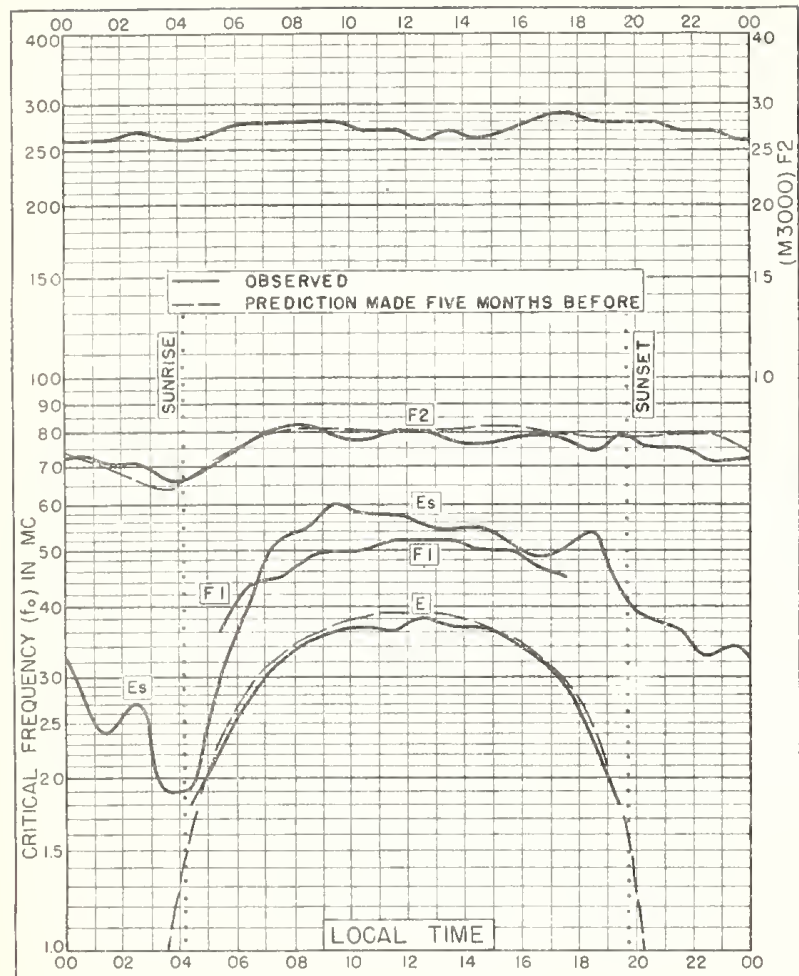

Fig. 33 WAKKANAI, JAPAN $45.4^{\circ} \mathrm{N}, 141.7^{\circ} \mathrm{E}$

JUNE 1949

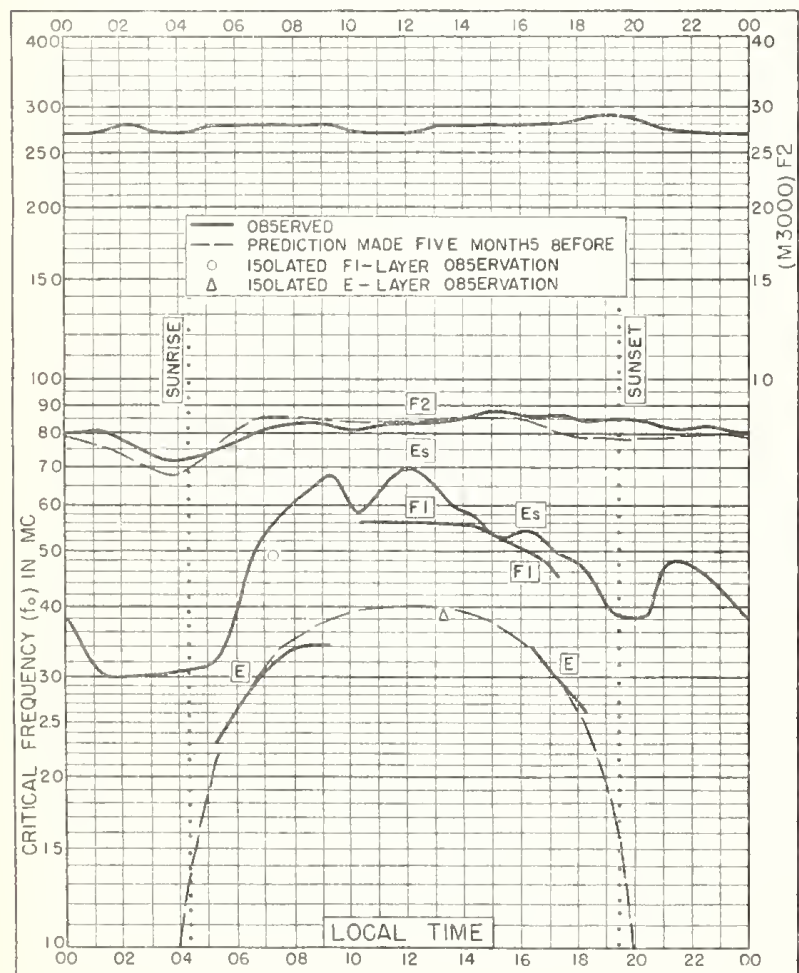

Fig. 35 FUKAURA, JAPAN $40.6^{\circ} \mathrm{N}, 139.9^{\circ} \mathrm{E}$
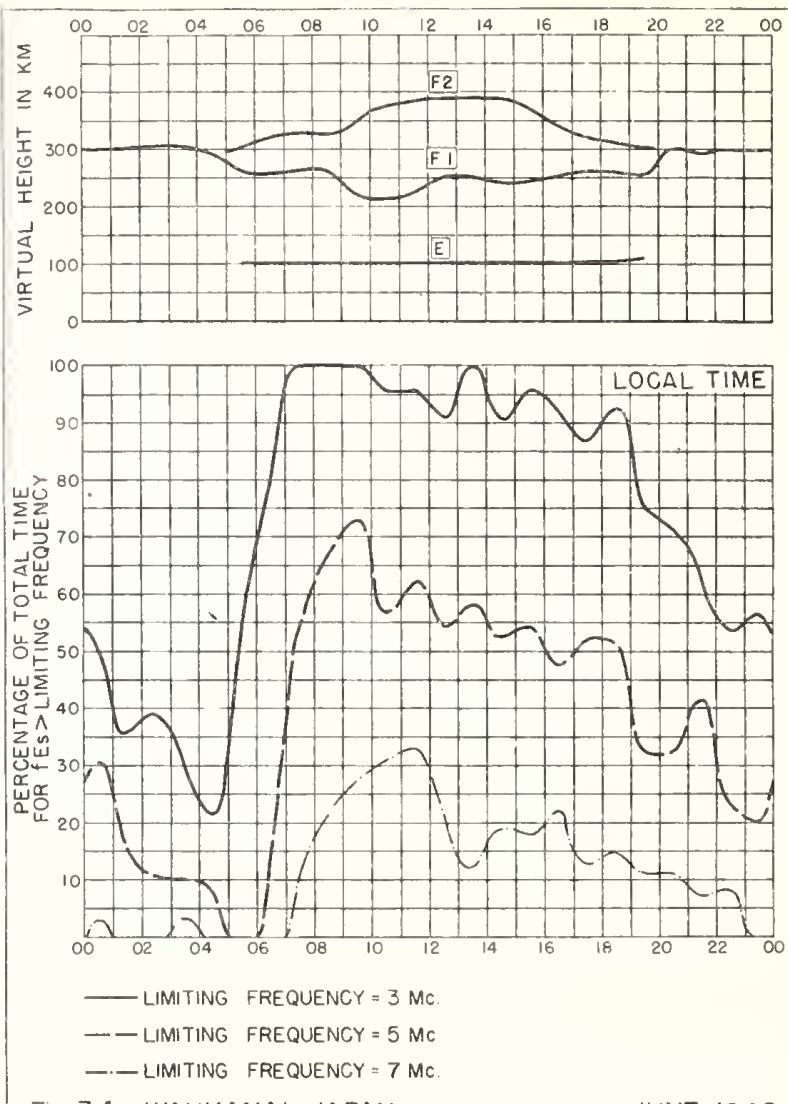

Fig. 34 WAKKANAI, JAPAN

JUNE 1949
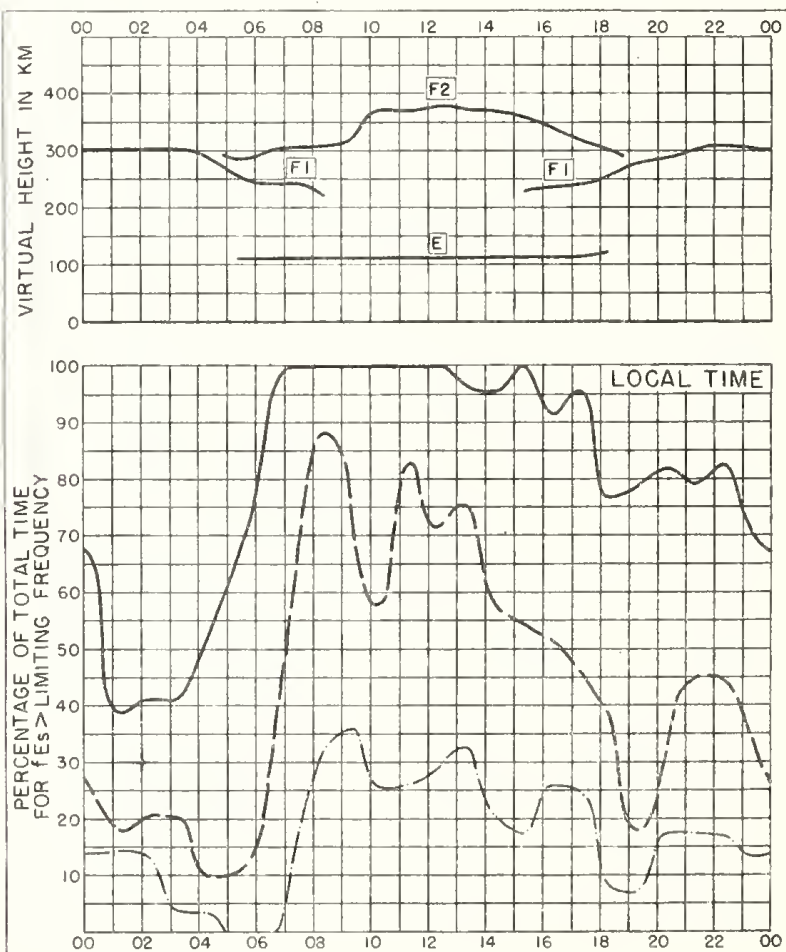

\footnotetext{
LIMITING FREQUENCY $=3 \mathrm{MC}$.

- LIMITING FREQUENCY $=5 \mathrm{MC}$

- LIMITING FREQUENCY $=7 \mathrm{MC}$.
}

Fig. 36. FUKAURA, JAPAN 


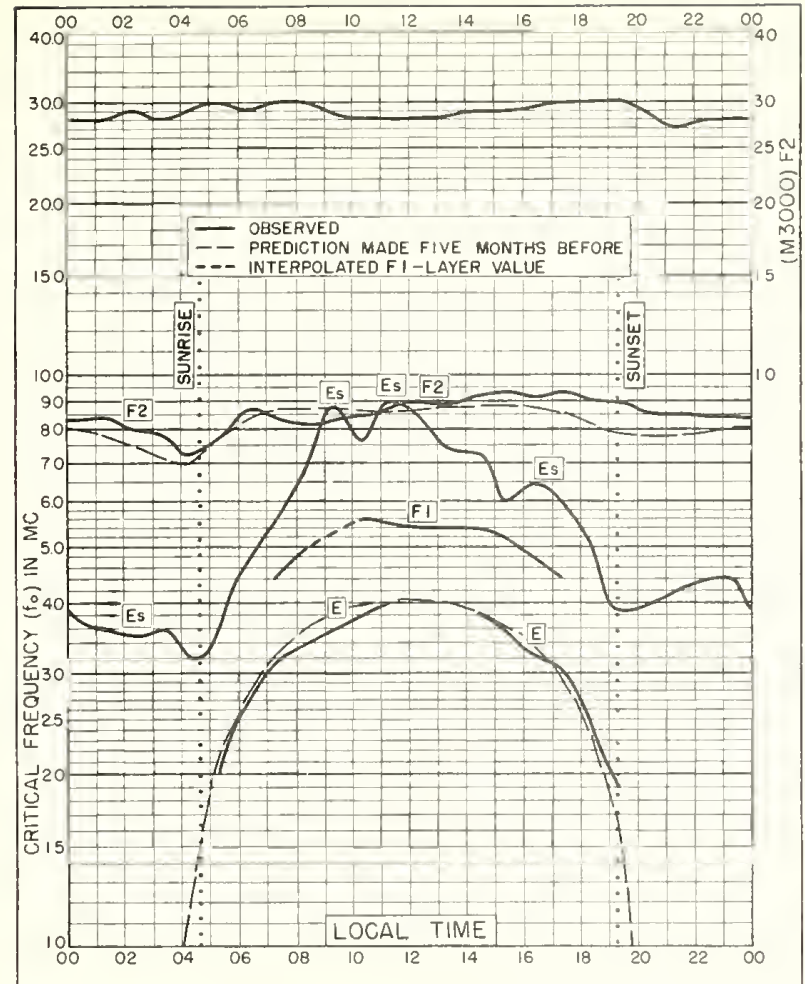

Fig. 37. SHIBATA, JAPAN

$37.9^{\circ} \mathrm{N}, 139.3^{\circ} \mathrm{E}$

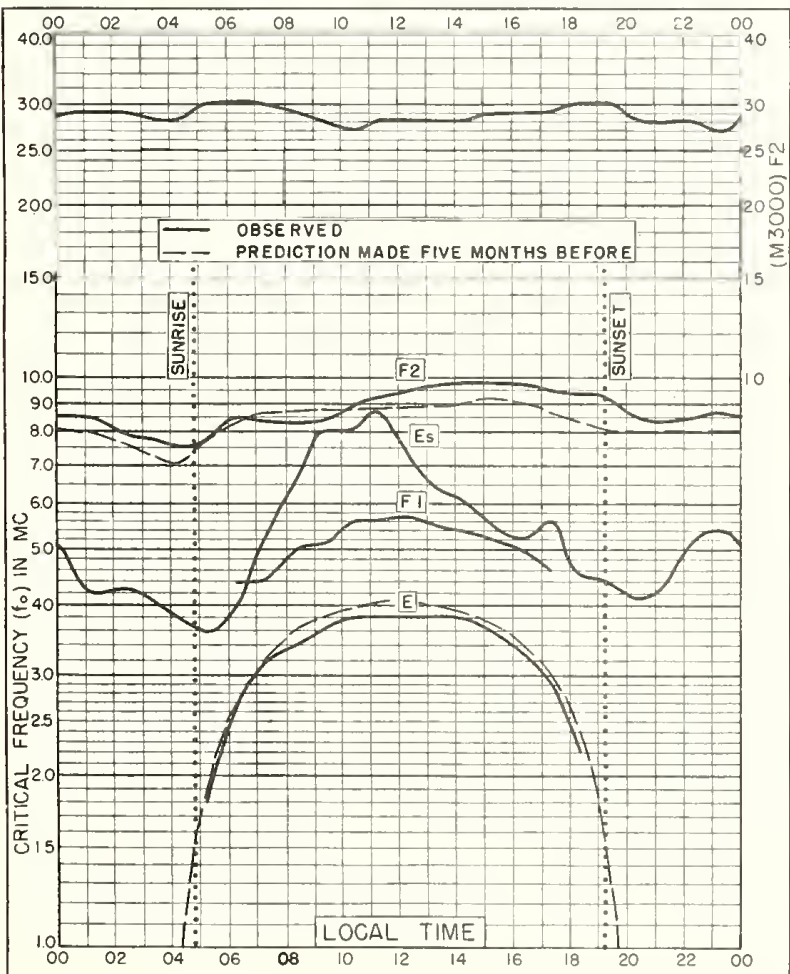

Fig. 39 TOKYO, JAPAN

$35.7^{\circ} \mathrm{N}, 139.5^{\circ} \mathrm{E}$
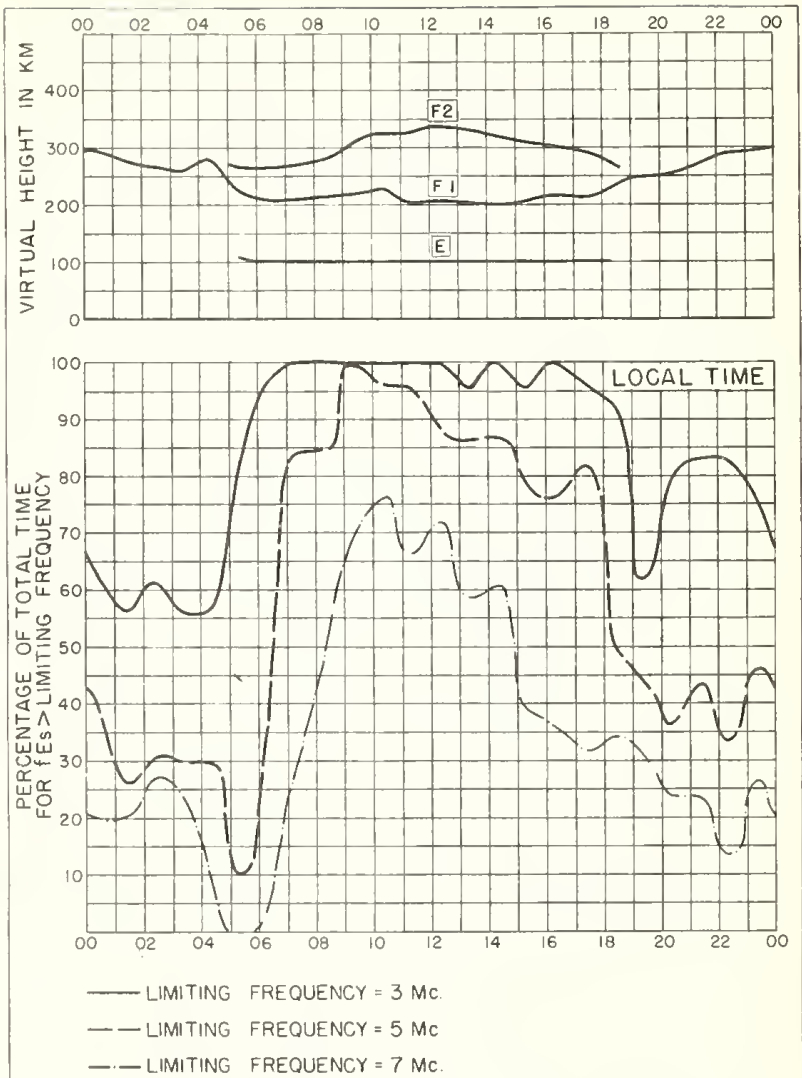

Fig. 38 SHIBATA, JAPAN

JUNE 1949
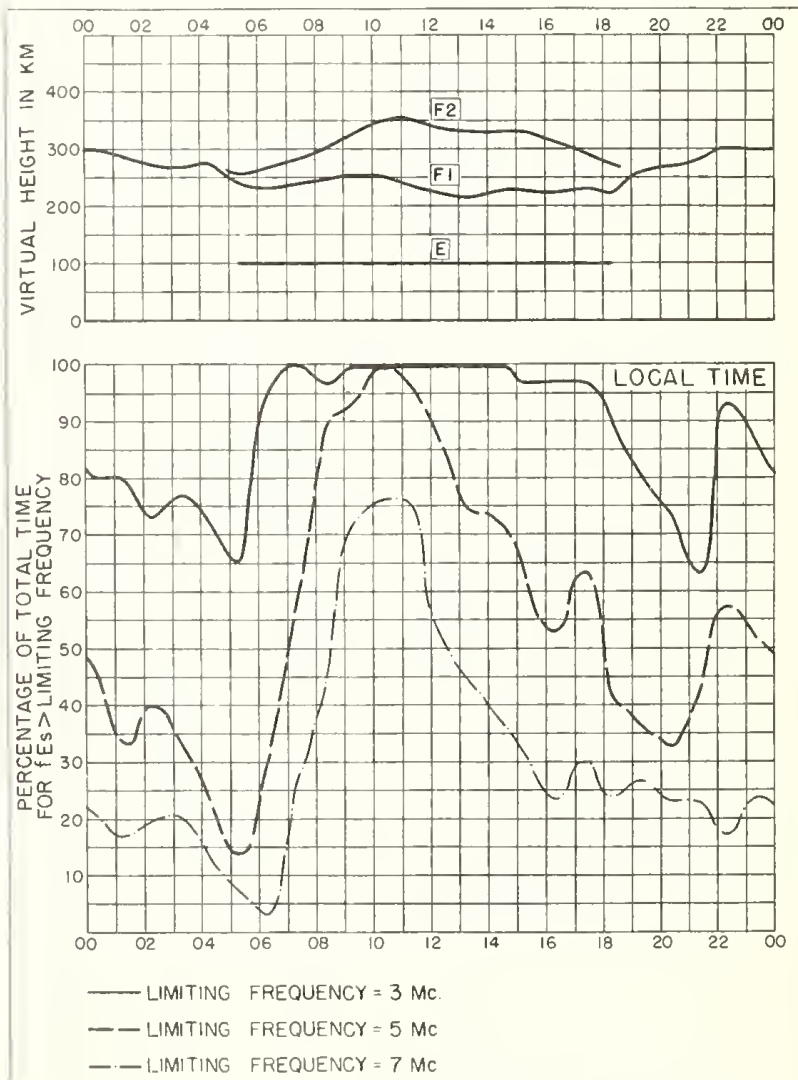

Fig. 40. TOKYO, JAPAN

JUNE 1949 


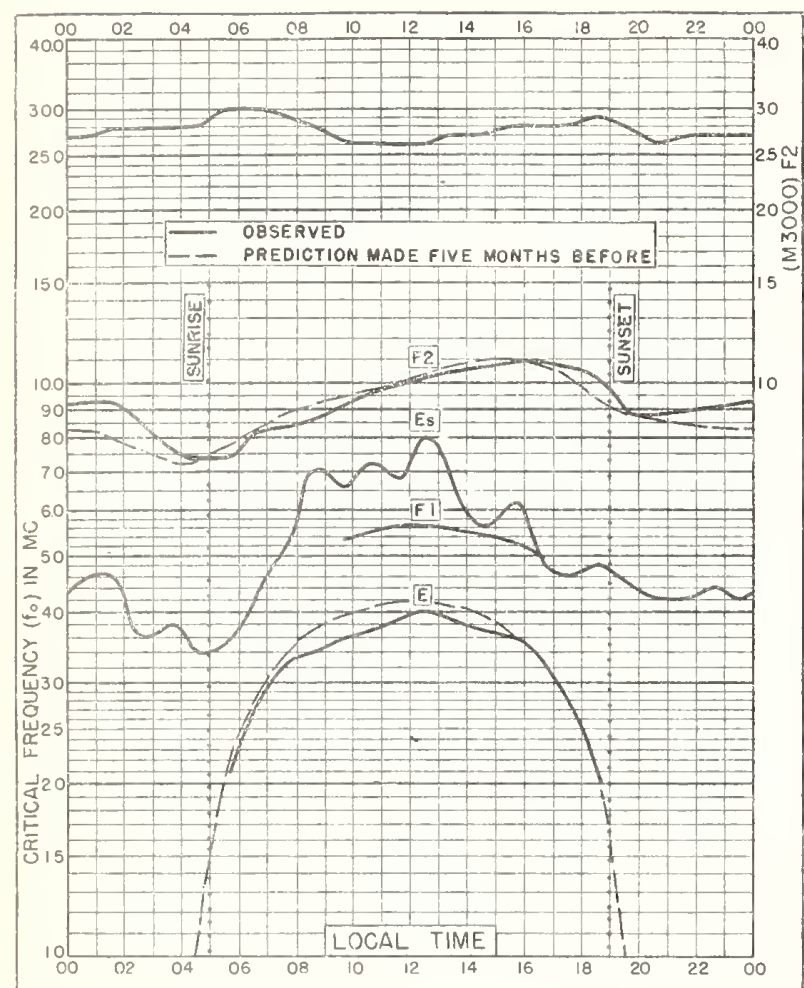

Fig. 41. YAMAKAWA, JAPAN $31.2^{\circ} \mathrm{N}, 130.6^{\circ} \mathrm{E}$ JUNE 1949

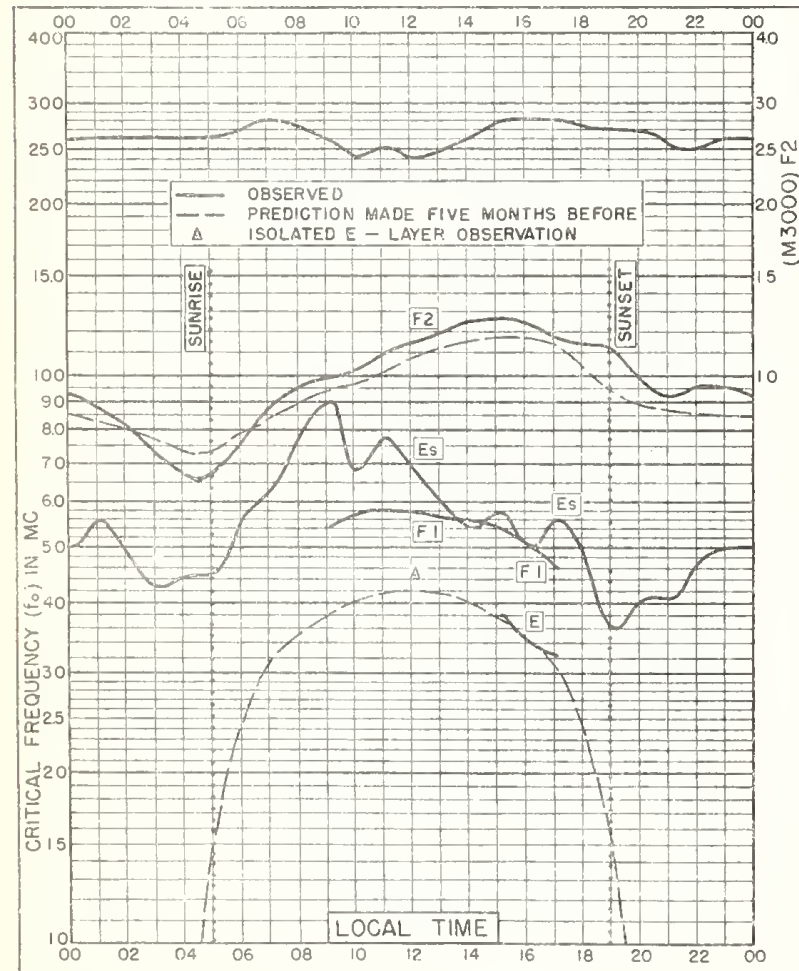

Fig. 43 CHIUNGKING, CHINA $29.4^{\circ} \mathrm{N}, 106.8^{\circ} \mathrm{E}$
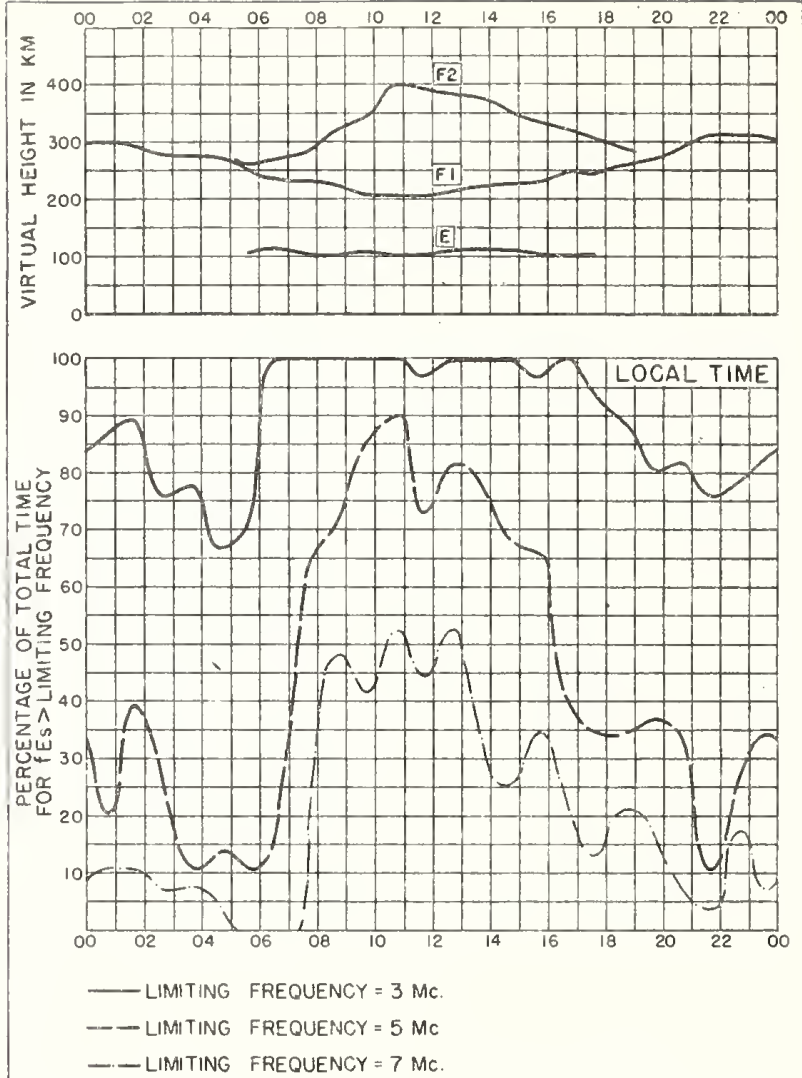

Fig. 42 YAMAKAWA, JAPAN

JUNE 1949
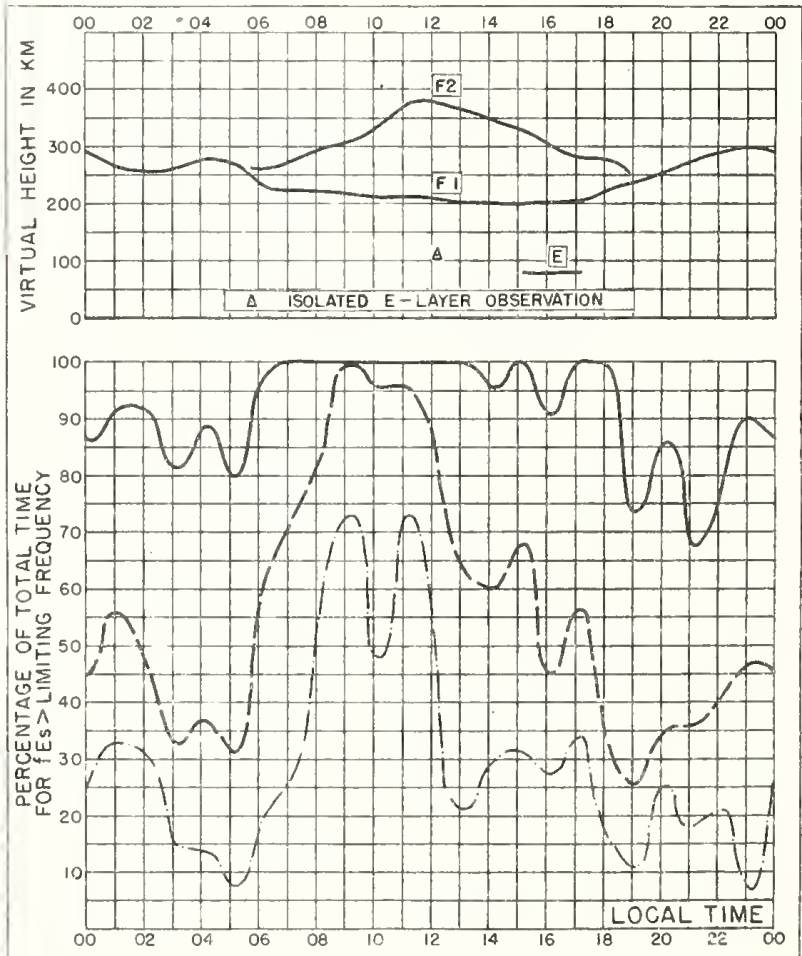

\footnotetext{
LIMITING FREQUENCY $=3 \mathrm{MC}$

- LIMITING FREQUENCY $=5 \mathrm{MC}$

- LIMITING FREQUENCY $=7 \mathrm{MC}$
}

Fig. 44 CHUNGKING, CHINA JUNE 1949 


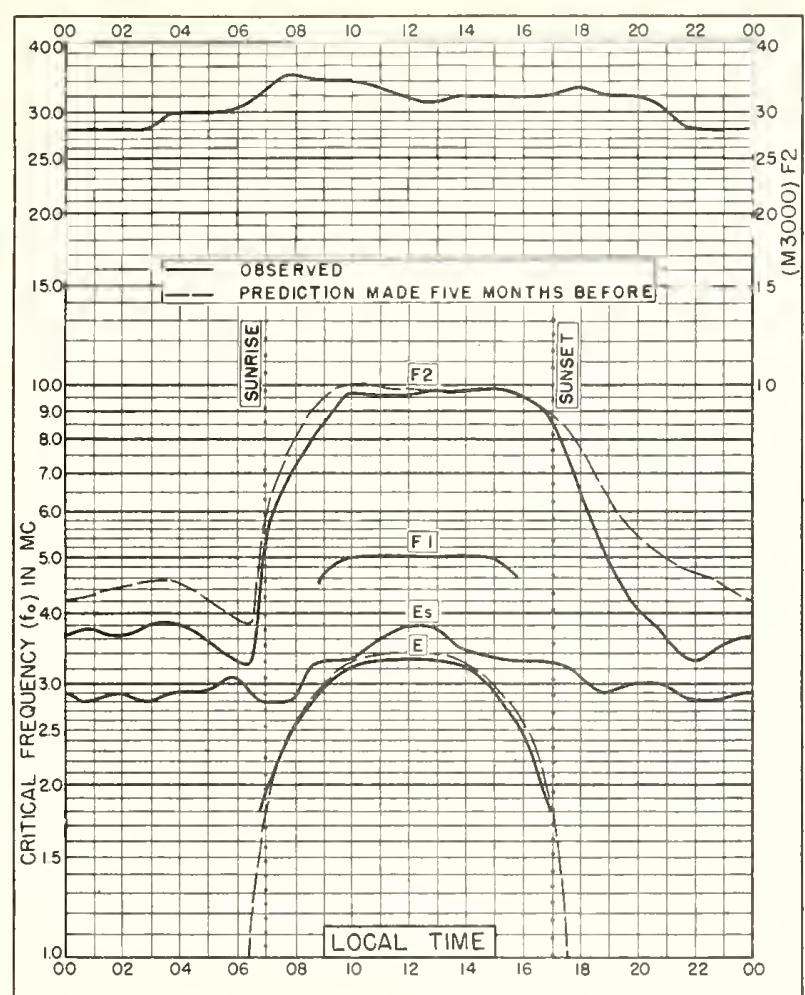

Fig 45. WATHEROO, W AUSTRALIA $30.3^{\circ} \mathrm{S}, 115.9^{\circ} \mathrm{E}$

JUNE 1949

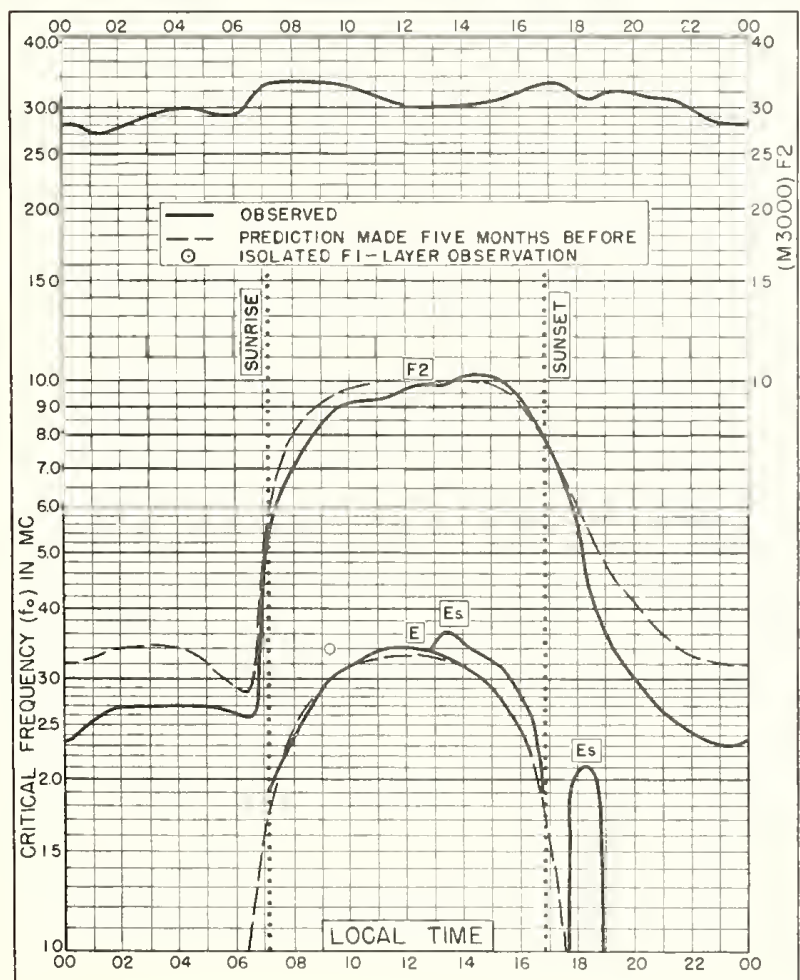

Fig. 47. CAPETOWN, U. OF S AFRICA $34.2^{\circ} \mathrm{S}, 18.3^{\circ} \mathrm{E}$ JUNE 1949
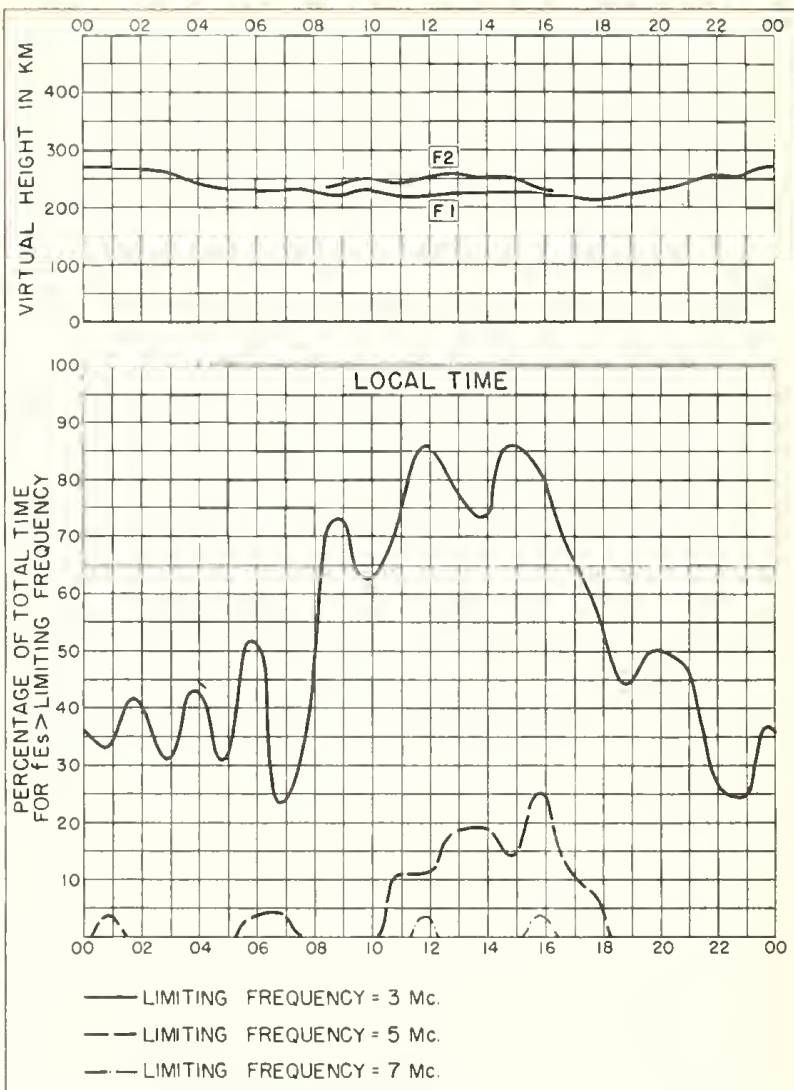

Fig 46. WATHEROO, W AUSTRALIA

JUNE 1949
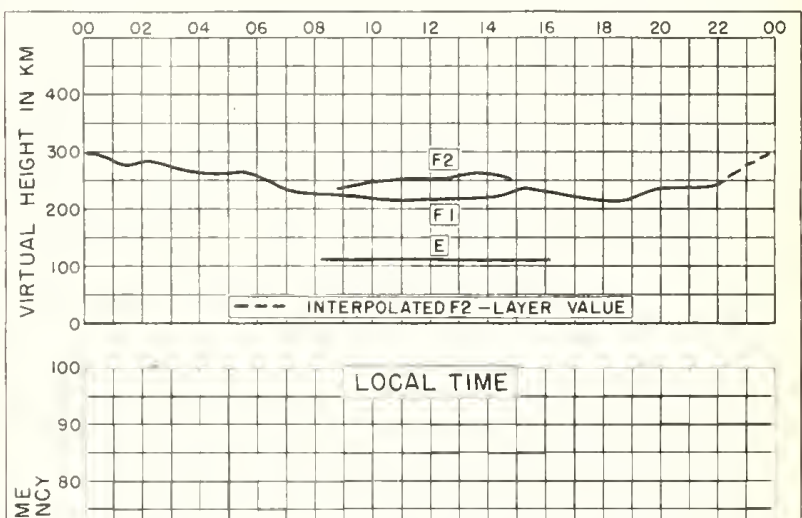

告

崖7

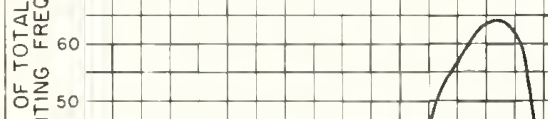

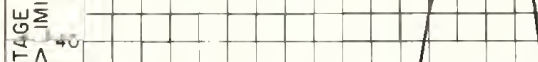

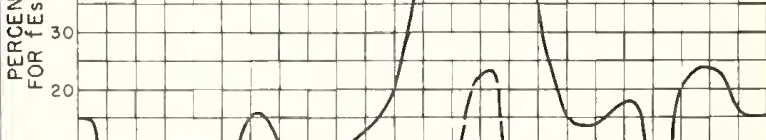

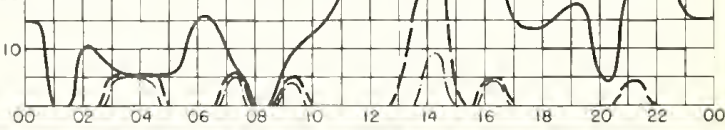

- LIMITING FREQUENCY $=3 \mathrm{MC}$.
- LIMITING FREQUENCY $=5 \mathrm{MC}$
- - LIMITING FREQUENCY $=7 \mathrm{MC}$

Fig. 48. CAPETOWN, U OF S. AFRICA

JUNE 1949 


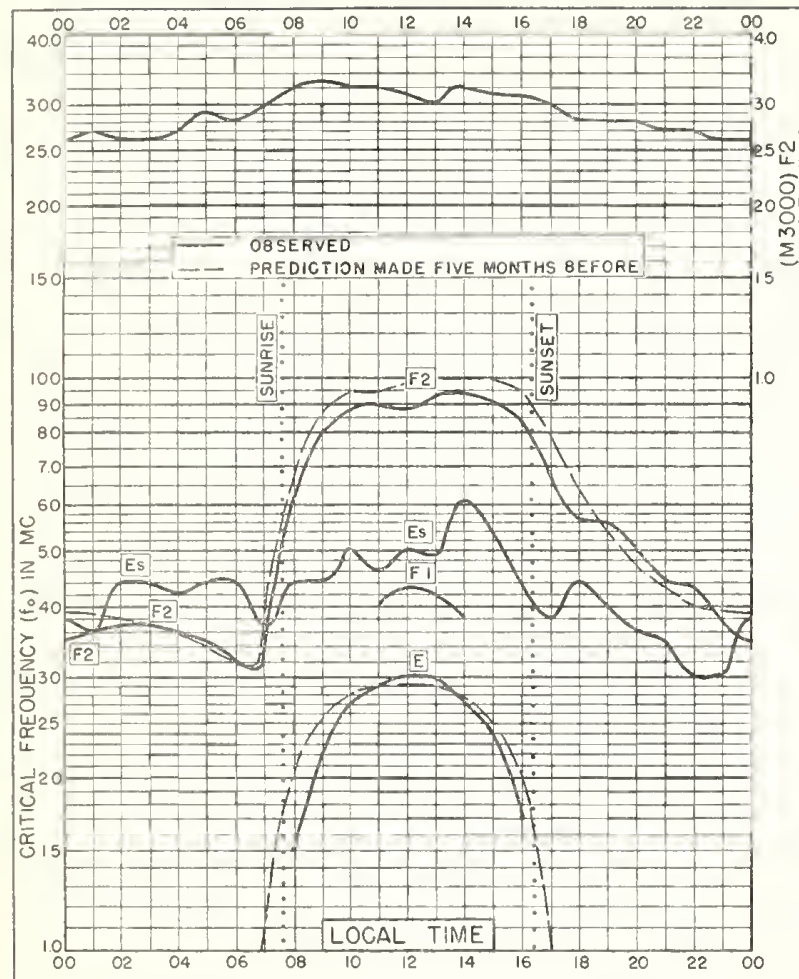

Fig 49 CHRISTCHURCH, N.Z $435^{\circ} \mathrm{S}, 172.7^{\circ} \mathrm{E}$

JUNE 1949

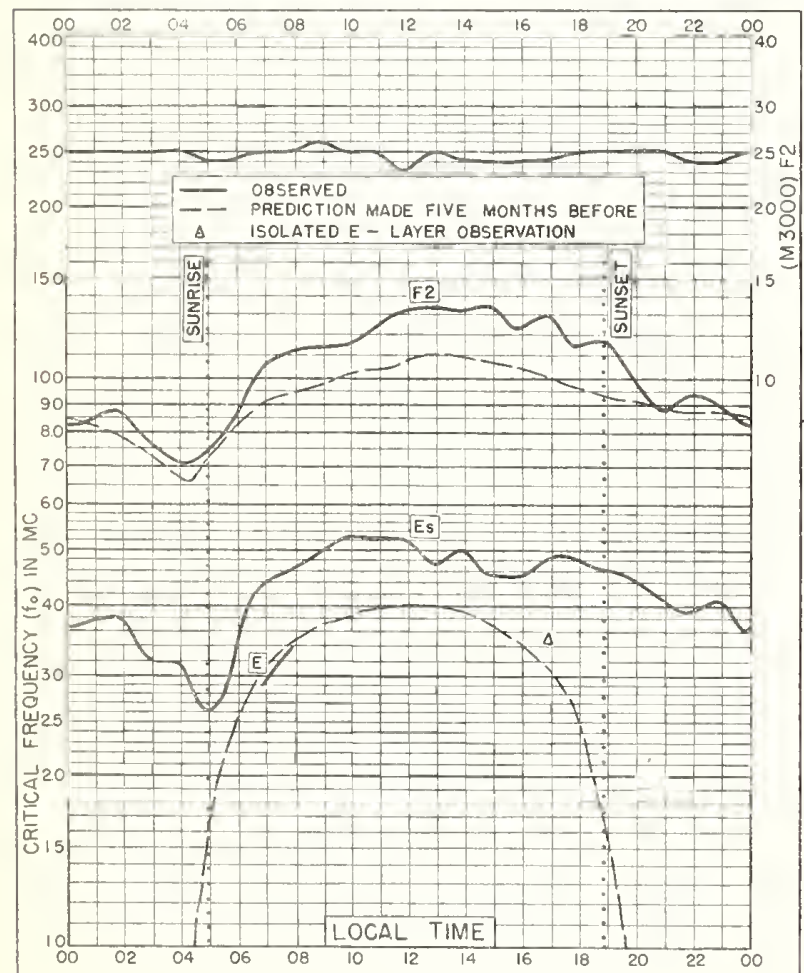

Fig. 51. LANCHOW, CHINA 36. $1^{\circ} \mathrm{N}, 103.8^{\circ} \mathrm{E}$
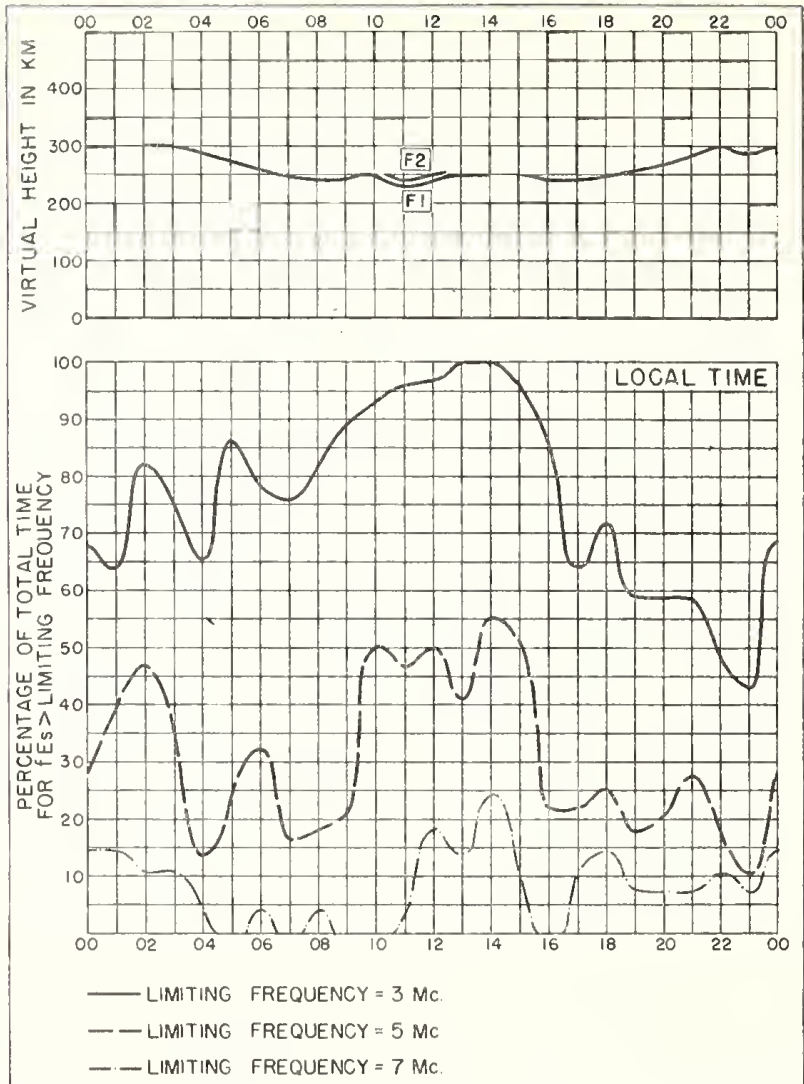

Fig. 50 CHRISTCHURCH, N.Z.

JUNE 1949
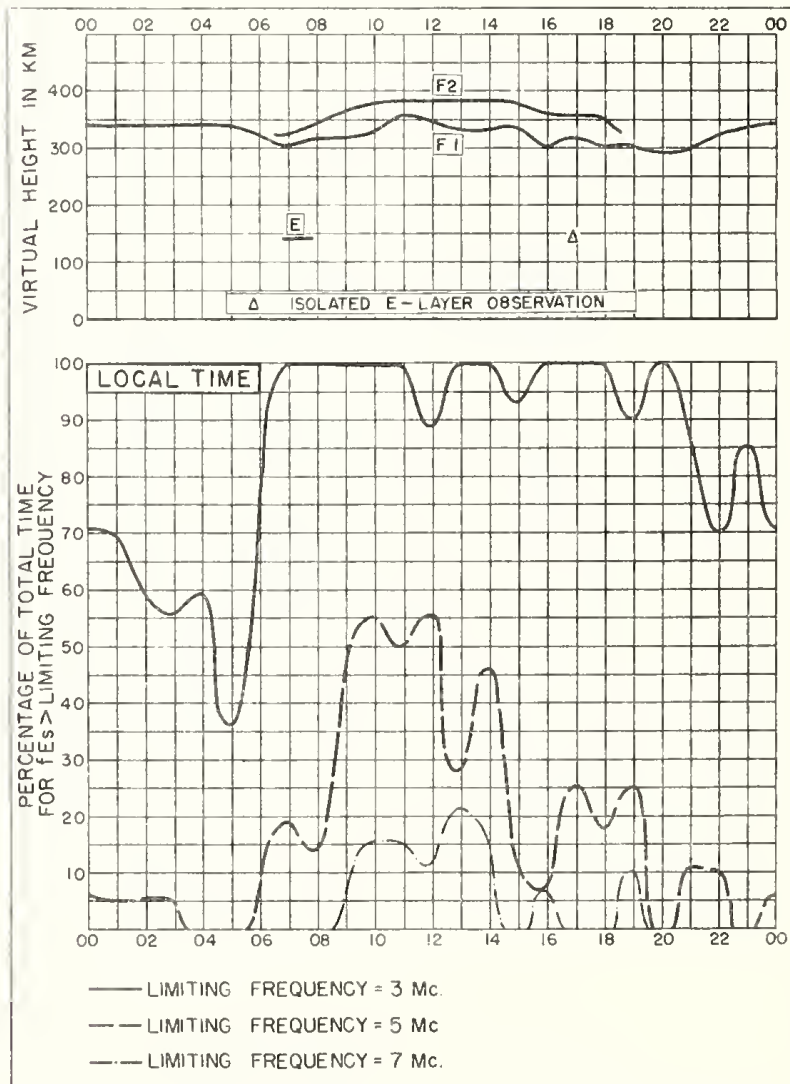

Fig. 52. LANCHOW, CHINA

MAY 1949 


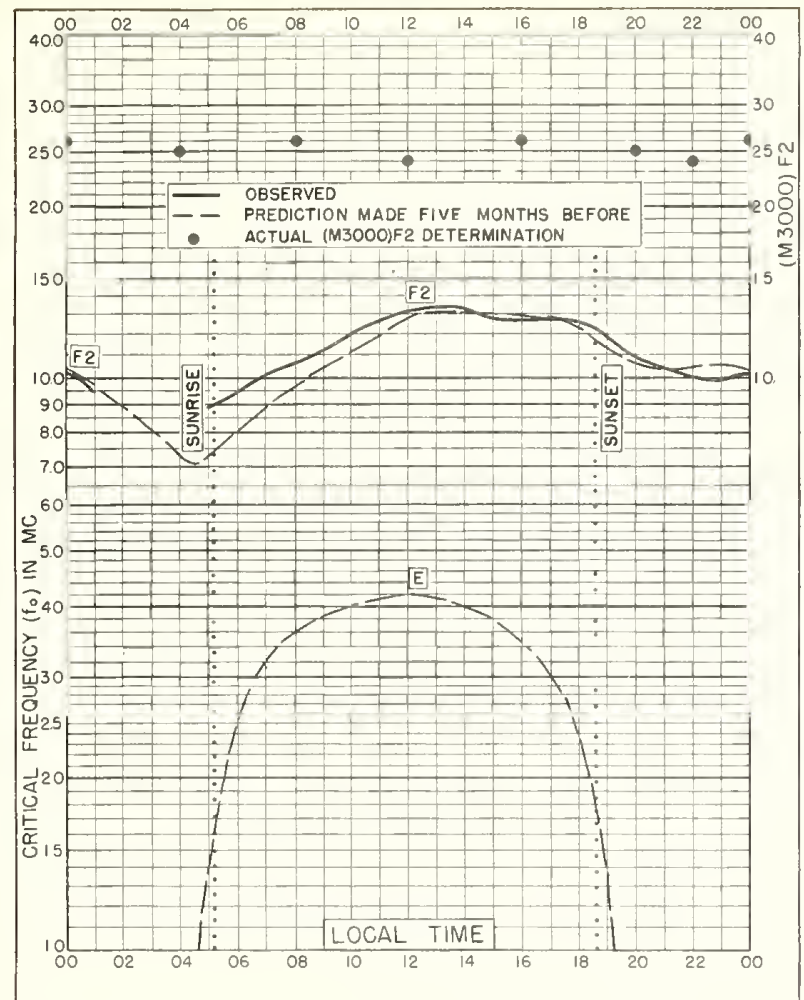

Fig. 53. DELHI, INDIA $28.6^{\circ} \mathrm{N}, 77.1^{\circ} \mathrm{E}$

MAY 1949

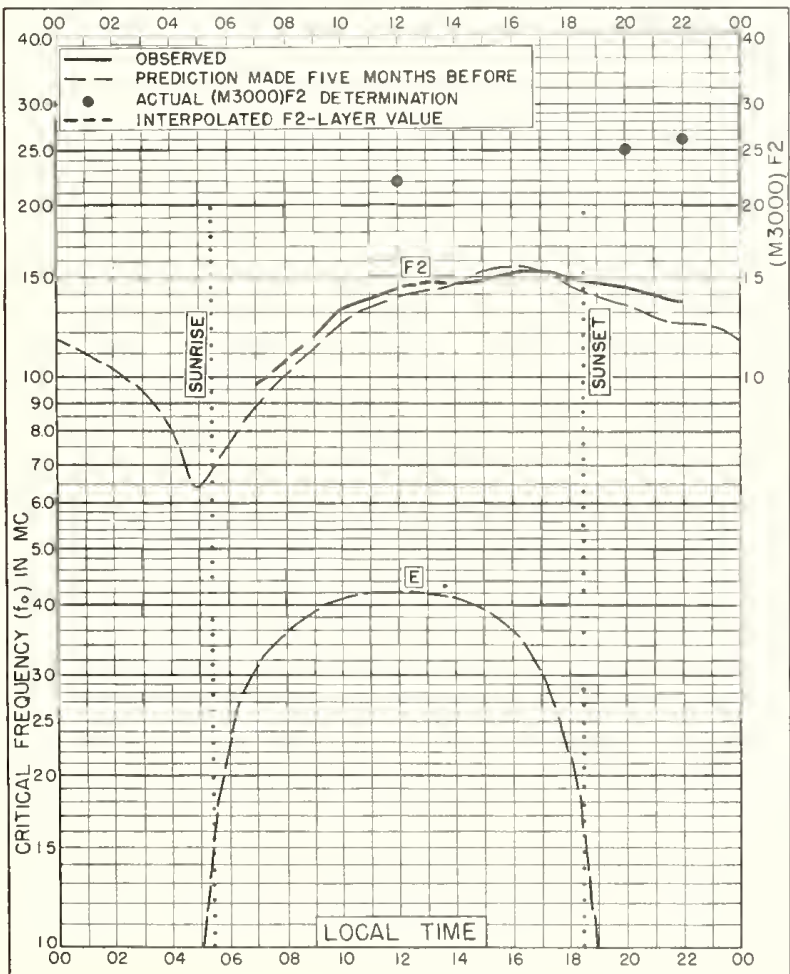

Fig. 55. BOMBAY, INDIA

19. $0^{\circ} \mathrm{N}, 73.0^{\circ} \mathrm{E}$
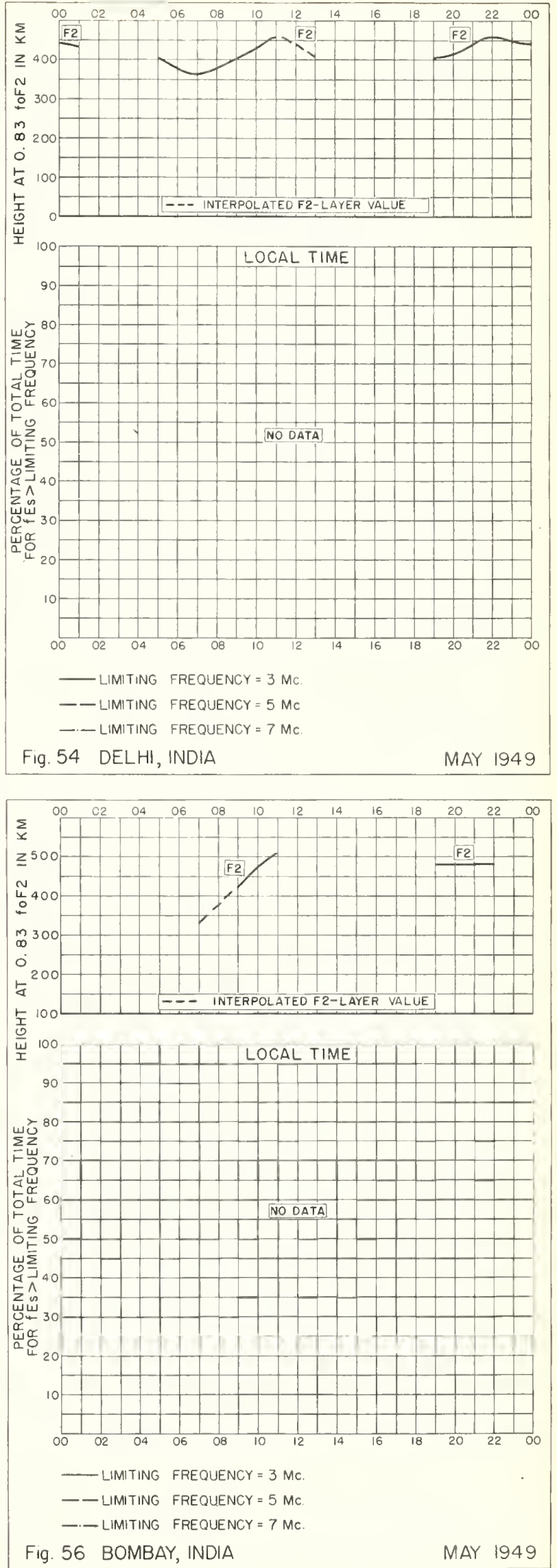


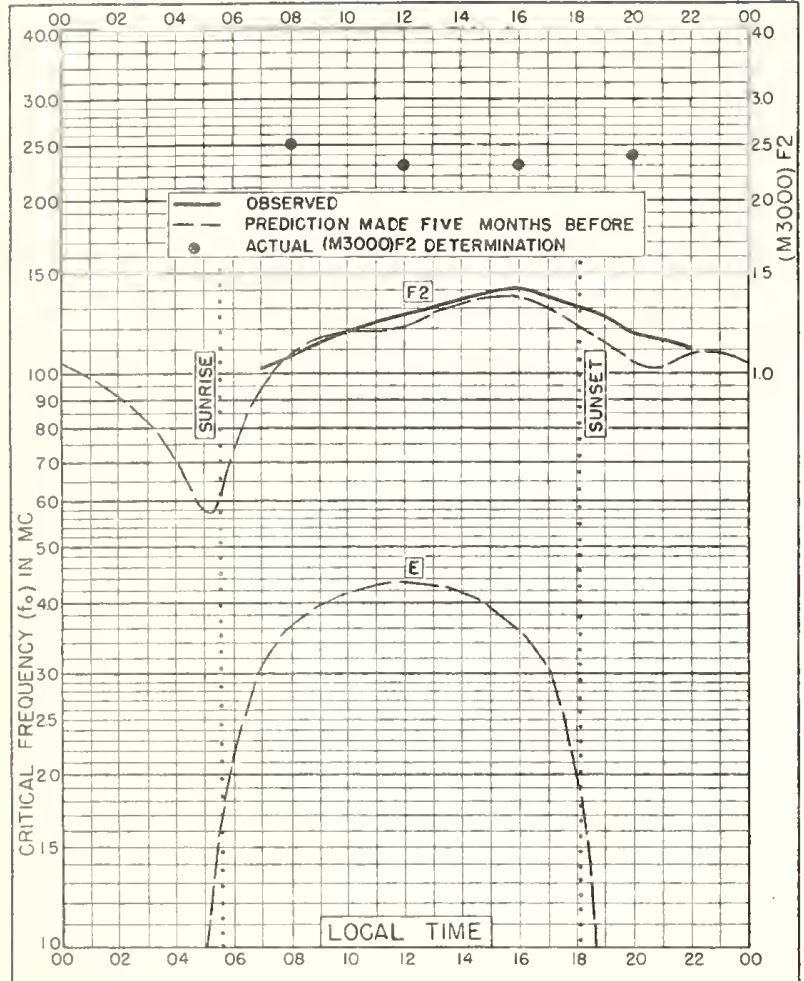

Fig. 57. MADRAS, INDIA $13.0^{\circ} \mathrm{N}, 80.2^{\circ} \mathrm{E}$

MAY 1949

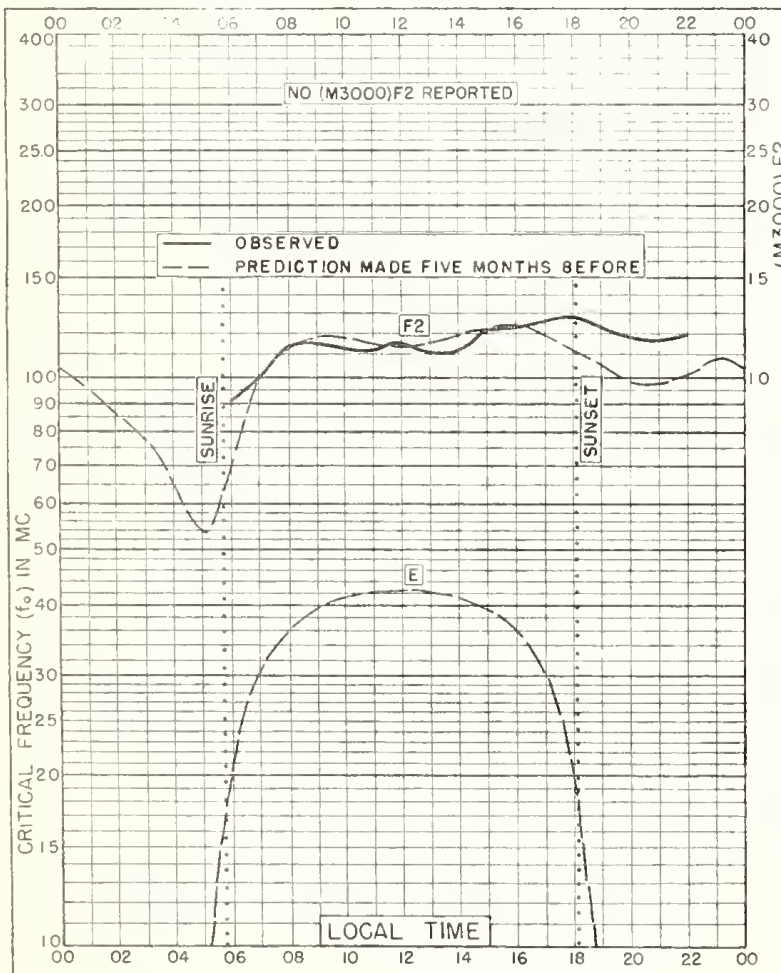

Fig. 59. TIRUCHIRAPALLI, INDIA $10.8^{\circ} \mathrm{N}, 78.8^{\circ} \mathrm{E}$

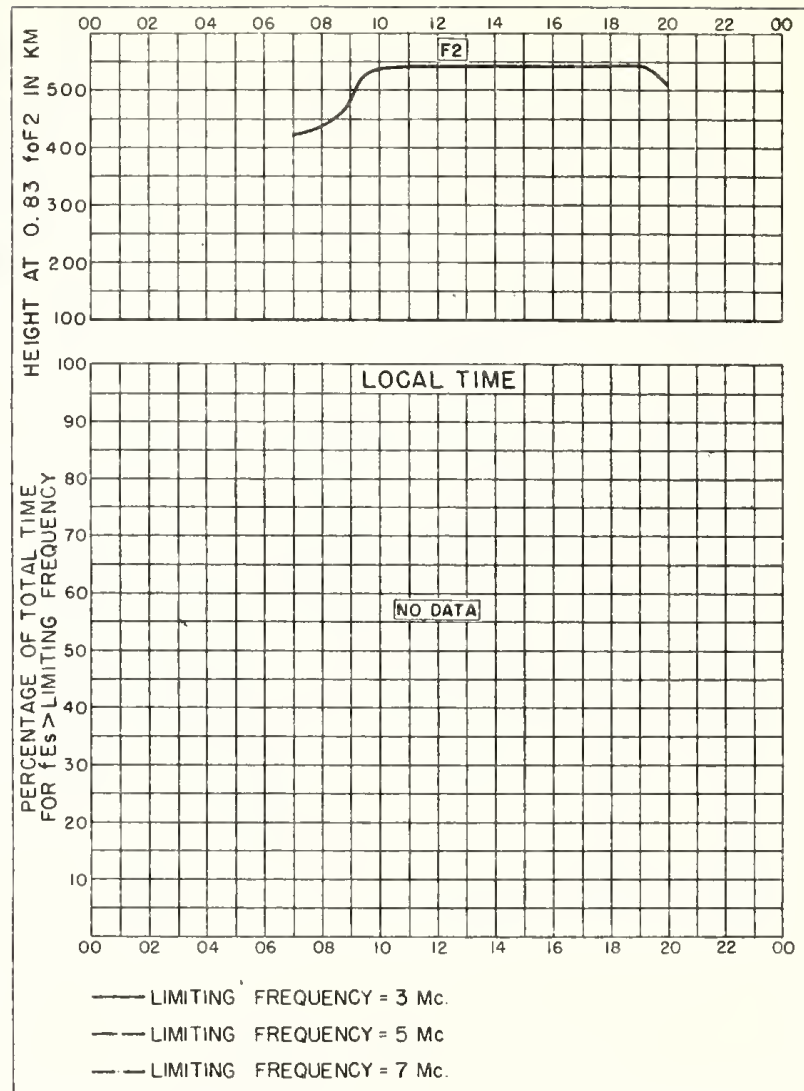

Fig. 58 MADRAS, INDIA

MAY 1949

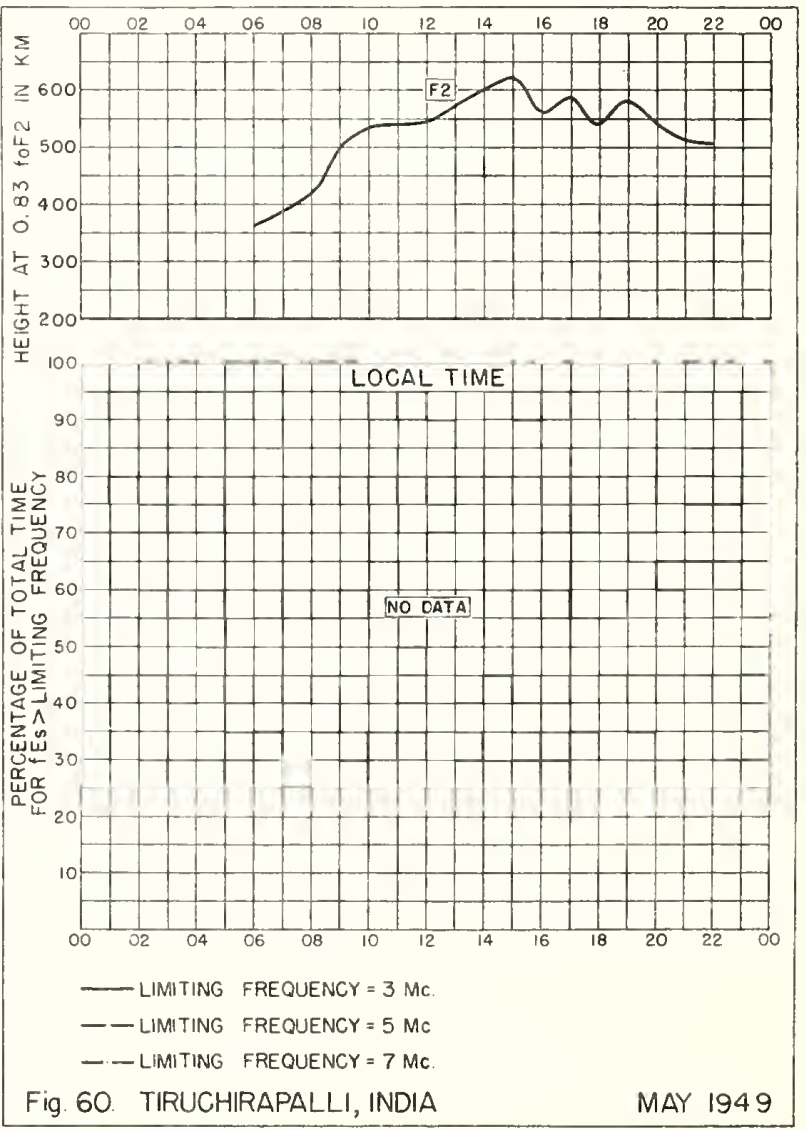




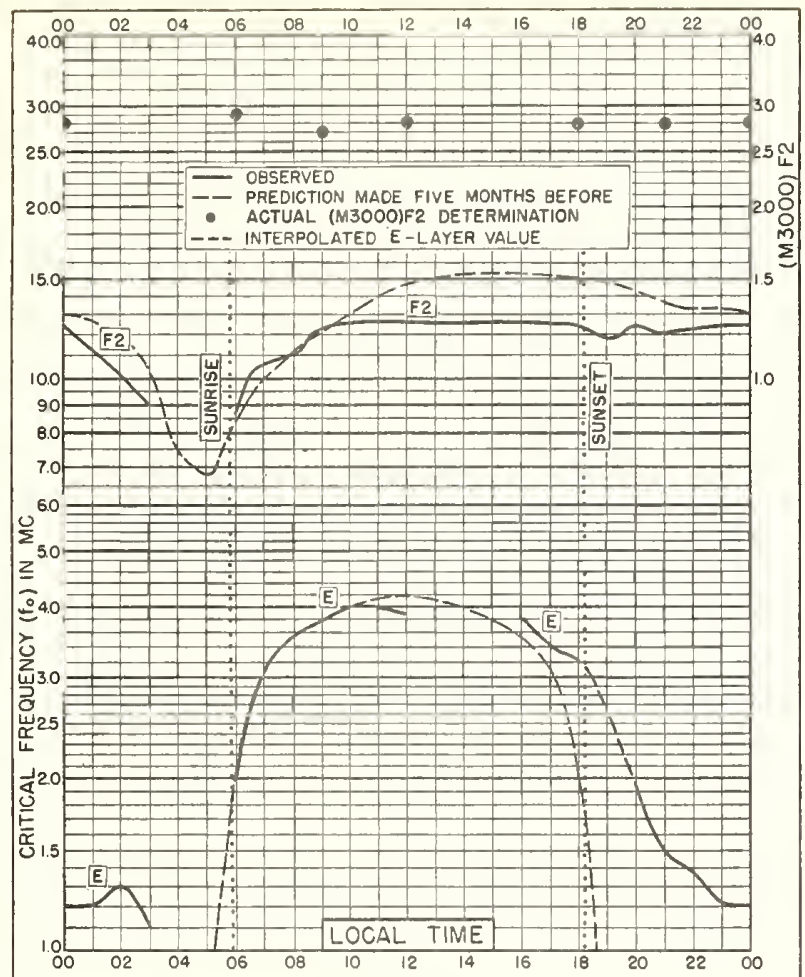

Fig. 61. CALCUTTA, INDIA $22.6^{\circ} \mathrm{N}, 88.4^{\circ} \mathrm{E}$ APRIL 1949

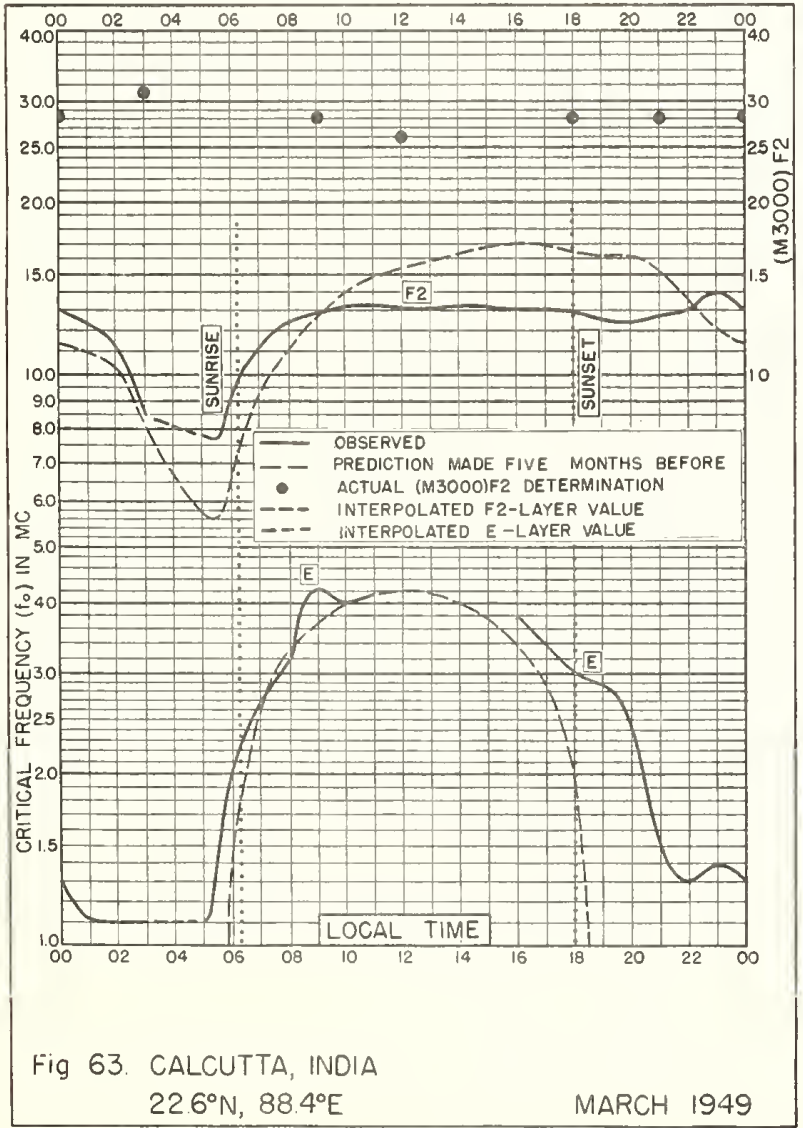

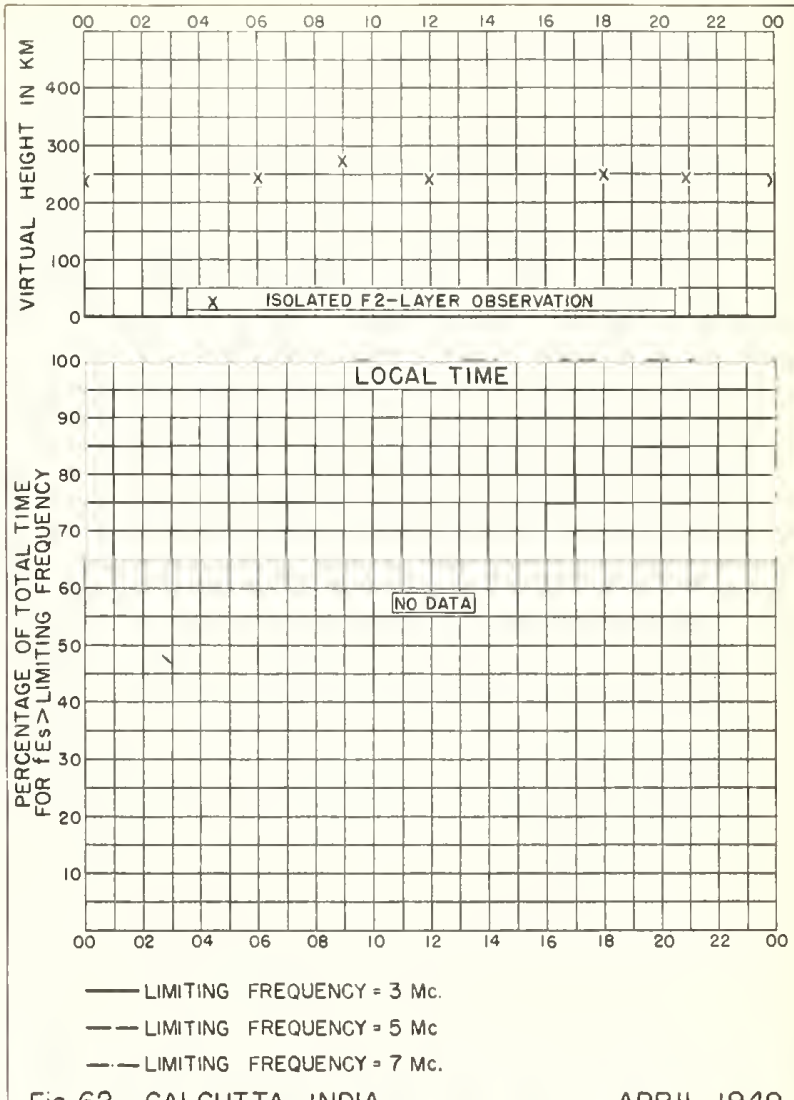

Fig. 62. CALCUTTA, INOIA

APRIL 1949
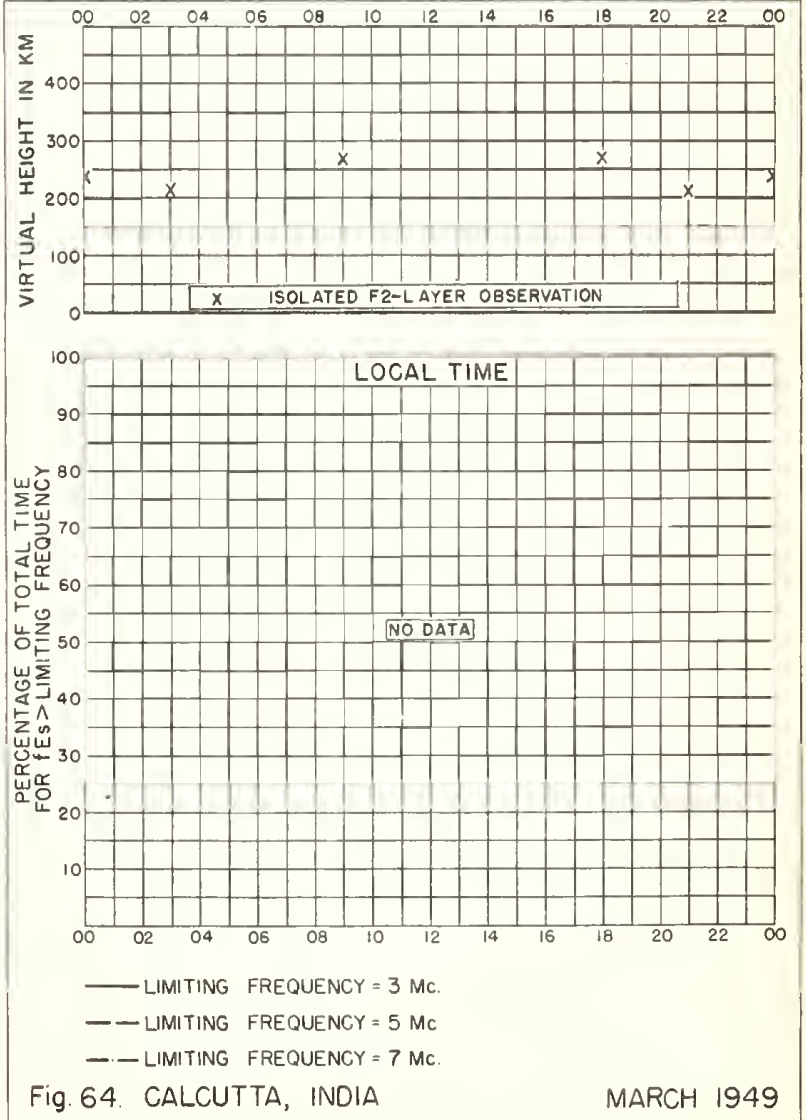


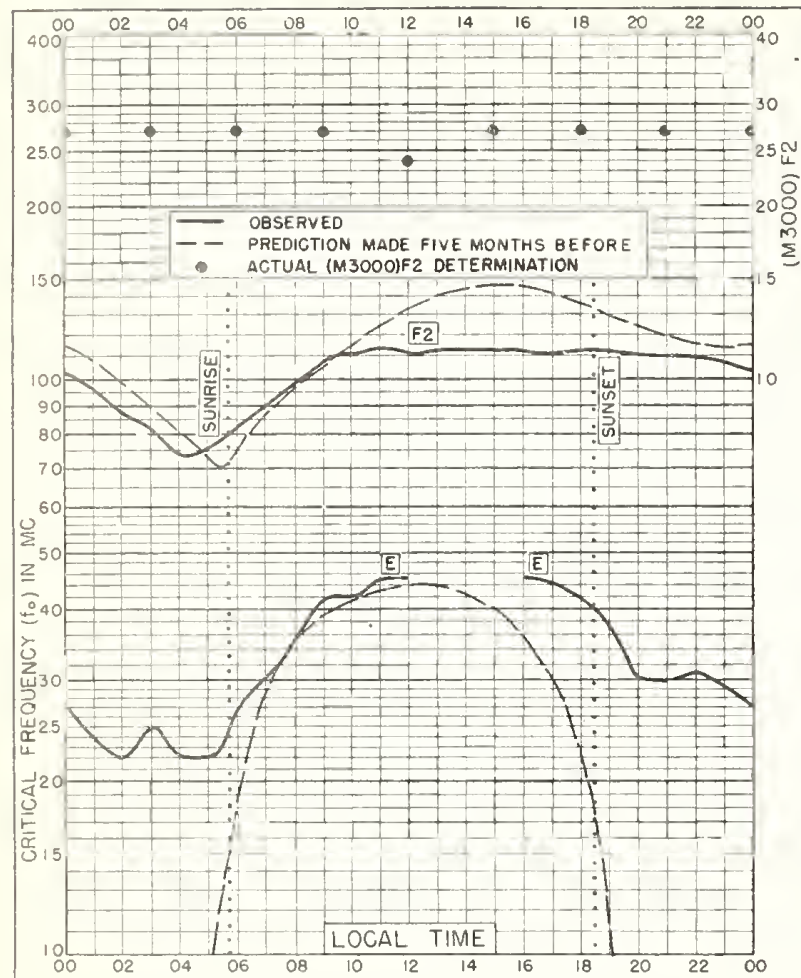

Fig. 65. CALCUTTA, INDIA $22.6^{\circ} \mathrm{N}, 88.4^{\circ} \mathrm{E}$

AUGUST 1948

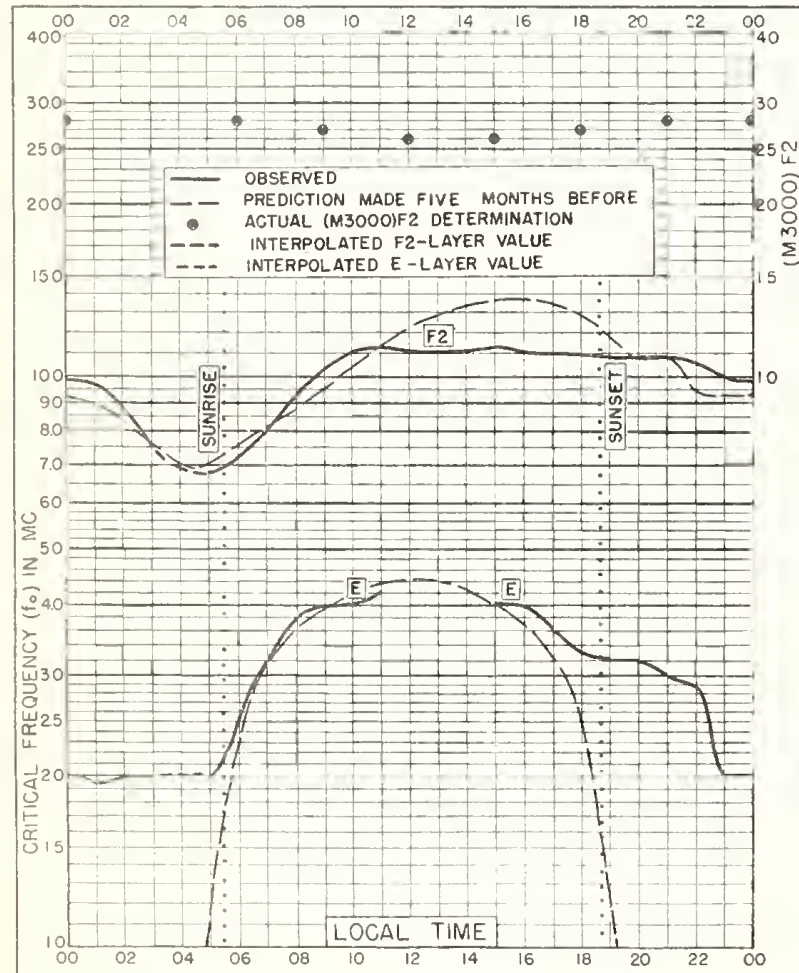

Fig. 67. CALGUTTA, INDIA $22.6^{\circ} \mathrm{N}, 88.4^{\circ} \mathrm{E}$ JULY 1948
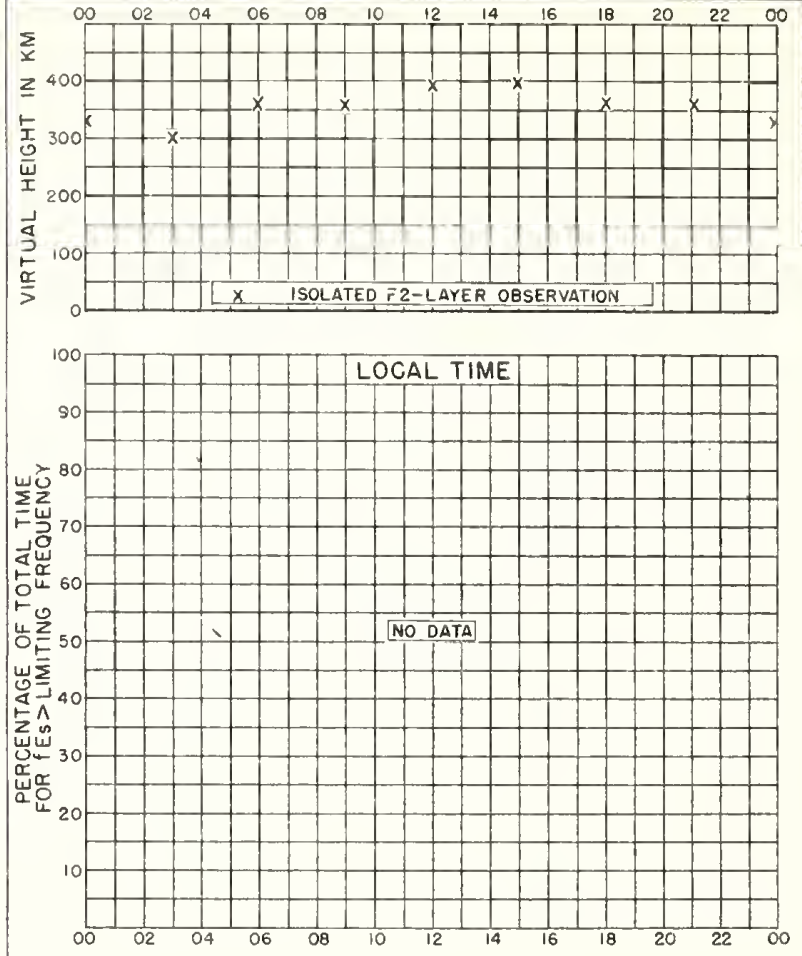

- LIMITING FREQUENCY $=3 \mathrm{Mc}$.
-- LIMITING FREQUENCY $=5 \mathrm{MC}$
-- LIMITING FREQUENCY $=7 \mathrm{Mc}$.

Fig. 66 CALCUTTA, INDIA

AUGUST 1948

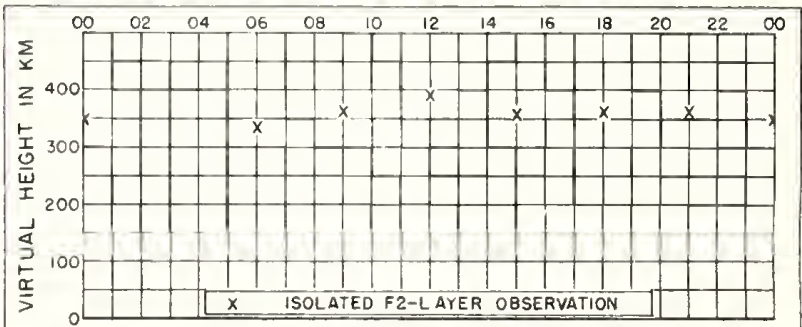

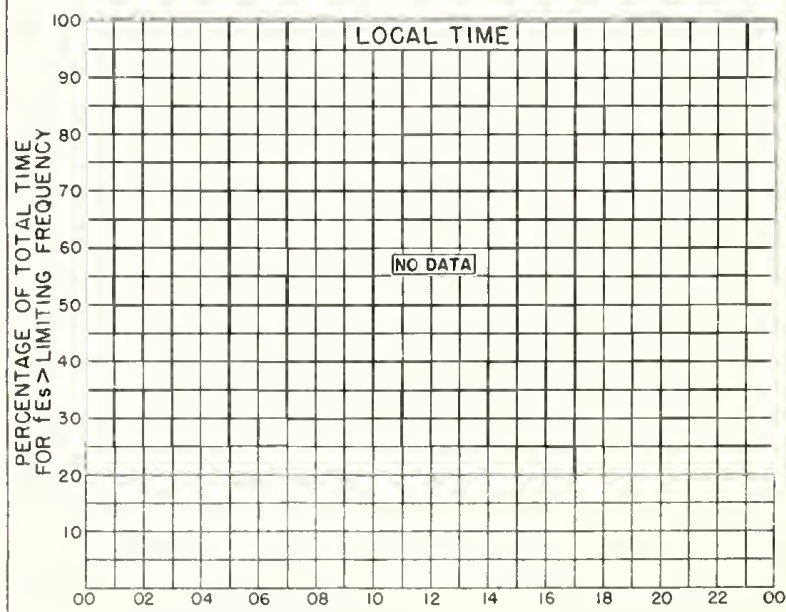

- LIMITING FREQUENCY $=3 \mathrm{MC}$

- - LIMITING FREQUENCY $=5 \mathrm{MC}$

- - LIMITING FREQUENCY $=7 \mathrm{MC}$.

Fig. 68 CALCUTTA, INDIA

JULY 1948 


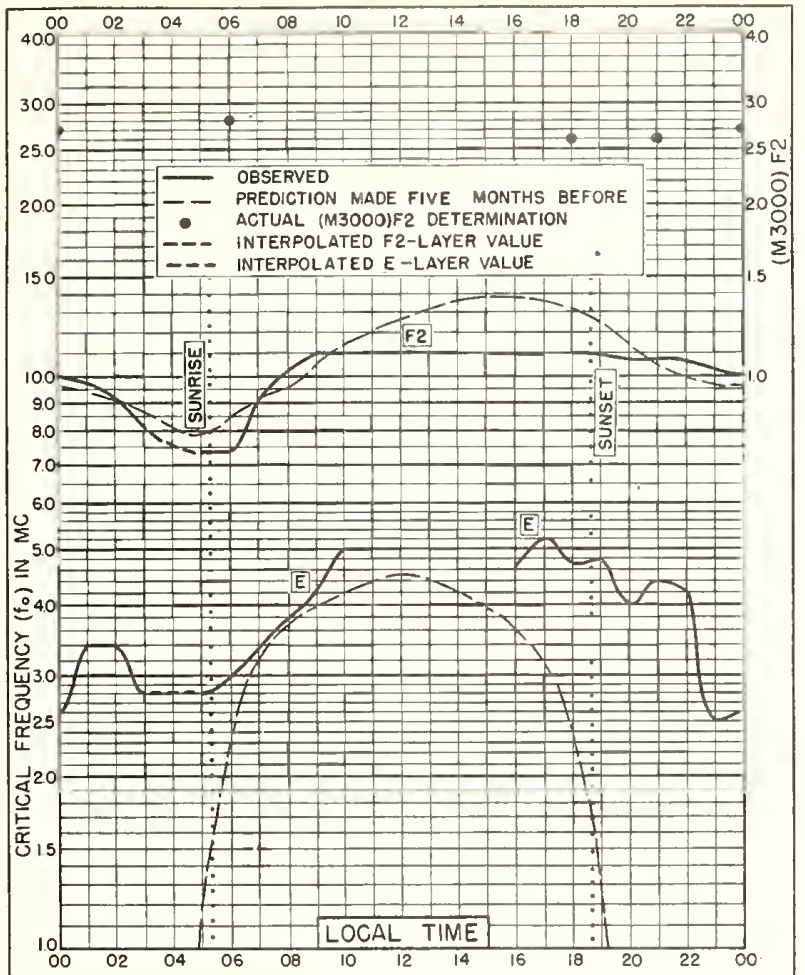

Fig 69. CALCUTTA, INDIA $22.6^{\circ} \mathrm{N}, 884^{\circ} \mathrm{E}$ JUNE 1948

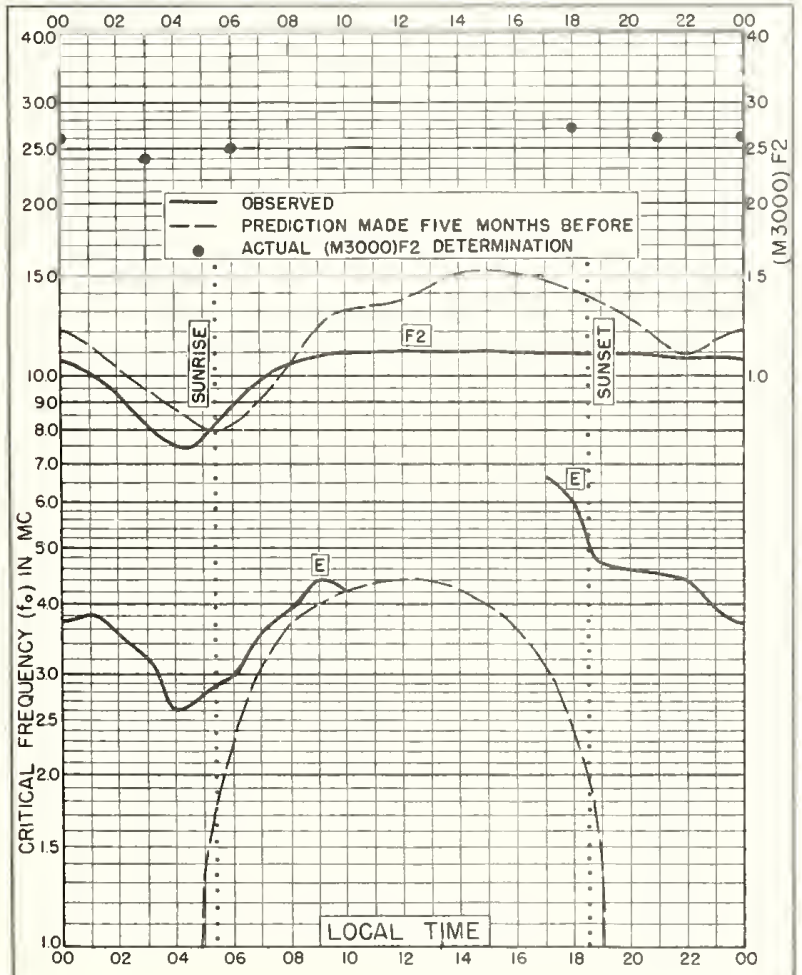

Fig. 7I. CALCUTTA, INDIA $22.6^{\circ} \mathrm{N}, 88.4^{\circ} \mathrm{E}$

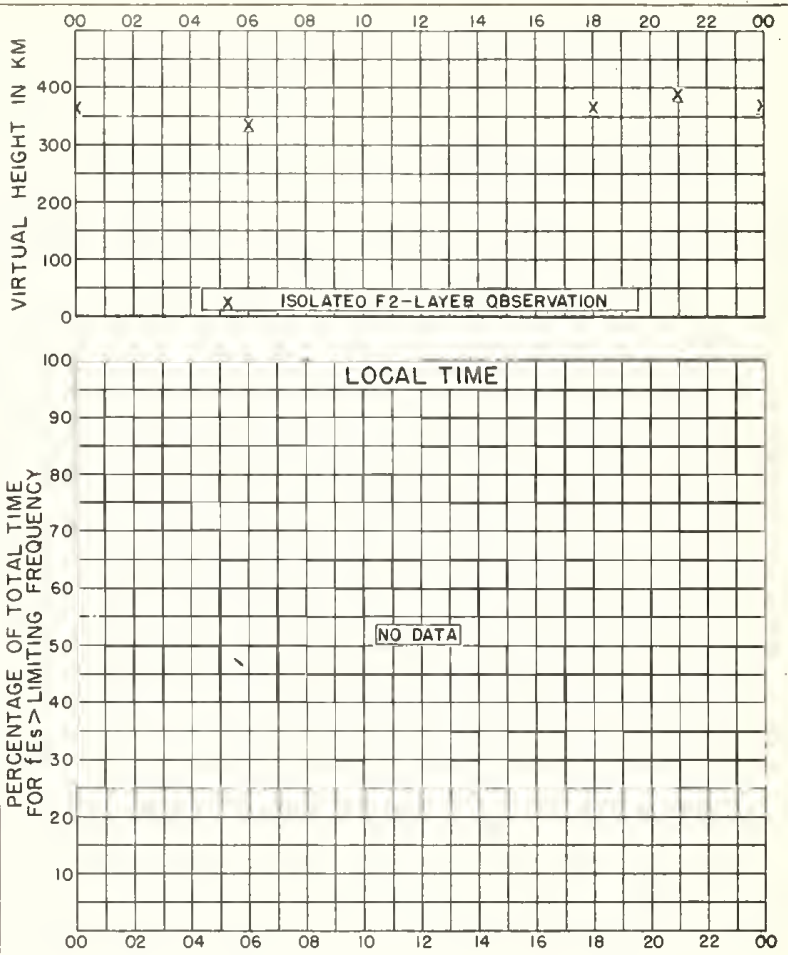

LIMITING FREQUENCY $=3 \mathrm{MC}$

- LIMITING FREQUENCY $=5 \mathrm{MC}$

- LIMITING FREQUENCY $=7 \mathrm{MC}$

Fig 70. CALCUTTA, INDIA

JUNE 1948
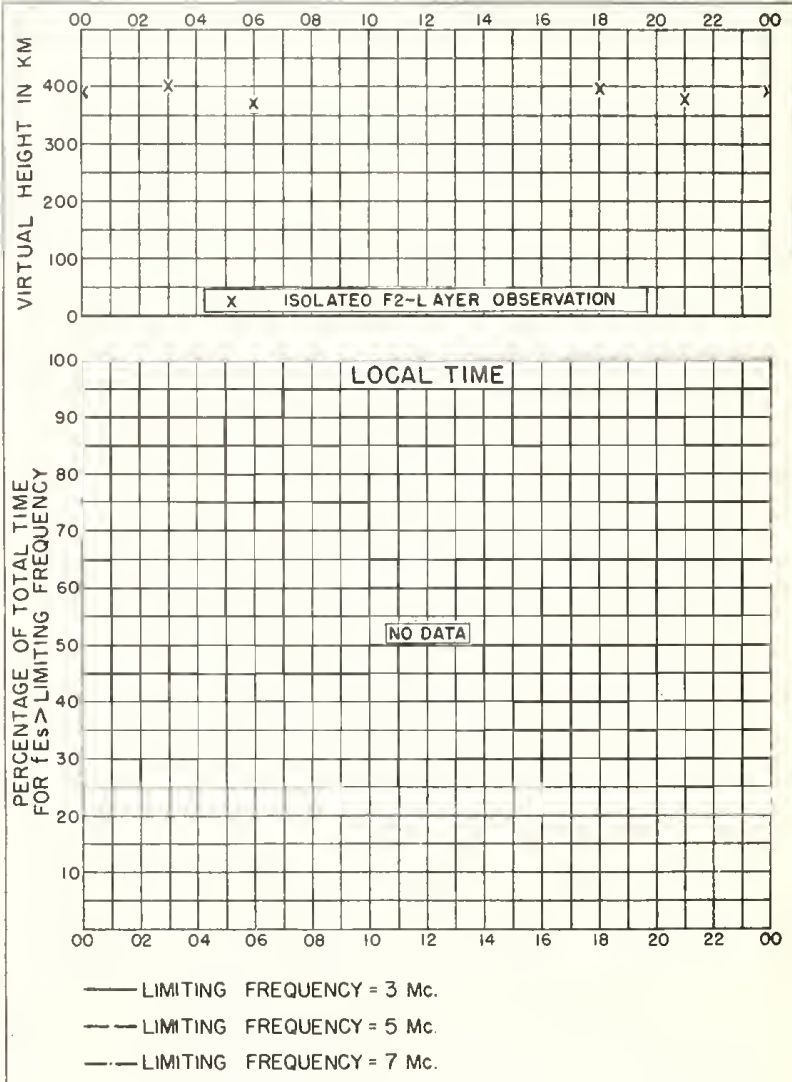

Fig. 72. CALCUTTA, INDIA

MAY 1948 


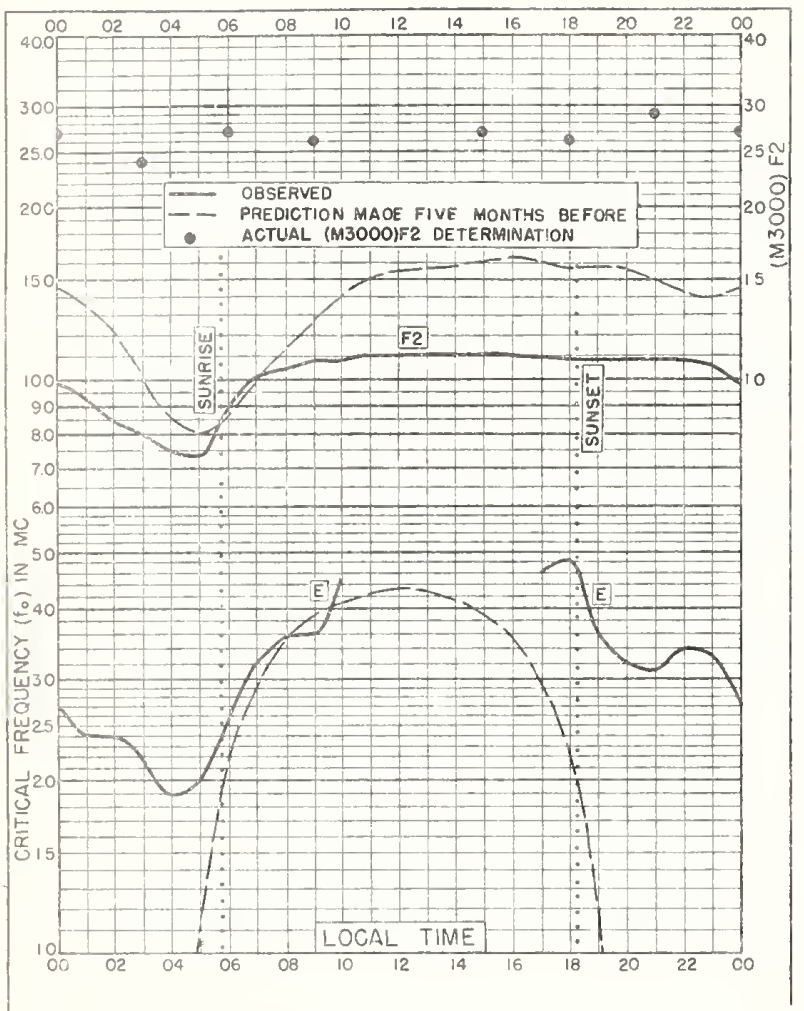

Fig. 73. CALCUTTA, INDIA

$22.6^{\circ} \mathrm{N}, 88.4^{\circ} \mathrm{E}$

APRIL 1948

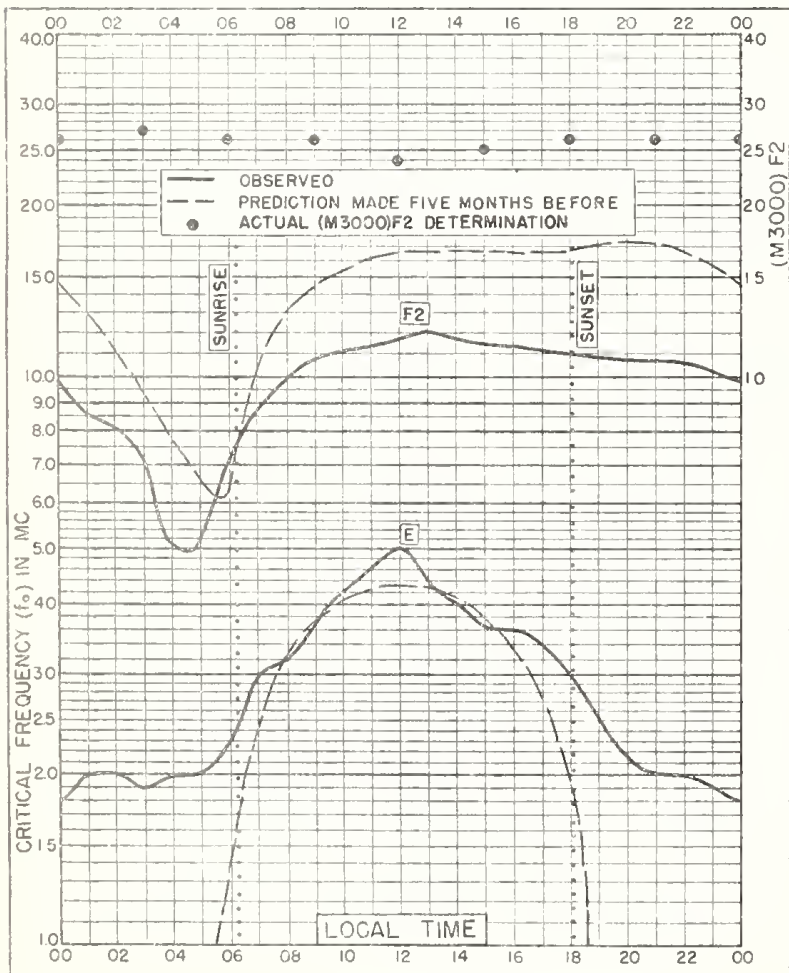

Fig. 75. CALCUTTA, INDIA

$226^{\circ} \mathrm{N}, 884^{\circ} \mathrm{E}$

MARCH 1948
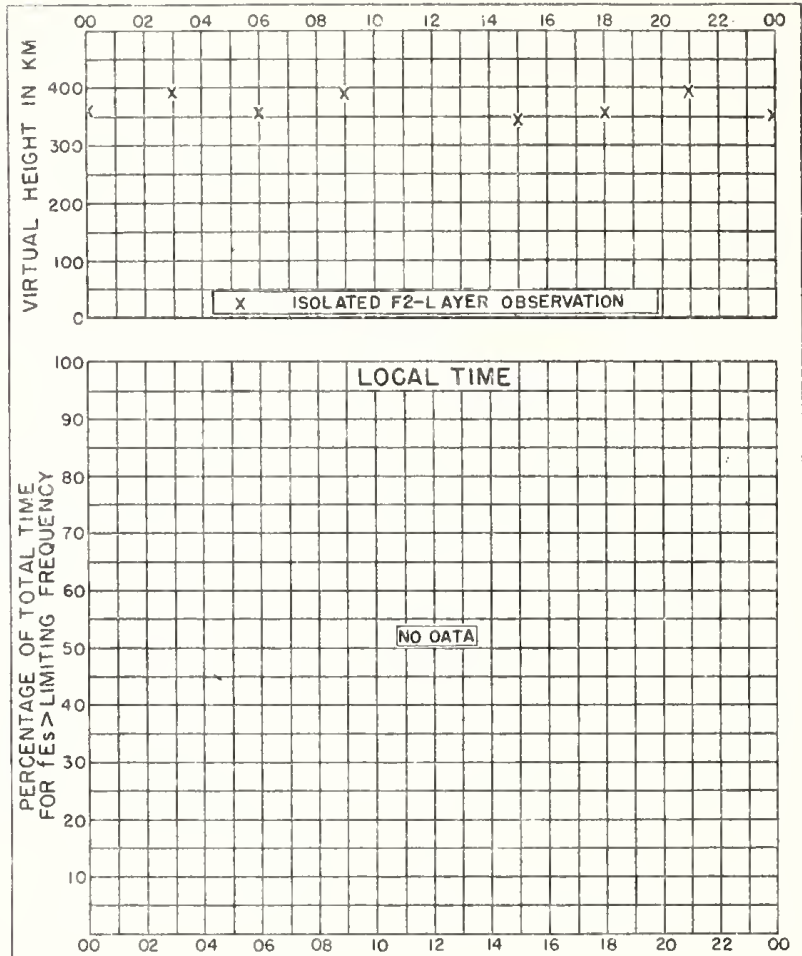

- LIMITING FREQUENCY $=3 \mathrm{MC}$

- LIMITING FREQUENCY $=5 \mathrm{MC}$

- - LIMITING FREQUENCY $=7 \mathrm{Mc}$

Fig 74. CALCUTTA, INDIA

APRIL 1948
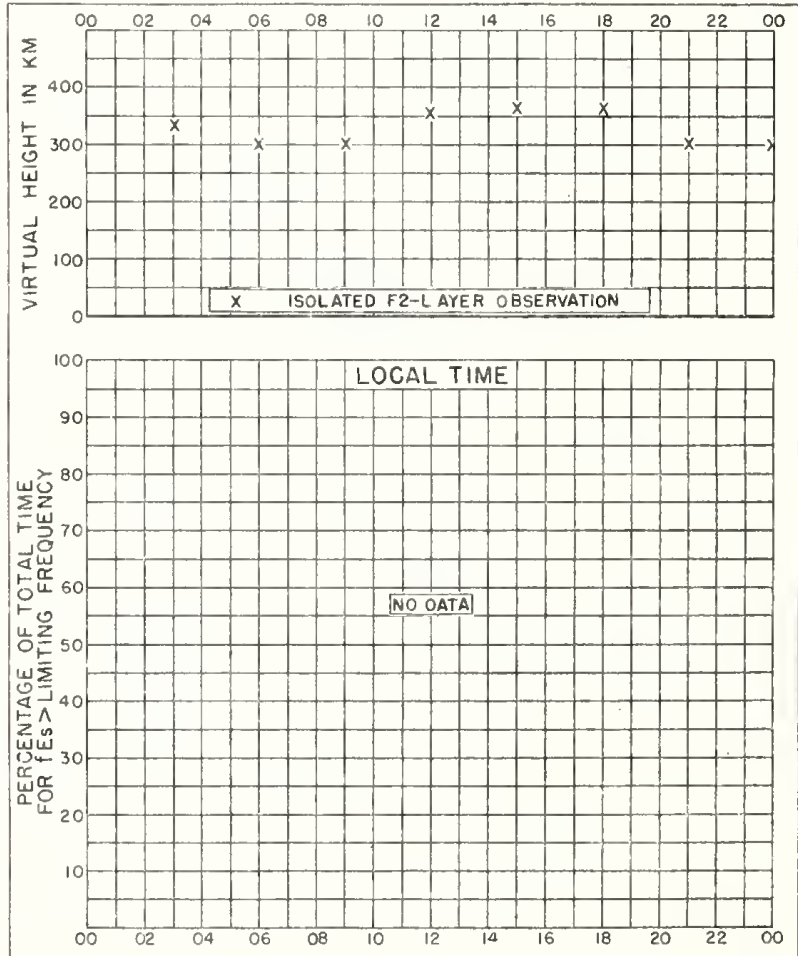

LIMITING FREQUENCY $=3 \mathrm{MC}$

- LIMITING FREQUENCY $=5 \mathrm{MC}$

- - LIMITING FREQUENCY $=7 \mathrm{MC}$

Fig. 76. CALCUTTA; INDIA

MARCH 1948 


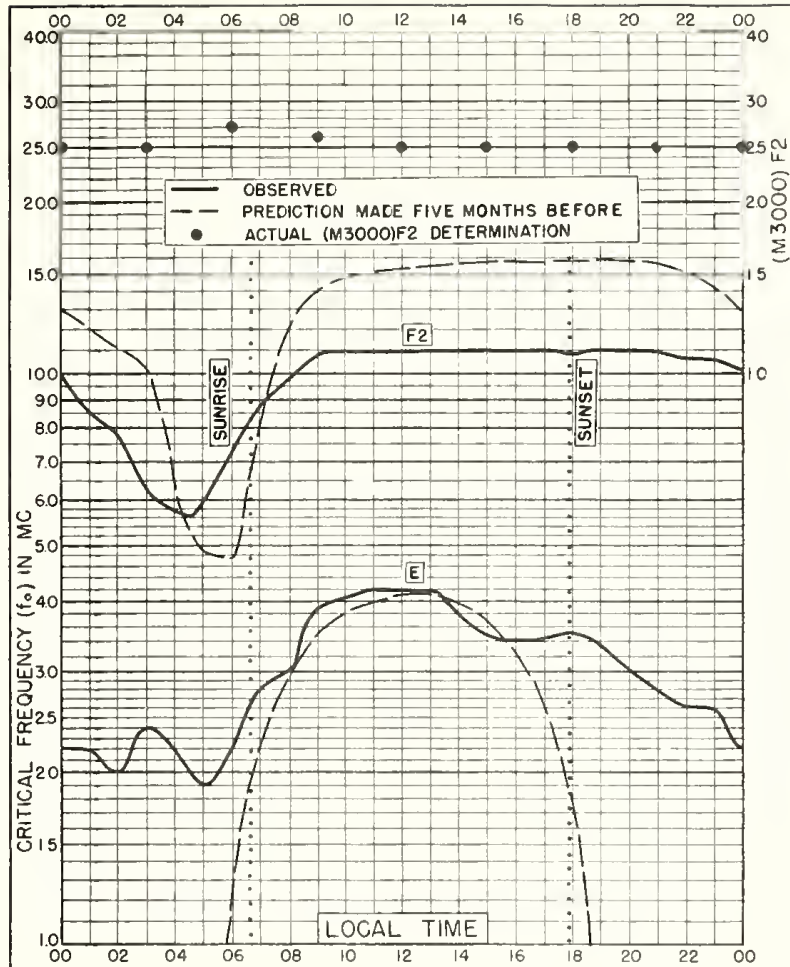

Fig 77 CALCUTTA, INDIA $22.6^{\circ} \mathrm{N}, 88.4^{\circ} \mathrm{E}$

FEBRUARY 1948

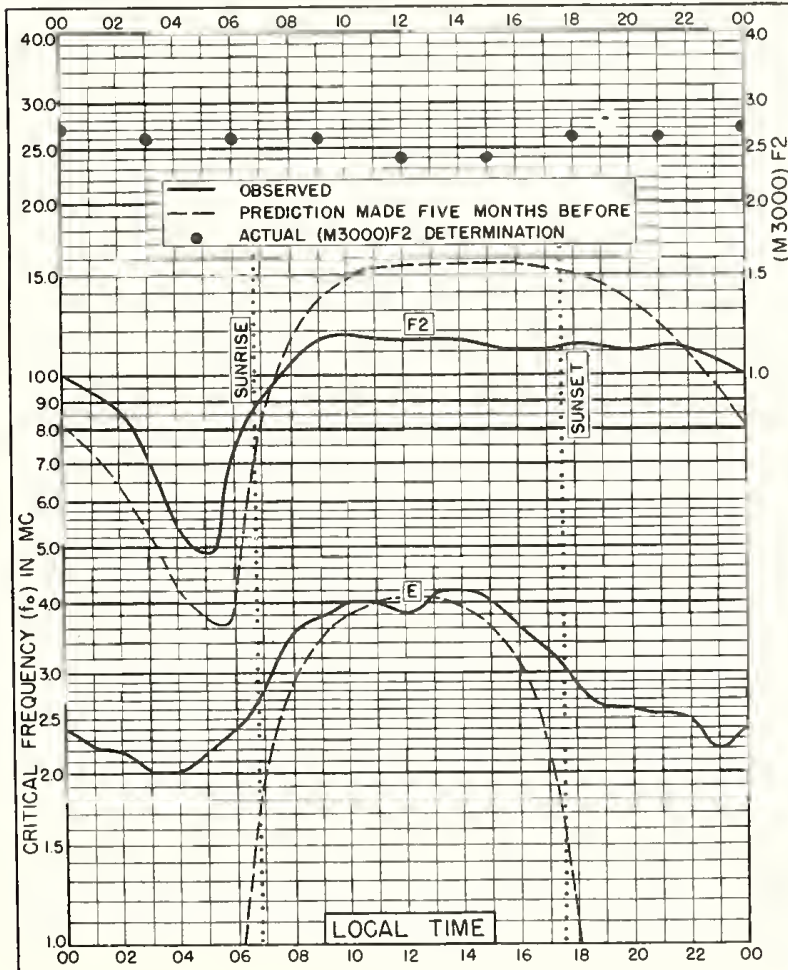

Fig. 79. CALCUTTA, INDIA $22.6^{\circ} \mathrm{N}, 88.4^{\circ} \mathrm{E}$
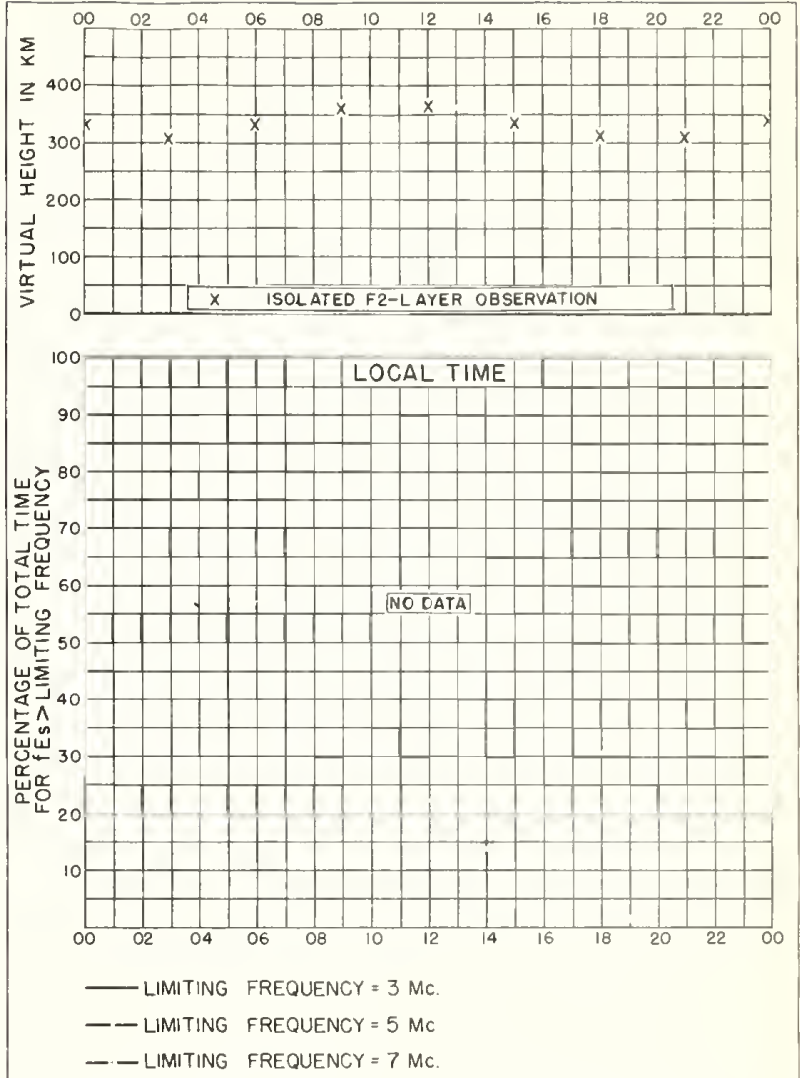

Fig 78. CALCUTTA, INOIA FEBRUARY 1948
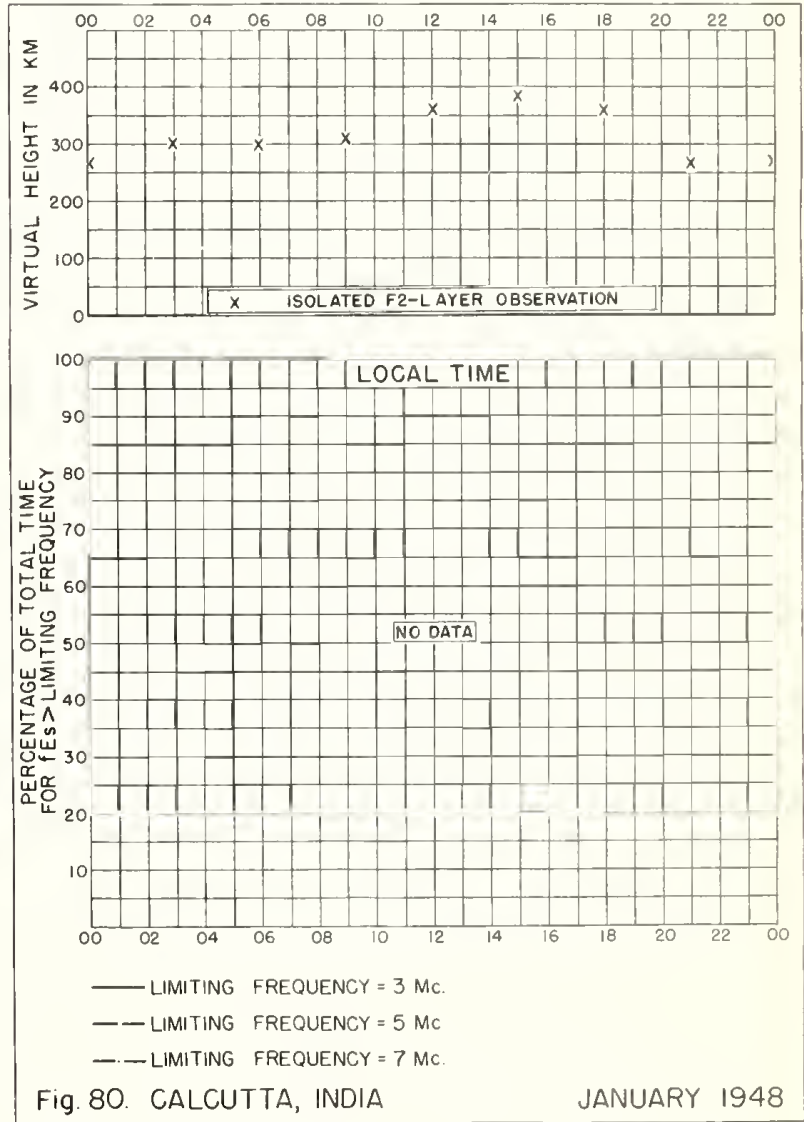
62

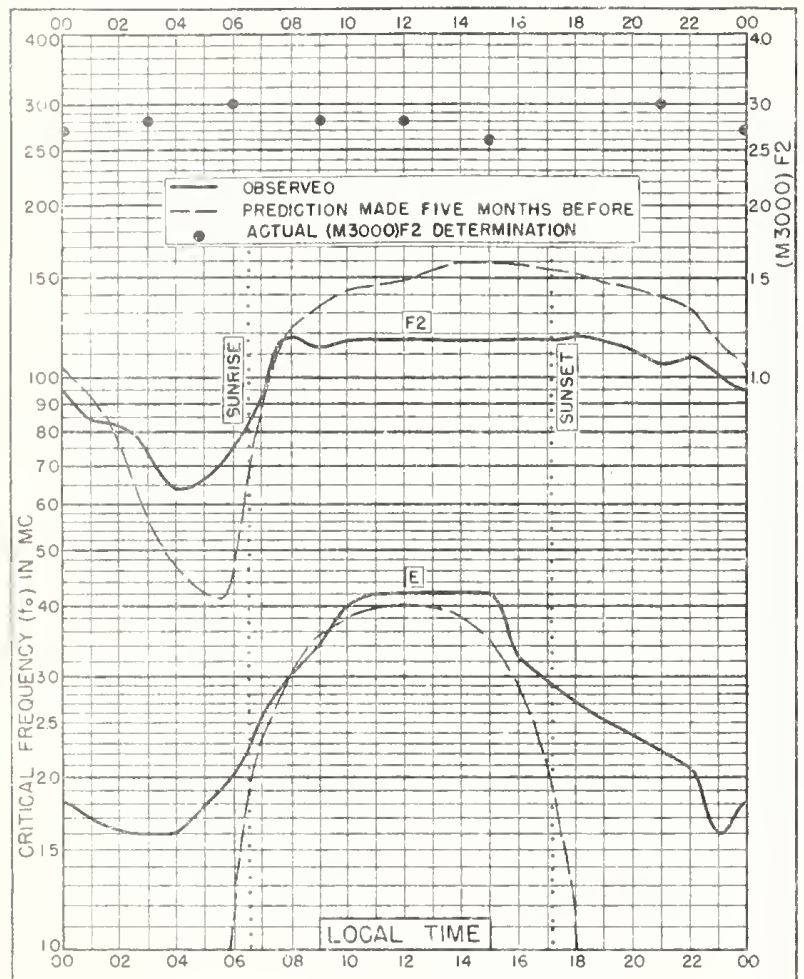

Fig 81 CALCUTTA, INDIA $22.6^{\circ} \mathrm{N}, 884^{\circ} \mathrm{E}$ DECEMBER 1947

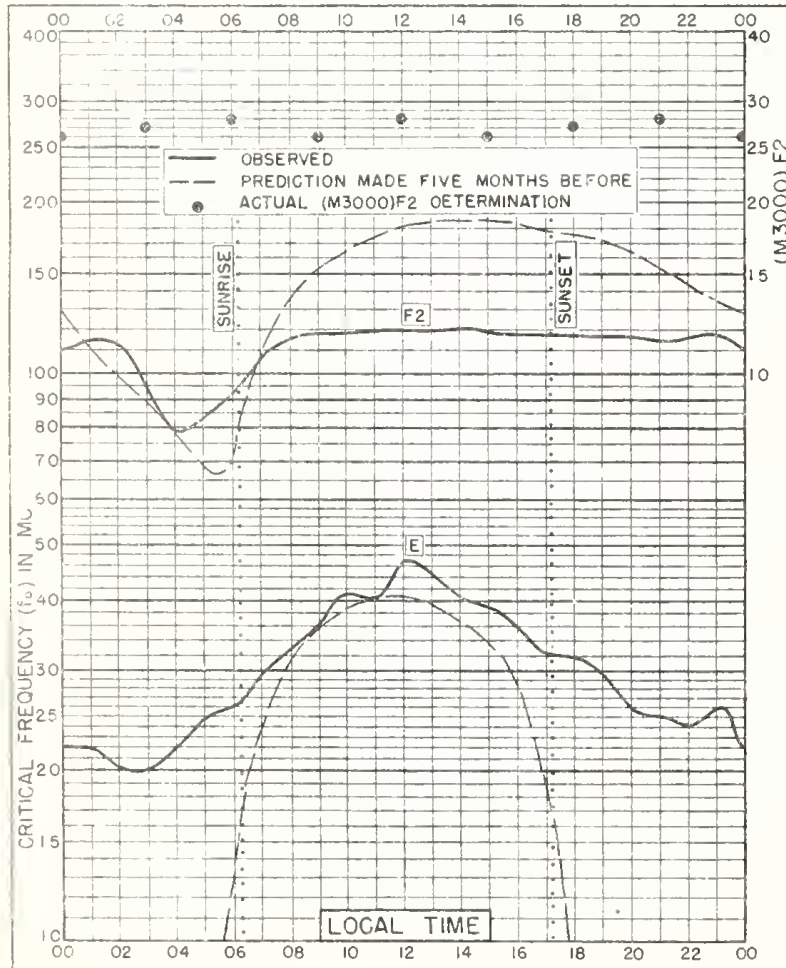

FIg. 83. CALCUTTA, INDIA $226^{\circ} \mathrm{N}, 884^{\circ} \mathrm{E}$
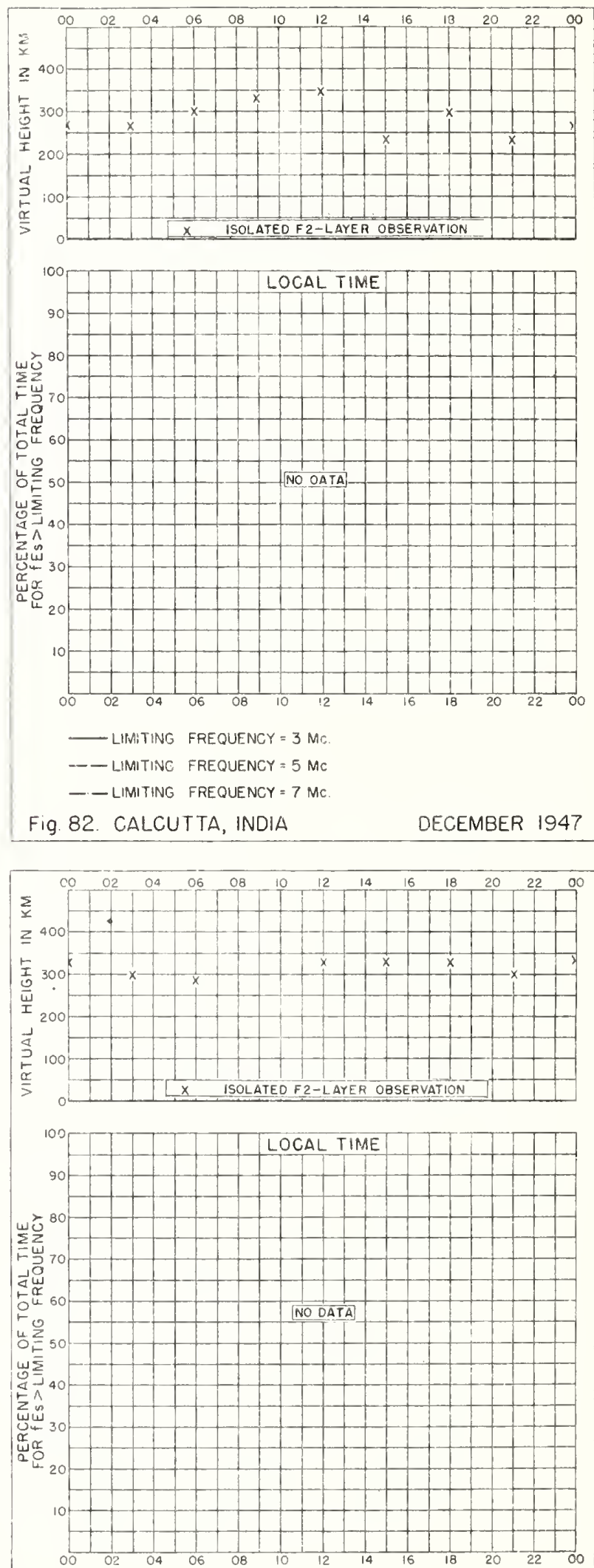

\footnotetext{
LIMITING FREQUENCY $=3 \mathrm{MC}$

- LIMITING FREQUENCY $=5 \mathrm{MC}$

- - LIMITING FREQUENCY $=7 \mathrm{MC}$
}

Fig 84. CALCUTTA, INDIA 


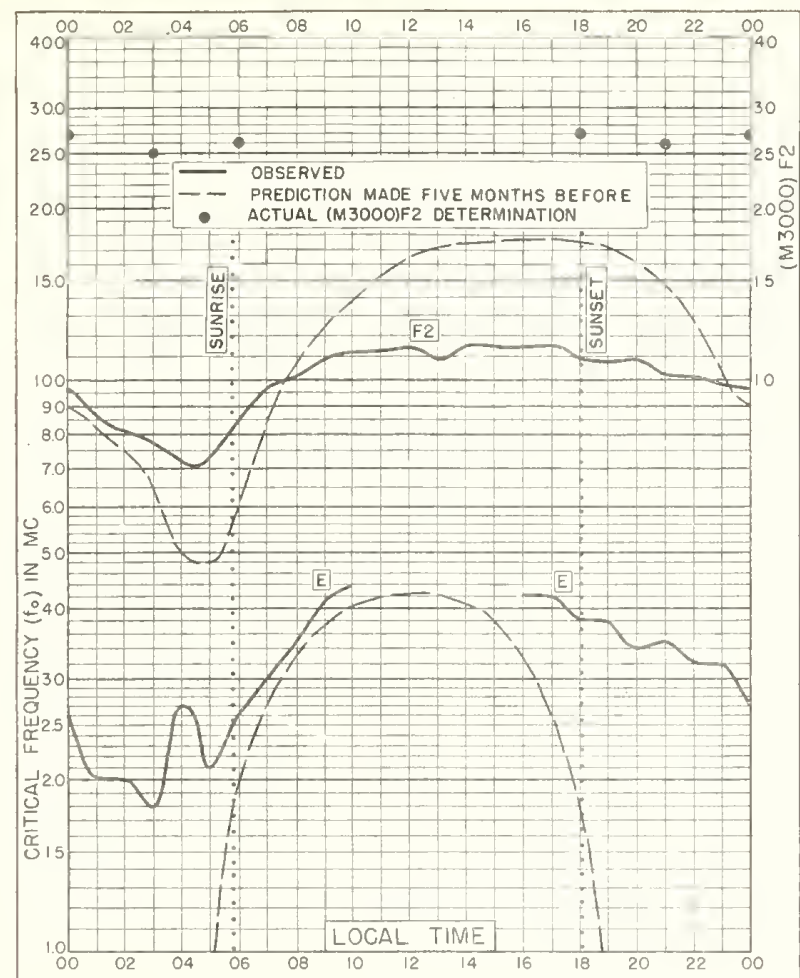

Fig. 85. CALCUTTA, INOIA $22.6^{\circ} \mathrm{N}, 88.4^{\circ} \mathrm{E}$
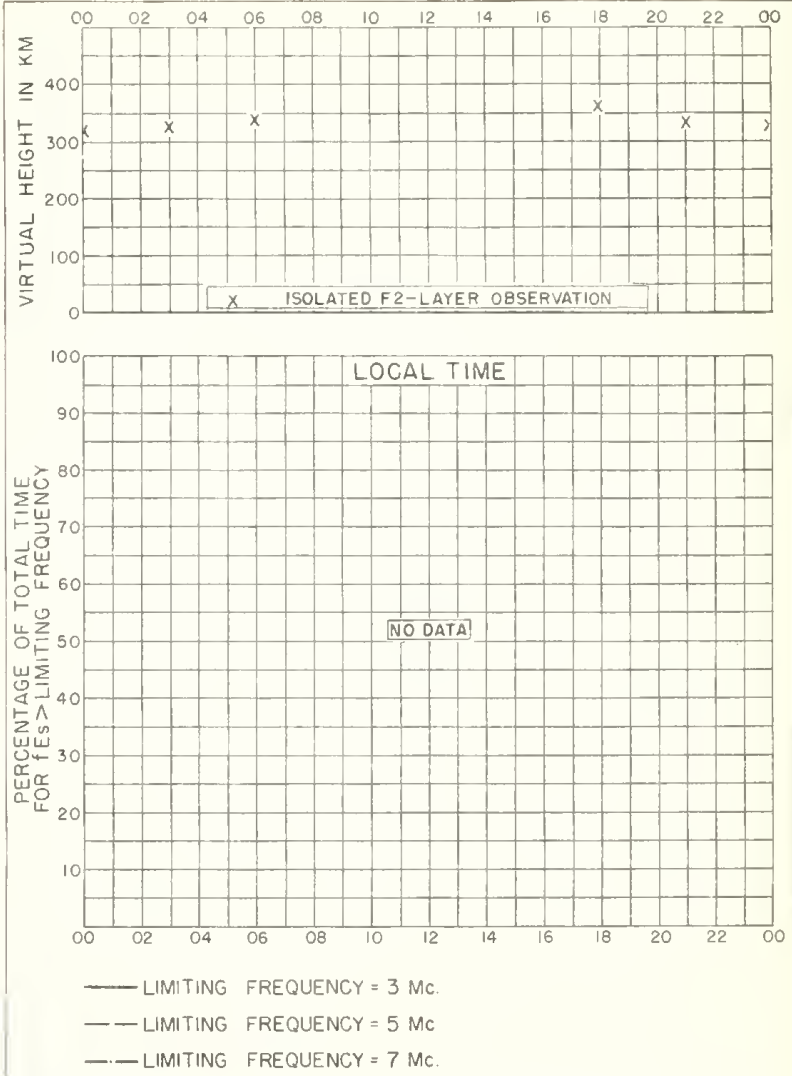

Fig. 86. CALCUTTA, INDIA

SEPTEMBER 1947
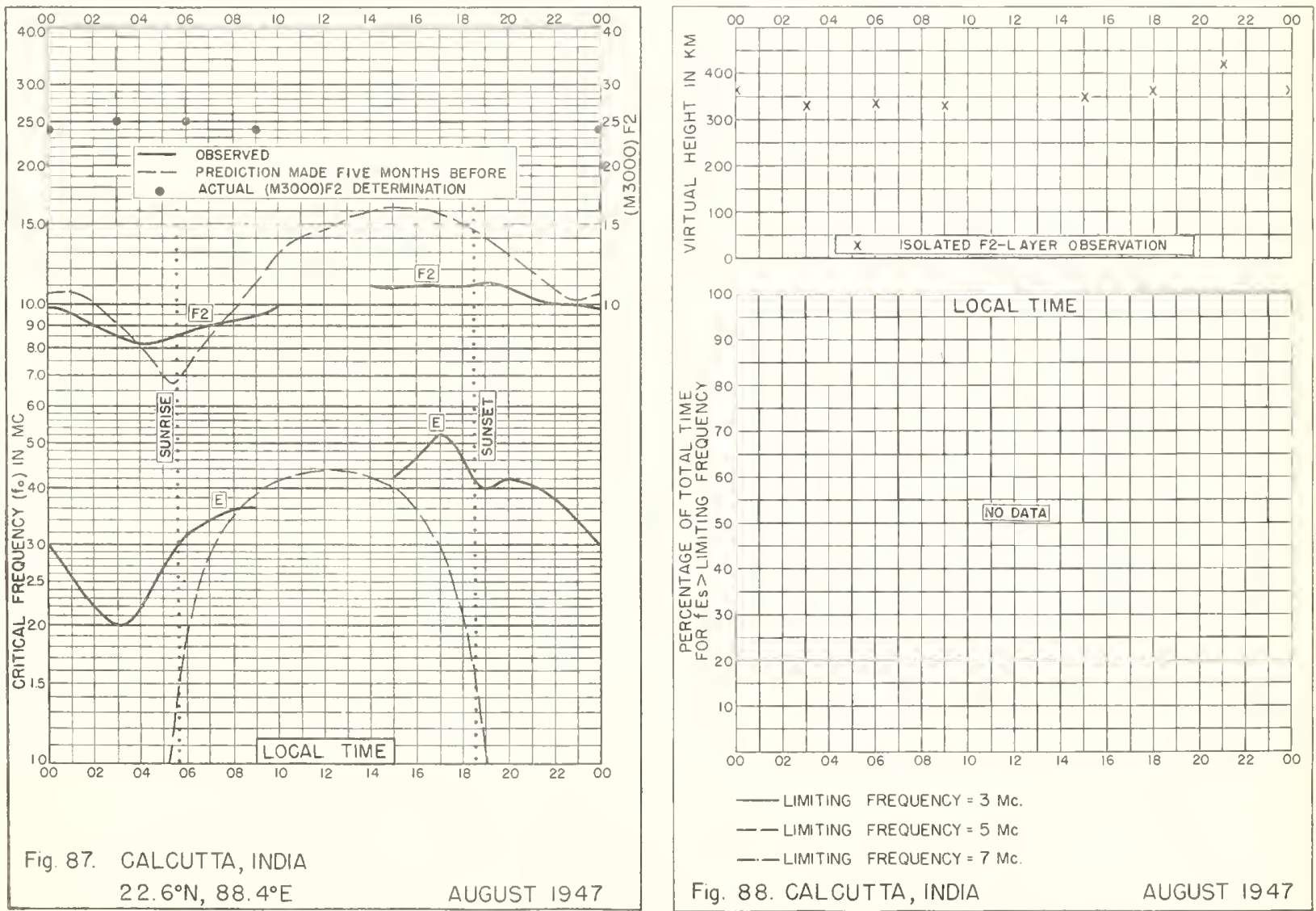


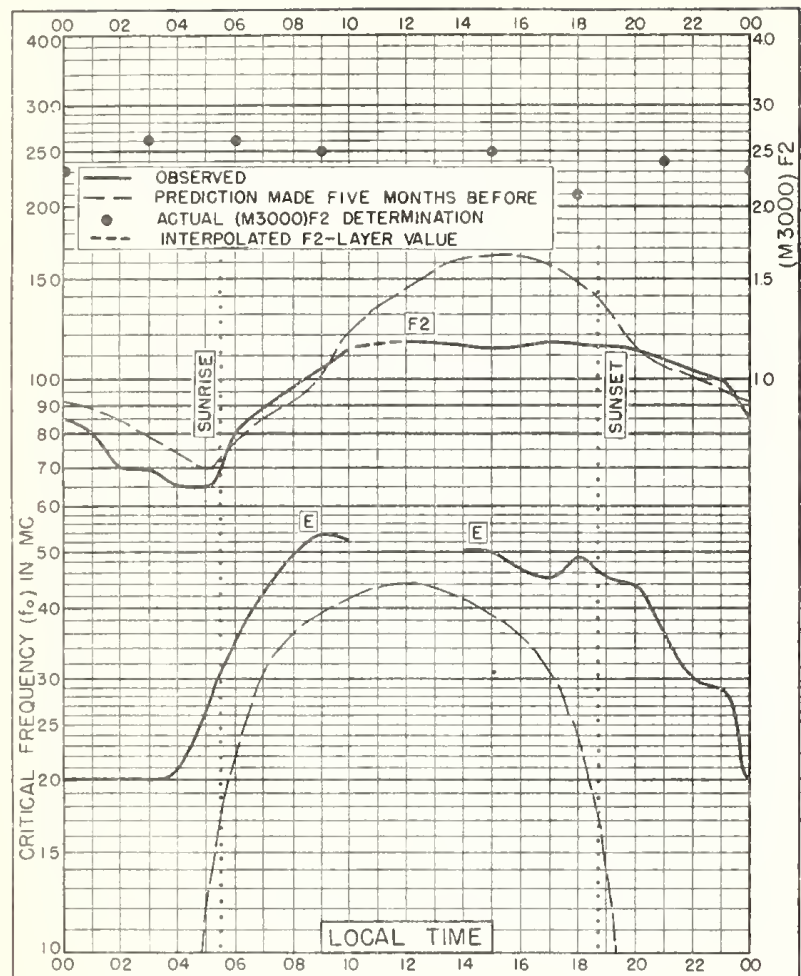

Fig. 89. CALCUTTA, INDIA $22.6^{\circ} \mathrm{N}, 88.4^{\circ} \mathrm{E}$

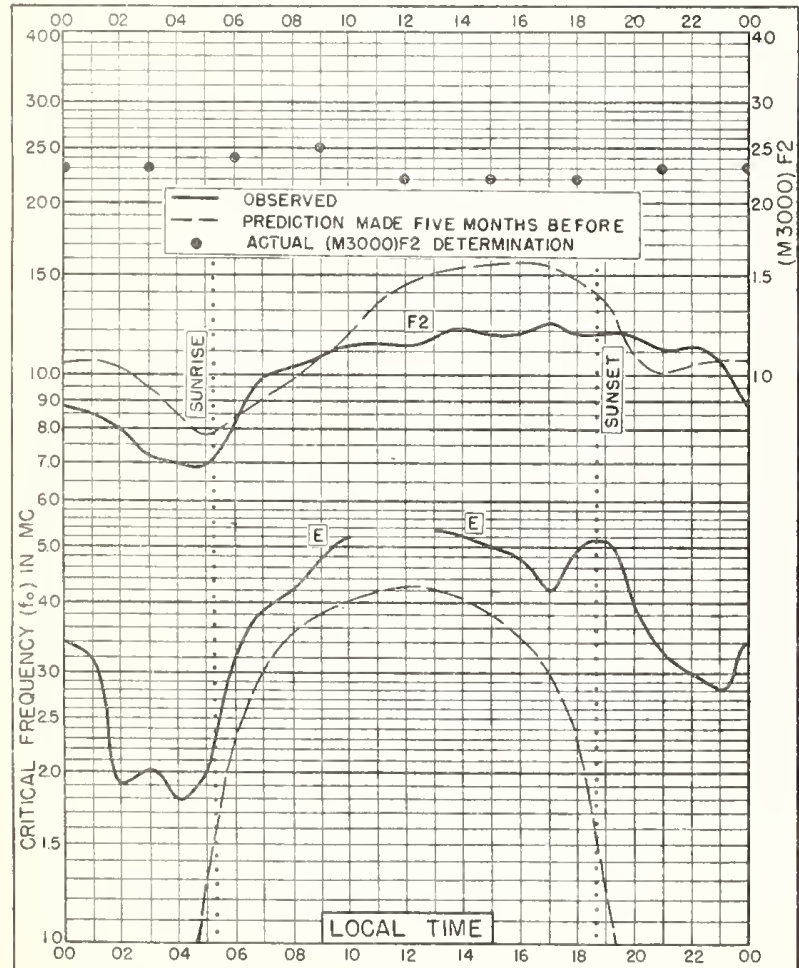

Fig. 91. CALCUTTA, INDIA $226^{\circ} \mathrm{N}, 884^{\circ} \mathrm{E}$ JUNE 1947
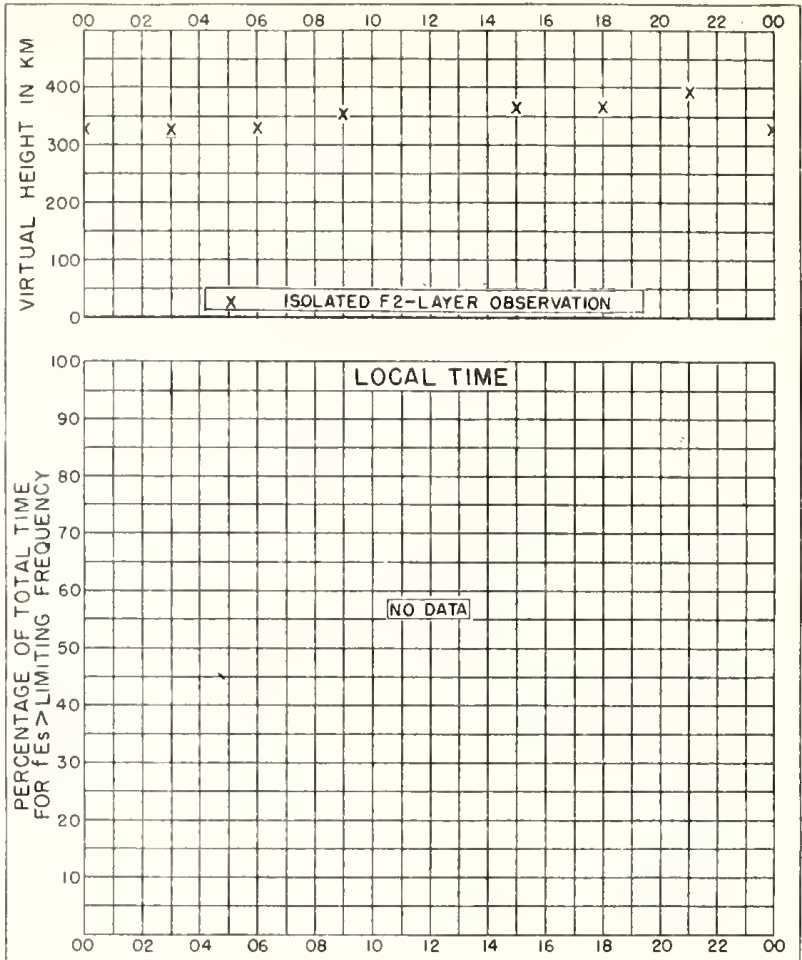

LIMITING FREQUENCY $=3 \mathrm{MC}$

- LIMITING FREOUENCY $=5 \mathrm{MC}$

- - LIMITING FREOUENCY $=7 \mathrm{MC}$.

Fig. 90. CALCUTTA, INDIA

JULY 1947
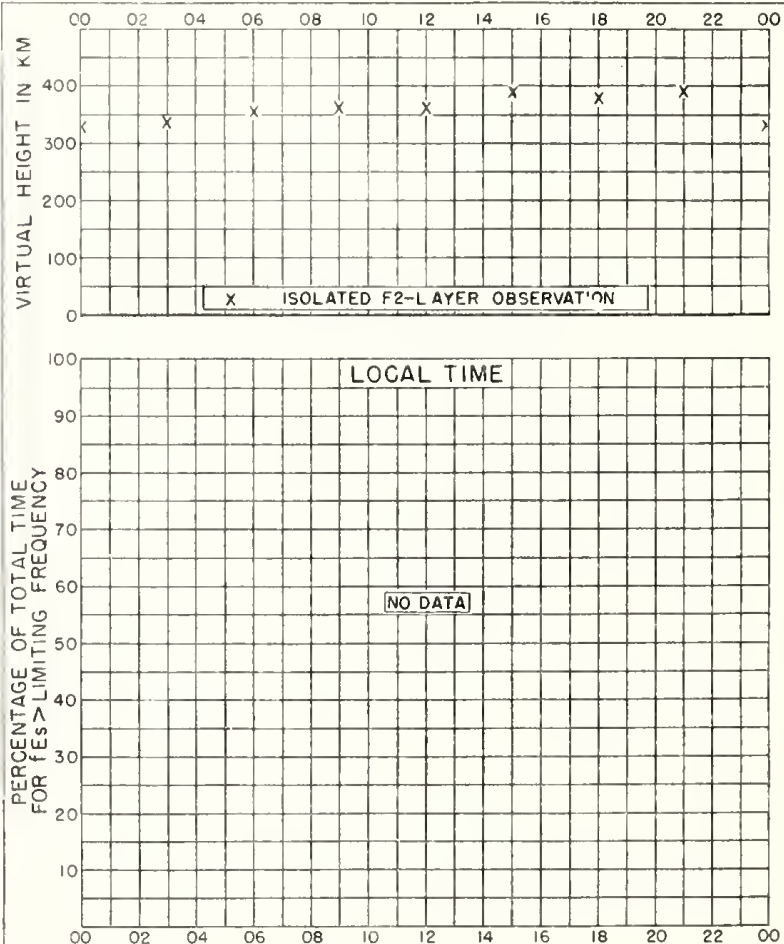

LIMITING FREQUENCY $=3 \mathrm{MC}$.

- LIMITING FREQUENCY $=5 \mathrm{MC}$

- - LIMITING FREQUENCY $=7 \mathrm{MC}$.

Fig. 92. CALCUTTA, INDIA JUNE 1947 


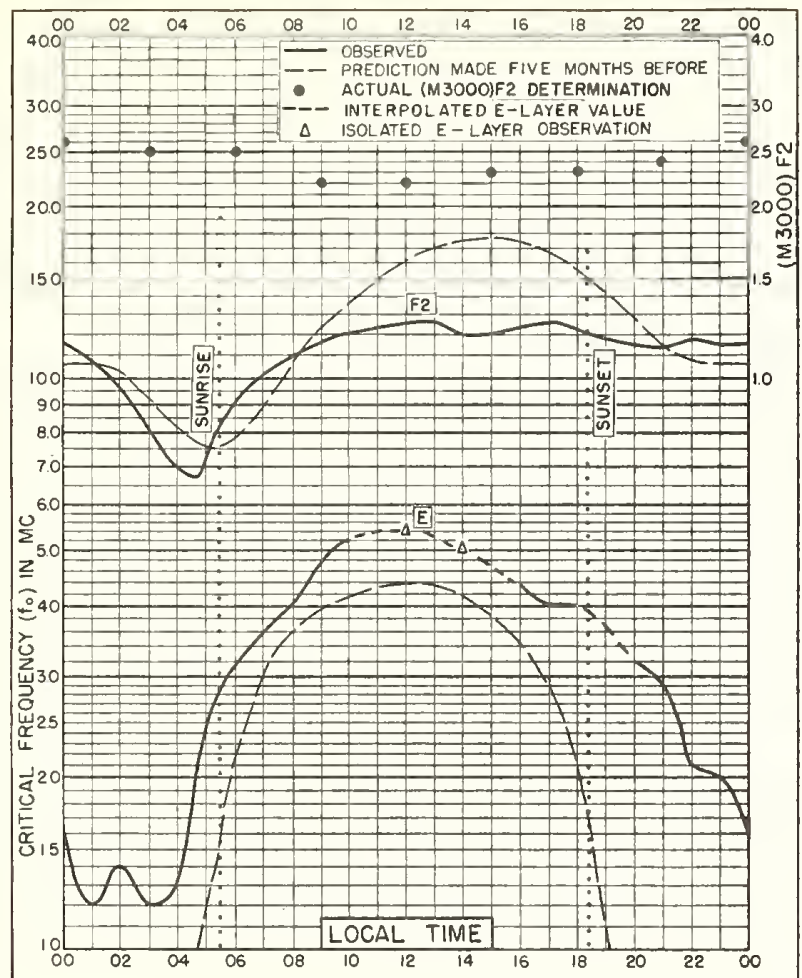

Fig. 93. CALCUTTA, INDIA $22.6^{\circ} \mathrm{N}, 88.4^{\circ} \mathrm{E}$

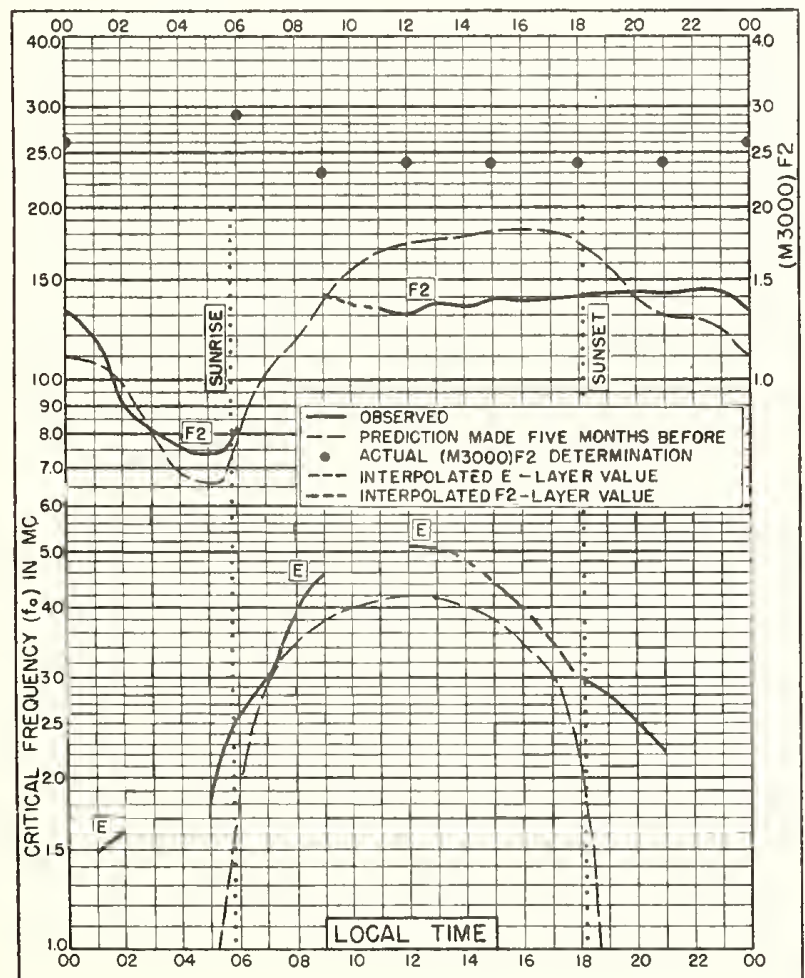

Fig. 95. CALCUTTA, INDIA $22.6^{\circ} \mathrm{N}, 88.4^{\circ} \mathrm{E}$

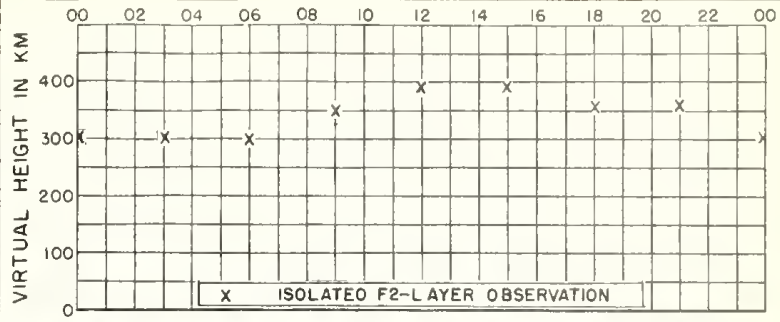

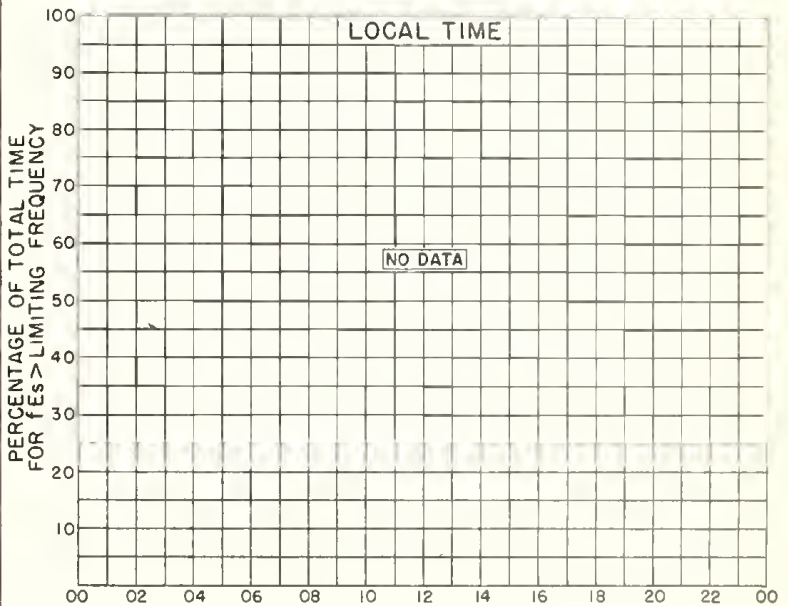

- LIMITING FREQUENCY $=3 \mathrm{MC}$.

- - LIMITING FREQUENCY $=5 \mathrm{MC}$.

-.- LIMITING FREQUENCY $=7 \mathrm{MC}$

Fig. 94. CALCUTTA, INDIA

MAY

1947
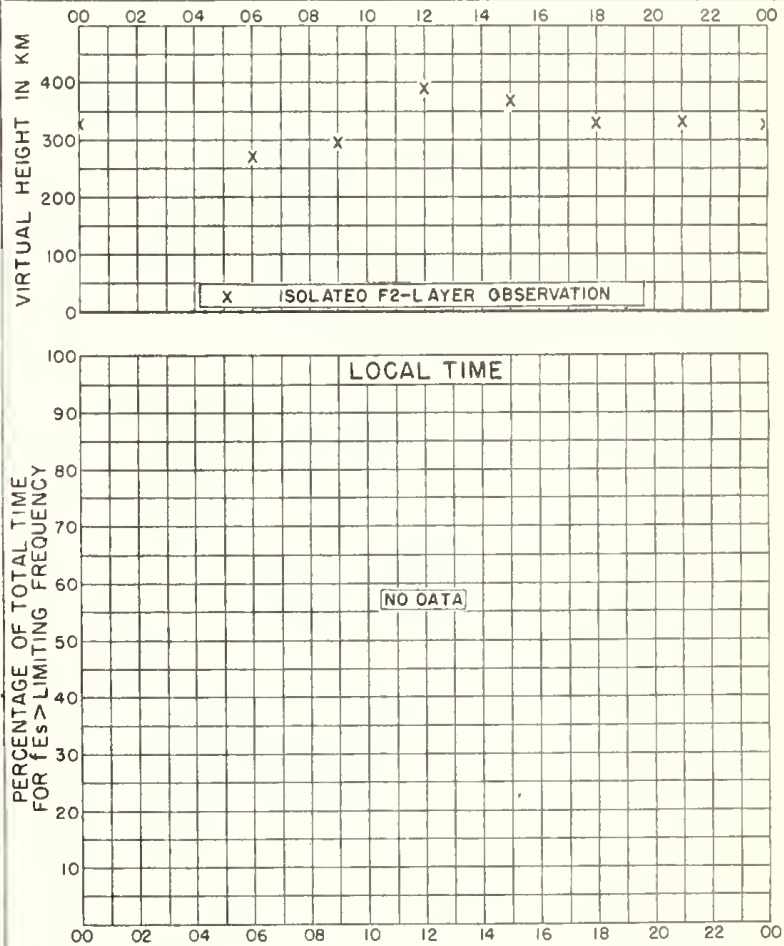

\footnotetext{
- LIMITING FREQUENCY $=3 \mathrm{MC}$.

- LIMITING FREQUENCY $=5 \mathrm{MC}$

-.-LIMITING FREQUENCY $=7 \mathrm{MC}$
}

Fig. 96. CALCUTTA, INDIA 
66
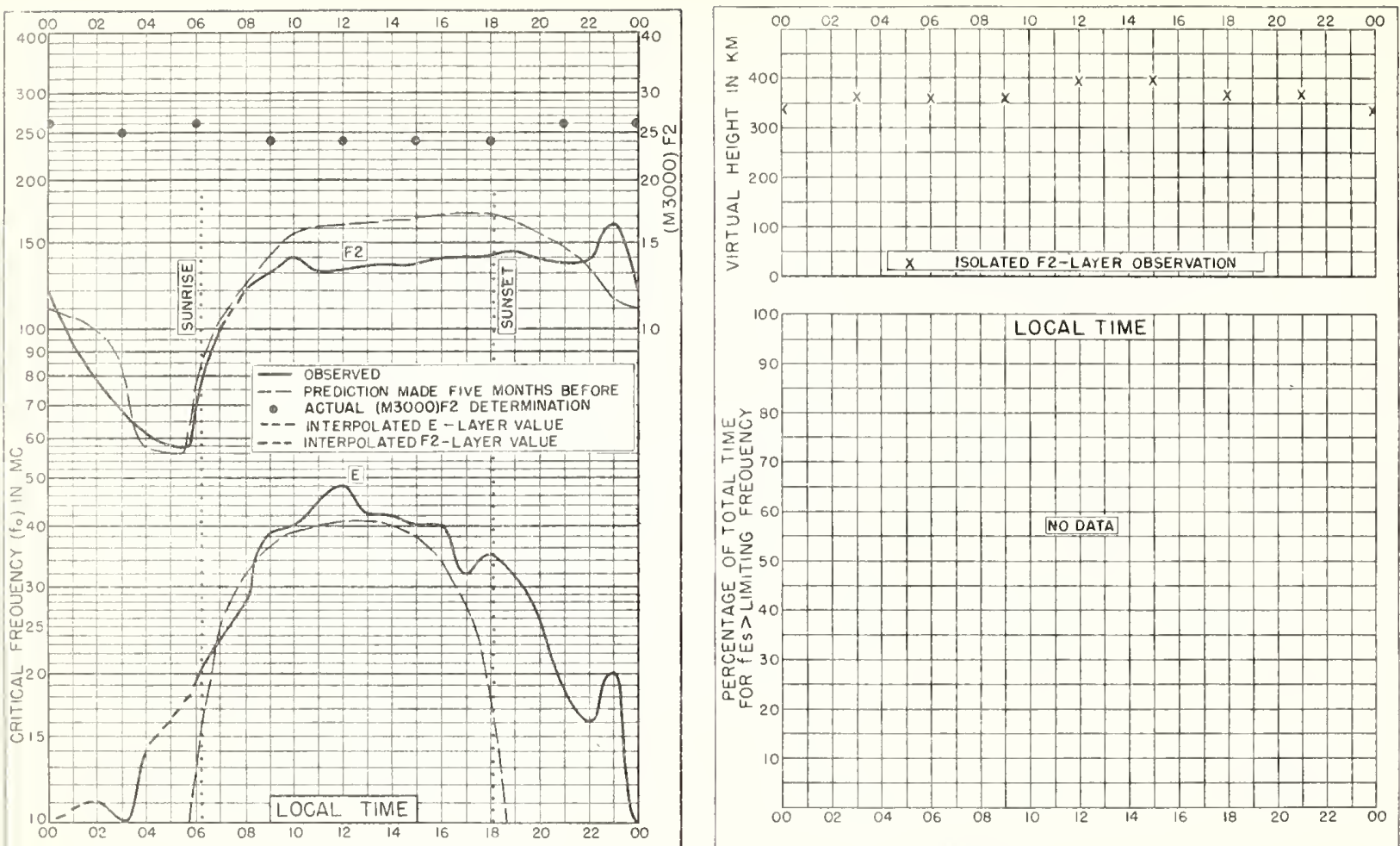

Fig. 97. CALCUTTA, INDIA 22. $6^{\circ} \mathrm{N}, 88.4^{\circ} \mathrm{E}$ MARCH 1947

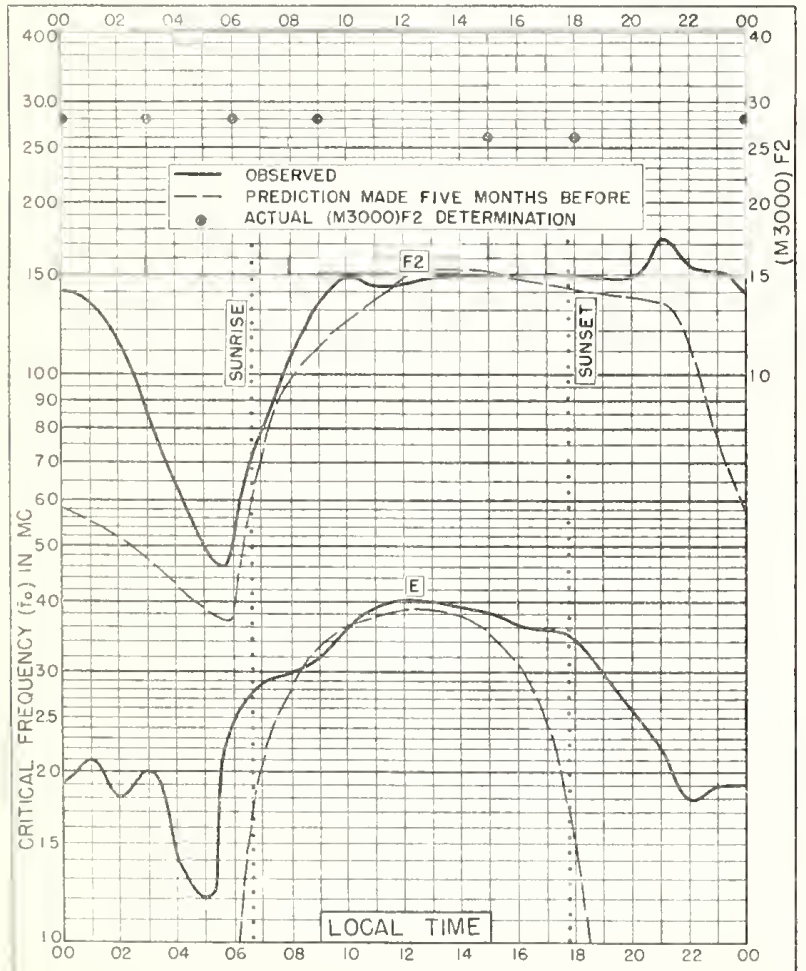

Fig. 99. CALCUTTA, INDIA 22. $6^{\circ} \mathrm{N}, 88.4^{\circ} \mathrm{E}$ FEBRUARY 1947

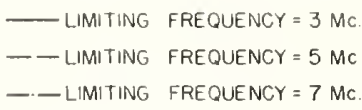

Fig. 98. CAL CUTTA, INDIA

MARCH 1947
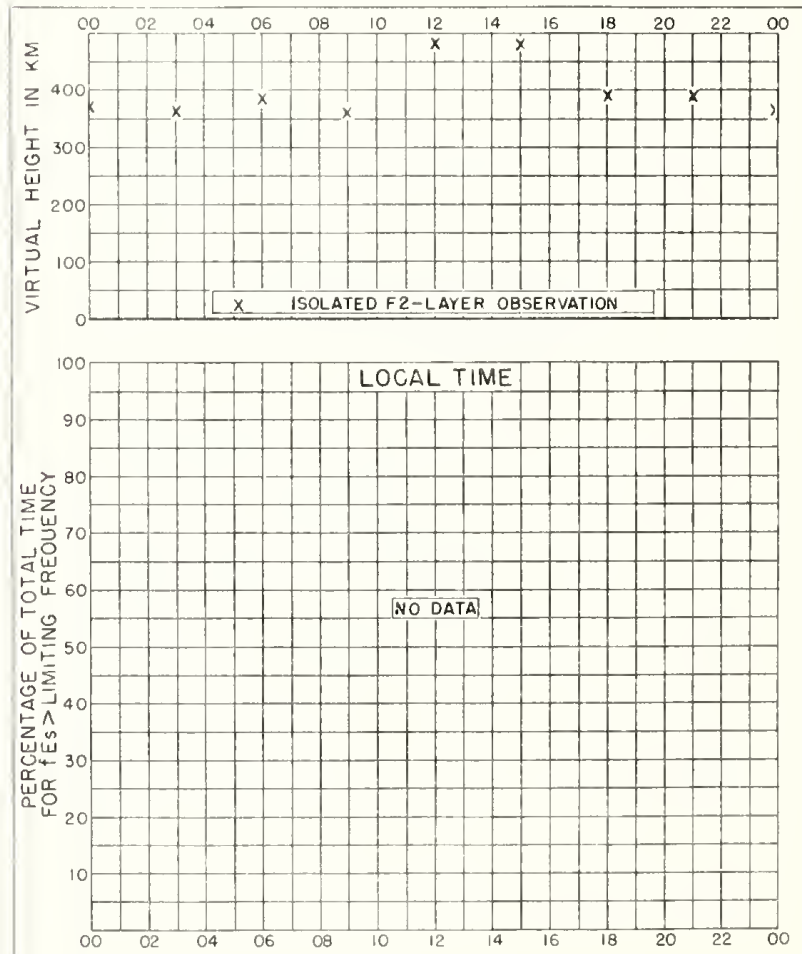

LIMITING FREQUENCY $=3 \mathrm{MC}$

- LIMITING FREQUENCY $=5 \mathrm{MC}$

- - LIMITING FREQUENCY $=7 \mathrm{MC}$

Fig. 100. CAL CUTTA, INDIA

FEBRUARY 1947 


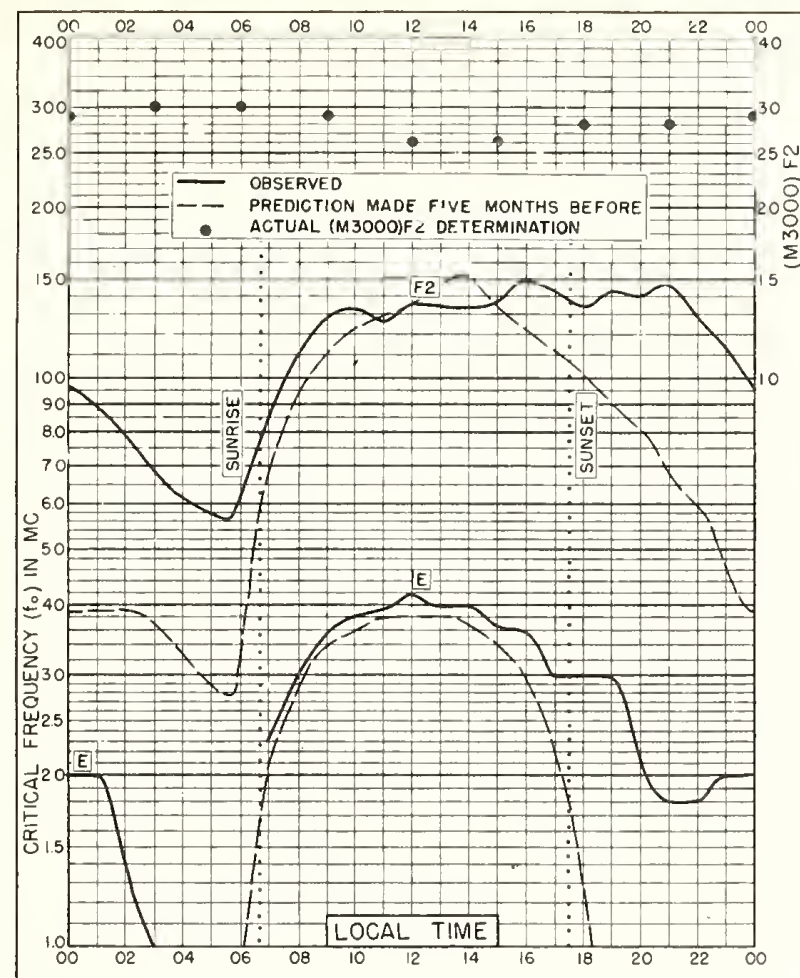

Fig. 101. CALCUTTA, INDIA

22. $6^{\circ} \mathrm{N}, 88.4^{\circ} \mathrm{E}$

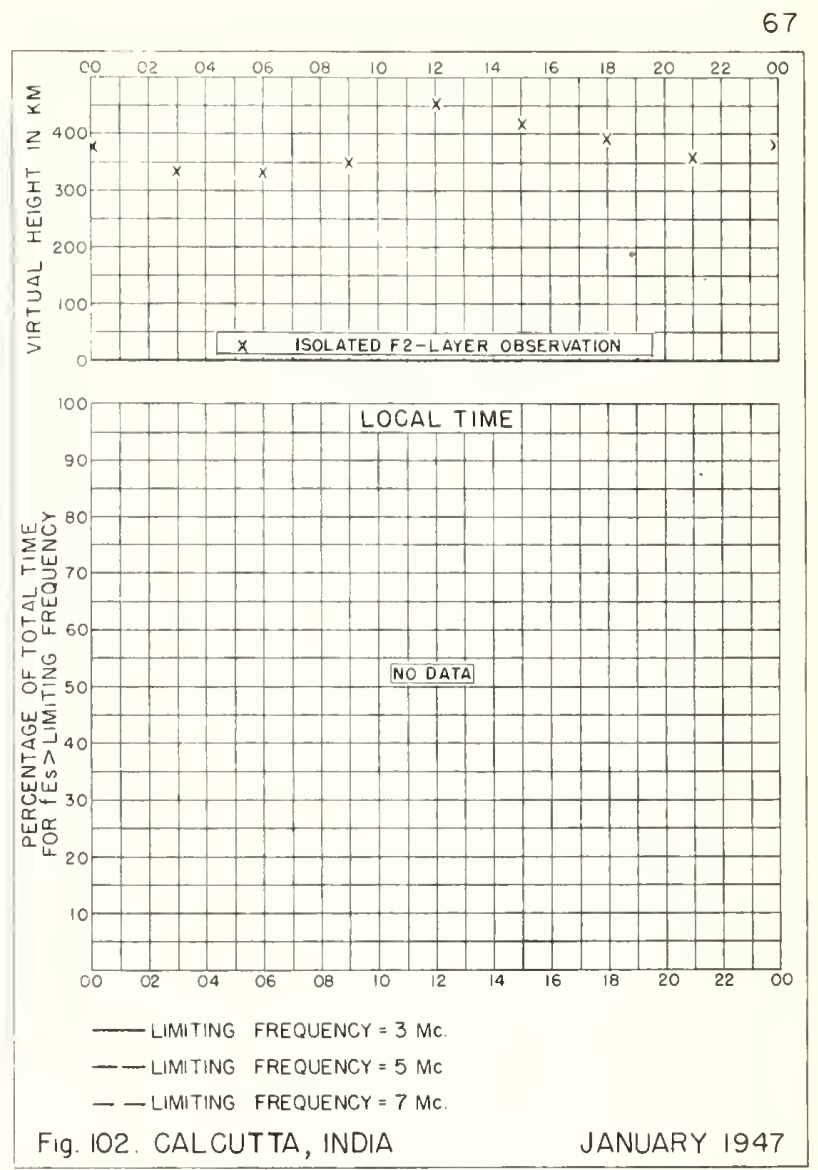

JANUARY 1947 


\section{Index of Tables and Granhs of Ionospheric Data}

\section{in CRPL-F62}

Table page Figure page

Batura Rouge, Louisiana

fugust 1949............... 10

Borabay, India

May 1949 ............... 14

Boston, Missachusetts

August 1949 ............. 10

Calcutta, India

April 1949 .............. 15

March 1949 ............. 15

August 1948 ............. 15

July 1948 ................ 15

Tune 1948 ............... 15

Mау 1948 . . . . . . . . . . . 15

April 1948 ..., ........... 16

March 1948 ............. 16

60

February 1948 .............. 16

61

January 1948 .............. 16

61

December 1947 . . . . . . . . . . 16

62

November 1947 . . . . . . . 16

September $1947 \ldots \ldots 17$

August 19,47............... 17

Ji21y 1947 ................ 17

Jure 1947 ..................... 17

May 1947 ............... 17

Anril 1947 ............. 17

liarch 1947 .............. 18

66

February 1947 ............. 18

66

January 1947 ............ 18

June $1949 \ldots \ldots 13$

Christchurek, New Zeriand

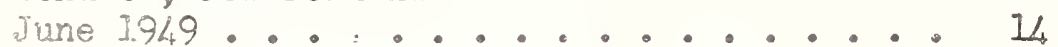

c? ungking, China

June $1949 \ldots \ldots 13$

Delhi, Indis

My $1949 \ldots . . . . . . . . .214$

Tukaura, Japan

Tune $1949 \ldots \ldots 12$

Guam I.

August $1949 \ldots \ldots$. . . . . . . II

August 1949............ II 
Index (CRPLF62, continued)

Table page Figure page

Lanchow, China

May $1949 \ldots \ldots 1_{4}$

54

Lindau/Harz, Germany

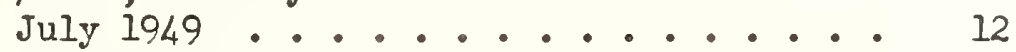

June 1949 ................. 12

Madras, India

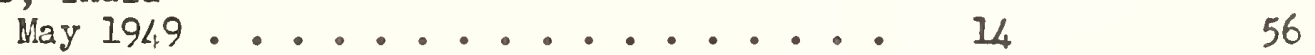

Maui, Hawaii

August 1949 ............... 11

Oslo, Norway

August $1949 \ldots \ldots 10 \quad \ldots 2$

Palmyra I. August 1949 . . . . . . . . 11

San Francisco, California Auqust 1949 ............ 10

San Juan, Puer to Rico August $1949 \ldots \ldots 11$

Shibata, Japan June 1949 ............... 13

Tiruchirapalli, India

Tokyo, Japan June 1949 .............. 13

Trinidad, British West Indies

iTakkanai, Japan June 1949 .............. 12

50

Washington, D. C. September $1949 \ldots \ldots 10$

42

Watheroo, W. Australia June $1949 \ldots \ldots 13$

White Sands, New Mexico August 1949 . . . . . . . 10

44

Yamakawa, Japan June 1949 


\section{CRPL and IRPL Reports}

[A list of CRPL Section Reports is available from the Central Radio Propagation Laboratory upon request] Daily:

Radio disturbance warnings, every half hour from broadcast station WWV of the National Bureau of Standards.

Telephoned and telegraphed reports of ionospheric, solar, geomagnetic, and rario propagation data.

Weekly:

CRPL-J. Radio Propagation Forecast (of days most likely to be disturbed during following month).

Semimonthly:

CRPL-Ja. Semimonthly Frequency Revision Factors for CRPL Basic Radio Propagation Prediction Reports.

Monthly:

CRPL-D. Basic Radio Propagation Predictions-Three months in advance. (Dept. of the Army, TB 11-499-, monthly supplements to TM 11-499; Dept. of the Navy, DNC-13-1 ( ), monthly supplements to DNC-13-1.)

CRPL-F. Ionospheric Data.

Quarterly:

*IRPL-A. Recommended Frequency Bands for Ships and Aircraft in the Atlantic and Pacific.

*IRPL-H. Frequency Guide for Operating Personnel.

Circulars of the National Bureau of Standards:

NBS Circular 462. Ionospheric Radio Propagation.

NBS Circular 465. Instructions for the Use of Basic Radio Propagation Predictions.

Reports issued in past:

IRPL-C61. Report of the International Radio Propagation Conference, 17 April to 5 May 1944.

IRPL-G1 through G12. Correlation of D. F. Errors With Ionospheric Conditions.

IRPI-R. Nonscheduled reports:

R4. Methods Used by IRPL for the Prediction of Ionosphere Characteristics and Maximum Usable Frequencies.

R5. Criteria for Ionospheric Storminess.

R6. Experimental Studies of Ionospheric Propagation as Applied to the Loran System.

R7. Second Report on Experimental Studies of Ionospheric Propagation as Applied to the Loran System.

R9. An Automatic Instantaneous Indicator of Skip Distance and MUF.

R10. A Proposal for the Use of Rockets for the Study of the Ionosphere.

Ri1. A Nomographic Method for Both Prediction and Observation Correlation of Ionosphere Characteristics.

R12. Short Time Variations in Ionospheric Characteristics.

R14. A Graphical Method for Calculating Ground Reflection Coefficients.

R15. Predicted Limits for F2-layer Radio Transmission Throughout the Solar Cycle.

R17. Japanese Ionospheric Data-1943.

R18. Comparison of Geomagnetic Records and North Atlantic Radio Propagation Quality Figures-October 1943 Through May 1945.

R21. Notes on the Preparation of Skip-Distance and MUF Charts for Use by Direction-Finder Stations. (For distances out to $4000 \mathrm{~km}$.)

R23. Solar-Cycle Data for Correlation with Radio Propagation Phenomena.

R24. Relations Between Band Width, Pulse Shape and Usefulness of Pulses in the Loran System.

R25. The Prediction of Solar Activity as a Basis for the Prediction of Radio Propagation Phenomena.

R26. The Ionosphere as a Measure of Solar Activity.

R27. Relationships Between Radio Propagation Disturbance and Central Meridian Passage of Sunspots Grouped by Distance From Center of Disc.

R30. Disturbance Rating in Values of IRPL Quality-Figure Scale from A. T. \& T. Co. Transmission Disturbance Reports to Replace T. D. Figures as Reported.

R31. North Atlantic Radio Propagation Disturbances, October 1943 Through October 1945.

R33. Ionospheric Data on File at IRPL.

R34. The Interpretation of Recorded Values of $f E s$.

R35. Comparison of Percentage of Total Time of Second-Multiple Es Reflections and That of $f E s$. in Excess of 3 Mc.

IRPL-T. Reports on tropospheric propagation:

T1. Radar operation and weather. (Superseded by JANP 101.)

T2. Radar coverage and weather. (Superseded by JANP 102.)

CATl-T3. Tropospheric Propagation and Radio-Meteorology. (Reissue of Columbia Wave Propagation Group WPG-5.)

"Items bearing this symbol are distributed only by U.S. Navy. They are issued under one cover as the DNC-14 series. 
